\title{
X-ray Absorption Spectroscopy Characterization of Electrochemical Processes in Renewable Energy Storage and Conversion Devices
}

\author{
by Maryam Farmand
}

B.S. in Chemistry, June, 2003, Shairf University of Technology, Tehran, Iran

A Dissertation submitted to

The Faculty of

Columbian College of Arts and Sciences

of The George Washington University

in partial fulfillment of the requirements

for the degree of Doctor of Philosophy

May $19^{\text {th }}, 2013$

Dissertation directed by

David E. Ramaker

Professor Emeritus of Chemistry in Residence

Stuart Licht

Professor of Chemistry

\begin{abstract}
Notice: This Thesis/Dissertation describes work supported by Brookhaven Science Associates, LLC under Contract No. DE-SC0012704 with the U.S. Department of Energy. The United States Government retains a non-exclusive, paid-up, irrevocable, world-wide license to publish or reproduce the published form of this manuscript, or allow others to do so, for United States Government purposes.
\end{abstract}


UMI Number: 3557518

All rights reserved

INFORMATION TO ALL USERS

The quality of this reproduction is dependent upon the quality of the copy submitted.

In the unlikely event that the author did not send a complete manuscript and there are missing pages, these will be noted. Also, if material had to be removed, a note will indicate the deletion.

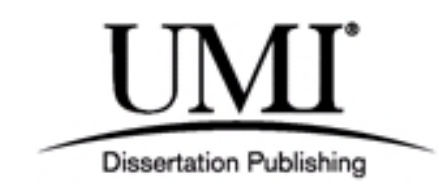

UMI 3557518

Published by ProQuest LLC (2013). Copyright in the Dissertation held by the Author.

Microform Edition () ProQuest LLC.

All rights reserved. This work is protected against unauthorized copying under Title 17, United States Code

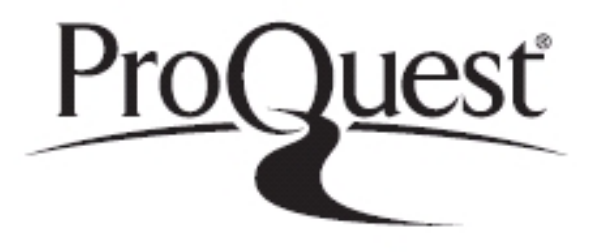

ProQuest LLC.

789 East Eisenhower Parkway

P.O. Box 1346

Ann Arbor, MI 48106 - 1346 
The Columbian College of Arts and Sceinecs of The George Washington University certifies that Maryam Farmand has passed the Final Examination for the degree of Doctor of Philosophy as of March 15th, 2013. This is the final and approved form of the dissertation.

\title{
X-ray Absorption Spectroscopy Characterization of Electrochemical Processes in Renewable Energy Storage and Conversion Devices
}

\author{
Maryam Farmand
}

Dissertaion Research Committee:

David E. Ramaker, Professor Emeritus of Chemistry in Residence, Dissertation Co-Director

Stuart Licht, Professor of Chemistry, Dissertation Co-Director

Akos Vertes, Professor of Chemistry, Committee Member

Hanning Chen, Assistant Professor of Chemistry, Committee Member 
(C) Copyright 2013 by Maryam Farmand All rights reserved 
Dedicated to My Loving Parents and Husband and in Memory of My grandmother 


\section{Acknowledgments}

First and foremost, I would like to express my deep gratitude and appreciation for my adviser, Professor David Ramaker, for giving me an incredible opportunity to work in his group at one of the most difficult times in my academic life. Being Professor Ramaker's student gave me a new outlook and passion for science. Not only did I learn everything scientific I know from him, but I learned to thrive on solving scientific problems, to enjoy and be proud of being a scientist. I thank Professor Ramaker for his continuous support and patience with me, for guiding me when I was frustrated and motivating me when I was ready to give up. Professor Ramaker elevated the quality and accuracy of my work enormously at each step and level of my research including this thesis. I will always be grateful and indebted to him for all I learned from him.

I would also like to thank my co-adviser Professor Stuart Licht. It was a great privilege to be able to work with him on the very exciting super-iron project, as well as in his lab. Working in Professor Licht's lab gave me the opportunity to expand my experimental skills significantly, to be involved in a very exciting research project and to develop a deep understanding of various aspects and challenges of sustainable energy research. I am very grateful for all the support and guidance he provided me through the years and for being able to be a part of his very exciting and cutting edge research group.

I wouldn't have been able to succeed without all the support from the Chemistry Department of George Washington University. Especially, I would like to thank our department chair, Professor Michael King, who showed me how concerned and serious our 
department is for the well-being and success of each and every student. Professor King's support, kindness and guidance was essential in my transition to Professor Ramaker's group and my success in finishing my graduate studies. I am grateful to Professor King for offering me a chance to teach organic chemistry lab and later on hiring me to teach general chemistry lab.

For all the financial support, especially for granting me the Presidential Merit Fellowship, I would like to thank George Washington University. I specifically thank Geri Ripkema and the Office of Graduate Assistantship and Fellowships for their kind support over the years.

I am very thankful to my friends and colleagues Anna Korovina and Keegan Caldwell in the Ramaker research group. It was a delight to work with both of them. I like to especially thank my former colleague Dr. Badri Shyam, for teaching me the basics of conducting research with the synchrotron, for spending so much time helping me during the start of my experimental work at Brookhaven National Lab, and for his friendship and guidance over the years. I am also very grateful to all my friends in Prof. Licht's group, especially former colleagues Dr. Susantah Ghosh and Dr. Dianlu Jiang for teaching me some important aspects on the super-iron project.

It was a very exciting and valuable experience to be able to work at a synchrotron facility. I like to thank the management of NSLS at Brookhaven National Laboratory, who facilitated my receiving a clearance to have access to the facility. Mercy Baez from the NSLS user administration office, was very kind, patient and helpful in keeping my access clearance up-to-date. I also am truly grateful for all the help I received from the beamline scientists, Mr. Michael Sullivan, Dr. Eric Farquhar (X3-B) and Dr. Kumi Pandya (X11-B), 
at NSLS, Brookhaven National Lab. They helped me collect data and operate the beamline with accuracy and ease. It was a great privilege to be able to work in such a scientific environment.

I thank the members of my examining committee, Professors Akos Vertes, Stuart Licht, Hanning Chen and Michael Massiah from George Washington University and Dr. Azzam Mansour from the Naval Surface Warfare Center for their kind assistance and attention to my thesis.

Finally I would like to thank my family. I owe everything I am and all I have to my parents Minoo Rafiei and Reza Farmand. My mom always was and will be my role model in life. She is all that I ever dream to achieve both at the moral and personal level as a great human being and as an extremely successful woman. I am grateful to my father, for supporting me and believing in me throughout my life, for picking me up every time I fell down, for always telling me not to be scared and to trust myself, for giving me my selfconfidence. I wouldn't have finished my degree without the love and support of my husband, my love, my best friend and companion, Khashayar Beigi. Thank you for making me want to be a better person every day, being my solace in life, for being there throughout my graduate school years every day, and helping me in every way possible. I would like to thank my two beautiful sisters Mitra and Aida, I cannot express in words how much I love you. Thank you for enduring all the years I was gone from home, not available to participate in your lives as much as I wanted. I have been away from my country and my family for so many years, but I found a new family here. My uncle, Mr. Hossein Arshadi and cousins (Faranak, Saeed, Fariman and Sanaz Arshadi) are my second family, they welcomed me as a daughter and sister, offered me their home, helped me in every way 
when I needed it and never let me feel homesick. In the end, I would like to thank my grandmother, Mrs. Azra Arshadi, who didn't live long enough to see my success. She raised me, loved me and taught me everything about "life". From her, I learned that the most important thing in life is to love and be loved. She was the most wise, incredible, intelligent, funny, positive person I ever knew. I didn't have a chance to say goodbye to her, but I thank her in my heart every day and will eternally love and remember her. 


\begin{abstract}
Dissertation
X-ray Absorption Spectroscopy Characterization of Electrochemical Processes in Renewable Energy Storage and Conversion Devices
\end{abstract}

The development of better energy conversion and storage devices, such as fuel cells and batteries, is crucial for reduction of our global carbon footprint and improving the quality of the air we breathe. However, both of these technologies face important challenges. The development of lower cost and better electrode materials, which are more durable and allow more control over the electrochemical reactions occurring at the electrode/electrolyte interface, is perhaps most important for meeting these challenges. Hence, full characterization of the electrochemical processes that occur at the electrodes is vital for intelligent design of more energy efficient electrodes.

X-ray absorption spectroscopy (XAS) is a short-range order, element specific technique that can be utilized to probe the processes occurring at operating electrode surfaces, as well for studying the amorphous materials and nano-particles making up the electrodes. It has been increasingly used in recent years to study fuel cell catalysts through application of the $\Delta \mu$ XANES technique, in combination with the more traditional X-ray Absorption Near Edge Structure (XANES) and Extended X-ray Absorption Fine Structure (EXAFS) techniques. The $\Delta \mu$ XANES data analysis technique, previously developed and applied to heterogeneous catalysts and fuel cell electrocatalysts by the GWU group, was extended in this work to provide for the first time space resolved adsorbate coverages on both electrodes of a direct methanol fuel cell. Even more importantly, the $\Delta \mu$ technique was applied for the first time to battery relevant materials, where bulk properties such as the oxidation state and local geometry of a cathode are followed. 
In-situ XAS accompanied by electrochemical techniques (CV and RDE) were utilized to study the poisoning of carbon supported polycrystalline platinum clusters by bromide and iodide in acidic medium $\left(1 \mathrm{M} \mathrm{HClO}_{4}\right)$. This study provided not only increased insight into the comparative poisoning effects of halides on the ORR in a fuel cell, but also new insights into the nature of the $\Delta \mu$ magnitude itself. At $1 \mathrm{mM}$ halide concentration, the $\Delta \mu$ magnitudes reflect the relative halide coverage on the electrode surface and increase in the order of the Pt-X bond strength: $\mathrm{I}^{-}>\mathrm{Br}^{-}>\mathrm{Cl}^{-}$. However, this trend is reversed at higher concentration $(10 \mathrm{mM})$, where the surface is fully covered by halide anion. At this concentration, the $\Delta \mu$ magnitude reflects the Pt-X adlayer coordination number which decreases with increasing anion size. In the case of strongly bonded adsorbates (e.g., O, $\mathrm{OH}, \mathrm{CO}$ and $\mathrm{H}$ ) on a $\mathrm{Pt}$ electrode in a fuel cell, such as that studied in all previous applications of the $\Delta \mu$ technique, the $\Delta \mu$ magnitude provided a reasonable estimate of the relative adsorbate coverage as a function of potential. However, in the case of the large, highly polarizable and weakly bonded anions, such as the halides in this study, this is not the case, especially at lower concentrations of the adsorbate. In this special case, the $\Delta \mu$ magnitudes were not directly proportional to $\mathrm{X}^{-}$coverage, but decreased as the Pt-X bond changed covalent character with potential.

Current variations across the surface of an electrode are known to impact a fuel cell's performance. To study this, in-operando XAS experiments were conducted to follow the spatial distribution of different adsorbates such as $\mathrm{OH}, \mathrm{H}, \mathrm{O}$, and $\mathrm{CO}$ on both the anode and cathode in a direct methanol fuel cell (DMFC). A specially designed in-situ cell was utilized, which had three different X-ray windows at different locations relative to the inlets 
and outlets of the fuel (methanol) and oxidizer $\left(\mathrm{O}_{2}\right)$ feeding the cell. Fluorescence and transmission data were collected at both the cathodic and anodic sides. The results revealed a very strong "cross-talk" between the anode and cathode, i.e. a strong correlation between the adsorbate coverages on the two sides. At higher oxygen flow rates, it is the anode that dictates the $\mathrm{OH}$ coverage on the cathode, and at lower oxygen flow rate, this "cross-talk" effect is reversed with the cathode dictating the methanol oxidation rate on the anode; i.e., the local adsorbate coverage on both electrodes is determined by the electrode with the slowest reaction rate. These results directly highlight the importance of the local $\mathrm{H}^{+}$concentration for the oxygen reduction at the cathode and under $\mathrm{O}_{2}$ starvation conditions reveal the nature of the adsorbates on both the anode and cathode, when the DMFC is operating in galvanic mode at low cell potential and in electrolytic mode at high cell potential. .

Hexavalent iron salts release three electrons upon reduction and can offer intrinsic capacities as high as $601 \mathrm{mAh} / \mathrm{g}$. However, their discharge product suffers from poor conductivity and low reversibility. The particle size effect on the electrochemical performance of $\mathrm{K}_{2} \mathrm{FeO}_{4}$ was studied utilizing ex-situ XAS measurements. A low heat, gentle and mechano-synthesized pathway was employed to produce nano-sized $\mathrm{K}_{2} \mathrm{FeO}_{4}$ particles. SEM results showed that the particle size was reduced from around 30 microns to about 80-100 nanometers by this ball mill procedure. The final discharge product was identified as primarily $\gamma-\mathrm{Fe}_{2} \mathrm{O}_{3}$. The $\Delta \mu$ analysis was conducted both on the Fe pre-edge that originates from $1 \mathrm{~s}$ to $3 \mathrm{~d}$ transitions and on the Fe K-edge at $7112.0 \mathrm{eV}$ that reflects to 1s to np transitions. The K-edge $\Delta \mu$ amplitudes follow the Fe oxidation state change, while the pre-edge $\Delta \mu$ amplitudes reflect the local geometry change of the hexavalent $\mathrm{Fe}$ salt 
from octahedral to tetrahedral. Linear combination fitting analysis of the EXAFS region to undischarged $\mathrm{K}_{2} \mathrm{FeO}_{4}$ and $\gamma-\mathrm{Fe}_{2} \mathrm{O}_{3}$ standards was utilized to follow the structure. The nano-particles do not show any structural change up to nearly $55 \%$ of the discharge, while structural changes for micron-sized particles start as low as $15 \%$ of the discharge.

Finally $x$-situ XAS measurements were also conducted on micron-sized $\mathrm{BaFeO}_{4}$ and compared with the results on the $\mathrm{K}_{2} \mathrm{FeO}_{4}$. Both nano- $\mathrm{K}_{2} \mathrm{FeO}_{4}$ and $\mathrm{BaFeO}_{4}$ exhibit higher experimental capacities and superior electrochemical performance compared to micron size $\mathrm{K}_{2} \mathrm{FeO}_{4}$. The mechanism by which the $\mathrm{BaFeO}_{4}$ and nano- $\mathrm{K}_{2} \mathrm{FeO}_{4}$ materials hold their structures stable are quite different. During the discharge process in these alkaline batteries, $\mathrm{H}_{2} \mathrm{O}$ intercalates into the cathode lattice through the electrolyte and membrane separator, while electrons travel from the anode to the cathode through the external circuit. Nanoparticles are able to relieve the stress induced by the oxidation through intercalation of water. This is possible because of the shorter diffusion lengths and the increased electrode/electrolyte surface in the nanoparticle. This water intercalation enables the Fe to maintain its original local geometry, and the lattice expansion caused by this intercalation is better accommodated in the micron-sized particles. In the case of $\mathrm{BaFeO}_{4}, \mathrm{XRD}$ and EXAFS results show that the one dimensional intercalation channels are expanded and more symmetrical compared to that of the micron sized $\mathrm{K}_{2} \mathrm{FeO}_{4}$ indicating that $\mathrm{BaFeO}_{4}$ starts with an already expanded lattice and thus intercalation of $\mathrm{H}^{+}$to the cathode is facilitated. This is also the reason behind the higher charge transfer rates in $\mathrm{BaFeO}_{4}$. Further, stronger cation-anion interactions in $\mathrm{BaFeO}_{4}$ also delays the phase separation of the discharge products, $\mathrm{Ba}(\mathrm{OH})_{2}$ and $\gamma-\mathrm{Fe}_{2} \mathrm{O}_{3}$. All of these factors contribute to higher structural stability observed in $\mathrm{BaFeO}_{4}$, increasing its reversibility for more xii 
charge/discharge cycles. In summary, to increase the reversibility of super-iron salts, either the particle size should be reduced to the nano domain and/or a salt with higher anioncation interaction and initial expanded lattice should be utilized. 


\section{Table of Contents}

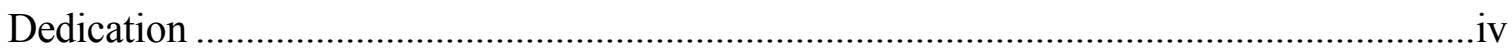

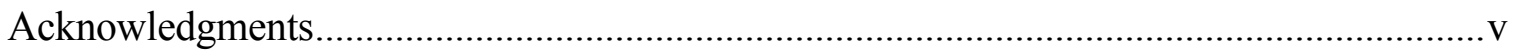

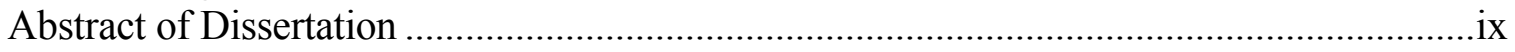

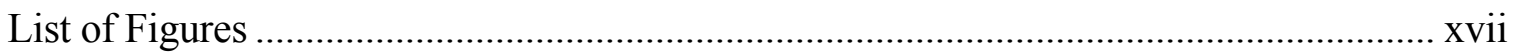

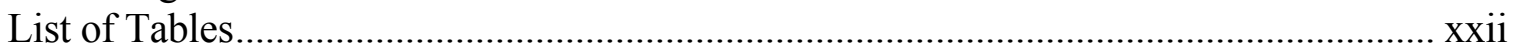

Glossary of Terms ................................................................................................. Xxiii

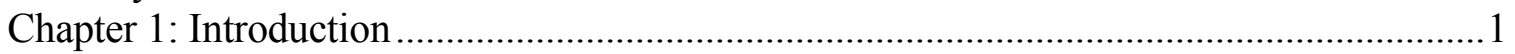

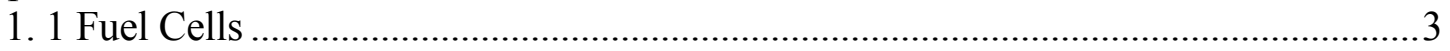

1.1.1 Basic Operation Principles of Fuel Cells ....................................................... 6

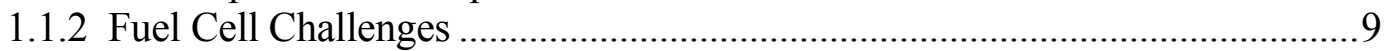

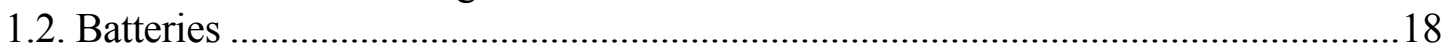

1.2.1. Novel Cathode Materials for High Capacity Batteries .................................20

1.2.2. Classes of Cathode Materials ....................................................................21

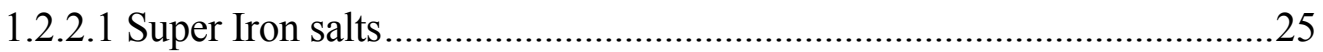

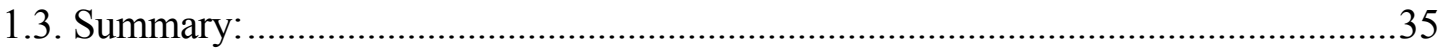

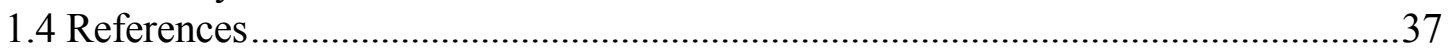

Chapter 2: Introduction to X-ray Absorption Spectroscopy: Experimental and

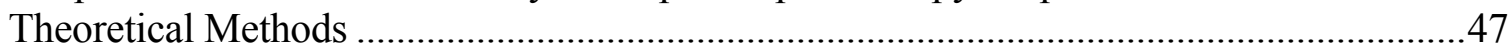

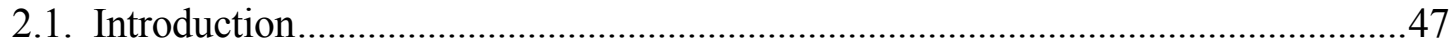

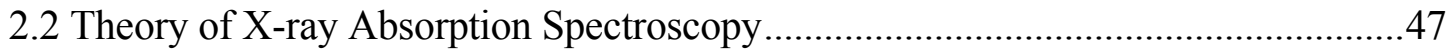

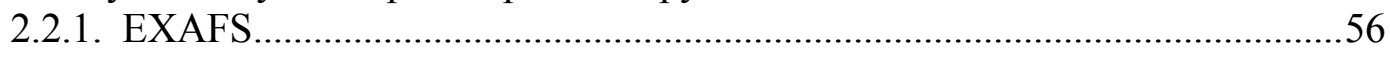

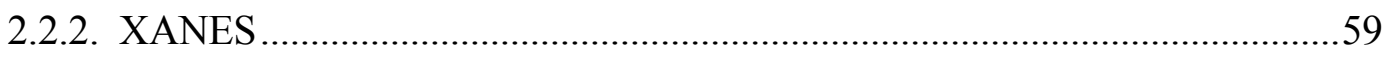

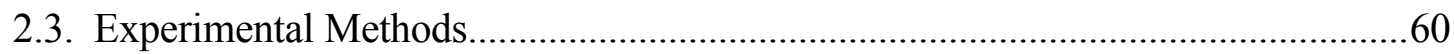

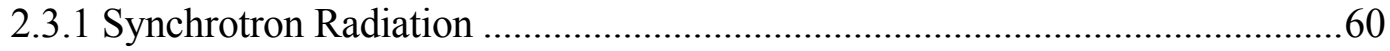

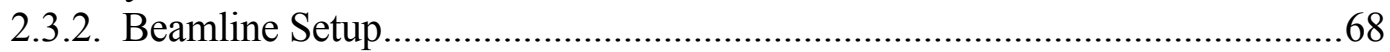

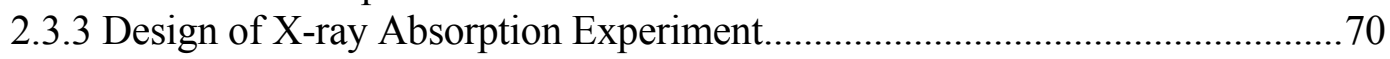

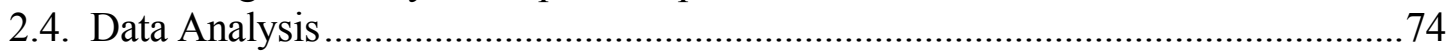

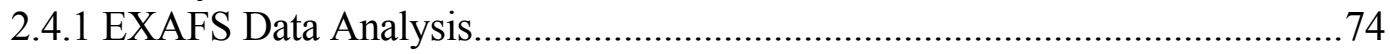

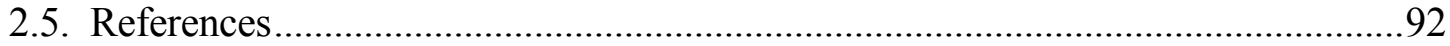

Chapter 3: In-Situ X-ray Absorption Spectroscopy Studies on Adsorption of Bromide

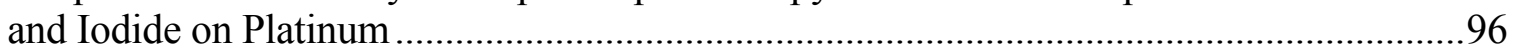

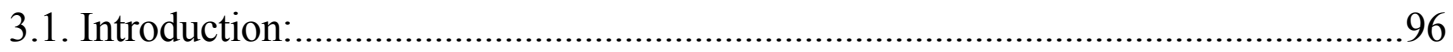

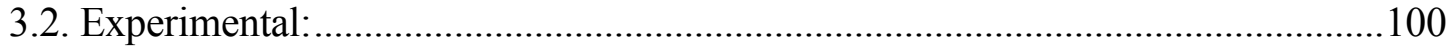

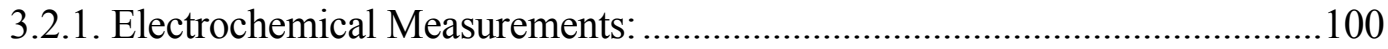

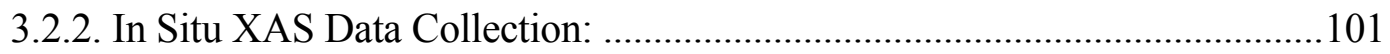

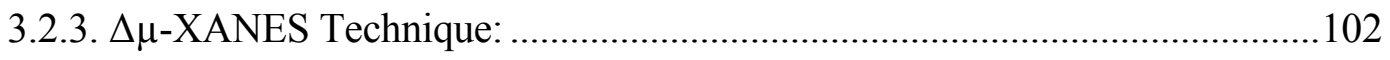

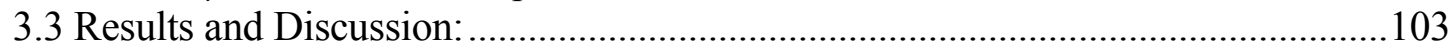

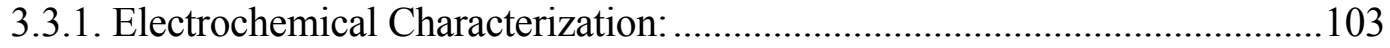

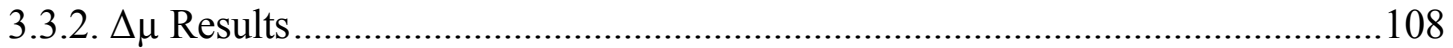

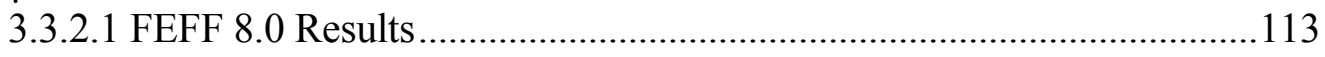

3.3.2.2. Halide Adsorption and Rearrangement ...........................................113

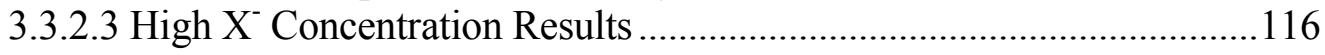

3.3.2.4. Low Concentration Results............................................................118 
3.4. Summary and Conclusions:.................................................................................. 125

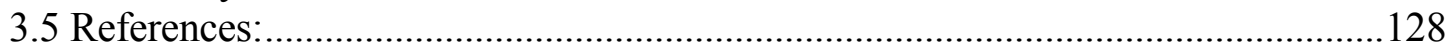

Chapter 4: Space Resolved, In Operando X-ray Absorption Spectroscopy:

Investigations on both the Anode and Cathode in a DMF.................................................132

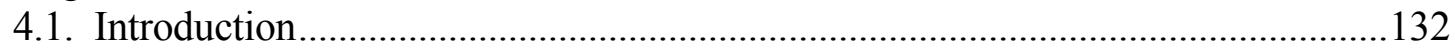

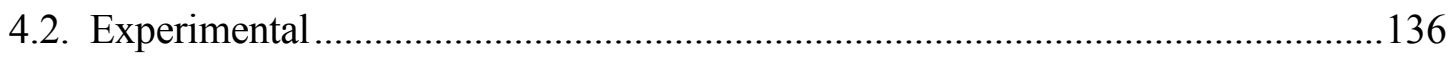

4.2.1 Membrane electrode assembly preparation .................................................136

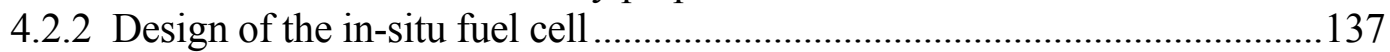

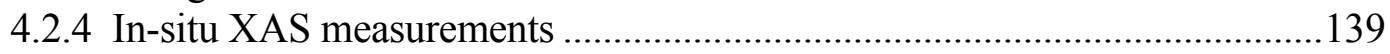

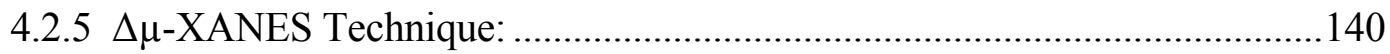

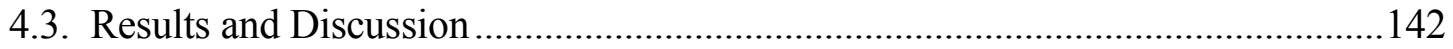

4.3.1 Estimation of anode and cathode potential................................................142

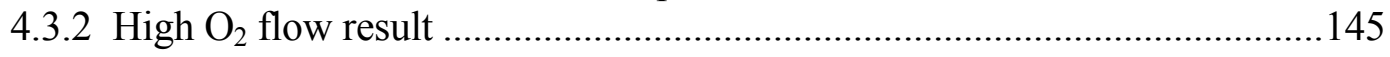

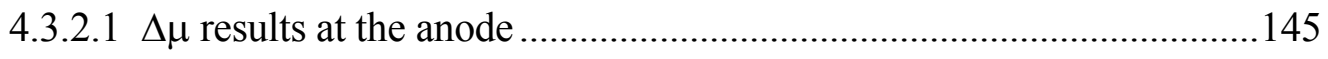

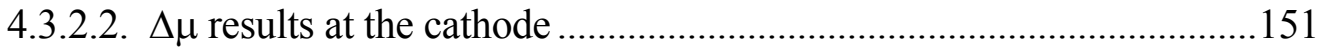

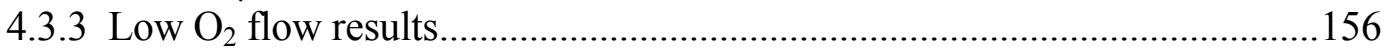

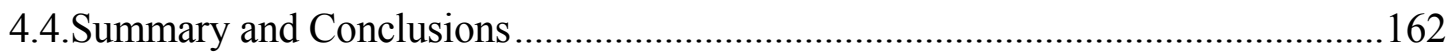

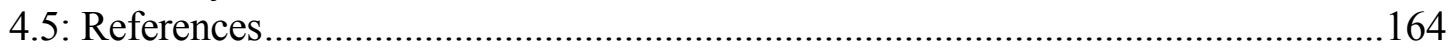

Chapter 5: Super-iron Nanoparticles with Facile Cathodic Charge Transfer ......................168

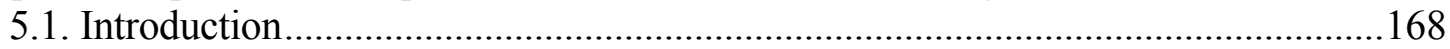

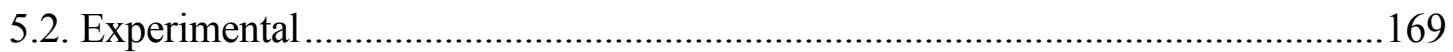

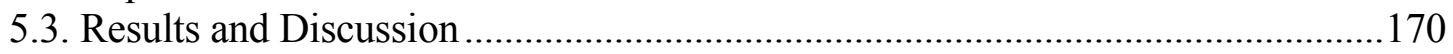

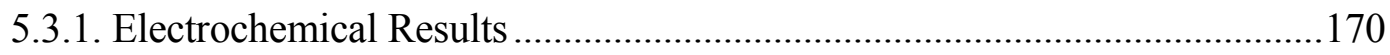

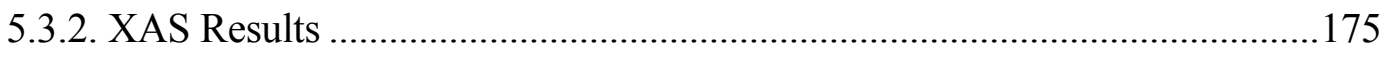

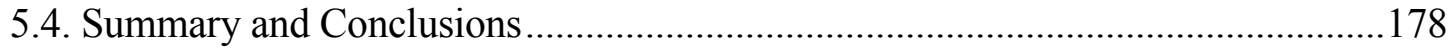

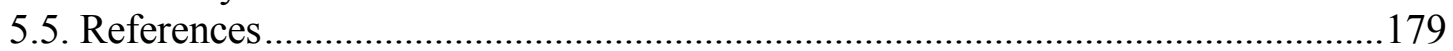

Chapter 6: Studying the Reversibility of Multi-electron Charge Transfer in Fe(VI)

Cathodes Utilizing X-ray Absorption Spectroscopy..........................................................182

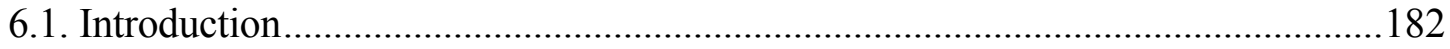

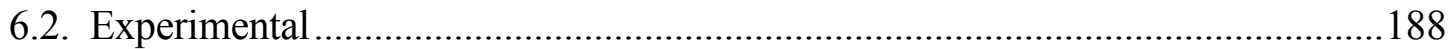

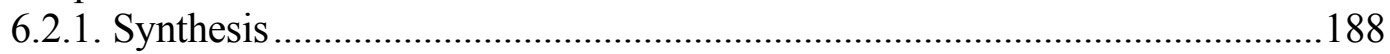

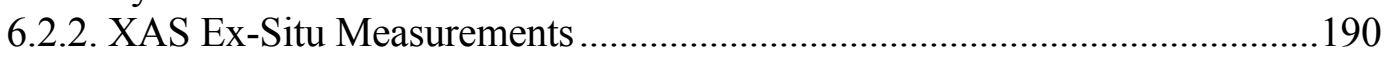

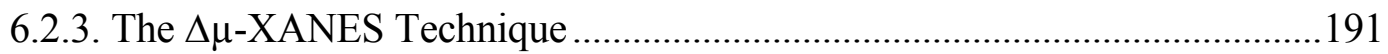

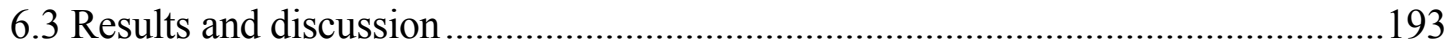

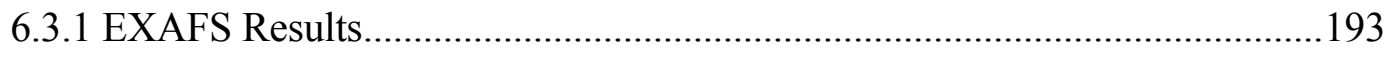

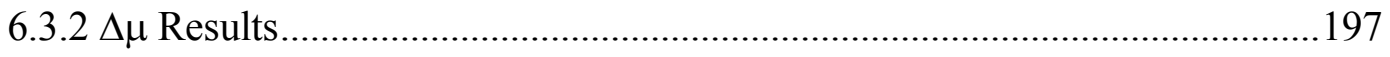

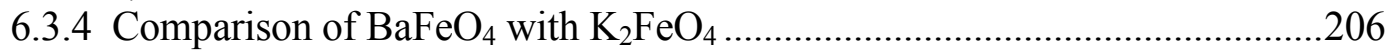

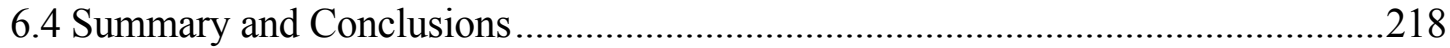

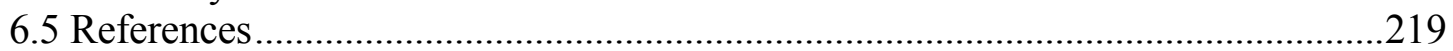

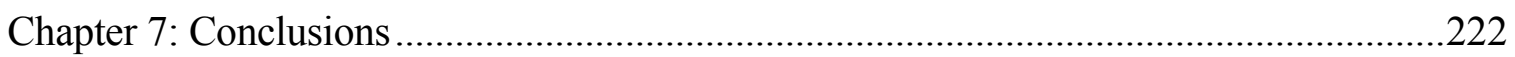

7.1. Adsorption of bromide and iodide on platinum......................................................222

7.2. Space-resolved, in Operando XAS investigations in a DMFC ..............................223

7.3. Super-iron nanoparticles with facile cathodic charge transfer .................................225 
7.4 Studying the Reversibility of Multi-electron Charge Transfer in Fe(VI)

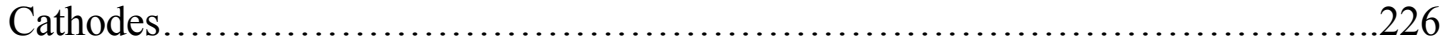

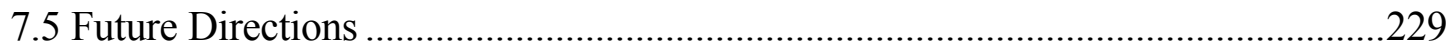

7.6. References: 


\section{List of Figures}

Figure1.1 Projection of greenhouse gas pollution levels in U.S. over the 21st century by light duty vehicles only. Figure adapted from a study by C.E.S. Thomas.

Figure 1.2 Schematic of a Proton Exchange Membrane (PEM) fuel cell Adapted from

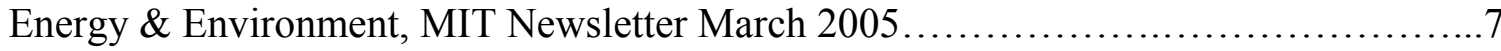

Figure 1.3 A simplified schematic of the ORR pathway adapted from works by Paulus et. al. and Wroblowa et. al............................................................

Figure 2.1. X-ray energies for the $\mathrm{K}$ - and LIII- edges for various elements as a function of their atomic number.

Figure 2.2 Schematic of X-ray absorption processes: a) Excitation of a core-level and ejection of a photoelectron, $b$ ) backscattering by neighboring atoms resulting from the ejection of the photoelectron.

Figure 2.3. Schematic of different backscattering events occurring in an absorption

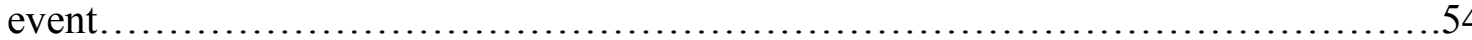

Figure 2.4. Schematic of The National Synchrotron Light Source at Brookhaven National Laboratory, Upton, N.Y. Courtesy of NSLS, Brookhaven National Laboratory (www.bnl.gov)

Figure 2.5 Experimental setup at the beamline for transmission and fluorescence measurement modes

Figure 2.6 Schematic of a double crystal monochromator utilized in tuning the beam energy...... 
Figure 2.7. X3-B beamline setup at NSLS, when running an in-situ XAS experiment

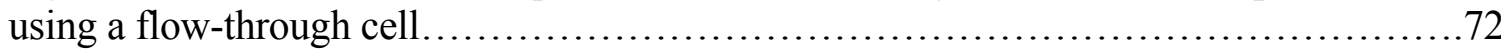

Figure 2.8. Schematic of data analysis procedures for both the XANES and EXAFS techniques

Figure 2.9. Adsorption sites commonly observed for small adsorbates

Fiure 2.10. Re-distribution of electronic charge on atoms in a cluster induced by adsorbate.The presence of adsorbates effectively reduces the distribution of electron density between the Pt atoms.

Figure 3.1. ORR polarization curves for $30 \mathrm{wt} . \% \mathrm{Pt} / \mathrm{C}$ the indicated concentrations of $\mathrm{KBr}$ in $1 \mathrm{M} \mathrm{HClO}_{4}$ taken at $900 \mathrm{RPM}$ at $20^{\circ} \mathrm{C}$. In each case the electrolyte was saturated with $\mathrm{O}_{2}$

Figure 3.2 Cyclic voltammograms of $30 \mathrm{wt} . \% \mathrm{Pt} / \mathrm{C}$ collected using the indicated concentrations of a) $\mathrm{KBr}$ and b) $\mathrm{KI}$. The scans were collected at $20 \quad \mathrm{C}$ using a sweep rate if $50 \mathrm{mVs}^{-1}$ Current densities reflect the geometric surface area of a $5.56 \mathrm{~mm}$ glasssy carbon disk .................................................................. 107

Figure 3.3 The theoretical $\Delta \mu$ signatures for each adsorbed halide on the surface of $\mathrm{Pt}_{6}$ cluster in four possible adsorption sties: a)atop, b) bridged, c) 3-fold (fcc) and d) hexagonal closed pack (hcp). $\Delta \mu_{\mathrm{t}}=\mu\left(\mathrm{Pt}_{6}-\mathrm{X}\right)-\mu\left(\mathrm{Pt}_{6}\right)$; where $\mathrm{X}$ is $\mathrm{Cl}^{-}, \mathrm{Br}^{-}$and $\mathrm{I}^{-}$. The Pt-X bond distances are calculated based on atomic radii, and are: $2.35 \mathrm{~A}^{\circ}$, $2.50 \mathrm{~A}^{\circ}$ and $2.75 \mathrm{~A}^{\circ}$ for Pt-Cl, Pt-Br and Pt- I respectively.

Figure 3.4 Theoretical $\Delta \mu$ signatures for Oxygen adsorption on $\mathrm{Pt}_{6}$ cluster in atop, bridged, fcc and hep configurations

Figure 3.5 Pt $\mathrm{L}_{3}$ edge $\Delta \mu=\mu(\mathrm{V}$, halide $)-\mu(0.54 \mathrm{~V}$ clean $)$ spectra for $30 \mathrm{wt} \% \mathrm{Pt} / \mathrm{C}$ in $1 \mathrm{M}$ $\mathrm{HClO} 4$ and $10 \mathrm{mM}$ of b) chloride, c) bromide and d)iodide. 5a illustrates $\Delta \mu$ plots in a $1 \mathrm{M} \mathrm{HClO} 4$ electrolyte with no added halide.

Figure 3.6 Plot of $\Delta \mu$ amplitudes for all three halides at a) $1 \mathrm{mM}$ and b) $10 \mathrm{mM}$ concentrations versus potential (vs. RHE). $\Delta \mu$ amplitudes for clean electrolyte $\left(1 \mathrm{M} \mathrm{HClO}_{4}\right)$ is also given in both cases for comparison purposes. All data has been normalized to 0.08 at $0.05 \mathrm{~V}$, as discussed in text 
Figure 3.7 Theoretical $\Delta \mu_{\mathrm{t}}=\mu\left(\mathrm{Pt}_{6}-\mathrm{X}+\mathrm{F}\right)-\mu\left(\mathrm{Pt}_{6}\right)$ signatures; where $\mathrm{X}$ is $\mathrm{Cl}^{-}$, $\mathrm{Br}^{-}$and $\mathrm{I}^{-}$ in a),b) and c) respectively.

Figure 3.8a and b. Pt $\mathrm{L}_{3}$ edge $\Delta \mu=\mu(\mathrm{V}$, halide $)-\mu(0.54 \mathrm{~V}$ clean $)$ spectra for $30 \mathrm{wt} \%$ $\mathrm{Pt} / \mathrm{C}$ in $1 \mathrm{M} \mathrm{HClO} 4$ and $10 \mathrm{mM}$ of a) bromide and b)iodide, clean spectrum in this case is XAS spectrum collected at $0.54 \mathrm{~V}$ in the same electrolyte as spectra collected at other potentials, Figure 8c shows $\Delta \mu$ amplitudes vs. potential correlating with parts b and

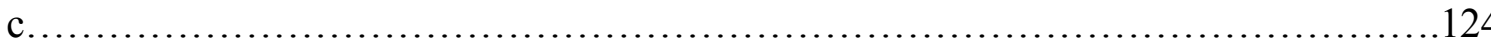

Figure 4.1 Methanol and $\mathrm{O}_{2}$ flow and the reactions occurring at each electrode in the DMFC studied in this work...................................................... 138

Figure 4.2 Plot of cell potential vs current density for the two different $\mathrm{O}_{2}$ gas flows as indicated. Also sketched are the estimated cathode and anode potentials.

Figure 4.3: a) Theoretical signatures obtained from FEFF8 calculations for $\mathrm{OH}$ and $\mathrm{CO}$ in an atop site on a $\mathrm{Pt}_{6}$ cluster as illustrated. Also shown is $\Delta \mu$ data reported by Scott et al at the anode in methanol at $700 \mathrm{mV}$ illustrating the 3 separate peaks, coming from $\mathrm{OH}$ near and far from a $\mathrm{Ru}$ island and $\mathrm{O}$ on $\mathrm{Pt}$ as illustrated. b) Experimental data from the anode at the bottom window (near the methanol inlet) at the indicated cell potentials. Four shaded bars indicate $|\Delta \mu|$ magnitudes (light for $\mathrm{O}(\mathrm{H})$ and dark for $\mathrm{CO}$ ) utilized to prepare Figure 4.5 with base estimated as discussed in text. .

Fig. 4.4: Plot of $\Delta \mu$ at the indicated cell potentials (in $\mathrm{mV}$ ) at the cathode outlet with 200 $\mathrm{ml} / \mathrm{min}_{2}$ flow rate and using $150 \mathrm{mV}$ as the reference potential in the $\Delta \mu$ calculation. Note the oscillatory behavior of $|\Delta \mu|$ with potential................................ 148

Figure 4.5: Plot of $|\Delta \mu|$ magnitudes vs cell potential for the indicated adsorbate species on the anode (left) and cathode (right) for the high $\mathrm{O}_{2}$ flow rate in the three separate windows

Figure 4.6: Plot of the previously reported $|\Delta \mu|$ after bubbling with $\mathrm{N}_{2}$ (only water activation) and $\mathrm{O}_{2}$ (water and $\mathrm{O}_{2}$ activation) in an electrochemical cell, and that for an operating fuel cell when full dynamic current is flowing. Also shown is the $\mathrm{OH}$ (blue dotted) and $\mathrm{O}_{2}$ (red dotted) coverage (in arbitrary units) predicted by the Wang et al model for ORR on $\mathrm{Pt}(111)$....

Figure 4.7: Exactly similar to Fig. 5 but now for low $\mathrm{O}_{2}$ flow rate. Note that the anode potential is now not give at the top because this now changes depending on the cathodic conditions as summarized in Figure 4.8. 
Figure 4.8 Qualitative estimates for cathode (solid) and anode (dotted) potentials as a function of cell potential at the top, middle, and bottom windows under low $\mathrm{O}_{2}$ flow conditions. Insert shows a model of segments (windows or resistors) connected in parallel. Also the estimated dominant adsorbates are indicated at the right for the corresponding electrode potential.

Figure 5.1 Left: $\mathrm{K}_{2} \mathrm{FeO}_{4}$ relative FTIR absorbance from 1300-1600 $\mathrm{cm}^{-1}$. Right: SEM of conventional and battery active nano $\mathrm{K}_{2} \mathrm{FeO}_{4}$

Figure 5.2 Discharge profile of super-iron batteries

Figure 5.3 Plot of A from EXAFS fit vs. difference in 1s to $3 \mathrm{~d}$ pre-edge peak; $\Delta \mu=\mu(\mathrm{t})$ $\mu(\gamma-\mathrm{Fe} 2 \mathrm{O} 3)$. Insert: plot of XAS data, $\mu(\mathrm{E})$ for the indicated samples, where the data for $\mathrm{K} 2 \mathrm{FeO} 4$ is for the undischarged milled cathode.............................. 176

Figure 6.1a) Comparative discharge of micron-sized $\mathrm{K}_{2} \mathrm{FeO}_{4}$ and $\mathrm{BaFeO}_{4}$ cathodes mixed with $10 \%$ carbon black in a $1 \mathrm{~cm}$ diameter coin cell, $\mathrm{Zn}$ anode and saturated $\mathrm{KOH}$ electrolyte. Discharged under constant load of $1000 \mathrm{ohm} . \mathrm{b})$ Energy capacity comparison of $\mathrm{K}_{2} \mathrm{FeO}_{4}$ and $\mathrm{BaFeO}_{4}$ with a $\mathrm{Zn}$ anode.

Figure 6.2 Residuals defined as described in text between the full discharge product for $\mathrm{nm}-\mathrm{K}_{2} \mathrm{FeO}_{4}$ and that after 8 hours and full discharge of $\mu \mathrm{m}-\mathrm{BaFeO}_{4}$ compared to the standards indicated.

Figure 6.3 Linear EXAFS component fits for two nm- $\mathrm{K}_{2} \mathrm{FeO}_{4}$ discharged cathodes: a) cathode discharged for 3 hours. b) cathode discharged for 16 hours....

Figure 6.4. XAS data, $\mu(\mathrm{E})$ for $\mathrm{BaFeO} 4$ and $\gamma-\mathrm{Fe}_{2} \mathrm{O}_{3}$ as standard, where the data for $\mathrm{BaFeO}_{4}$ is for the undischarged micron sized cathode

Figure $6.5 \Delta \mu$ results for $\mathrm{nm}_{-} \mathrm{K}_{2} \mathrm{FeO}_{4}$ at 3,5,8, and $16 \mathrm{hrs}$ discharge time under $600 \Omega$

load. The magnitudes of the $\Delta \mu(3 \mathrm{~d})$ and $\Delta \mu(\mathrm{ed})$ are indicated by the red arrow

Figure 6.6 Plot of the indicated $\Delta \mu$ amplitudes (at the edge and pre-edge peaks) and $\mathrm{A}$ (EXAFS) for the nm- and $\mu \mathrm{m}-\mathrm{K}_{2} \mathrm{FeO}_{4}$ as a function of discharge time. The light solid blue line on the $\mid \Delta \mu\left(\right.$ ed) $\mid$ plot suggest how it would change if just $\mathrm{H}^{+}$was intercalated. Measurements were taken on $50 \mathrm{mAh}$ cells with cathodes composed of $10 \%$ carbon black, $90 \%$ of $\mathrm{K}_{2} \mathrm{FeO} 4$ and $16 \mathrm{M} \mathrm{KOH}$ electrolyte and discharged under constant load of 600 ohms. Vertical line drawn at rearrangement..................................... 203

Figure 6.7 Plot of $\Delta \mu$ at the $3 \mathrm{~d}$ pre-edge and edge and A(EXAFS) vs. discharge time for $\mathrm{BaFeO}_{4}$. Vertical line drawn at rearrangement. 
Figure 6.8 Unit cell of crystalline $\mathrm{K}_{2} \mathrm{FeO}_{4}$ along a) $\mathrm{x}$ and b) y axes. Molecular structures program for Mac and Windows. CrystalMaker Software Ltd, Oxford,

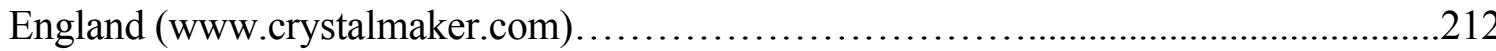

Figure 6.9. $\mathrm{K}_{2} \mathrm{FeO}_{4}$ and $\mathrm{BaFeO}_{4}$ Fourier transform prior to discharge; $\mathrm{BaFeO}_{4}$ shows increased $\mathrm{Fe}-\mathrm{O}$ and $\mathrm{Fe}-\mathrm{Fe}$ bond lengths and large $\mathrm{Fe}-\mathrm{O}$ peak width..................214

Figure 6.10. Comparative discharges of various $\mathrm{Fe}(\mathrm{VI})$ salt cathodes versus $\mathrm{Zn}$ with

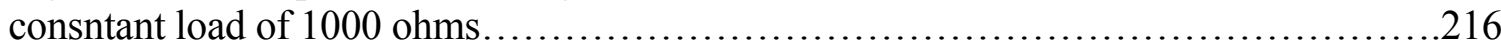




\section{List of Tables}

Table 1.1 Different types of fuel cells, their operating temperature and application. Adapted from Fuel Cell Systems Explained, by Larminie et. al. ...........................3

Table 1.2. Comparison of Various Characterization Techniques for Fuel Cell Catalyst, adapted from work by Roth et. al.

Table 1.3. Comparison of various $\mathrm{Fe}(\mathrm{VI})$ salts charge storage and intrinsic capacities of

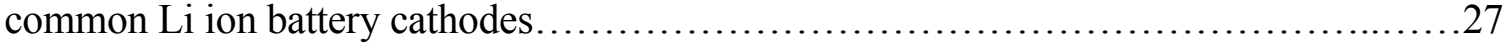

Table 2.1. X-ray absorption edges and their correlating core level excitations..............51

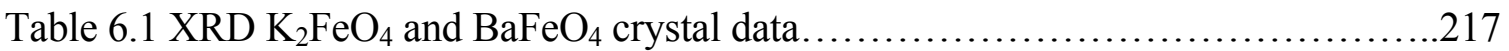




\section{Glossary of Terms}

AES: Auger Electron Spectroscopy

AFC: Alkaline Fuel Cell

AFM: Atomic Force Microscopy

ASAXS: Anomalous Small Angle X-ray Scattering

ATR: Attenuated Total internal Reflection

IRAS: Infra-Red Absorption Spectroscopy

CE: Counter Electrode

CV: Cyclic Voltammetry

DMFC: Direct Methanol Fuel Cell

ECTDMS: Electrochemical Thermal Desorption Mass Spectroscopy

EDS: Energy Dispersive X-ray Spectroscopy

EASA: Electrochemically Active Surfcae Area

EXAFS: Extended X-ray Absorption Fine Structure

FTIR: Fourier Transform Infra Red

GDL: Gas Diffusion Layer

HEV: Hybrid Electric Vehicle

HOC: Highly Ordered Commensurate

HOR: Hydrogen Oxidation Reaction

HREELS: High Resolution Electron Energy Loss Spectroscopy

$\mathrm{H}_{\text {upd }}$ : Hydrogen Underpotential Deposition

xxiii 
LEED: Low Energy Electron Diffraction

MCFC: Molten Carbonate Fuel Cell

MEA: Membrane Electrolyte Assembly

MOR: Methanol Oxidation Reaction

NMR: Nuclear Magnetic Resonance

ORR: Oxygen Reduction Reaction

PAFC: Phosphoric Acid Fuel Cell

PEM: Polymer Electrolyte Membrane

PHEV: Plug-in Hybrid Electric Vehicels

PIPS: Passivated Implanted Planar Silicon

PLD: Pulsed Lased Deposition.

ppm: Parts per Million

RDE: Rotating Disk Electrode

RHE: Reversible Hydrogen Electrode

RRDE: Rotating Ring Disk Electrode

SEI: Solide Electrolyte Intreface

SEM: Scanning Electron Microscopy

SFG: Sum Frequency Generation

SHE: Standard Hydrogen Electrode

SHG: Second Harmonic Generation

SNIFTIRS: Subtractively Normalized Infra-Red Absorption Spectroscopy

SOFC: Solid Oxide Fuel Cell 
SPM: Scanning Probe Microscopy

STM: Scanning Tunneling Microscopy

SXS: Surface X-ay Scattering

TEM: Transmission Electron Micrsocopy

TPD: Temperaute Programmed Desorption

UHV: Ultra-high Vacuum

UPD: Underpotential Deposition

WE: Working Electrode

XANES: X-ray Absorption Near Edge Structure

XAS: X-ray Absorption Spectroscopy

XPS: X-ray Photoelectron Spectroscopy

XRD: X-ray Diffraction 


\section{Chapter 1: Introduction}

About $28 \%$ of the total energy consumption in the U.S. is used for transportation, and

the U.S. as of 2009 accounts for $19.4 \%$ of the cars registered worldwide ${ }^{1}$. The ever growing use of automobiles and consequent higher demands for automotive fuels presents the challenge of developing alternative energy sources that utilize sustainable and environmentally friendly fuels and use them more efficiently. This strategy must not only aim to resolve climate change through reduction of the carbon footprint (i.e, reduced emission of greenhouse gases such as $\mathrm{CO}_{2}$ and other hydrocarbons) but also reduce U.S. dependence on foreign oil, which potentially can decrease national security concerns. Various alternative fuels and energy storage and conversion devices that use them more efficiently have been considered for development of zero or at least low emission vehicles.

Figure 1.1 illustrates Thomas's projections for various alternative vehicle options if only these scenarios are applied to light-duty vehicles, in the $21^{\text {st }}$ century. ${ }^{2}$ As indicated in this figure, both hybrid electric vehicles (HEV) and plug-in hydride electric vehicles (PHEV) will reduce greenhouse gas emissions. However, these two types of vehicles are still dependent on the internal combustion engine and cannot reduce the greenhouse gas emissions to less than $60 \%$ of 1990 levels, which is the goal defined by the climate change community. ${ }^{2,3}$ The only plausible scenario for successful reduction of the carbon footprint is by replacing internal combustion engines with fuel cells and/or batteries. As such, the development and successful commercialization of both fuel cells and batteries as alternative power sources is vital in the gradual replacement of combustion engines and 
Greenhouse Gas Pollution (Light duty vehicles only) (Billion/ tonnes CO2-equivalent/year)

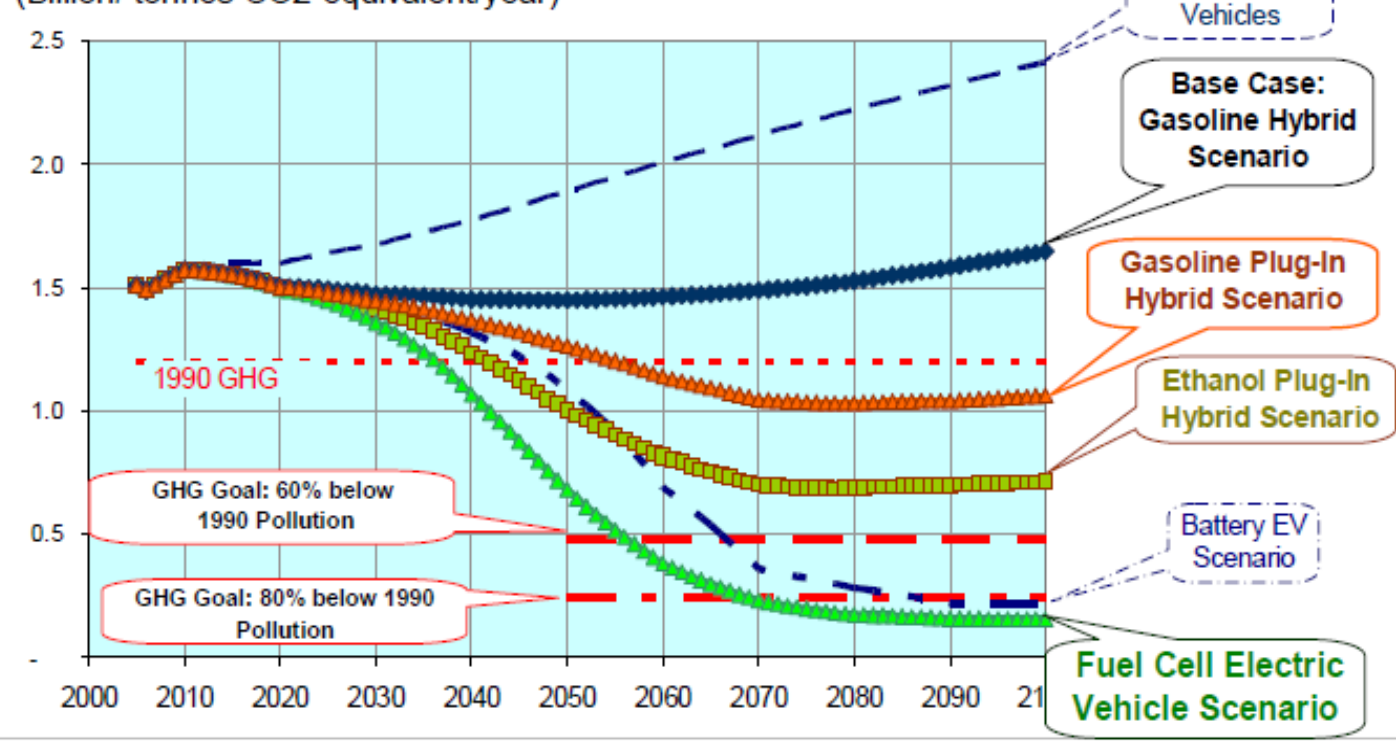

Figure 1.1 Projection of greenhouse gas pollution levels in U.S. over the $21 \mathrm{st}$ century by light duty vehicles only. Figure adapted from a study by C.E.S. Thomas. ${ }^{2}$ 
reduction of the global carbon footprint. However, both of these alternative energy devices still face many challenges to commercialization and widespread global application.

The development of sustainable and low cost materials for use as electrodes in fuel cells and batteries, and controlling the surface reactions at these electrodes that affect the overall performance of these electrochemical cells, are two of the hurdles that the transportation industry faces. The focus of this dissertation is on characterizing the electrochemical processes occurring at the electrode/electrolyte interface in both fuel cells and batteries utilizing X-ray absorption spectroscopy (XAS).

In this chapter, an overview of the working principles of both fuel cells and batteries will be given as well as highlighting the challenges existing with both of these devices that arise from these surface electrochemical reactions. This is followed by a short review of the characterization techniques appropriate for studying these electrochemical processes.

\section{1 Fuel Cells}

Both fuel cells and batteries are galvanostatic cells that consist of an anode and a cathode and the electrolyte. In both of these devices, and in all electrochemical cells under galvanic current, oxidation reactions take place at the anode and reduction reactions take place at the cathode. In a fuel cell, the anode is where the oxidation of the fuel such as $\mathrm{H}_{2}$ occurs and the cathode is where the oxygen reduction reaction (ORR) occurs. Thus, in fuel cells the cathode is defined as the positive electrode and the anode as the negative electrode, with electrons moving from the negative to positive electrode through the external circuit. In batteries, however the polarization of the anode and cathode differ during charge and discharge. During the discharge (under galvanic current), electrons flow 
from the anode to the cathode through the external circuit. However, the electron flow direction is reversed in the charging cycle of a battery. During the charging process, the current is produced by the applied potential, thererfore the current is electrolytic. Now, the electrode polarity is reversed, where the anode is the positive electrode and the cathode is the negative electrode.

Fuel cells "convert" chemical energy to electrical energy; the fuel on the anode side and oxygen on the cathode side continuously provided. Since fuel cells are "open systems" with the reactants fed continually, their capacity is limited only by the amount of fuel stored in an external tank. To our knowledge, the first ever demonstration of a fuel cell was conducted by lawyer and scientist William Grove in 1839. ${ }^{4}$ Although the operational basics of fuel cells were known since the 19th century, they did not gain attraction and application in industry until the late 1950s when scientists at General Electric modified the fuel cell design drastically by designing the sulphonated polystyrene ion-exchange membrane and electrocatalyst. ${ }^{5}$ This led to the utilization of fuel cells by NASA in the Gemini and Apollo missions in the 1960s and 1970s. NASA allocated funds for developing "space qualified" fuel cells, which resulted in a massive increase in interest and research on fuel cells. These efforts led to new designs and use of novel materials in fuel cells that increased their stability, reduced their costs and transformed them into smaller and more portable devices.

Extensive research aimed to resolve the main fuel cell challenges over the years has led to the development of a variety of fuel cell types. Table 1.1 illustrates the main classes of fuel cells employed today. ${ }^{4}$ The main technical challenges to the development of fuel 


\begin{tabular}{lccc}
\hline Fuel cell type & Mobile ion & $\begin{array}{c}\text { Operating } \\
\text { temperature }\end{array}$ & Applications and notes \\
\hline $\begin{array}{c}\text { Alkaline (AFC) } \\
\text { Proton exchange } \\
\text { membrane } \\
\text { (PEMFC) }\end{array}$ & $\mathrm{OH}^{-}$ & $50-200^{\circ} \mathrm{C}$ & $\begin{array}{c}\text { Used in space vehicles, e.g. Apollo, Shuttle. } \\
\text { Vehicles and mobile applications, and for } \\
\text { lower power CHP systems }\end{array}$ \\
$\begin{array}{c}\text { Direct methanol } \\
\text { (DMFC) }\end{array}$ & $\mathrm{H}^{+}$ & $20-90^{\circ} \mathrm{C}$ & $\begin{array}{c}\text { Suitable for portable electronic systems of low } \\
\text { power, running for long times }\end{array}$ \\
$\begin{array}{c}\text { Phosphoric acid } \\
\text { (PAFC) }\end{array}$ & $\mathrm{H}^{+}$ & $\sim 220^{\circ} \mathrm{C}$ & $\begin{array}{c}\text { Large numbers of 200-kW CHP systems in use. } \\
\text { Molten carbonate } \\
\text { (MCFC) }\end{array}$ \\
$\begin{array}{c}\text { Solid oxide } \\
\text { (SOFC) }\end{array}$ & $\mathrm{CO}^{2-}$ & $\sim 650^{\circ} \mathrm{C}$ & $\begin{array}{c}\text { Suitable for medium- to large-scale CHP } \\
\text { systems, up to MW capacity }\end{array}$ \\
\hline
\end{tabular}

Table 1.1 different types of fuel cells, their operating temperature and application. Adapted from Fuel Cell Systems Explained, by Larminie et. al. ${ }^{4}$ 
cells for practical energy conversion devices (aside from cost effectiveness) are to increase the oxygen reduction reaction rate, and consequently the current density and power output, to find sufficiently durable catalysts that will outlive the usual lifetime of an automobile, and to find an ideal fuel. This dissertation focuses on characterization of the electrochemical processes in Polymer Electrolyte Fuel Cells (PEM) and Direct Methanol Fuel Cells (DMFC). In the next sections, the basic operational principles and main technical challenges of these two types of fuel cells will be discussed in detail.

\subsubsection{Basic Operation Principles of Fuel Cells}

As was mentioned previously, fuel cells are comprised of an anode which is fed constantly with a supply of hydrogen or hydrogen-base fuel (i.e., methanol, ethanol) and a cathode with a continuous flow of an oxidant (i.e. oxygen). Figure 1.2 shows a simplified diagram of a polymer electrolyte fuel cell. The electrical energy is produced by electrochemical oxidation of fuel and electrochemical reduction of oxygen. A "three-phase boundary" is formed at the intersection of the reactants (which are in the gaseous state), the electrolyte (liquid phase) and the catalyst (solid phase). This three phase boundary plays a significant role in the performance of the fuel cell. ${ }^{6}$ Common problems observed at the three phase boundary that affect the current output are: 1) low contact area between the gaseous reactants, electrolyte and electrode; 2) the high resistance of the electrolyte towards ion transport and the distance between the electrodes. To resolve these problems, flat and porous electrodes (i.e. catalysts) have been utilized. The porous electrode is coated with a thin layer of electrolyte on one side and exposed to gaseous reactants on the other side: the 


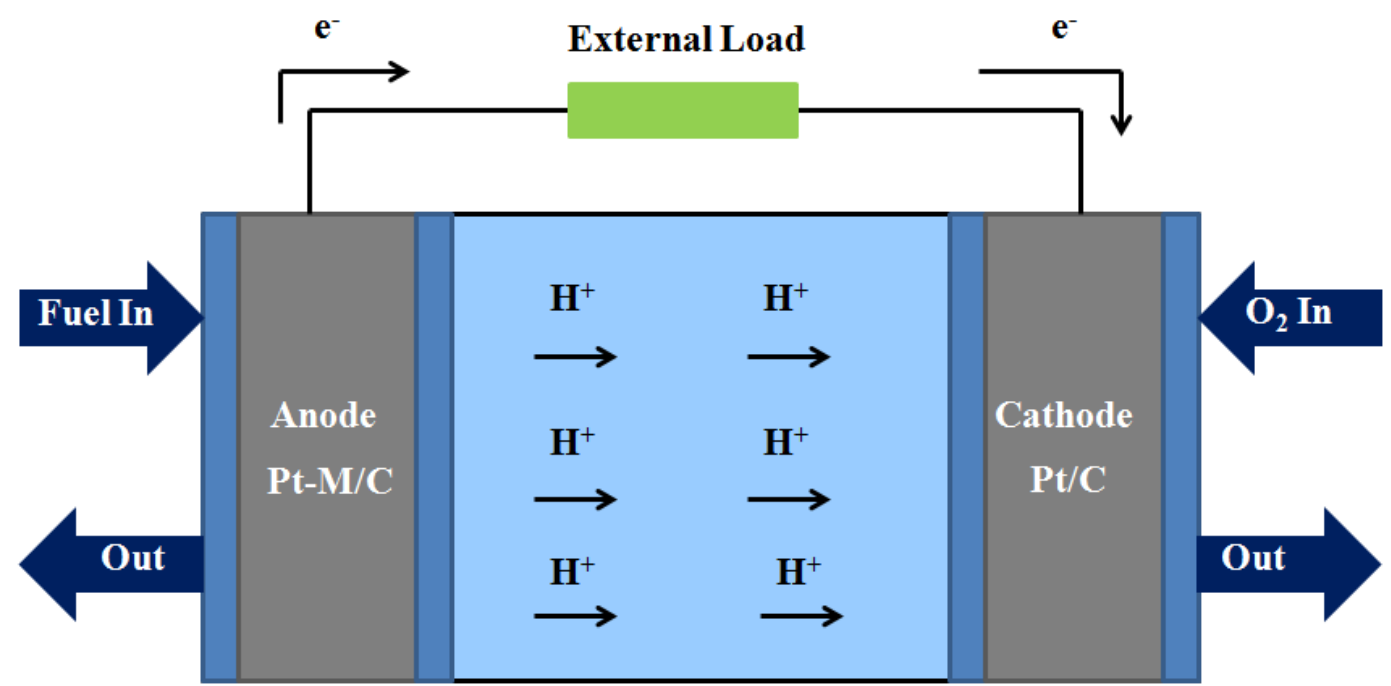

Proton Exchange Membrane

Figure 1.2 Schematic of a Proton Exchange Membrane (PEM) fuel cell.Adapted from Energy \& Environment, MIT Newsletter March 2005 
latter must diffuse through the electrode and the electrolyte. Close attention must be paid to the amount of electrolyte used to coat the electrode; too much electrolyte can "flood" the electrode and block diffusion of gaseous species to the reaction sites. ${ }^{4}$

To fully characterize the operational basics of the fuel cell, both the anode and cathode reactions should be closely studied. The anodic reaction depends on the type of fuel utilized in the cell; in this dissertation the focus is on hydrogen oxidation in a proton exchange membrane fuel cell (PEMFC) and methanol oxidation in a direct methanol fuel cell (DMFC). The reactions are as follows: ${ }^{7}$

Anode:

$\mathrm{H}_{2} \rightarrow \mathrm{H}^{+}+2 \mathrm{e}^{-}$

$\mathrm{CH}_{3} \mathrm{OH}+\mathrm{H}_{2} \mathrm{O} \rightarrow \mathrm{CO}_{2}+6 \mathrm{H}^{+}+6 \mathrm{e}^{-}$

Cathode:

$3 / 2 \mathrm{O}_{2}+6 \mathrm{H}^{+}+6 \mathrm{e}^{-} \rightarrow 3 \mathrm{H}_{2} \mathrm{O}$

Overall reaction:

$2 \mathrm{H}_{2}+\mathrm{O}_{2} \longrightarrow 2 \mathrm{H}_{2} \mathrm{O}$

$\mathrm{CH}_{3} \mathrm{OH}+3 / 2 \mathrm{O}_{2} \rightarrow \mathrm{CO}_{2}+2 \mathrm{H}_{2} \mathrm{O}$
$\mathrm{E}^{0}=0.00 \mathrm{~V} \quad$ PEMFC

$\mathrm{E}^{0}=0.046 \mathrm{~V}$ DMFC

$$
\mathrm{E}^{0}=1.23 \mathrm{~V}
$$

In both of these cases (PEMFC and DMFC), the electrons produced at the anode travel through the electrical circuit to the cathode, meanwhile $\mathrm{H}^{+}$flows through the electrolyte and the PEM from the anode to the cathode. The PEM itself contains $\mathrm{H}^{+}$ions. The electrons and incoming protons react with oxygen on the cathode side to produce water. The PEM should only allow $\mathrm{H}^{+}$flow through it and reject electrons so that the electrons will be forced to travel through the external circuit. In the case of methanol oxidation $\mathrm{CO}_{2}$ 
is also produced as a product of methanol oxidation, which cannot pass through the membrane, and remains in the anodic compartment. This product can be flushed out through methanol circulation. Note that for hydrogen oxidation in a PEMFC, 2 electrons are released at the anode for each $\mathrm{H}_{2}$, and two $\mathrm{H}_{2}$ are needed for each $\mathrm{O}_{2}$ molecule on the cathode side, where as for the DMFC 6 electrons are released at the anode per methanol molecule reacted.

\subsubsection{Fuel Cell Challenges}

As summarized above in Table 1, there are currently six types of fuel cells under development for possible future applications. This dissertation is focused on PEMFCs and DMFCs. Challenges pertaining to development and commercialization of these two types of fuel cells will be addressed here and indeed there are many challenges still to be resolved for the full commercialization of these two types of fuel cells:

1. Methanol oxidation at the anode in a DMFCs proceeds much more slowly compared to that of the $\mathrm{H}_{2}$ oxidation. Thus the DMFC results in a fuel cell with smaller power output for a certain given size. ${ }^{4}$

2. Further, both of these fuel cells still need to address the problem of cost effectiveness. One important way to decrease the cost is to reduce the catalyst loading.

3. Both cathode and anode catalysts suffer from loss of stability. The degradation of the catalysts and membrane over time can reduce the life span of the fuel cell. 
All of the above mentioned problems need to be resolved for fuel cells to gain widespread industrial application. In this dissertation, however, the main focus is on the poisoning of the electrocatalysts in both the anode and cathode sides and the inhomogeneous current distribution across the cross-sectional area of the fuel.

\section{a. Poisoning of the Pt Anode}

One major issue encountered in DMFCs is the poisoning of the anode catalyst by the methanol electro-oxidation reaction (MOR) intermediates and by-products. Relative to the Hydrogen Oxidation Reaction (HOR), overpotentials as large as $200-300 \mathrm{mV}$ have been observed, this resulting in part from the small area of "clean" catalysts remaining for the MOR. ${ }^{8}$ This issue also arises in $\mathrm{H}_{2}$ PEMFCs when reformate products from diesel, gasoline or natural gas are used as fuel, because small amounts of $\mathrm{CO}$ are present in the $\mathrm{H}_{2}$ produced in this process. $\mathrm{CO}$ is a species that is especially notorious for poisoning Pt based catalysts. Even amounts as low as $10 \mathrm{ppm}$ of residual $\mathrm{CO}$ in $\mathrm{H}_{2}$ can act a poison on the Ptbased anode. ${ }^{9,10}$ To oxidize off the CO poison, the anode potential needs to be increased to above $0.6 \mathrm{~V}$ (RHE). At this potential, a Langmuir-Hinshelwood surface reaction between $\mathrm{CO}_{\mathrm{ad}}$ and $\mathrm{OH}_{\mathrm{ad}}$ allows for $\mathrm{CO}$ to be oxidized to $\mathrm{CO}_{2}$ which moves away from the Pt surface. ${ }^{11}$ However, the rise of the potential to such levels will result in an unacceptable loss of cell voltage and efficiency. ${ }^{12}$

One approach to alleviate this problem is inclusion of other reactive metals on the electrode surface. Alloying Pt with transition metals such as $\mathrm{Ru}, \mathrm{Mo}$ and $\mathrm{Sn}$, improves the catalyst tolerance towards CO poisoning. ${ }^{13-20}$ Three main mechanisms have been suggested 
for the improvement of the catalyst performance when Pt is alloyed with transition metals, these mechanisms are dependent on the voltage range that the fuel cell is operating:

1. At potentials below $0.25 \mathrm{~V}$ vs. RHE, the bifunctional mechanism (BF) prevails. The bifunctional mechanism involves formation of $\mathrm{OH}$ from water activation on the transition metal islands. $\mathrm{OH}_{\mathrm{ad}}$ then oxidizes $\mathrm{CO}_{\mathrm{ad}}$ to $\mathrm{CO}_{2}$.

2. At potentials between 0.25 to $0.4 \mathrm{~V}$ vs. RHE, the direct ligand (DL) mechanism dominates. Here, $\mathrm{OH}$ formation from water activation happens directly on the Pt surface but close to the transition metal islands. The transition metal islands modify the electronic properties of the nearby Pt by contributing d-electron density.

3. At potentials above $0.4 \mathrm{~V}$ vs RHE, the direct (D) mechanism is dominant. Here, $\mathrm{OH}$ via water activation directly oxidizes the CO. ${ }^{21,22}$

Over the years, extensive research has been focused on developing more tolerant and less expensive anode catalysts.

\section{b. Poisonings of the Pt Cathode}

Both PEMFCs and DMFCs currently utilize catalysts that are based on precious metals, such as Pt and Pt alloys. While Pt shows high activities towards the HOR on the anode side, the oxygen reduction reaction (ORR) on the cathode side can experience overpotential losses of more than $200 \mathrm{mV}$. The slow ORR kinetics leads to a significant 


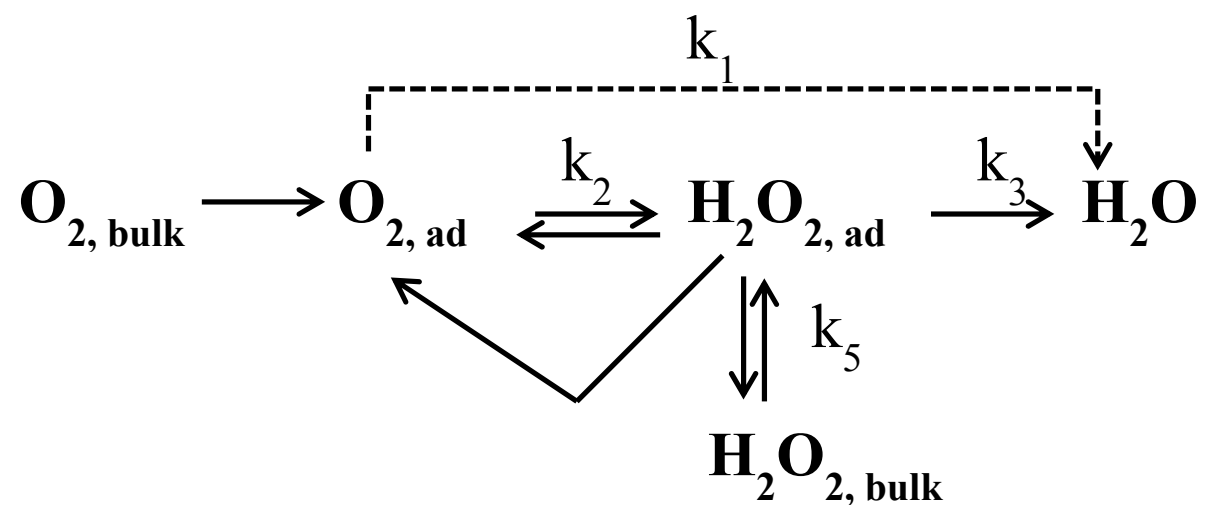

Figure 1.3 A simplified schematic of the ORR pathway; figure adapted from works by Paulus et al. and Wroblowa et. al. ${ }^{97}$ 
decrease in power output. Furthermore, the cathode performance can be affected by the presence of trace amounts of all kinds of impurities. Figure 1.3 shows one simplified scheme for the ORR reaction; namely, the dissociative mechanism (other mechanisms exist, as indeed the ORR mechanism after all these years of study is still controversial). Note that the ORR can either go through a direct four electron reduction pathway to form water or a series of reactions through an intermediate $\left(\mathrm{H}_{2} \mathrm{O}_{2}\right){ }^{22,}{ }^{23}$ The two electron pathway that involves $\mathrm{H}_{2} \mathrm{O}_{2}$ as the main product is not desirable since it can accelerate polymer membrane degradation and also decreases the fuel efficiency.

Impurities of many kinds (cations, anions or organic residues) can block reaction sites, which diminish the already slow ORR activity, and/or alter the reaction pathway that in turn can affect the product distribution. ${ }^{22}$ The corrosion of fuel cell stacks can produce many different reactant species that can reach the membrane electrolyte assembly (MEA) and the vital electrocatalyst, and therefore strict contamination control or build-up of corrosion resistance is necessary in PEMFCs.

An example of contamination anions are halides (i.e. $\mathrm{Cl}^{-}, \mathrm{Br}^{-}$and $\mathrm{I}^{-}$), which will be discussed in detail in Chapter 3 of this dissertation. The Pt catalyst's performance can seriously be affected by the presence of halides over the entire operational potential window. ${ }^{25}$ Adsorbed halides on the surface of a catalysts can change the reaction path and form $\mathrm{H}_{2} \mathrm{O}_{2}$ instead of the normal $\mathrm{H}_{2} \mathrm{O}$. It has been illustrated that $\mathrm{H}_{2} \mathrm{O}_{2}$ can also lead to the degradation of the PEM materials.$^{26}$ Therefore, the presence of halides is highly undesirable because it can adversely impact both the cell performance and life time. ${ }^{24}$ 
Cations can also contaminate the MEA and affect the cathode performance. They can degrade the membrane performance by ion-exchange with protons in the PEM as well as the ionomer that is distributed across the electrodes. ${ }^{27}$ This causes a decrease in the fuel cell's durability. Also cation deposition on the Pt cathode catalyst, in its operational voltage range, whether by Nernst deposition or under potential deposition (UPD), can reduce the ORR activity. For instance, it has been shown that $\mathrm{Cu}$ reduces the ORR activity on $\mathrm{Pt}$ at potentials below $0.8 \mathrm{~V}$ (vs. RHE). At higher current densities the decrease in ORR activity is severely problematic to the fuel cell performance. ${ }^{28,29}$

An extremely important cathode contamination problem observed in DMFCs is "methanol cross-over". This can also be observed in PEMFCs when they operate on incompletely reformatted methanol. In DMFCs, methanol on the anode side can mix readily with water, which is an essential component of the PEM electrolyte, and travel with water to reach the cathode side. The methanol that has crossed-over to the cathode will reduce the cathode Pt catalyst and cause mixed potentials. The superposition of methanol oxidation potential and the cathodic ORR potential, increases the cathode overpotential. Thus, the cathode performance is adversely affected, although no increase in $\mathrm{H}_{2} \mathrm{O}_{2}$ yield is observed. The overpotential losses due to methanol cross-over have been estimated to be around 100-120 mV. ${ }^{30-32}$ This overpotential loss, added to the already sluggish kinetics of the ORR, can severely impact the cell performance.

\section{c. Inhomogeneous Current Distribution}

Another challenge addressed in this thesis is the cross-sectional non-uniformities observed in the current distribution along a single cell in a DMFC. These current 
inhomogeneities can be caused by a number of reasons: variations in catalyst loading, fluctuations in feed supply, blocking or flooding of the MEA by gaseous or water products, and variations in temperature distributions. These variations in current distribution can potentially have adverse effects on the performance of the fuel cell; it can even cause the coexistence of local galvanic and electrolytic regions in a single channel of DMFCs. Chapter 4 of this dissertation aims at studying the spatial distribution of adsorbates on both anode and cathode at two oxygen flow rates. ${ }^{33-35}$

\subsubsection{X-ray Absorption Spectroscopy for Characterization of Fuel Cell Catalysts}

The challenges to commercialization from the perspective of the catalyst, suggest that understanding the reaction mechanisms on the electrodes can lead to design of more stable, durable and cost effective electrocatalyst. Techniques that can be used to study catalyst behavior under real operating conditions can especially provide researchers with valuable insight into the reaction mechanisms at the catalyst surface. There have been numerous studies characterizing the electrochemical reaction mechanisms occurring on the catalyst surface, but it is critical to know how a specific catalyst adapts and performs in a real fuel cell environment. For instance, in-situ X-ray absorption spectroscopy has shown that bimetallic Pt-Ru nanoparticles are extremely susceptible to changes in morphology in different environments: Pt tends to move to the particle surface when the catalyst is

exposed to $\mathrm{H}_{2}$, whereas $\mathrm{Ru}$ covers the surface in $\mathrm{O}_{2} .{ }^{36,37}$ Thus, a technique that can elucidate the electrochemical processes under operating conditions (in-operando) with 
element specificity and surface sensitivity is highly crucial in characterizing and providing feedback for designing better electroctalysts for fuel cells.

A variety of spectroscopic techniques have been used for this purpose over the years. Vibrational spectroscopic techniques such as Fourier transform infrared spectroscopy (FTIR), high resolution electron energy loss spectroscopy (HREELS) and optical sum frequency generation (SFG) can directly identify adsorption sites of molecules like $\mathrm{CO}$, but fail to provide information on adsorption and coverage of $\mathrm{O}$ and $\mathrm{OH}$. X-ray diffraction (XRD) can provide information on structure, particle size and phase identity but it is a longer range order technique and cannot provide adsorbate coverage information. In-situ XRD also has limitations for applications to very small particles. Electron spectroscopy techniques, such as Auger and X-ray Photoelectron Spectroscopy (XPS), can provide electronic information but require ultra-high vacuum conditions for operation. Table 1.2 compares a variety of techniques and their advantages/disadvantages for studying catalysts in fuel cells in-operando.

X-ray absorption spectroscopy (XAS) utilizes photons as probes, so it can provide both structural and electronic information. It is a short range order, element specific technique. Further, it can be utilized under reaction conditions. Utilizing the near edge region of XAS (XANES) and the extended fine structure section of the spectrum (EXAFS) enables researchers to follow both the adsorbate coverage and the structural and morphological changes to the catalysts at the same time. Over the last decade a large number of studies have been conducted on catalysts' characterization in fuel cells utilizing in-situ XAS. Insitu measurements can be carried out under various conditions: different temperatures, 


\begin{tabular}{|c|c|c|c|}
\hline Technique & Type of Information & Advantages/Disadvantages & In-Situ \\
\hline XAS & $\begin{array}{l}\text { Ads. binding site, } \\
\text { particle size }\end{array}$ & $\begin{array}{l}\text {-Synchrotron Required } \\
\text { - Complex theoretical analysis }\end{array}$ & Yes \\
\hline TEM & $\begin{array}{l}\text { Crystal structure, } \\
\text { cluster shape and size }\end{array}$ & $\begin{array}{l}\text { - Direct imaging of clusters } \\
\text { - Simultaneous imaging } \\
\text { - Diffraction and analysis } \\
\text { - HV required }\end{array}$ & Yes \\
\hline XRD & Crystal structure & $\begin{array}{l}\text { - Long range order } \\
\text { - Complex theoretical analysis }\end{array}$ & No \\
\hline ASAXS & Crystal structure & $\begin{array}{l}\text {-Synchrotron required } \\
\text { - Complex theoretical analysis }\end{array}$ & Yes \\
\hline XPS/AES & Electronic structure & - UHV required & No \\
\hline $\begin{array}{l}\text { TPD, } \\
\text { ECTDMS }\end{array}$ & Ads. binding energy & - UHV required & No \\
\hline ATR, IRAS & $\begin{array}{l}\text { Binding of atoms and } \\
\text { molecules }\end{array}$ & $\begin{array}{l}\text {-Noisy differential/difference } \\
\text { techniques } \\
\text {-3-fold site vibrations invisible }\end{array}$ & Yes \\
\hline IR/ Raman & $\begin{array}{l}\text { Binding of atoms and } \\
\text { molecules }\end{array}$ & $\begin{array}{l}\text { - Huge bulk electrolyte } \\
\text { absorption }\end{array}$ & Yes \\
\hline HREELS & $\begin{array}{l}\text { Binding of atoms and } \\
\text { molecules }\end{array}$ & - UHV required & No \\
\hline $\begin{array}{l}\text { SHG,SFG, } \\
\text { SNIFTIRS }\end{array}$ & $\begin{array}{l}\text { Binding of atoms and } \\
\text { molecules }\end{array}$ & $\begin{array}{l}\text {-Noisy differential/difference } \\
\text { techniques } \\
\text { - 3-fold site vibrations invisible }\end{array}$ & Yes \\
\hline NIS & $\begin{array}{l}\text { Binding of atoms and } \\
\text { molecule }\end{array}$ & $\begin{array}{l}\text { - Mirror surfaces require } \\
\text { - High surface areas required } \\
\text { due to small cross section for } \\
\text { scattering }\end{array}$ & No \\
\hline $\begin{array}{l}\text { DRIFTS, } \\
\text { FTIR-DRS }\end{array}$ & $\begin{array}{l}\text { Binding of probe } \\
\text { molecules }\end{array}$ & $\begin{array}{l}\text { - Cannot see atomic } \\
\text { adsorbates such as } \mathrm{O} \text { and } \mathrm{H} \\
\text { - Requires fine powder samples }\end{array}$ & Yes \\
\hline SPM & Electronic structure & $\begin{array}{l}\text { - 3D Surface profile with high } \\
\text { Resolution } \\
\text { - Maximum image size small }\end{array}$ & No \\
\hline
\end{tabular}

Table 1.2. Comparison of Various Characterization Techniques for Fuel Cell Catalyst, adapted from work by Roth et. al. ${ }^{98}$ 
different matrices, different pressure, etc. Thus, it is a highly versatile technique to probe a variety of issues in fuel cells as the fuel cell is operating. This technique can be coupled with a variety of electrochemical techniques such as voltammetry or chronoamperometry. These latter techniques enable researchers to follow electrochemical processes on the surface of the electrode as they proceed.

\subsection{Batteries}

Batteries are electrochemical cells that also convert chemical energy to electric energy. They are similar to fuel cells in that they are also galvanic cells that consist of an anode (negative electrode) and a cathode (positive electrode) and an electrolyte. Their difference with fuel cells is in that they are a "closed" system, meaning that the amount of reactants are restricted and the capacity of these electrochemical cells is limited by the material in the enclosure. Thus, batteries are "energy storage" devices. Batteries can be primary or secondary (rechargeable). In case of rechargeable batteries, the redox reaction can be reversed, and the battery can be "recharged". The principle components of a battery are similar to fuel cells, yet this is a completely different system as the fuel and oxidant are confined and there are no inlet/outlets for the reactants.

Batteries have played a crucial role in advancement of the clean and renewable energy industry, and in reduction of $\mathrm{CO}_{2}$ emissions around the world. Batteries such as the Li-ion and Ni-MH cells, have been utilized as energy sources for portable devices such as laptops and cell phones. Recently, Li-ion batteries have been utilized extensively as energy sources in vehicle electrification. For instance, last year Nissan introduced their full electric vehicle 
that relies on a $24 \mathrm{kWh} \mathrm{Li-ion} \mathrm{battery} \mathrm{to} \mathrm{store} \mathrm{its} \mathrm{energy} \mathrm{to} \mathrm{power} \mathrm{a} 80 \mathrm{~kW}$ AC motor with $48 \mathrm{Li}$-ion modules. Each module consists of four Li-ion battery cells that provide $207 \mathrm{lb}-\mathrm{ft}$ and 107 horsepower. ${ }^{38}$ A recent study has projected that Li-ion battery sales will grow by $600 \%$ up to 2015 , as more companies will be interested in production of hybrids, plug-ins and battery electric vehicles. Li-ion battery sales are predicted to increase from $\$ 9$ billion to $\$ 50$ billion worldwide by $2020 .{ }^{39}$

Li-ion batteries are comprised of a cathode (usually a Li metal oxide such as $\mathrm{LiCoO}_{2}$ ), an anode (usually a carbonaceous material such as graphite), and a carbonate-based organic electrolyte which contains Li-ion salts $\left(\right.$ such as $\left.\mathrm{LiPF}_{6}\right)$. During the discharge cycle, Li-ions are intercalated from the oxide cathode to the carbon anode through the electrolyte, then during the charging cycle Li-ions are deintercalated back to the cathode. This electrochemical reversible reaction occurs typically at around $4 \mathrm{~V}$ vs. the $\mathrm{Li} / \mathrm{Li}^{+}$electrode for the cathode and $0 \mathrm{~V}$ for the anode.

The electrolyte separates the ionic and electronic transport. The electrode material should be able to allow the flow of both Li ions and electrons. This means that the electrode material should have both ionic and electronic conductivity. To increase the electron conductivity of electrode materials in cathodes, usually a highly electronically conductive material such as carbon black is added to the active material along with a binder. The electrode active material should be homogenously mixed with the conductive matrix and the binder, and be in good contact with the electrolyte for the redox reaction to happen. Therefore, the electrode material is usually a porous composite. Another component of a 
battery is a porous separator material between the cathode and anode; this material allows for ions to flow through but prevents electrical short. ${ }^{40}$

The main challenges for development of batteries as energy storage devices for electric vehicles are the cost, safety, cell energy density (voltage/capacity), charge/discharge rates and short life times. To increase the capacity, that is the rate capabilities and power output of these state-of-the-art batteries, extensive research has been conducted throughout the world to design and modify all the components of the battery; however, the main focus of this dissertation is on characterizing novel, high capacity "cathode" materials.

\subsubsection{Novel Cathode Materials for High Capacity Batteries}

What kind of requirements should an ideal cathode fulfill?

- An ideal cathode should be comprised of a material which can easily be reduced and oxidized such as transition metals.

- The reduction/oxidation of the cathode material should have a high reversibility, especially when intercalating Li ion.

- The ideal cathode material should react with the anode with a high free energy of reaction. The higher the energy of the cathode oxidation/reduction reaction, the higher the capacity of the cathode material. Fulfilling these criteria also ensures that the output voltage against the anode oxidation transition is high in the case of the $\mathrm{Li}^{+} / \mathrm{Li}$ transition around 4.0 volts.

- High power density is also of crucial importance in electric car applications, as high charge/discharge rates are required for charging of batteries using regenerative braking. 
- The cathode material should have high structural stability during the charge/discharge cycles. It is also desirable to minimize the amount of inactive material inside the cathode composite, as a high percentage of inactive material inside the cathode (i.e., carbon black for increased electronic conduction and binders) lowers the energy density of the cathode, and this is important for transportation purposes.

- The ideal cathode material should have low cost and be environmentally benign.

Over the years, a variety of materials have been utilized as cathodes. With the growing demand for higher capacity energy storage devices, the specific tailoring of novel cathode materials that would provide ideal capacities and power densities is increasingly being utilized. A brief overview of the various classes of cathodic compounds utilized will be presented below, followed by a more in-depth look at multi-electron transfer cathodes, specifically "super-iron" cathodes.

\subsubsection{Classes of Cathode Materials}

To date, lithium metal oxides are the most widely used cathode materials. ${ }^{41}$ They can be divided into four main categories: 1) layered $\mathrm{LiMO}_{2}$ compounds (where $\mathrm{M}$ is a transition metal); 2) $\mathrm{LiM}_{2} \mathrm{O}_{4}$ compounds with spinel structure; 3) polyanion based compounds $\mathrm{Li}_{\mathrm{X}} \mathrm{M}_{\mathrm{Y}}\left(\mathrm{XO}_{4}\right)_{\mathrm{z}}$ ( where $\mathrm{M}$ is a transition metal and $\mathrm{XO}_{4}$ could be a variety of anions), and 4) multi-electron cathode materials. This dissertation is focused on development of multi electron transfer materials

\section{- Layered $\mathrm{LiMO}_{2}$ Cathodes:}

$\mathrm{LiCoO}_{2}$ is the most commercialized layered compound as a cathode material. It has a

high theoretical capacity $\left(274 \mathrm{mAhg}^{-1}\right)$. However, this compound suffers from poor 
structural stability caused by formation of Li-poor phases, which results in electrolyte decomposition and loss of reversible capacity to about half of the theoretical value. ${ }^{42}$ Moreover, cobalt is costly and not as readily available as other elements such as $\mathrm{Mn}$ or $\mathrm{Ni}$, and is also toxic in various oxidation states. Other candidates include $\mathrm{LiMnO}_{2}, \mathrm{LiNiO}_{2}$ and $\mathrm{LiFeO}_{2}$, which also have the same $\alpha-\mathrm{NaFeO}_{2}$ structure as $\mathrm{LiCoO}_{2}$. These compounds utilize more abundant, cheaper and less toxic elements. However, these alternative candidates suffer from low thermodynamic stability and are difficult to synthesize. As an alternative, extensive research has been conducted on $\mathrm{LiNi}_{\mathrm{x}} \mathrm{M}_{1-\mathrm{x}} \mathrm{O}_{2}$ (M-transition metal) layered compounds. ${ }^{43-45}$ Researchers have introduced various modifications to the crystal structure of these compounds by modifying the intralayer or disordering the structure, and thereby obtained higher capacities and rate capabilities compared to the classical $\mathrm{LiCoO}_{2}$ cathode material.

\section{- $\mathrm{LiM}_{2} \mathrm{O}_{4}$ (spinel structure) Cathodes:}

The other main category of cathode compounds are spinel compounds such as $\mathrm{LiMn}_{2} \mathrm{O}_{4}$. The advantages of these spinel structures include higher safety and intrinsic rate capabilities, due to the more chemically stable $\mathrm{Mn}^{3+} \mathrm{Mn}^{4+}$ redox couple and the 3D intercalation channels that facilitate Li ion diffusion. However, the Jahn-Teller distortion around $\mathrm{Mn}^{3+}$ causes a gradual capacity loss and a high possibility of Mn dissolution into the electrolyte. Doping Li on octahedral sublattice sites to produce Li-rich spinel compounds inhibits the Mn dissolution but at a capacity loss. ${ }^{46,47}$ 


\section{- Polyanion Cathodes $\left(\mathrm{Li}_{\mathrm{X}} \mathrm{M}_{\mathrm{Y}}\left(\mathrm{XO}_{4}\right)_{\mathrm{Z}}\right)$}

The third category of cathode compounds are polyanion based compounds such as $\mathrm{Li}_{\mathrm{X}} \mathrm{M}_{\mathrm{Y}}\left(\mathrm{XO}_{4}\right)_{2}$, where $\mathrm{M}$ is a transition metal and $\mathrm{X}$ can be either a group IV or $\mathrm{V}$ nonmetal such as $\mathrm{Si}, \mathrm{S}$ or $\mathrm{P}$, or another transition metal such as W or Mo. These compounds are regarded as highly promising electrodes for electric vehicle applications. The most prominent oxide of this type is $\mathrm{LiFePO}_{4}$. The fact that it is an iron oxide is a great advantage, since it utilizes an environmentally abundant and cheap metal that offers a high capacity $\left(170 \mathrm{mAhg}^{-1}\right)$. Further, $\mathrm{LiFePO}_{4}$ shows high reversibility and safety. The discharge potential for this compound is about $3.4 \mathrm{~V}$. vs. Li, and capacity fade even after several hundred cycles is minimal. ${ }^{48}$ The performance if this material is however limited by its low electronic conductivity $\left(10^{-9} \mathrm{~S} \mathrm{~cm}^{-1}\right)$ and very low lithium diffusivity $\left(10^{-10}\right.$ to $\left.10^{-16} \mathrm{~cm}^{2} \mathrm{~s}^{-1}\right){ }^{48-51}$ Doping $\mathrm{LiFePO}_{4}$ with carbon-gel during synthesis has been proposed as one strategy to increase the electronic and ionic conductivity of this material. ${ }^{52,53}$ One main strategy to increase the rate capabilities of this compound is to move to the "nano" domain. Nano-LiFePO4 particles when placed in a composite with carbon exhibit high rate performances, higher columbic efficiency and negligible capacity fade over 1100 cycles. $^{54,55}$

A variety of other metal oxides from this group of cathode materials has been explored over the years by many researchers: for instance olivine phase $\mathrm{LiCoPO}_{4}$ and $\mathrm{LiMnPO}_{4}$ were investigated and were shown to have smaller theoretical capacities but higher discharge voltages and higher energy densities. However, the low intrinsic ionic and electronic conductivity and large anisotropic lattice mismatch observed between charged 
and discharged states contribute to capacity retention and poor kinetics. ${ }^{56-58}$ Other strategies for designing high capacity olivine phase compounds include designing mixed-metal olivine compounds. Also, other polyanionic compounds such as flourophosphates ${ }^{59}$, flourosulphates $^{61}$ and silicates ${ }^{60}$ were explored as possible cathodes. Utilizing other anions can drastically change the electrode properties through lattice structure changes and charge balance differences. Finally, another major drawback of polyanionic cathodes compared to classical $\mathrm{LiCoO}_{2}$ is their common low material packing density, which limits their use in large battery applications. ${ }^{42}$

\section{- Multi-electron Cathodes:}

The specific energy density for a battery is:

$$
\mathrm{ED}=\mathrm{nFE} \mathrm{E}^{0} / \mathrm{M}_{\mathrm{i}}
$$

where ED is the energy density either per unit of weight $\left(\mathrm{Whkg}^{-1}\right)$ or unit of volume $\left(\mathrm{WhL}^{-}\right.$ $\left.{ }^{1}\right), \mathrm{n}$ is the number of electrons transferred per mole of reactants, $\mathrm{F}$ is Faraday's constant, $\mathrm{E}^{0}$ is the electromotive force of the reaction, and $\quad M_{i}$ is the sum of the formula weights of the active materials. Therefore, to increase the energy density of the cathode, lighter materials should be utilized, a redox couple with higher electromotive force should be employed, or a larger number of electrons per molecules should be transferred. ${ }^{62}$

The efforts described above for designing various cathode materials focused on finding redox couples with higher potentials. However, another effective strategy is to develop new cathodes allowing multi-electron transfer; i.e. more than one electron per formula unit. Most commercialized cathode materials offer redox reactions transferring only a single electron or even less than one electron per formula unit within the operating potential range 
of the battery. Above, we discussed several classes of cathode materials mainly comprised of various Li metal oxides or oxyphosphates. Most of these compounds are limited by the Li intercalation stoichiometry.

To design cathodes allowing a higher number of electrons to transfer per formula unit requires one to focus on new classes of materials. One class of multi-electron cathodes investigated by various researches is the metal fluoride based materials. Most of these metal fluoride compounds go through a reversible electrochemical "conversion" reaction (rather than intercalation) as they charge/discharge:

$$
\mathrm{xLi}^{+}+\mathrm{xe}^{-}+\mathrm{MF}_{\mathrm{x}} \rightarrow \mathrm{xLiF}+\mathrm{M}
$$

It is highly unlikely that this is a one step process, rather most of these fluorides show multiple steps in their charge/discharge curves which is indicative of a multistep conversion process. $^{63-65}$ It is more likely that there is a one-electron Li intercalation step followed by a multi-electron conversion step. This presents a problem with this class of compounds: the output potential of the intercalation step that occurs at higher valence states of the metal is much higher than the output potential of the conversion step, and this causes large power output fluctuations. ${ }^{63}$

\subsubsection{Super Iron salts}

Super-Iron batteries are a novel class of multi-electron transfer cathodes introduced by Licht et. al in 1999. ${ }^{66}$ Since these are highly oxidized iron salts (up to hexavalent oxidation states) they are often called "super-iron" salts. Since their introduction as battery materials in 1999, they have been investigated as cathodes in both aqueous and non-aqueous 
batteries. These super-oxidized salts discharge via a nominal 3 electron charge transfer, thus they could be viable candidates for "multi-electron" cathodes. Further, they are environmentally benign and their discharge product is ferric oxide which is non-toxic. They are also relatively inexpensive because iron is the second most abundant metal in the earth's core.

Various $\mathrm{Fe}(\mathrm{VI})$ salts have been synthesized and utilized as cathode compounds over the years: $\mathrm{Li}_{2} \mathrm{FeO}_{4}, \mathrm{~K}_{\mathrm{x}} \mathrm{Na}_{(2-\mathrm{x})} \mathrm{FeO}_{4}, \mathrm{~K}_{2} \mathrm{FeO}_{4}, \mathrm{Rb}_{2} \mathrm{FeO}_{4}, \mathrm{Cs}_{2} \mathrm{FeO}_{4}, \mathrm{SrFeO}_{4}, \mathrm{BaFeO}_{4}$ and $\mathrm{Ag}_{2} \mathrm{FeO}_{4}$.

The theoretical capacities of these salts are much higher than most widely used cathodes both in aqueous and non-aqueous electrolytes.Table 1.3 shows a variety of superiron salts and their corresponding capacities. ${ }^{67}$ All of them offer higher specific capacities compared to common materials utilized in primary and secondary alkaline batteries (e.g., $\mathrm{MnO}_{2}$ and $\left.\mathrm{NiOOH}\right)$ and also in common $\mathrm{Li}$ ion cathodes $\left(\mathrm{LiCoO}_{2}, \mathrm{LiMn}_{2} \mathrm{O}_{4}\right.$ and $\left.\mathrm{LiFePO}_{4}\right)$ presented in the same table. Note that $\mathrm{Li}_{2} \mathrm{FeO}_{4}$ offers a theoretical capacity as high as 601 $\mathrm{mAhg}^{-1}$; this is the result of its lower formula mass compared to other super-iron salts. However, when discharging $\mathrm{Li}_{2} \mathrm{FeO}_{4}$ in LiTFB PC:DME electrolyte versus $\mathrm{Li}$ anode, it shows a lower discharge potential compared to that of the other salts. The reason why is 


\begin{tabular}{lll}
\hline Cathode & Charge Storage & Intrinsic Capacity (mAh/g) \\
\hline $\mathrm{Li}_{2} \mathrm{FeO}_{4}$ & $3 \mathrm{e}-$ & 601 \\
$\mathrm{Na}_{2} \mathrm{FeO}_{4}$ & $3 \mathrm{e}-$ & 485 \\
$\mathrm{~K}_{2} \mathrm{FeO}_{4}$ & $3 \mathrm{e}-$ & 406 \\
$\mathrm{Rb}_{2} \mathrm{FeO}_{4}$ & $3 \mathrm{e}-$ & 276 \\
$\mathrm{Cs}_{2} \mathrm{FeO}_{4}$ & $3 \mathrm{e}-$ & 209 \\
$\mathrm{SrFeO}_{4}$ & $3 \mathrm{e}-$ & 388 \\
$\mathrm{BaFeO}_{4}$ & $3 \mathrm{e}-$ & 313 \\
$\mathrm{CaFeO}_{4}$ & $3 \mathrm{e}-$ & 502 \\
$\mathrm{Ag}_{2} \mathrm{FeO}_{4}$ & $5 \mathrm{e}-$ & 399 \\
$\mathrm{MnO}_{2}$ & $2 \mathrm{e}-$ & 150 \\
$\mathrm{NiOOH}$ & $2 \mathrm{e}-$ & 290 \\
$\mathrm{LiCoO}_{2}$ & $1 \mathrm{e}-$ & 272 \\
$\mathrm{LiMn}_{2} \mathrm{O}_{4}$ & $1 \mathrm{e}-$ & 148 \\
$\mathrm{LiFePO}_{4}$ & $1 \mathrm{e}-$ & 170 \\
& &
\end{tabular}

Table 1.3. Comparison of various Fe(VI) salts charge storage and intrinsic capacities of common Li ion battery cathodes. 
that $\mathrm{Li}_{2} \mathrm{FeO}_{4}$ is synthesized with a very low purity $(\sim 20 \%)$ and thus it shows higher polarization when discharged. ${ }^{68}$ Except for $\mathrm{Li}_{2} \mathrm{FeO}_{4}$, the other $\mathrm{Fe}(\mathrm{VI})$ salts have been synthesized by chemical, electrochemical and thermochemical pathways with high purity. A number of different synthesis pathways for various $\mathrm{Fe}(\mathrm{VI})$ salts have been described in depth in the literature. ${ }^{69-74}$

Super-iron cathodes in alkaline media discharge through a three-electron electrochemical reduction reaction: ${ }^{68}$

$$
2 \mathrm{FeO}_{4}{ }^{-}+5 / 2 \mathrm{H}_{2} \mathrm{O}+3 \mathrm{e}^{-} \longrightarrow 1 / 2 \mathrm{Fe}_{2} \mathrm{O}_{3}+5 \mathrm{OH}^{-} \quad \mathrm{E}=0.5-0.65 \mathrm{~V} \text { vs. SHE }
$$

or in organic solvents:

$$
\mathrm{MFeO}_{4}+\mathrm{xLi}++\mathrm{xe}^{-} \longrightarrow \mathrm{Li}_{\mathrm{x}} \mathrm{MFeO}_{4}
$$

where $\mathrm{M}=\mathrm{Na}_{2}, \mathrm{~K}_{2}, \mathrm{Cs}_{2}, \mathrm{Sr}$, Ba, etc. Many of these super-iron salts are viable candidates as cathodes for use in batteries. They all show insolubility in a variety of organic solvents, including carbonate-based solvents mostly used as organic electrolytes in Li ion batteries, and solvents such as propylene carbonate (PC), dimethoxyethane, ethylcarbonate (EC), etc.

Even after addition of lithium salts to these solvents in order to enhance the electrolytic conductivity, they still remain insoluble. $\mathrm{K}_{2} \mathrm{FeO}_{4}$ and $\mathrm{BaFeO}_{4}$ show virtually no dissolution when immersed in dry ACD, PC, DME, etc. ${ }^{74}$ This is in fact an ideal property for a potential cathode, since it minimizes the possibility of dissolution of $\mathrm{Fe}(\mathrm{VI})$, it's migration to the anode and consequent loss of capacity.

Cyclic voltammetry experiments have shown that both $\mathrm{K}_{2} \mathrm{FeO}_{4}$ and $\mathrm{BaFeO}_{4}$ are electrochemically active in non-aqueous solvents. However, a hysteresis is observed between the cathodic and anodic peaks. This hysteresis could be due to the low electrical 
contact in the electrode composite and/or be intrinsic qualities of the super iron salts. ${ }^{75}$ When constructing $\mathrm{Fe}(\mathrm{VI})$ cathodes for use with non-aqueous cells, the presence of increased carbon black content (20\%) enhances the specific capacities (e.g.: for $\mathrm{BaFeO}_{4}$ about $\left.100 \mathrm{mAhg}^{-1}\right)$. Further, it has been illustrated that the charge transfer reactions occur in the known electrochemical range of the electrolytic solutions.

The main challenge facing the application of super-iron salts for use as cathodes in electric vehicle batteries is their low charge transfer reversibility. This arises from the limited conductivity of the ferric oxide discharge product. To tackle this issue, Licht et. al. increased the conductive matrix by electrochemically depositing nano-thin films of superiron salts on a roughened Pt surface. Such a Pt surface has increased surface area and offers an extended conductive matrix that facilitates the charge transfer and sustains the reversibility and capacity up to 100 charge/discharge cycles in both aqueous alkaline and non-aqueous media for films as thick as $571 \mathrm{~nm}$. These nanometer thick layers are also stable at room temperature for up to 7 days, but their stability decreases as the temperature is elevated towards $60 \quad C^{76,77}$

Another approach to increase the reversibility of super-iron salts is to reduce the particle size to the nano domain. In chapter 5 of this dissertation, a new low-heat and gentle mechano-synthesized (ball mill) approach is introduced that reduces the particle size to the nano range. ${ }^{78}$ Reducing the particle size increases the surface area of the active material in the electrode composite, which in turn increases the contact with the electrolyte interface. It also facilitates electron transport and the diffusion rate of the intercalating ions by decreasing the required diffusion lengths. It also better accommodates possible lattice 
expansions due to intercalation and reduces the structural stress induced in the discharge product. ${ }^{79}$ In chapter 6 of this dissertation, the effects of various cations on the reversibility of $\mathrm{MFeO}_{4}$ salts are also studied.

\subsubsection{Characterization of Novel Cathode Materials}

As discussed in previous sections above, there is an extensive search within the scientific community for developing novel materials for use as electrodes for future high capacity batteries. A necessity for designing cathode materials with the desirable attributes is to elucidate the chemical and physical processes at the electrode surface as the battery charges/discharges. The structural and electronic properties of the cathode undergo radical changes during the charge/discharge cycle, and understanding the mechanism of these all important variations and their contribution to the battery performance can provide valuable feedback for design of improved electrode materials.

In recent years, our understanding of the interfacial processes and charge/discharge mechanisms occurring in energy storage devices has notably become deeper. This is all due to the significant progress made in improving the spectroscopic, microscopic and scattering techniques used to study them. The techniques with the capability of studying electrochemical processes in operando or in situ are of crucial importance. Various microscopic and spectroscopic techniques for electrode characterization have been employed both in situ and ex-situ. Here a short review of these techniques and their pros and cons are presented. 
- Transmission Electron Microscopy (TEM) has been used both in-situ and ex-situ for electrode material characterization. TEM combines diffraction, imaging and spectroscopy to provide valuable structural, morphological and compositional information. Techniques that can be performed concurrently with TEM, such as EDS (Energy Dispersive X-ray Spectrometry) and EELS (Electron Energy Loss Spectrometry) have been employed extensively for such purposes ${ }^{80-84}$, especially in characterizing nanoparticles in battery electrodes. To carry out $i n$-situ TEM studies requires complex cell fabrication strategies:

1. In one strategy, a deposition technique such as pulsed laser deposition (PLD) is utilized to carefully deposit multi-layers of transition metal thin films on a substrate to create a "nano-battery". For instance, Gabrisch et. al. formed an all solid-state "nanobattery" utilizing PLD with the following configuration: Au (as current collector)/ $\mathrm{SnO} 2$ (anode) $/ \mathrm{Li}_{3.4} \mathrm{~V}_{0.6} \mathrm{Si}_{0.4} \mathrm{O}_{4}$ (electrolyte)/ $\mathrm{LiCoO}_{2}$ (cathode)/ Pt (current collector). PLD also has the capability of depositing films with controlled crystallographic orientation. ${ }^{85}, 86$ The electrode/electrolyte interface can clearly be imaged by TEM utilizing this technique. However, this strategy specifically needs to utilize solid-state electrolytes. Moreover, utilizing a focused ion beam to fabricate "nano-batteries", researchers should make sure that the electrochemical system is still functioning and not affected by this deposition technique.

2. The second strategy involves using organic liquid electrolytes. This route, although producing a more realistic in-situ battery, faces the challenge of designing an in-situ cell appropriate for introducing an organic electrolyte under high vacuum conditions. For instance, Wang et. al. utilized a vacuum compatible ionic liquid to prevent complex sealing 
of the electrolyte for evaporation inhibition. ${ }^{87,88}$ However, even in this novel approach EELS imaging cannot be utilized since the ionic liquid has a high plasmon signal.

- Solid-state ${ }^{7}$ Li NMR, as well as other nuclei such as ${ }^{31} \mathrm{P}$ and ${ }^{19} \mathrm{~F}$ NMR, can be utilized to identify species on the surface of electrodes. ${ }^{13} \mathrm{C}$ NMR can used to follow the evolution of organic solvents as the charge/discharge cycles progress. ${ }^{7}$ The interpretation of the Li NMR data is quite a challenge, since it is very difficult to resolve the resonances from the different components. This technique has been also utilized to characterize film growth on the surface of electrodes. For instance, ${ }^{7} \mathrm{Li}$ NMR has been utilized to follow and quantify the solid electrolyte interphase (SEI) growth on positive electrodes. ${ }^{89,90}$ However, this technique has mostly been utilized as an ex-situ technique to quantify film growth on electrodes. In the instances that this technique was utilized in-situ, the information provided was mostly focused on the bulk electrochemical mechanisms. As such, there are limitation on following the interfacial electrochemical processes, such as intercalation at the surface of the electrode. Moreover, this technique again requires use of ultrahigh vacuum.

- The scanning probe microscopy (SPM) family of techniques such as Atomic Force Microscopy (AFM) and Scanning Tunneling Microscopy (STM) possess very high spatial resolution which enables atomic scale images of a surface. These techniques can successfully be coupled with electrochemical methods and utilized in-situ to follow interfacial phenomena. However, again there are many challenges with the application of this family of techniques to batteries, especially Li-ion batteries. The in-situ cell will need to be exposed to the probe while scanning (rastering over) the electrode surface. This means that the SPM system should be set in a oxygen free and water free atmosphere. 
Therefore, it is recommended that the SPM unit sit in an argon glove box with water and oxygen content of less than $1 \mathrm{ppm}$. Further, the SPM unit is extremely sensitive to vibrations. Hence, the glove box should also be vibration proof. Another challenge with employing these techniques is that they cannot be utilized to probe electrode composites. ${ }^{91}$

As mentioned previously, most cathodes are comprised of active material, a conductive matrix such as carbon black to increase electron conductivity and a binder; i.e. they are composed of composites. These electrodes have very high surface roughness, and therefore small variations incurred on the electrode surface due to charge/discharge and intercalation process will not be detected when using these composites. Again as in TEM techniques, a deposition technique or sputtering technique should be used to prepare thin smooth film electrodes.

- $\underline{\text { X-ray diffraction }(\mathrm{XRD})}$ has provided vital information regarding long-range structural changes caused by intercalation processes. However, it requires relatively long range order in the material and following surface phenomena is not possible with this technique.

- X-ray absorption spectroscopy (XAS) has in contrast provided insight into both the electronic and structural changes of cathode materials. Further, this technique can easily be used for in-situ studies as the probe and the signal are both x-rays, enabling in-situ experiments to be conducted with relative ease. Further, since ultrahigh vacuum is not needed as with electron spectroscopies, in-situ studies can easily be conducted on Li-ion batteries utilizing a well-sealed cell such as pouch cells.

XAS has several other properties that make it ideal for characterization of high capacity cathode materials: 
- It is an element specific technique that is sensitive to dilute samples (as low as 10$100 \mathrm{ppm})$.

- It is a short range order technique that can be utilized for study of amorphous materials and small particles such as nanoparticles. Chapter 4 of this dissertation reports results of novel nanoparticle super iron salts as cathode materials utilizing XAS.

- It can simultaneously provide electronic (such as oxidation state and site symmetry) and structural information.

\subsubsection{Previous Characterization of Super-Iron Cathodes}

Super-iron salts in both charged and discharged states have been characterized qualitatively and quantitatively by a variety of electrochemical and spectroscopic methods.

- FTIR spectroscopy has been utilized to quantitatively follow the amounts of $\mathrm{Fe}(\mathrm{VI})$ present in electrode composites. ${ }^{91,92}$ An inert standard, such as $\mathrm{BaSO}_{4}$ with a clear IR peak well resolved from $\mathrm{Fe}(\mathrm{VI})$, should be added to the $\mathrm{KBr}$ palette when conducting quantitative measurements to compensate for the low weight percent $(\sim 1 \%)$ of $\mathrm{Fe}(\mathrm{VI})$ in the palette. Various $\mathrm{Fe}(\mathrm{VI})$ salts, as well as the discharge product (Fe(III)) can be identified and measured quantitatively with this technique, yet electronic and structural information and insight into the charge/discharge mechanism cannot be studied using this technique.

- Mossbauer spectroscopy. Both in-situ and ex-situ Mossbauer spectroscopy studies on $\mathrm{Fe}(\mathrm{VI})$ were conducted using a constant acceleration Mossbauer drive and a $50 \mathrm{mCi}^{57}$ Co:Rh source. A thin $\alpha$-iron film was utilized to calibrate the velocity and as a isomer shift reference. Both quantitative and qualitative analyses were performed. However, this technique could not provide conclusive insight into the nature of the mostly amorphous 
discharge product. It was speculated that the discharge product is a polymorph of $\mathrm{FeOOH}$ and $\mathrm{Fe}_{2} \mathrm{O}_{3}$, but further understanding of the electronic and structural changes of the cathode as a function of discharge/charge progress was not plausible. ${ }^{93,94,95}$

- $\underline{\text { XRD }}$ is another technique utilized to study these salts. ${ }^{96}$ It has been used for both qualitative and quantitative measurements. However, one cannot follow the electronic variations as the charge/discharge progresses.

As mentioned previously, there is a significant need for utilizing a short range element specific technique such as XAS to elucidate the nature of the non-conductive and amorphous Fe(III) discharge product and elucidate the mechanism of the charge/discharge process in these cathodes.

\subsection{Summary:}

In this dissertation, XAS combined with electrochemical and theoretical techniques, will be utilized to characterize the electrochemical processes occurring in both fuel cells and batteries. The motivation for this research is two fold:

1. To understand the surface reaction mechanisms and adsorbate binding sites of reactants and/or poisons on the catalyst surface in fuel cells. Chapter 3 of this dissertation is focused on the competitive adsorption of halides on Pt utilizing in-situ XAS. Chapter 4 is a space-resolved study to gain knowledge of the adsorbate distribution on both the anode and cathode in a DMFC utilizing in-operando XAS. This provides insight into the effects of non-uniform adsorbate distribution on the overall performance of the fuel cell. 
2. To understand the multi-electron charge transfer mechanism in super-iron salts. Chapters 5 and 6 of this dissertation focus on an investigation of the reversible charge transfer in these high capacity cathodes. As discussed above, these cathodes suffer from poor reversibility. In chapter 5, a new mechano-synthesis approach is taken to synthesize nano size super iron particles. Ex-situ XAS is utilized to characterize the amorphous discharge product and understand the mechanism behind the higher capacity (and possible reversibility) of the nanoparticles. Chapter 6 reports an investigation of the charge transfer mechanism in two different super iron salts $\left(\mathrm{K}_{2} \mathrm{FeO}_{4}\right.$ and $\left.\mathrm{BaFeO}_{4}\right)$ and utilizing XAS to understand the variations in the charge transfer rates and reversibility as a function of the cation in these salt. 


\subsection{References}

(1) Davis, S. C.; Diegel, S. W.; Boundy, R. G. In Transportation Energy Data Book: Edition 31; 2010.

(2) Thomas, E. S. Fuel Cell and Battery Electric Vehiceles Compared. Intl. J. Hydrogen Energy 2009, 34, 6005-6020.

(3) Thomas, E. S. "How green are electric vehicles?". Intl. J. Hydrogen Energy 2012, 37, 6053.

(4) Larminie, J.; Dicks, A. In Fuel Cell Systems Explained, Second Edition; John Wiley \& Sons Ltd: West Sussex, England, 2003.

(5) Behling, N. H. In Fuel Cells: Current Technology Challenges and Future Research Needs, First Edition; Elsevier: Amsterdam, The Netherlands, 2012.

(6) EG\&G Services Parsons, Inc. Science Applications International Corporation In Fuel Cell Handbook, Fifth Edition; U.S. Department of Energy, Office of Fossil Energy, National Energy Technology Laboratory: Morgantown, West Virginia, 2000.

(7) Hogarth, M. P.; Ralph, T. R. Catalysis for Low Temperature Fuel Cells. Platinum Metals Review 2002, 46, 146.

(8) Shukla, A. K.; Raman, R. K. Methanol-Resistant Oxygen-Reduction Catalysts for Direct Methanol Fuel Cells. Annual Rev. Mater. Res. 2003, 33, 155.

(9) Aasberg-Petersen, K.; Nielsen, C. S.; Jorgensen, S. L. Membrane Reforming for Hydrogen. Catal. Today 1998, 46, 193.

(10) Oh, S. H.; Sinkevitch, R. M. Carbon Monoxide Removal from HydrogenRich Fuel Cell Feedstreams by Selective Catalytic Oxidation. J. Catal. 1993, 142, 254.

(11) Gasteiger, H. A.; Markovic, N.; Ross, P. N.; Cairns, E. J. Methanol Oxidation on Well-Characterized Platinum-Ruthenium Bulk Alloys. J. Phys. Chem. 1993, 97, 12020.

(12) Roth, C.; Benker, N.; Buhrmester, T.; Mazurek, M.; Loster, M.; Fuess, H.; Koningsberger, D. C.; Ramaker, D. E. Determination of $\mathrm{O}[\mathrm{H}]$ and CO Coverage and 
Adsorption Sites on PtRu Electrodes in an Operating PEM Fuel Cell. J. Am. Chem. Soc. 2005, 127, 14607.

(13) Kua, J.; Goddard, W. A., III. Oxidation of Methanol on 2nd and 3rd Row Group VIII Transition Metals (Pt, Ir, Os, Pd, Rh, and Ru): Application to Direct Methanol Fuel Cells. J. Am. Chem. Soc. 1998, 121, 10928.

(14) Mukerjee, S.; Urian, R. C.; Lee, S. J.; Ticianelli, E. A.; McBreen, J. Electrocatalysis of CO Tolerance by Carbon-Supported PtMo Electrocatalysts in PEMFCs. J. Electrochem. Soc. 2004, 151, A1094.

(15) Mukerjee, S.; Urian, R. C. Bifunctionality in Pt alloy nanocluster electrocatalysts for enhanced methanol oxidation and $\mathrm{CO}$ tolerance in PEM fuel cells: electrochemical and in situ synchrotron spectroscopy. Electrochim. Acta 2002, $47,3219$.

(16) Urian, R. C.; Gulla, A. F.; Mukerjee, S. Electrocatalysis of reformate tolerance in proton exchange membranes fuel cells: Part I.J. Electroanal. Chem. 2003, 554-555, 307.

(17) Grgur, B. N.; Zhuang, G.; Markovic, N. M.; Ross, P. N., Jr. Electrooxidation of $\mathrm{H}_{2} / \mathrm{CO}$ Mixtures on a Well-Characterized $\mathrm{Pt}_{75} \mathrm{Mo}_{25}$ Alloy Surface. J. Phys. Chem. B 1997, 101, 3910.

(18) Lu, C.; Rice, C.; Masel, R. I.; Babu, P. K.; Waszczuk, P.; Kim, H. S.; Oldfield, E.; Wieckowski, A. UHV, Electrochemical NMR, and Electrochemical Studies of Platinum/Ruthenium Fuel Cell Catalysts. J. Phys. Chem. B 2002, 106, 9581.

(19) Koper, M. T. M.; Shubina, T. E.; van Santen, R. A. Periodic Density Functional Study of $\mathrm{CO}$ and $\mathrm{OH}$ Adsorption on $\mathrm{Pt}-\mathrm{Ru}$ Alloy Surfaces: Implications for CO Tolerant Fuel Cell Catalysts. J. Phys. Chem. B 2002, 106, 686.

(20) Skelton, D. C.; Tobin, R. G.; Lambert, D. K.; DiMaggio, C. L.; Fisher, G. B. Oxidation of CO on Gold-Covered Pt(335). J. Phys. Chem. B 1999, 103, 964.

(21) Teliska, M.; O’Grady, W. E.; Ramaker, D. E. Determination of O and OH Adsorption Sites and Coverage in Situ on $\mathrm{Pt}$ Electrodes from $\mathrm{Pt} \mathrm{L}_{23} \mathrm{X}$-ray Absorption Spectroscopy. J. Phys. Chem. B 2005, 109, 8076.

(22) Scott, F. J.; Mukerjee, S.; Ramaker, D. E. CO Coverage/Oxidation Correlated with PtRu Electrocatalyst Particle Morphology in $0.3 \mathrm{M}$ Methanol by In Situ XAS. J. Electrochem. Soc.2007, 154, A396. 
(23) Paulus, U. A.; Schmidt, T. J.; Gasteiger, H. A. In Posions for the O2 Reduction Reaction; Vielstich, W., Gasteiger, H. A., Lamm, A. and Yokokawa, H., Eds.; Handbook of Fuel Cells - Fundamentals, Technology and Applications. John Wiley \& Sons, Ltd.: 2010.

(24) Markovic, N. M.; Schmidt, T. J.; Stamenkovic, V.; Ross, P. N. Oxygen Reduction Reaction on Pt and Pt Bimetallic Surfaces: A Selective Review. Fuel Cells 2001, 1, 105.

(25) Schmidt, T. J.; Paulus, U. A.; Gasteiger, H. A.; Behm, R. J. The Oxygen Reduction Reaction on Pt/Carbon Fuel Cell Catalysts in the Presence of Chloride Anions. J. Electroanal. Chem. 2001, 508, 41.

(26) Zhang, L.; Mukerjee, S. Investigation of Durability Issues of Selected Nonfluorinated Proton Exchange Membranes for Fuel Cell Application. $J$. Electrochem. Soc. 2006, 153, A1062.

(27) Yoshitake, M.; Yanagisawa, E.; Terada, I.; Shimoda, H.; Hommura, S.; Ishisaki, T.; Kunisa, Y. In B"uchi, F. N., Scherer G.G. and Wokaun, A., Eds.; '1st European Fuel Cell Forum - Proceedings'; Forum Oberrohrdorf: 2001, 109.

(28) Stamenkovic, V.; Markovic, N. M. Oxygen Reduction and Hydrogen Oxidation Reactions on $\operatorname{Pt}(111)$ and $\operatorname{Pt}(100)$ in Solutions Containing Copper Ions. Langmuir 2001, 17, 2388.

(29) Balbuena, P. B.; Altomare, D.; Vadlamani, N.; Bingi, S.; Agapito, L. A.; Seminario, J. M. Adsorption of $\mathrm{O}, \mathrm{OH}$, and $\mathrm{H}_{2} \mathrm{O}$ on Pt-Based Bimetallic Clusters Alloyed with Co, Cr, and Ni. J. Phys. Chem. A 2004, 108, 6378.

(30) Teliska, M.; Murthi, V. S.; Mukerjee, S.; Ramaker, D. E. Correlation of Water Activation, Surface Properties, and Oxygen Reduction Reactivity of SupportedPt-M/C Bimetallic Electrocatalysts Using XAS.J. Electrochem. Soc. 2005, 152, A1259.

(31) Teliska, M.; O'Grady, W. E.; Ramaker, D. E. Determination of O and OH Adsorption Sites and Coverage in Situ on Pt Electrodes from Pt L23 X-ray Absorption Spectroscopy. J. Phys. Chem. B2005, 109, 8076.

(32) Teliska, M. Determination of $\mathrm{H}, \mathrm{O}$ and $\mathrm{OH}$ Chemisorption Sites on Platinum and Platinum-based Alloy Electrodes in an Electrochemical Cell using in situ X-ray Absorption Spectroscopy, The George Washington University, Washington, D.C., 2004. 
(33) Ye, Q.; Zhao, H.; Yang, J.; Prabhuran, J. Electrochemical Reactions in a DMFC under Open-Circuit Conditions. J. Electrochem. Solid-State Lett. 2005, 8, A52.

(34) Sauer, D. U.; Sanders, T.; Fricke, B.; Baumhofer, T.; Wipperman, K.; Kulikovsky, A. A.; Schmitz, H.; Mergel, J. Measurement of the current distribution in a direct methanol fuel cell-Confirmation of parallel galvanic and electrolytic operation within one cell. J. Power Sources 2008, 176, 477.

(35) Dixon, D.; Habereder, A.; Farmand, M.; Kaserer, S.; Roth, C.; Ramaker, D. E. Space Resolved, in Operando X-ray Absorption Spectroscopy: Investigations on Both the Anode and Cathode in a Direct Methanol Fuel Cell. J. Phys. Chem. C 2012, 116, 7587.

(36) Nashner, M. S.; Frenkel, A. I.; Adler, D. L.; Shapley, J. R.; Nuzzo, R. G. Structural Characterization of Carbon-Supported Platinum-Ruthenium Nanoparticles from the Molecular Cluster Precursor $\mathrm{PtRu}_{5} \mathrm{C}(\mathrm{CO})_{16}$. J. Am. Chem. Soc. 1997, 119, 7760.

(37) Nashner, M. S.; Frenkel, A. I.; Somerville, D.; Hills, C. W.; Shapley, J. R.; Nuzzo, R. G. Core Shell Inversion during Nucleation and Growth of Bimetallic Pt/Ru Nanoparticles. J. Am. Chem. Soc. 1998, 120, 8093.

(38) http://www.nissanusa.com/leaf-electric-car/battery.

(39) http://www.rolandberger.com/media/pdf/Roland_Berger_Li_Ion_Batteries Bubble Bursts 20121019.pdf.

(40) Whittingham, M. S. Lithium Batteries and Cathode Materials. Chem. Rev. 2004, 104, 4271.

(41) Ellis, B. L.; Lee, K. T.; Nazar, L. F. Positive Electrode Materials for Li-Ion and Li-Batteries. Chem. Mater. 2010, 22, 691.

(42) Cheng , F.; Liang, J.; Tao, Z.; Chen, J. Functional Materials for Rechargeable Batteries. Adv. Mater. 2011, 23, 1695.

(43) .Xiao, J.; Chernova , N. A.; Whittingham, M. S. Influence of Manganese Content on the Performance of $\mathrm{LiNi}_{0.9-y} \mathrm{Mn}_{y} \mathrm{Co}_{0.1} \mathrm{O}_{2}(0.45 \leq y \leq 0.60)$ as a Cathode Material for Li-Ion Batteries. Chem. Mater. 2010, 22, 1180.

(44) Luo, W. B.; Dahn, J. R. Comparative study of $\mathrm{Li}\left[\mathrm{Co}_{1-z} \mathrm{Al}_{z}\right] \mathrm{O}_{2}$ prepared by solid-state and co-precipitation methods. Electrochim. Acta 2009, 54, 4655. 
(45) Jang, Y. I.; Huang, B. Y.; Wang, H. F.; Sadoway, D. R.; Ceder, G.; Chiang, Y. M.; Liu, H.; Tamura, H. J. $\mathrm{LiAl}_{y} \mathrm{Co}_{1}-y \mathrm{O}_{2}(\mathrm{R} 3 m)$ Intercalation Cathode for Rechargeable Lithium Batteries. J. Electrochem. Soc. 1999, 146, 862.

(46) Winter, M.; Besenhard, J. O.; Spahr, M. E.; Novák, P. Insertion Electrode Materials for Rechargeable Lithium Batteries. Adv. Mater. 1998, 10, 725.

(47) Ariyoshi, K.; Iwata, E.; Kuniyoshi, M.; Wakabayashi, H.; Ohzuku, T. Lithium Aluminum Manganese Oxide Having Spinel-Framework Structure for Long-Life Lithium-Ion Batteries. Electrochem. Solid State Lett. 2006, 9, A557.

(48) Padhi , A. K.; Nanjundaswamy, K. S.; Goodenough, K. S. Phospho-olivines as Positive-Electrode Materials for Rechargeable Lithium Batteries. J. Electrochem. Soc. 1997, 144, 1188.

(49) A.Churikov, V.; Ivanishchev , A. V.; Ivanishcheva, I. A.; Sycheva , V. O.; Khasanova, N. R.; Antipov, E. V. Determination of lithium diffusion coefficient in $\mathrm{LiFePO}_{4}$ electrode by galvanostatic and potentiostatic intermittent titration techniques. Electrochim. Acta 2010, 55, 2939.

(50) Amin, R.; Maier, J.; Balaya, P.; Chen, D. P.; Lin, C. T. Ionic and electronic transport in single crystallinem $\mathrm{LiFePO}_{4}$ grown by optical floating zone technique. Solid State Ionics 2008, 179, 1683.

(51) Prosini, P. P.; Lisi, M.; Zane, D.; Pasquali, M. Determination of the chemical diffusion coefficient of lithium in $\mathrm{LiFePO}_{4}$. Solid State Ionics 2002, 148, 45 .

(52) Huang, H.; Yin, S. -.; Nazar, L. F. Approaching Theoretical Capacity of $\mathrm{LiFePO}_{4}$ at Room Temperature at High Rates. Electrochem. Solid State Lett. 2001, 4, A170.

(53) Yang, S.; Song, Y.; Ngala, K.; Zavalij, P. Y.; Whittingham, M. S. Performance of $\mathrm{LiFePO}_{4}$ as lithium battery cathode and comparison with manganese and vanadium oxides. J. Power Sources 2003, 119, 239.

(54) Wu, X. L.; Jiang, L. Y.; Cao, F. F.; Guo, Y. G.; Wan, L. J. $\mathrm{LiFePO}_{4}$ Nanoparticles Embedded in a Nanoporous Carbon Matrix: Superior Cathode Material for Electrochemical Energy-Storage Devices. Adv. Mater. 2009, $21,2710$.

(55) Wang, Y.; Wang, Y.; Hosono, E.; Wang, K.; Zhou, H. The Design of a $\mathrm{LiFePO}_{4} /$ Carbon Nanocomposite With a Core-Shell Structure and Its Synthesis by 
an In Situ Polymerization Restriction Method. Angew Chem. Intl. Ed. 2008, 47, 7461 .

(56) Shiratsuchi, T.; Okada, S.; Doi, T.; Yamaki, J. I. Cathodic performance of LiMn1-xMxPO4 $(\mathrm{M}=\mathrm{Ti}, \mathrm{Mg}, \mathrm{Zr})$ annealed in an inert atmosphere. Electrochim. Acta 2009, 54, 3145.

(57) Kim, S. W.; Kim, J.; Gwon, H.; Kang, K. Phase stability study of Li1-xMnPO4 $(0 \leq \mathrm{x} \leq 1)$ cathode for Li rechargeable battery. Electrochimi Acta 2009, 156, A635.

(58) Murugan, A. V.; Muraliganth, T.; Manthiram, A. One-pot microwavehydrothermal synthesis and characterization of carbon-coated LiMPO4(M= Mn, Fe, and Co) cathodes. J. Electrochem. Soc. 2009, 156, A79.

(59) Barker, J.; Saidi, M. Y.; Swoyer, J. L. Electrochemical Insertion Properties of the Novel Lithium Vanadium Fluorophosphate, $\mathrm{LiVPO}_{4}$ F. J. Electrochem. Soc. 2003, 150, A1394.

(60) Nyten, A.; Abouimrane, A.; Armand, M.; Gustafsson, T.; Thomas, J. O. Electrochemical performance of $\mathrm{Li}_{2} \mathrm{FeSiO}_{4}$ as a new Li-battery cathode material. Electrochem. Comm. 2005, 7, 156.

(61) Barpanda, P.; Ati, M.; Melot, B. C.; Rousse, G.; Chotard, J. N.; Doublet, M. L.; Sougrati, M. T.; Corr, S. A.; Jumas, J. C.; Tarascon, J. M. A 3.90 V ironbased fluorosulphate material for lithium-ion batteries crystallizing in the triplite structure. Nat. Mater. 2011, 10, 772.

(62) Gao, X. P.; Yang, H. X. Multi-electron reaction materials for high energy density batteries. Energy and Environmental Science 2010, 3, 174.

(63) Badway, F.; Cosandey, F.; Pereira, N.; Amatucci, G. G. Carbon Metal Fluoride Nanocomposites: High-Capacity Reversible Metal Fluoride Conversion Materials as Rechargeable Positive Electrodes for Li Batteries. J. Electrochem. Soc. 2003, 150, A1318.

(64) Li, H.; Richter, G.; J. Maier, J. Reversible Formation and Decomposition of LiF Clusters Using Transition Metal Fluorides as Precursors and Their Application in Rechargeable Li Batteries. Adv. Mater.2003, 15, 736.

(65) Ma, Y.; Garofalini, S. H. Atomistic Insights into the Conversion Reaction in Iron Fluoride: A Dynamically Adaptive Force Field Approach. J. Am. Chem. Soc. 2012, 134, 8205 . 
(66) Licht, S.; Wang, B.; Ghosh, S. Energetic Iron(VI) Chemistry: The SuperIron Battery. Science 1999, 13, 1039.

(67) $\mathrm{Yu}, \mathrm{X}$.; Licht, S. Recent advances in synthesis and analysis of $\mathrm{Fe}(\mathrm{VI})$ cathodes: solution phase and solid-state $\mathrm{Fe}(\mathrm{VI})$ syntheses, reversible thin-film $\mathrm{Fe}(\mathrm{VI})$ synthesis, coating-stabilized $\mathrm{Fe}(\mathrm{VI})$ synthesis, and $\mathrm{Fe}(\mathrm{VI})$ analytical methodologies. J. Solid State Electrochem. 2008, 12, 1523.

(68) Licht, S. In Super-Iron Batteries Garche, J., Dyer, C., Moseley, P., Ogumi, Z., Rand, D. and Scrosati, B., Eds.; Encyclopedia of Electrochemical Power Sources; Elsevier: Amsterdam, The Netherlands, 2009; Vol. 4, pp 262.

(69) Licht, S.; Naschitz, V.; Ghosh, S.; Liu, B.; Halperine, N.; Halperin, L.; Rozen, D. Chemical synthesis of battery grade super-iron barium and potassium Fe(VI) ferrate compounds. J. Power Sources 2001, 99, 7.

(70) Walz, K.; Suyama, A.; Suyama, W.; Sene, J.; Zeltner, W.; Armacanqui, E.; Roszkowski, A.; Anderson, M. Characterization and performance of high power iron(VI) ferrate batteries. J. Power Sources 2004, 134, 318.

(71) Licht, S.; Yu, X.; Qu, D. A novel alkaline redox couple: chemistry of the $\mathrm{Fe}^{6+} / \mathrm{B}^{2-}$ super-iron boride battery. Chem. Commun. 2007, 26, 2753.

(72) Li, C.; Li, X.; Graham, N. A study of the preparation and reactivity of potassium ferrate. Chemosphere 2005, 61, 537.

(73) Licht, S.; Wang, B. Nonaqueous Phase Fe ( VI ） Electrochemical Storage and Discharge of Super-Iron/Lithium Primary Batteries. Electrochem. Solid State Lett. 2000, 3, 209.

(74) Licht, S.; Naschitz, V.; Rozen, D.; Halperin, N. Cathodic Charge Transfer and Analysis of $\mathrm{Cs}_{2} \mathrm{FeO}_{4}, \quad \mathrm{~K}_{2} \mathrm{FeO}_{4}$, and Mixed Alkali Fe(VI) Ferrate Super-irons. J. Electrochem Soc 2004, 151, A1147.

(75) Koltypin, M.; Licht, S.; Nowik, I.; Vered, R. T.; Levi, E.; Gofer, Y.; Aurbacha, D. Study of Various ("Super Iron") MFeO4 Compounds in Li Salt Solutions as Potential Cathode Materials for Li Batteries. J. Electrochem. Soc. 2006, 153, A 32 .

(76) Licht, S.; Wang, Y.; Gourdin, G. Enhancement of Reversible Nonaqueous Fe(III/VI) Cathodic Charge Transfer. J. of Phys. Chem. C 2009, 113, 9884. 
(77) Licht, S.; De Alwis, C. Conductive-Matrix-Mediated Alkaline Fe(III/VI) Charge Transfer: Three-Electron Storage,Reversible Super-Iron Thin Film Cathodes . J. Phys. Chem. B 2006, 110, 12394.

(78) Farmand, M.; Jiang, D.; Wang, B.; Ghosh, S.; Ramaker, D. E.; Stuart Licht, S. Super-iron Nanoparticles with Facile Cathodic Charge Transfer. Electrochem. Commun. 2011, 13, 909.

(79) Bruce, P. G.; Scrosati, B.; Tarascon, J. M. Nanomaterials for Rechargeable Lithium Batteries. Angew Chem. Intl Ed. 2008, 47, 2930.

(80) Yoshida, J.; Stark, M.; Holzbock, J.; Husing, N.; Nakanishi, S.; Iba, H.; Abe, H.; Naito, M. Analysis of the size effect of $\mathrm{LiMnPO}_{4}$ particles on the battery properties by using STEM-EELS . J. Power Sources 2013, 226, 122.

(81) Shiraishi, Y.; Nakai, I.; Kimoto, K.; Matsui, Y. EELS analysis of electrochemically deintercalated $\mathrm{Li}_{1-x} \mathrm{Mn}_{2} \mathrm{O}_{4}$ and substituted spinels $\mathrm{LiMn}_{1.6} \mathrm{M}_{0.4} \mathrm{O}_{4}(\mathrm{M}=\mathrm{Co}, \mathrm{Cr}, \mathrm{Ni})$. J. Power Sources 2001, 97-98, 461.

(82) Boulineau, A.; Simonin, L.; Colin, J.; Canévet , E.; Daniel, L.; Patoux, S. Evolutions of $\mathrm{Li}_{1.2} \mathrm{Mn}_{0.61} \mathrm{Ni}_{0.18} \mathrm{Mg}_{0.01} \mathrm{O}_{2}$ during the Initial Charge/Discharge Cycle Studied by Advanced Electron Microscopy.Chem. Mater. 2012, 24, 3558.

(83) Okubo, M.; Kim, J.; Kudo, T.; Zhou, H.; Honma, I. Anisotropic Surface Effect on Electronic Structures and Electrochemical Properties of $\mathrm{LiCoO}_{2}$. J. Phys. Chem. C 2009, 113, 15337.

(84) Danil de Namor, A.; Tanco, M.; Salomon, M.; Ng, J. Thermodynamic, Structural, and Conductance Studies of Lithium Coronand Electrolytes Relevant to Lithium Battery Technology. J. Phys. Chem. 1994, 98, 11796.

(85) Gabrisch, H.; Yazami, R.; Fultz, B. A transmission electron microscopy study of cycled $\mathrm{LiCoO}_{2}$. J. Power Sources 2003, 119, 674.

(86) Kuwataa, N.; Kawamuraa, J.; Toribamia, K.; Hattoria, T.; Satab, N. Thinfilm lithium-ion battery with amorphous solid electrolyte fabricated by pulsed laser deposition. Electrochem. Commun. 2004, 6, 417.

(87) Wang, C. M.; Xu, W.; Liu, J.; Choi, W.; Arey, B.; Saraf, L. V.; Zhang, J. G.; Yang, Z. G.; Thevuthasan, S.; Baer, D. R.; Salmon, N. In situ transmission electron microscopy and spectroscopy studies of interfaces in $\mathrm{Li}$ ion batteries: Challenges and opportunities. J. Mater. Res. 2010, 25, 1541. 
(88) Wang, C. M.; Xu, W.; Liu, J.; Ji-Zhang, G.; Saraf, L. V.; Arey, B. W.; Choi, D.; Yang, Z. G.; Xiao, J.; Thevuthasan, S.; Baer, R. D. In Situ Transmission Electron Microscopy Observation of Microstructure and Phase Evolution in a $\mathrm{SnO}_{2}$ Nanowire during Lithium Intercalation. Nano Lett. 2011, 11, 1874.

(89) Smart, M. C.; Ratnakumar, B. V.; Surampudi, S.; Wang, Y.; Zhang, X.; Greenbaum, S. G.; Hightower, A.; Ahn, C. C.; Fultz, B. Irreversible Capacities of Graphite in Low-Temperature Electrolytes for Lithium-Ion Batteries. $J$. Electrochem. Soc. 1999, 146, 3963.

(90) Meyera, B. M.; Leifera, N.; Sakamotob, S.; Greenbauma, S. G.; Grey, C. P. High Field Multinuclear NMR Investigation of the SEI Layer in Lithium Rechargeable Batteries. Electrochem. Solid State Lett. 2005, 8, A145.

(91) Inaba, M.; Jeong, S. K.; Ogumi, Z. In Situ Scanning Probe Microscopy of Interfacial Phenomena in Batteries. Intreface 2011, $20,55$.

(92) Licht, S.; Naschitz, V.; Lin, L.; Chen, J.; Ghosh , S.; Liu, B. Analysis of ferrate(VI) compounds and super-iron Fe(VI) battery cathodes: FTIR, ICP, titrimetric, XRD, UV/VIS, and electrochemical characterization. $J$ of Power Sources 2001, 101, 167.

(93) Nowika, I.; Herbera, R. H.; Koltypinb, M.; Aurbachb, D.; Licht, S. Mossbauer spectroscopic studies of the disintegration of hexavalentiron compounds (BaFeO4 and K2FeO4). J. Phys. Chem. Solids 2005, 66, 1307.

(94) Ayersa, K. E.; White, N. C. Characterization of Iron,,VI... Compounds and Their Discharge Products in Strongly Alkaline Electrolyte. J. Electrochem Soc. 2005, 152, A467.

(95) Ghosh, S.; Wen, W.; Urian, R. C.; Heath, C.; Srinivasamurthi, V.; Reiff, W. M.; Mukerjee, S.; Naschitz, V.; Licht, S. Reversible Behavior of K2Fe,,VI...O4 in Aqueous Media: In Situ 57Fe Mo"ssbauer and Synchrotron X-Ray Spectroscopy Studies. Electrochem. Solid State Lett. 2003, 6, A260.

(96) Licht, S.; Nashcitz, V.; Wang., B.; Rapid Chemical Synthesis of the Barium Ferrate Super-iron Fe(VI) Compound, $\mathrm{BaFeO}_{4}$, J. Power Sources 2002, 109, 67.

(97) Wroblowa, H.S.; Pan, Y.C.; Razumney, G..; Electroreduction of Oxygen: A New Mechanistic Criterion, J. of Electroanal. Chem. 1976, 69, 195. 
(98) Roth, C.; Ramaker, D.E.; In XAS Investigations of PEM Fuel Cells, C.G. Vayenas, C.G.; Ed.; Interfacial Phenomena in Electrocatalysis, Modern Aspects of Electrochemistry, Springer Science. 


\section{Chapter 2: Introduction to X-ray Absorption Spectroscopy: Experimental and Theoretical Methods}

\subsection{Introduction}

X-ray absorption spectroscopy (XAS) is an ideal technique for studying the electrochemical processes occurring on an electrode surface in energy conversion and storage devices. XAS is well suited for in situ spectro-electrochemical studies because both the incoming probe and outgoing signal are $\mathrm{X}$-rays that can penetrate the sample. It is an element specific technique that can inform both the electronic and structural properties of a particular element in a complex environment. Since XAS is fundamentally a short range photoelectron scattering technique, it is ideal for studying amorphous and nano materials. This chapter will discuss the fundamentals of XAS, as well as the experimental and data analysis methods utilized throughout this dissertation.

\subsection{Theory of X-ray Absorption Spectroscopy}

In the energy range of interest for XAS $(\mathrm{E}<35 \mathrm{keV})$, the main interactions of light and matter are limited to Rayleigh (elastic) scattering, Compton (inelastic) scattering and the photoelectric effect. The photoelectric effect is the only process of importance to XAS; the other two processes will only contribute to the absorption background. When a beam of X-ray photons hits an atom, the atom acquires all the energy of this beam. This energy excites core level electrons into higher unoccupied electron orbitals or into the continuum, creating "photoelectrons". The excitation of electrons and ejection of photoelectrons 47 
creates core holes in the atom. Following the creation of photoelectrons, and within a femto second of the excitation process, the relaxation process occurs which involves the transition of an electron from valence levels back to the unoccupied core level. The transition of the valence electrons to fill out the core hole occurs along with release of energy as fluorescence emission, Auger electron production or secondary electron/photon production. ${ }^{1}$

The X-ray beam irradiating the material under study, which is the source of the excitation process, loses its incident intensity $\left(\mathrm{I}_{0}\right)$ by an amount which is dependent on the characteristics of the material under study. For a sample of a specific thickness (here x), the decrease in initial beam intensity is derived through the following Eq.:

$$
d I=-\mu(E) \cdot I \cdot d x
$$

Here $\mu(E)$, the linear absorption coefficient, is a function of the photon energy ${ }^{2}$ :

$$
\mu(E) \sim \rho Z^{4} / A E^{3}
$$

where $\mathrm{E}$ is the $\mathrm{x}$-ray energy, $\mathrm{Z}$ is the atomic number, $\rho$ is the electron density and $\mathrm{A}$ is the atomic mass. Integrating Eq. 1 over the total thickness of the sample (x) will the yield Lambert's law:

$$
I=I_{o} . e^{-\mu(E) x}
$$

As can be deduced from Eq. 3, the thickness of the sample has an exponential effect on the intensity of the transmitted beam; increasing the thickness of sample by one absorption length unit will decrease the transmitted X-ray beam's energy by $\sim 63 \%$.

Eq. 2 indicates the dependency of the absorption coefficient $(\mu)$ on the elemental properties of the sample. The higher the atomic mass, the larger $\mu$ will be. When the 
incident x-ray photon energy is large enough to cause the formation of a core-hole, the linear absorption coefficient will show a significant increase. These sharp increases in the absorption coefficient are called "absorption edges". Each element possesses a set of specified absorption edges, which correlate with the binding energies of its electrons. Figure 2.1 shows the K-edge and L-edge energies of various elements as a function of their atomic number $(Z)$. As can be observed, the energy of a specific edge increases with the increase of atomic number. The edges are named based on the subshell from which the core level excitations occur; Table 2.1 illustrates the X-ray absorption edges and core level excitations.

Based on Fermi's Golden Rule the linear absorption coefficient is proportional to the probability of absorption of a photon:

$$
\mu(E)=\frac{8 \pi e^{2} \omega^{3} n}{h c^{3}}\left|\left\langle\psi_{f}|\boldsymbol{E} \cdot \boldsymbol{r}| \psi_{i}\right\rangle\right|^{2} \cdot \delta\left(E_{f}-E_{i}-h v\right)
$$

Here:

e: electron charge

$\omega$ : angular frequency of incoming photon

c: speed of light

$\psi_{\mathrm{f}}$ : final state wave function of the system including the bound electrons, core hole and excited photoelectron

$\psi_{\mathrm{i}}$ : initial state wave function of the system, the bound electrons

E: electric field polarization vector

r: position vector of the photon with respect to absorber

$\delta$ : density of states at the final energy 


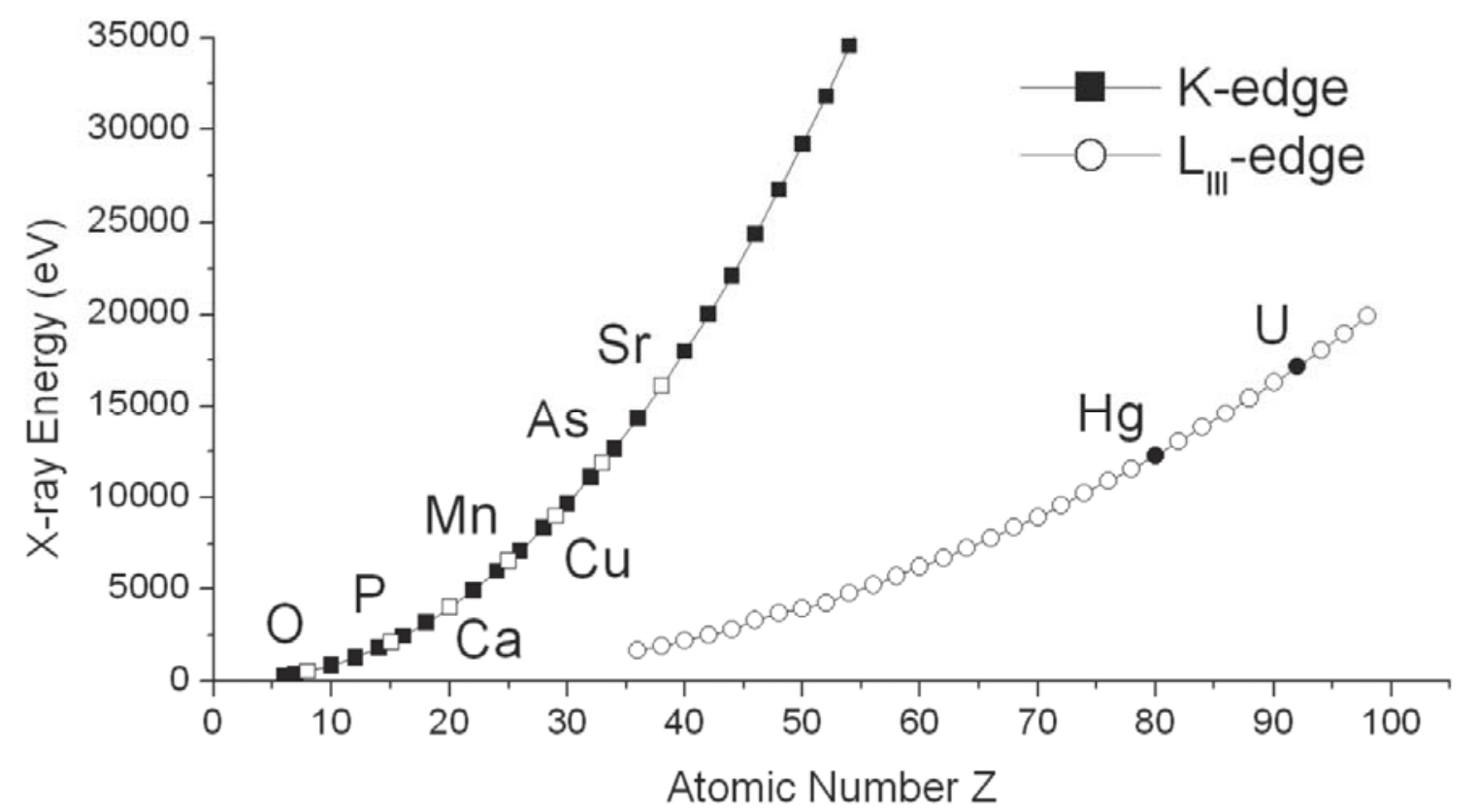

Figure 2.1. X-ray energies for the K- and LIII- edges for various elements as a function of their atomic number. Figure is adapted from Ref 13. 


\begin{tabular}{|l|c|}
\hline Level & Core-Level Excitation \\
\hline K edge & $1 \mathrm{~s} \longrightarrow \mathrm{p}$ \\
\hline $\mathrm{L}_{1}$ edge & $2 \mathrm{~s} \longrightarrow \mathrm{p}$ \\
\hline $\mathrm{L}_{23}$ edge & $2 \mathrm{p}_{1 / 2,3 / 2 \longrightarrow \mathrm{s}, \mathrm{d}}$ \\
\hline $\mathrm{M}_{1}$ edge & $3 \mathrm{~s} \longrightarrow \mathrm{p}$ \\
\hline $\mathrm{M}_{23}$ edge & $3 \mathrm{p}_{1 / 2,3 / 2 \longrightarrow \mathrm{s}, \mathrm{d}}$ \\
\hline
\end{tabular}

Table 2.1 X-ray absorption edges and their correlating core level excitations. 
$\mathrm{E}_{\mathrm{f}}$ and $\mathrm{E}_{\mathrm{i}}$ : final and initial energy states.

This equation is valid for the final state with infinite lifetime. For finite lifetimes, the delta function will be replaced with a Lorentzian. ${ }^{30,31}$

The photoelectron can be represented as a spherical propagating wave, traveling out of the absorbing atom and finally returning to the absorber atom (Fig. 2.2). This propagating wave is scattered by the neighboring atoms of the initial absorber atom. The backscattered wave and the original outgoing photoelectron can interfere; this interference will affect the probability for X-ray absorption by the absorber atom. The outgoing and backscattered wave interference pattern depends on their relative phase. The relative phase is a function of the photoelectron wavelength and the interatomic distances between the absorber and the scattering atoms. Depending on the relative phase of these two wave functions, they can either interfere constructively or destructively. The sum of these two wave functions, which describes the interference pattern, will oscillate with a periodicity that is proportional to the average distance between the absorber and its neighboring atoms.

Neighboring atoms that are at the same distance to the absorber atom are called shells; the number of atoms in a shell equals the absorber atom's coordination number. The scattering of the photoelectron that starts from the original absorber atom can have different configurations, depending on which neighboring atom is the destination of the photoelectron, these configurations are called scattering "path". Two types of scattering are possible here as illustrated in Figure 2.3; single and multiple scattering. Single scattering happens when the path includes the absorber atom and only one scattering atom: the photoelectron is scattered by one other atom and returns to the original atom. This atom 


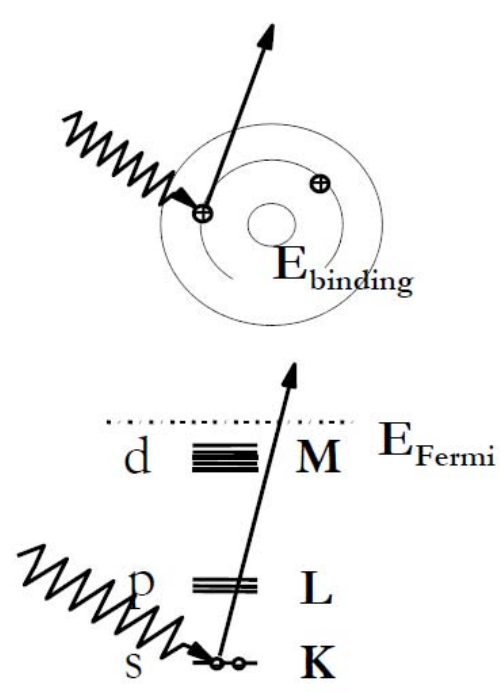

a
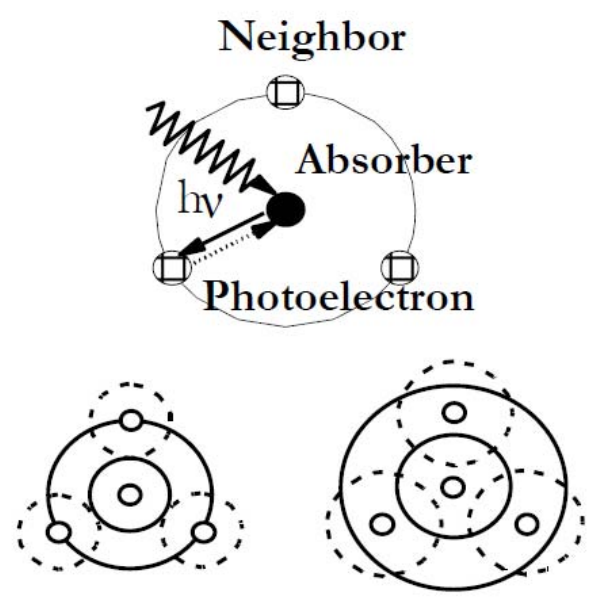

Constructive Destructive

b

Figure 2.2 Schematic of X-ray absorption processes: a) Excitation of a core-level and ejection of a photoelectron, $b$ ) backscattering by neighboring atoms resulting from the ejection of the photoelectron. . Figure adapted from Ref. 36. 


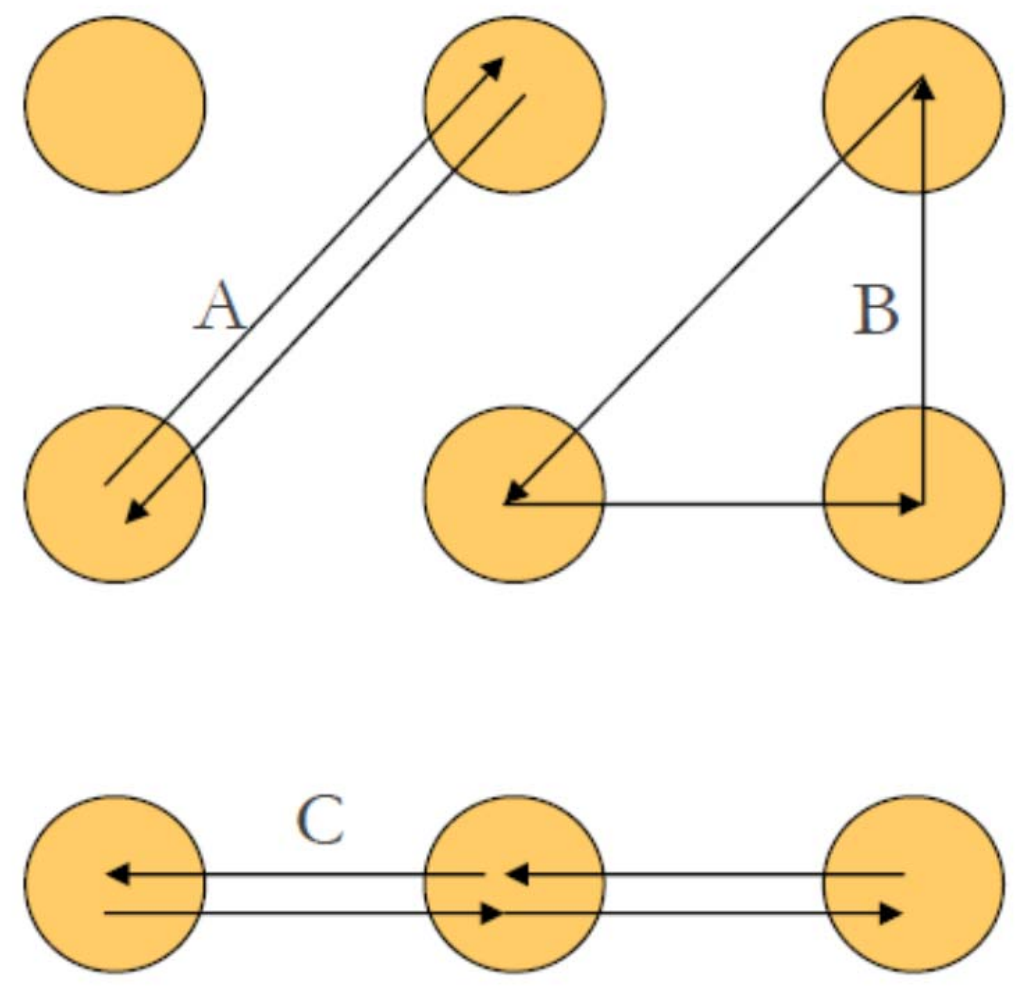

Figure 2.3. Schematic of different backscattering events occurring in an absorption event. 
can be in the first coordination shell or in higher shells. Multiple scattering paths consist of scattering of the outgoing photoelectron to multiple neighboring atoms in any fashion, and finally returning to the original absorber atom. Extremely long multiple scattering paths either cancel each other or experience drastic amplitude reductions through inelastic scattering effects. "Collinear" atoms particularly contribute strongly to backscattering, due to the "focusing" effect caused by them. The degeneracy of a single scattering path is equal to the coordination number of that shell, whereas the degeneracy of a multiple scattering path is actually the number of equivalent paths.

The XAS spectrum is divided in two regions: the XANES (X-ray Absorption Near Edge) and EXAFS (Extended X-ray Absorption Fine Structure) regions. XANES includes the energy range of 0 to $50 \mathrm{eV}$ while the region above $50 \mathrm{eV}$ is called EXAFS. These two regions of the spectrum have the same physical origin but provide different and complementary information. It is generally believed that the XANES region is dominated by multiple scattering processes. In this lower energy region, the photoelectron has a smaller momentum and therefore a longer mean free path. Hence, in this region the photoelectron wave will sample the close neighbors of the absorber many times and multiple scattering will dominate. On the other hand, in the EXAFS region ( energy> 50 $\mathrm{eV}$ ) the photoelectron has a higher energy, inelastic scattering is large, and only single scattering coherent events are of significance. Thus, the data analysis for these two regions and the information provided by these two regions is very different. In the next two sections, details on the data analysis of both of these regions and the types of information obtained from these regions will be discussed in detail. 


\subsubsection{EXAFS}

The EXAFS region is the oscillatory section of the absorption coefficient from above $50 \mathrm{eV}$ up to $1000 \mathrm{eV}$ or higher. This part of the spectrum contains geometrical and structural information, such as coordination numbers, interatomic distances and bond disorder around the absorber atom. As described previously, the oscillatory behavior of the spectrum above the edge energy arises from the interference between the outgoing and backscattered wave functions. The absorption coefficient above the edge energy is mathematically expressed as:

$$
\mu=\mu_{0} \cdot(1+\chi)
$$

where $\mu_{0}$ is described as a smooth atomic background caused by the photon's elastic and inelastic scattering, or in other words the absorption coefficient of the absorber atom itself, and $\chi$ is due to the oscillations caused by the interference of the outgoing and backscattered wave functions. The latter is superimposed on variations of the atomic absorption $\left(\mu_{0}\right)$. $\chi$ , which represents the EXAFS part of the spectrum can then be extracted from the above equation as:

$$
\chi(k)=\frac{\mu(k)-\mu_{0}(k)}{\mu_{0}(k)}
$$

In the above equation the EXAFS is expressed as a function of the wave number of the photoelectron, $\mathrm{k}$. Here, $\mathrm{k}$ is a function of the kinetic energy of the photoelectron. The kinetic energy of the ejected photoelectron $\left(E_{k}\right)$ is equal to the difference between the energy of the incident X-ray beam (hv) and the binding energy of the photoelectron plus the work function of the metal $(\varphi)$ :

$$
\mathrm{E}_{\mathrm{k}}=\mathrm{h} v-\mathrm{E}_{\text {binding }}-\varphi
$$


The wave number is defined as:

$$
k=\frac{2 \pi}{\lambda}
$$

Thus, combining Eqs, 7 and 8 and replacing $v$ with $\mathrm{k}$ will yield:

$$
k=\sqrt{\left(\frac{8 \pi^{2} m}{h^{2}}\right)\left(h v-E_{0}\right)}
$$

where $\mathrm{m}$ is the electron mass and $\mathrm{h}$ is Planck's constant.

The EXAFS Eq. 9, as derived by Sayers, Stern and Lytle, ${ }^{2}$ describes $\chi$ assuming the single scattering, plane wave approximation as: ${ }^{32}$

$$
\chi(k)=\sum_{\mathrm{j}} \frac{\mathrm{N}_{\mathrm{j}} \mathrm{S}_{0}^{2} \mathrm{f}_{\mathrm{j}}(\mathrm{k}) \mathrm{e}^{\frac{-2 \mathrm{R}_{\mathrm{j}}}{\lambda(\mathrm{k})}} \mathrm{e}^{-2 \mathrm{k}^{2} \sigma_{\mathrm{j}}^{2}}}{\mathrm{kR}_{\mathrm{j}}^{2}} \sin \left[2 \mathrm{kR}_{\mathrm{j}}+\delta_{\mathrm{j}}(\mathrm{k})\right]
$$

In Eq. $10, f_{j}(k)$ is the scattering amplitude, and $\delta_{\mathrm{j}}(\mathrm{k})$ is the phase shift of the photoelectron; both of these two parameters are determined by the scattering properties of the neighboring atoms and depend on the atomic number of the scattering atom $(\mathrm{Z})$. The phase of the photoelectron also depends on other factors: 1) while traversing the atomic potential of the absorber atom the photoelectron undergoes a phase change; 2) the potential of the scattering atom; and 3) re-entry of the photoelectron to the absorber atom. $\lambda(\mathrm{k})$ is the mean free path of the photoelectron. All of these parameters can be calculated with a computer program such as FEFF, and the following parameters can be calculated from EXAFS using Eq. 10:

- $\mathbf{R}_{\mathbf{j}}$ : This is the half path length of the photoelectron, which is equal to the distance between the absorber atom and its neighboring atom in a single scattering event. It can also be described as: 


$$
R_{j}=R_{0 j}+\Delta R_{j}
$$

In modeling the EXAFS spectrum, $\mathrm{R}_{0 \mathrm{j}}$ is the half path length utilized by the fitting program and the user can modify this parameter utilizing $\Delta \mathrm{Rj}$.

- $\mathbf{N}_{\mathrm{j}}$ : Number of coordinating atoms in a specific shell.

- $S_{0}^{2}$ : Many body amplitude reduction factor. This value can be different for each path of the photoelectron. After formation of the core hole, the remaining electrons will undergo relaxation; this is taken into account in the amplitude reduction factor. The value for $S_{0}^{2}$ depends on the nature of the element under study. This value is normally defined between 0.07 to 0.1 .

- $\sigma_{j}^{2}$ : This parameter is called the Debye-Waller factor. It is defined as the meansquare displacement of neighboring atoms in a shell. This term is the sum of dynamic (thermal) disorder and static disorder (or structural heterogeneity). ${ }^{29}$ The excitation process and formation of the oscillatory part of the spectrum happens within femto seconds, which is less than the time frame required for thermal vibrations ( $10^{-10}$ to $10^{-12}$ seconds). Thus, during the excitation process the atoms are all fixed at their thermodynamic minima. The EXAFS measures the distribution of interatomic distances between the absorber and scattering atoms within a specific coordination shell.

Eq. 10 easily shows why EXAFS is a "local" structural probe. The term $e^{\frac{-2 R_{j}}{\lambda(k)}}$ shows the exponential fall-off the EXAFS signal with the mean free path of the photoelectron $(\lambda(\mathrm{k}))$. This term causes the EXAFS signal to be dominated by scattering of the photoelectron from atoms within $10 \mathrm{~A}$ of the absorber atom. Further, the EXAFS 
equation also has an inverse dependence on $R_{j}^{2}$. This means that the contribution from shells at increased distances from the absorber atom has a smaller effect on the EXAFS signal. All of these results in XAS being a short range technique, providing information on the immediate environment around the absorber atom, making XAS a very powerful and applicable technique for studying interfacial phenomena on systems like catalysts.

\subsubsection{XANES}

At low energies, the excited photoelectron has a long mean free path, which leads to multiple scattering phenomena dominating the XAS spectrum. The XANES region is specifically representative of the local geometry and oxidation state of the absorber atom. The overall shape of the XANES region is so sensitive to the immediate surroundings of the absorber atom that it can even show the presence of ligands around the absorber atom. Analysis of the XANES region has been tagged as a "fingerprinting technique", as all compounds have their own "signature" lineshapes in this region. For example, the intensity of the Pt $L_{3}$ edge $\left(2 p_{3 / 2} \rightarrow 5 d\right)$ directly follows the d-band vacancy or the density of unoccupied d states just above the Fermi level. This means that the variations observed in the white line intensity can be interpreted as variations in the bonding state of $\mathrm{Pt}$, as the presence of coordination ligands will result in donation or withdrawal of charge to/from the $\mathrm{d}$ band of Pt. However, these changes in the XANES region can be difficult to detect, especially when dealing with small changes in the absorber atom surroundings as observed in surface phenomena in systems like catalysts. These changes can be as small as $3-5 \%$ of the total intensity, and they are also hidden due to the domination of atomic absorption in this region. There are no general equations, like the EXAFS equation, describing the 
XANES region. All these reasons require a new data interpretation technique for XANES data analysis, especially when studying surface phenomena. Ramaker and Konnigsberger ${ }^{3,4,33}$ developed the $\Delta \mu$ technique to analyze the XANES spectra; this technique involves isolation of the changes in the XANES region, and then modeling them with theory.

\subsection{Experimental Methods}

\subsubsection{Synchrotron Radiation}

$\mathrm{X}$-ray absorption spectroscopy experiments are conducted at synchrotron radiation facilities. It has been estimated that there are about 50 synchrotron radiation facilities operating or being constructed around the world. ${ }^{13}$ In the United States, currently there are four DOE-funded Synchrotron radiation facilities: the National Synchrotron Light Source (NSLS) at Brookhaven National Laboratory, the Advanced Photon Source (APS) at Argonne National Laboratory, the Stanford Synchrotron Radiation Laboratory (SSRL) at Stanford Linear Accelerator Center and the Advanced Light Source (ALS) at Berkeley Laboratory. Recently, a third generation light source (NSLS II) is being constructed at Brookhaven National Laboratory.

Figure 2.4 depicts a schematic of a synchrotron radiation facility (NSLS). A linear accelerator is the first step in production of high intensity X-rays: particles are charged and 


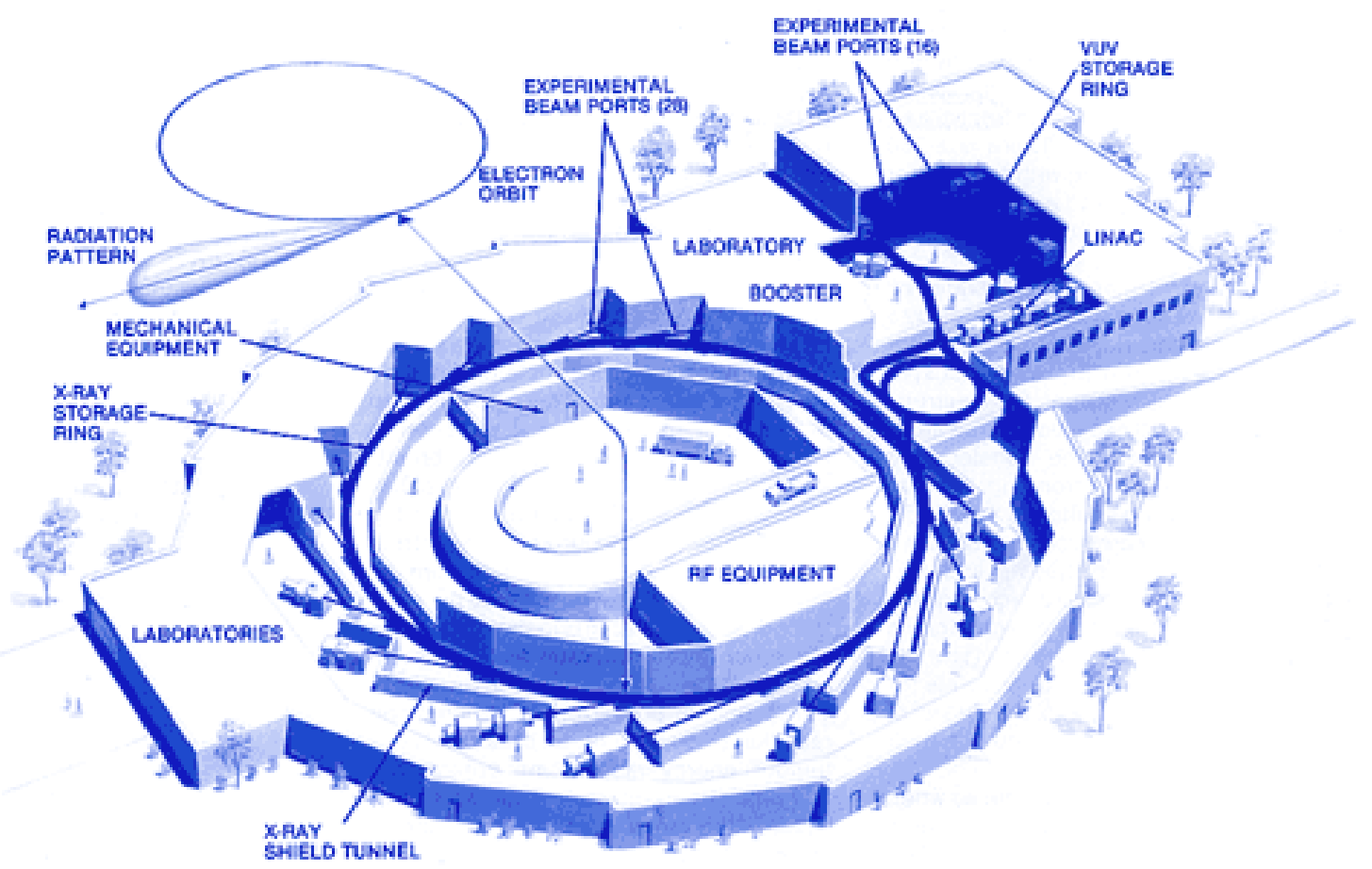

National Synchrotron Light Source

Figure 2.4. Schematic of The National Synchrotron Light Source at Brookhaven National Laboratory, Upton, N.Y. Courtesy of NSLS, Brookhaven National Laboratory (www.bnl.gov) 
accelerated by this linear accelerator. Charged particles are then sent through a booster ring for further acceleration. The booster ring increases the particle's velocity to near the speed of light, and injects them into a storage ring. The particles are constantly accelerated towards the center of the storage ring; this will cause their trajectory to change in a way that would keep them in a closed loop. Conservation of energy requires accelerated charge particles to emit radiation. This emitted radiation is tangential to the particles trajectory, as predicted by Maxwell's laws. To bend the trajectory of particles, magnets are installed in the storage ring. In third generation facilities, wigglers and undulators are utilized in straight sections of the storage ring (consider the storage ring as a polygon with $\mathrm{N}$ sides). Wigglers are devices that increase the X-ray beam's intensity. They consist of a number of bending magnets closely spaced. The particles oscillate when they go through an undulator, the undulator consists of several magnets that are placed periodically. The magnets are arranged in a way that their poles will produce an alternating magnetic field with a specific wavelength that is distributed across the length of the undulator. Particles oscillating in the undulator will emit very intense X-rays with a specific wavelength, and the wavelength of the X-ray beam can be selected by changing the spacing of the magnetic poles. The wigglers and undulators play a big role in preserving the brilliance of the X-ray beam over wide energy ranges.

Beamlines are located at the periphery of the storage ring, collecting X-rays and directing them through a set of slits and monochromator towards the sample. Beamlines contain a sophisticated setup for collecting, selecting and detecting X-rays. Figure 2.5. 


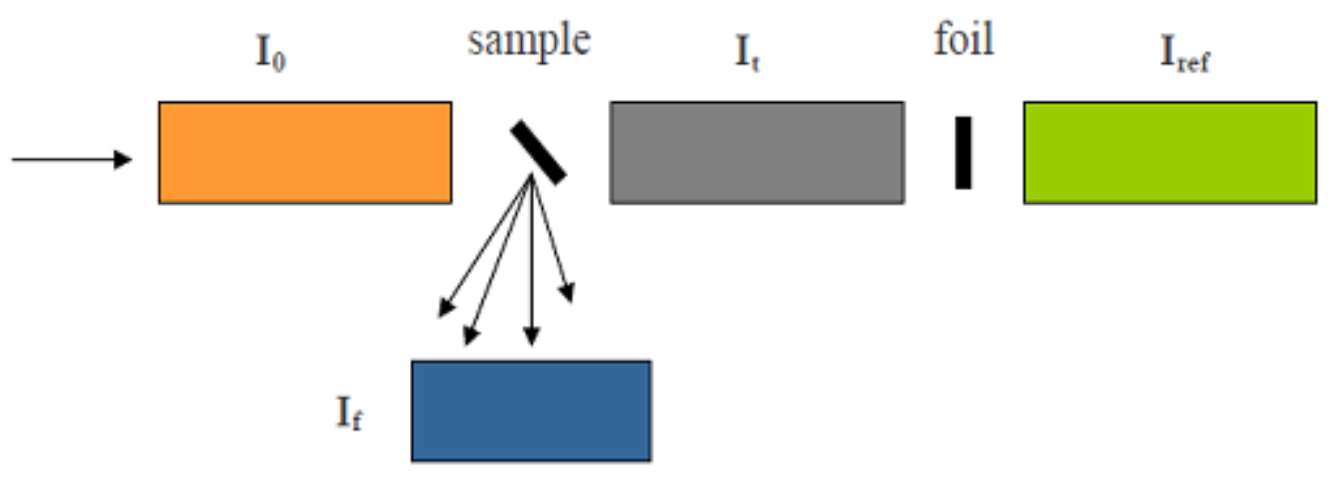

Figure 2.5 Experimental setup at the beamline for transmission and fluorescence measurement modes. Both are possible, with $\mathrm{I}_{\mathrm{f}}$ as fluorescence detector and $\mathrm{I}_{\mathrm{t}}$ as transmission detector. 
depicts a schematic of the beamline setup. A brief overview of the beamline components is presented here.

\section{- Slits}

The main function of slits is to define the physical width of the X-ray beam entering the hutch that contains the sample, and also to block stray radiation. Two types of slits are utilized in beamlines: fixed and adjustable. Fixed slits have a fixed opening and can be moved in when required; whereas adjustable slits have a width that can be adjusted to change the X-ray profile. Beamlines have slits implemented before and after the monochromator. The slit widths need to be adjusted after a wide energy range scan and before starting a new experiment. Slits could also be found before the ionization chambers. Slits are placed both horizontally and vertically. Vertical slits are utilized to increase the energy resolution, while optimizing the beam intensity.

\section{- Monochromator}

Selecting the X-ray energy to shine on the sample is the monochromator's function. Usually a double crystal monochromator, such as depicted in Figure 2.6, is utilized in a beamline setup. Double crystal monochromators consists of two parallel crystals typically made of silicon or germanium. The monochromator is designed in such a way that a particular atomic plane $(h k l)$ of the crystal is exposed to the X-ray beam. X-rays are diffracted from the surface of the crystal following Bragg's Law:

$$
n \lambda=2 d_{h k l} \sin (\theta)
$$




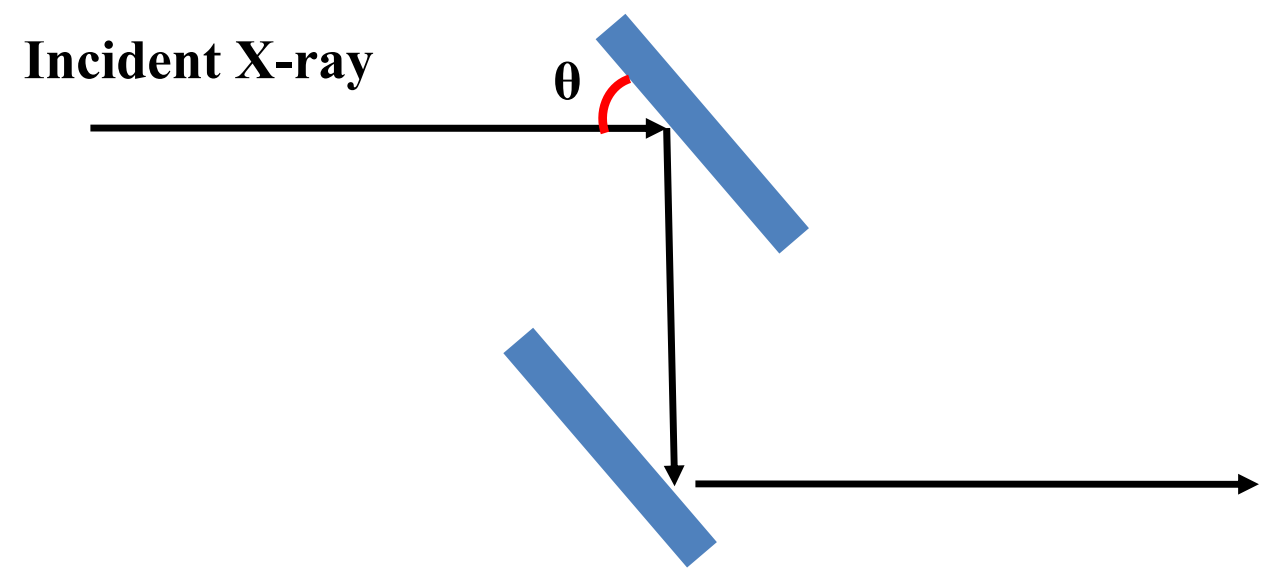

Figure 2.6 Schematic of a double crystal monochromator utilized in tuning the beam energy. 
where $d$ is the lattice spacing of the crystal's atomic plane parallel to the surface, $\theta$ is the angle between the crystal and incident $\mathrm{X}$-ray beam, $\lambda$ is the wavelength at which the $\mathrm{X}$-ray is diffracted and $\mathrm{n}$ is an integer defining the fundamental or harmonic of the X-ray energy. By rotating the monochromator and changing the angle of the incident beam, the energy of the X-ray beam is changed. During an experiment, rotating the monochromator step-bystep allows for scanning of a wide range of energies.

The higher X-ray harmonics must be rejected, as these harmonics can impact the data. One method to reject higher harmonics, especially the second harmonic that is the most intense, is to use a crystal that does not diffract them. Another method to reject higher

harmonics is detuning the second monochromator crystal. Higher crystal lattice planes, which cause higher harmonics to possess a narrower rocking curve, means that a slight change in the second monochromator crystal's angle will decrease the fundamental X-ray intensity slightly, but decrease the harmonics intensity significantly. Another method to reject the higher harmonics is using a "rejection mirror". The mirror is placed at an angle with the beam in a way that would only reflect the fundamental energy to the sample and reject harmonics. The monochromator needs to be calibrated prior to data collection. This is due to two reasons: 1) to account for inaccuracies in the motors that adjust the monochromator angle; and 2) to correct for temperature effects on the lattice spacing of the crystal.

\section{- Detectors}

Inside the hutch (optical closure that contains the sample), the X-ray will travel through three ionization detectors. The sample will sit between the first two detectors $\left(\mathrm{I}_{0}\right.$ 
and $I_{t}$ ) and reference foil will be placed between the second and third ionization detector ( $\left.\mathrm{I}_{\mathrm{ref}}\right)$. The reference foil accounts for the energy drift of the beam over time. Ionization detectors contain a mixture of gasses. Most commonly helium, nitrogen and argon are used in these kinds of detectors. When the X-ray beam hits gaseous atoms, these atoms are ionized. A bias voltage is applied to the walls of the detector, thus the gaseous ions will create a current. The detector will translate changes in the X-ray signal intensity to a electric response measured by the detector. The current produced by the gaseous ions is weak and needs to be amplified to proper levels to be detected. A voltage-frequency convertor digitizes this signal. Amplifier gains are usually in the $108 \mathrm{~V} / \mathrm{A}$ range. To achieve a specific X-ray intensity, y, the ratio of gases in the mixture should be optimized at the beginning of the experiment, noting that heavier gases will attenuate the X-ray beam more.

Fluorescence detectors are placed at a $90^{\circ}$ angle to the incoming X-ray beam, with the sample located at a $45^{\circ}$ angle between them. This arrangement provides for maximized signal to noise ratio, as fluorescence signals are transmitted isotropically and scattering signals are at a minimum at $90^{\circ}$ angle. There are two types of fluorescence detectors commonly used: the Stern-Heald ${ }^{21}$ detectors and solid-state detectors. The fluorescence detector used in this work was a germanium multi-element "solid state" detector. Solidstate detectors are usually either made of germanium or silicon. A thin beryllium window protects the detector. The solid state detector's temperature is controlled by liquid nitrogen. Multi-element detectors consist of multiple sensing elements housed in multiple channels. The fluorescence X-ray signal is detected in each channel during each scan, and will be 
added when analyzing data to obtain the total value of incoming X-ray signal. Multielement detectors have greatly improved the count rate capabilities of detecting systems.

\subsubsection{Beamline Setup}

Beamlines usually consist of two separate enclosures, the first one containing the monochromator while the second one is a hutch that houses the sample positioning stage and detectors. This arrangement keeps the monochromator protected and at a constant and controlled temperature. Users can access the second hutch to setup the sample stage and run in-situ and ex-situ measurements. When significant X-ray absorption by the air is observed, tubes filled with helium can be installed to minimize these effects. Cryostats are available in some beamlines, such as the X3-B beamline at NSLS, for experiments that require a fixed temperature over the sample. Optical equipment such as the slits and mirrors are installed both in the monochromator enclosing and the second hutch. All optical systems need to be optimized prior to conduction of experiments to maximize the X-ray intensity and resolution.

X-ray absorption experiments can be conducted in two different modes: transmission and fluorescence. The selection of the measurement mode is based on the nature and concentration of sample. More concentrated samples are subject to transmission measurements. The criterion for choosing this mode is that an absorption step height of $\sim 1$ should be reached. For samples showing step heights less than 0.2, with high signal to noise ratios, fluorescence mode measurements are best suited. Before conducting the XAS experiment, users should calculate the required amount of sample that would yield the 
necessary theoretical absorption step height values. These calculations can be carried out using widely available reference tables that contain energy dependent mass absorption coefficients and effective cross-sections for many elements in a wide range of energies. ${ }^{5-11}$ An alternative approach is to use the HEPHASESTUS program in the IFEFFIT suite, which calculates absorption step heights for samples easily. ${ }^{22}$

When conducting fluorescence measurements, it should be noted that the relationship between the absorption coefficient and incident and transmitted beam intensities is more complex than that in transmission mode. The equation defining this relationship is as follows:

$$
I_{f}=\frac{I_{0} \varepsilon \Delta \Omega \mu_{\chi}(E)\left\{1-e^{-\left[\frac{\mu t(E)}{\sin \theta}+\frac{\mu t\left(E_{f}\right)}{\sin \varphi}\right] t}\right\}}{\mu_{t}(E) / \sin \theta^{+}{ }^{\mu_{t}\left(E_{f}\right)} / \sin \varphi}
$$

Here $\mathrm{I}_{\mathrm{f}}$ and $\mathrm{I}_{0}$ are the emitted and incident X-ray intensities, $\varepsilon$ is the fluorescence efficiency, $\mathrm{t}$ is the sample thickness, $\Delta \Omega$ is the angle between the emitted fluorescence and the detector, $\mu_{x}(E)$ is the absorption coefficient of the element of interest, $\mu_{t}(E)$ is the total absorption, $\theta$ and $\varphi$ are the incident and reflected X-ray beam angles, and $E_{f}$ is the fluorescent beam energy. When dealing with thin samples (as often is the case when conducting fluorescent measurements) with $\mu_{\mathrm{t}}<<1$, the exponential term will be reduced to " $\mathrm{t}$ ":

$$
I_{f}=I_{0} \frac{\epsilon \Delta \Omega}{4 \pi} \mu_{\chi}(E) t
$$


In the case of dealing with a thick and dilute sample, $\mu_{x}<<\mu_{t}$ and the energy dependence of $\mu_{\mathrm{t}}$ is negligible, thus Eq. 14 reduces to:

$$
\mu_{t} \approx I_{f} / I_{0}
$$

This equation is most commonly used in fluorescent data measurements.

\subsubsection{Design of X-ray Absorption Experiment}

This dissertation is focused on studying electrochemical processes in alternative energy devices (fuel cells and batteries) utilizing XAS. Throughout this dissertation two types of experiments were conducted: in-situ and ex-situ. Chapters 3 and 4 are concentrated on in-situ studies of adsorption of poisons on electrodes in fuel cells. Chapters 5 and 6 are focused on ex-situ studies of super-iron salts as multi-electron cathodes for high capacity batteries. Both of these experimental setups will be discussed here briefly:

\section{- In-situ Experiment}

It was mentioned previously that because X-rays are the probe in XAS, this technique is element specific and can be conducted in complex environments such as an operating electrochemical cell. Over the years the in-situ cell design has improved. The latest version of the in-situ cell employed in our studies allows for saturating the electrolyte with any gas $\left(\mathrm{O}_{2}, \mathrm{~N}_{2}\right.$ or Ar). Specific concentrations of poisons can be introduced to this cell under controlled electrochemical conditions. This cell is a flow-through ambidextrous cell, with the electrolyte reservoir on its vertical face. This allows for both transmission and 
fluorescent measurements. The fact that the cell is ambidextrous allows for use of this cell in any beamline and any sample stage orientation with all types of detectors. The cell does not necessarily need to be operated in "flow-through" mode and this feature can be switched off. The working electrode and counter electrode are placed across from each other and separated uniformly by about $4 \mathrm{~mm}$. The uniform separation of the electrodes is necessary to avoid non-uniform polarization of the electrodes. This is specifically undesired because it could cause the appearance of "dead-zones" on the electrode, which will result in regions of the electrode having altered surface chemistry. The counter electrode is often a carbon-based electrode. The reference electrode is placed in a separate reservoir and ionic contact between this electrode and the other two electrodes is maintained through the use of a salt bridge. There is a risk that the reference electrode might release ions into the solution and poison the electrode. For this reason it is often preferred to use a standard hydrogen electrode (SHE). A peristaltic pump is used to circulate the electrolyte into the reservoirs around the electrodes. High-quality gaskets are used then to seal the cell and prevent leakage. The latest version of the in-situ cell is equipped with a secondary container that allows for leaked electrolyte to flow in it and prevent leakage of potentially harmful electrolyte into the beamline setup. Three pegs built on the secondary containment facilitates the re-alignment of the cell each time it is moved in and out of position. The presence of gas and air bubbles in the X-ray window region is a serious problem and can severely impact data quality. To avoid this, the peristaltic pump and in-situ cell should always be positioned at the same height. The X-ray window is 


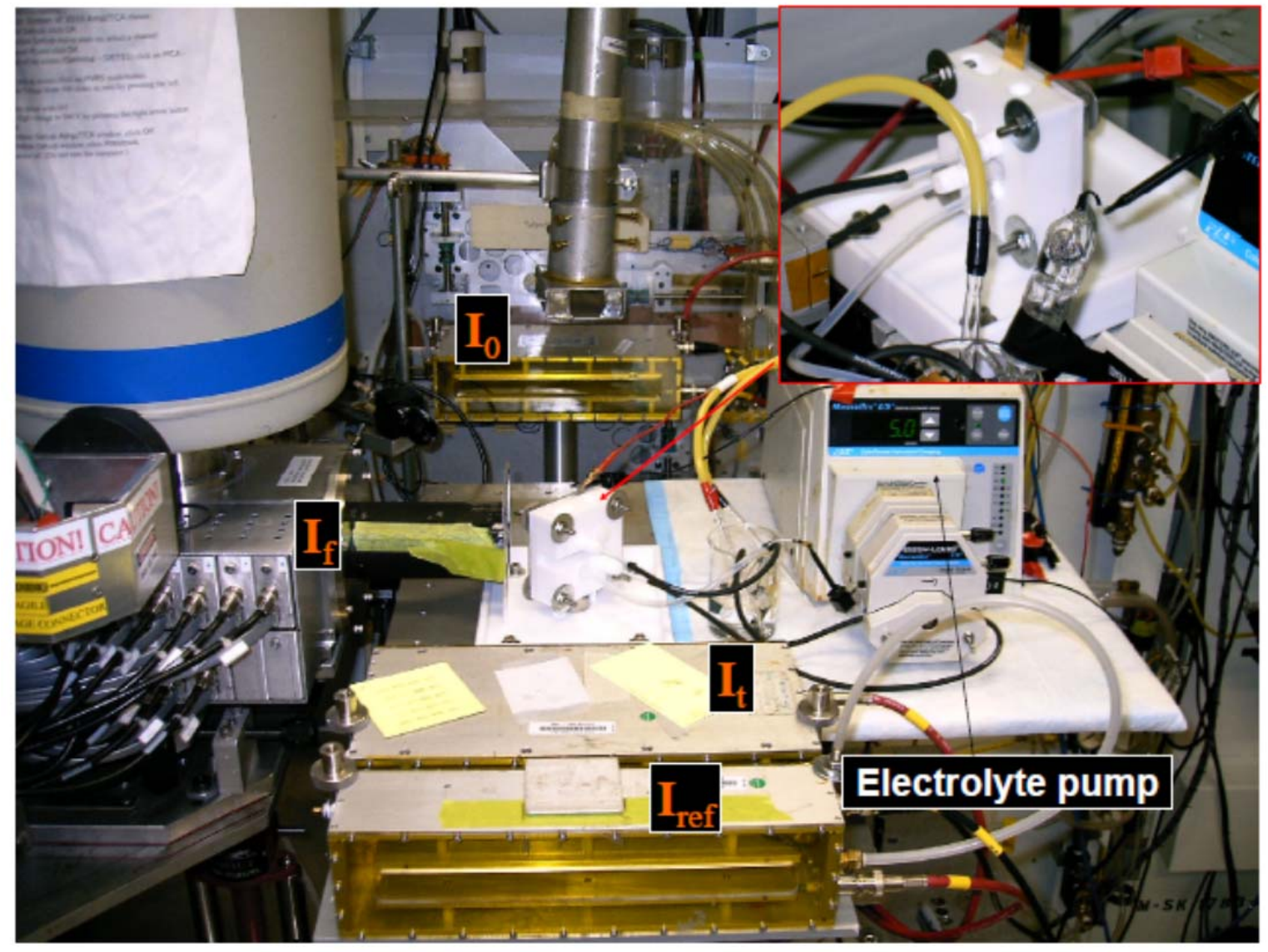

Figure 2.7. X3-B beamline setup at NSLS, when running an in-situ XAS experiment using a flow-through cell. 
sealed with a PTFE tape $(3 \mathrm{M})$ along with a silicone adhesive. This tape is transparent and provides a tight seal for the X-ray window and is highly stable at higher energy range.

However, it shows accelerated degrading at lower edge energies. Figure 2.7 shows an in-situ cell inside the X3-B beamline at NSLS.

\section{-Ex-Situ Experiment}

In this dissertation, ex-situ experiments were conducted to study novel cathode materials for high capacity batteries. These materials existed in both micron and nano size domains. Conducting ex-situ measurements are easier and less complex and sensitive than in-situ experiments. However, it is of crucial importance to ensure uniformity across the sample. Prior to conducting these types of measurement, it is necessary to calculate the appropriate sample size that would yield a satisfactory absorption step height. If a powdered sample is being studied, it should first be ground in a morter and pestle and then sieved through a mesh. This will ensure uniform particle size distribution throughout the sample. The sieved powder was then painted and sandwiched between two pieces of Kapton tape. In case of studying composites such as a cathode mixture, the electrode was scraped and painted between two pieces of Kapton tape. The ex-situ sample is then mounted onto a sample holder with appropriate height. Extra care should be exercised to mount the sample aligned with the first and second ionization chambers at maximum X-ray intensity. A "burn" test can show if the sample is mounted at the correct position, with Xrays going through it. 


\subsection{Data Analysis}

\subsubsection{EXAFS Data Analysis}

Before proceeding to review details of the EXAFS data analysis, a review of the assumptions in deriving the EXAFS equation can be helpful:

1. Within a given shell, atomic potentials are all "almost" the same;

2. To model the energy distribution around the absorber atom "muffin tin" potentials are used at certain and fixed distances;

3. It is assumed that only one photoelectron is ejected as a result of the photon absorption process;

4. The photoelectron is regarded as a "plane" wave;

5. Only single-scattering events are of importance and multiple-scattering is omitted.

Processing and analyzing the EXAFS spectra will yield valuable structural information on the samples under study. This section describes the data analysis procedure to obtain the desired parameters from the EXAFS equation described in the previous section. The software used to analyze the data is the IFEFFIT $^{(}$suite, ${ }^{12}$ an interactive program with algorithms for fitting the experimental data to theoretical calculations. This software package includes a number of different programs; in this dissertation two of these programs are extensively used: ATHENA and ARTEMIS: ${ }^{22}$

\section{- ATHENA}

This program is a GUI to IEFFEIT designed by Ravel and Newville for initial data processing. ATHENA is used for background removal, normalization, alignment, 
calibration and extraction of the EXAFS $\chi(\mathrm{k})$ function. This program is also used in $\Delta \mu$ XANES data analysis.

\section{- ARTEMIS}

This program is also another GUI to IFFEFIT and FEFF designed by Ravel and Newville that is used to fit a theoretical model to experimental EXAFS spectra. The input to this program is the EXAFS $\chi(\mathrm{k})$ function extracted from ATHENA. ARTEMIS will perform a nonlinear regression procedure on the experimental spectrum and a theoretical model which needs to be defined by the user. The user also needs to define and set the parameters that will be used for the fit. The output will be the best fit results along with statistical parameters indicating the quality of the fit. Both ATHENA and ARTEMIS can easily present the data in energy, $\mathrm{k}$ or R space. ARTEMIS performs Fourier transformation on the spectra in a variety of "windows". Commonly used window functions are the Hanning and Keiser-Bessel. These windows are defined by minimum and maximum values for $\mathrm{k}\left(\mathrm{k}_{\min }\right.$ and $\left.\mathrm{k}_{\max }\right)$ and $\mathrm{dk}$ which is the width of the $\mathrm{k}$ region.

The EXAFS data analysis procedure is described step-by-step here and illustrated in Fig. 2.8:

\section{- Background removal and Normalization}

Before carrying out the normalization process, it is desirable to ensure that all spectra have the same $\mathrm{E}_{0}$. The edge energy is the energy required for excitation of a photoelectron to unoccupied states or the continuum with creation of a core hole. The absorption occurs not on a single precise value, but rather over a range. Therefore, it is of benefit to align all experimental edge energies to the theoretical energy reference $\mathrm{E}_{0}$. This ensures that all 
data sets have the same energy shift. $E_{0}$ is the inflection point on the derivative spectrum: $\partial \mu / \partial \mathrm{E}$

Normalization is carried out to account for differences between the spectra that arise due to variations in sample, such as concentration and thickness of the sample, and also to account for variations in detector response. After normalization, the spectra represent absorption per atom; therefore, the differences between experimental spectra represent the differences in the local structure surrounding the absorber atom. The normalization process involves dividing the experimental spectrum by the absorption step height at the edge energy, $\mathrm{E}_{0}$, followed by subtracting the pre-edge background and extending it to the entire spectrum. The pre-edge background is obtained through regression of a linear function in the -200 to $-30 \mathrm{eV}$ region. Another background, called the post-edge background is found by regression in the post edge region $(50-1000 \mathrm{eV})$ as a linear or quadratic function, now extrapolated down to the edge energy. The difference between extrapolation points of these two background lines is called the "edge step". After normalization, the pre-edge region of the normalized spectrum should all be at zero absorption and the edge step should be 1. Subtracting the pre-edge background eliminates effects of higher beam harmonics, elastic scattering, and the unwanted absorption occurring from lower step edges.

\section{- Extraction of $\chi(k)$ function}

Recall that the absorption coefficient of the spectrum equals:

$$
\mu(E)=\mu_{0}(1+\chi)
$$

To extract $\chi$, the normalized oscillatory part of the spectrum that contains all the structural information, the atomic absorption coefficient $\left(\mu_{0}\right)$, must be subtracted from the 
absorption coefficient. This step is achieved with a post-edge background removal. As described previously, the post-edge background removal involves the elimination of the very low-frequency atomic absorption component through the regression of a linear or quadratic line to the post edge region (50 to $1000 \mathrm{eV}$ ). This step is included as a routine in recent versions of standard data analysis programs, yet close attention must be paid to this step as ineffective removal of this component can lead to unforeseen peaks at small $\mathrm{R}$ values in the Fourier-transformed data. Alternatively, extraneous background removal can lead to loss of important structural information.

\section{- Fourier transform of $\chi(\mathbf{k})$}

The extracted $\chi$ function is subjected to Fourier transform. As illustrated in Eq. (10), the EXAFS signal is comprised of the sum of sine waves. Each sine function has an amplitude and phase that are dependent on the interatomic distances (R) and coordination numbers, as well as the nature of the neighboring atoms. When conducting the Fourier transform, the sine waves are separated into their frequency component. Here, the Fourier transform will separate the EXAFS data in terms of $\mathrm{R}$, or the interatomic distance, component following the Nyquist theorem. ${ }^{13}$ The Fourier transform can be described by the following equation:

$$
\chi(R)=\frac{1}{\sqrt{2 \pi}} \int_{k_{\min }}^{k_{\max }} k^{n} \chi(k) e^{i 2 k R} d k
$$

As can be observed above, with the Fourier transform the data is effectively transferred from k-space to R-space. The Fourier transform's magnitude contains peaks in R, these peaks are directly related to the interatomic distances. Within a coordination shell, all atoms that are located at the same radial distance from the absorber atom contribute to the 
Fourier transform magnitude. However, it should be noted that the FT EXAFS magnitude is not exactly a "radial distribution function", rather it just resembles such a function. This resemblance is in the aspect that usually there is a peak that correlates to the first coordination shell and the $\mathrm{R}$ value of this peak is approximately equal to the bond length of that shell. It is only approximately equal to the $\mathrm{R}$ values, because the position of the Fourier transform peaks are slightly shifted to lower $\mathrm{R}$ values when compared to true bondvalues. This arises from the phase term in the EXAFS equation. The $\mathrm{k}^{\mathrm{n}}$ term in the Fourier transform function is a weighting factor for the EXAFS signal to dramatically expanding the very small magnitude of $\chi(\mathrm{k})$ at large $\mathrm{k}$.

\section{- EXAFS fitting and constructing theoretical models}

Extracting structural data from the EXAFS spectrum requires building a theoretical model of the structure surrounding the absorber atom. The experimental EXAFS data will then be fit with the theoretical model to obtain structural information (e.g.: coordination number, bond length, etc.). The IFEFFIT suite of programs developed by the University of Washington is generally utilized for this purpose (Version 1.2.11 IFEFFIT Copyright 2008, Mathew Newville, University of Chicago, http://cars9.uchicago.edu/ifeffit).

Constructing the theoretical model requires defining the initial arrangement of the atoms surrounding the absorber atom by the user for FEFF. This initial arrangement can be obtained either through crystallographic data of the compound under study (such as a .cif file) or through results obtained from density functional theory calculations. Within IFEFFIT itself, there is a built-in coordinates database of a number of commonly encountered structures of compounds, stored under "atomsdb" folder. Further, the program 
Artemis has an interface called "ATOMS" ${ }^{\prime 23}$ that is capable of converting crystallographic information to $\mathrm{x}, \mathrm{y}, \mathrm{z}$ coordinates. The ATOMS code requires the space group, unit cell dimensions, core atom type, absorption edge and fractional positions of the compound as input. The output will be the $\mathrm{x}, \mathrm{y}, \mathrm{z}$ coordinates of the atoms with respect to the absorber atom. The FEFF code needs two other inputs to build the theoretical spectrum: to define the core "HOLE" created (e.g. K, $\mathrm{L}_{\mathrm{III}}$ edges) and the independent potentials "list" of the atoms around the absorber atom. This list will specify the atomic numbers and types of all the atoms in the cluster under study, and assign an "ipot" number to them. The "ipot" number for the absorber atom should always be zero. The "ipot" list is followed by the coordinates list created by "ATOMS" or provided by the user. FEFF will then create muffin tin potentials ${ }^{34}$ about the position of atoms. The output from the FEFF code will generate a list of possible scattering paths and their associated amplitudes. The user can then select which paths to be included in constructing the structural model by Artemis. Once appropriate paths are selected by the user, mathematical expressions of the EXAFS equation (Eq. 10) should be defined and constrained. Next, a linear least-square procedure will be utilized to adjust the user defined variables and mathematical parameters to obtain a fit between the experimental spectrum and the theoretical model. The variables calculated by the EXAFS fit usually include: $\mathrm{N}, S_{0}^{2}, \sigma^{2}, \Delta \mathrm{E}_{0}, \mathrm{R}$ for each path. The parameters, such as scattering factors $\mathrm{f}(\mathrm{k})$ and phase term $\delta(\mathrm{k})$ are either theoretically generated by FEFF or can be obtained through fitting some known standard spectra of the same element under study; this requires the use of a standard compound with known oxidation state and structure. 
Along with the best-fit values for user defined variables, Artemis will also report uncertainties in these variables as well as statistical parameters on the goodness of the fit. These statistical parameters are the number of variables $\mathrm{N}_{\mathrm{var}}$, number of independent points $\mathrm{N}_{\text {idp }}, \chi^{2}$ (chi-square), $\chi_{v}^{2}$ (reduced chi-square) and an $\mathrm{R}$ factor. ${ }^{14,15}$ The $\mathrm{R}$ factor is calculated by dividing the sum of the differences between the experimental data and fit at each point by the sum of squares of all data. For a fit to be considered reasonable, generally R factors need to be less than 0.05 .

The uncertainties in reported values for $\mathrm{N}, \mathrm{R}$ and $\sigma^{2}$ are largely determined by the quality of the fit as well as the quality of the data itself. However, it is common to yield bond distances to an accuracy of $0.02 \mathrm{~A}$ and a $10-15 \%$ error on coordination numbers when compared to known values. In fact when compared to bond distances obtained from XRD, the bond distances reported through XAS may be more accurate. ${ }^{24-28}$ This could be the result of XAS being a very sensitive short range technique. As mentioned previously $\sigma^{2}$ ( the Debey-Waller factor) is the sum of two forms of disorder: static and dynamic disorder. This value is normally higher for alloys, ions and high temperature experiments compared to that of homogeneous materials at normal conditions.

In-situ XAS studies on electrochemical interfaces often involve a more detailed fitting procedure than normal EXAFS fitting.

Our research usually involves following the changes in coordination number $(\mathrm{N})$ as a function of applied electrode potential (V). There is a high degree of correlation between obtained values for the coordination number and Debey-Waller factor $\left(\sigma^{2}\right)$, this means that comparing $\mathrm{N}$ values at different potentials should be done with extra care to ensure that 
variations in $\mathrm{N}$ are solely due to variations in $\mathrm{V}$ and not an effect created by widely

different $\sigma^{2}$ s. Thus, to ensure meaningful $\mathrm{N}$ vs. $\mathrm{V}$ graphs, the following procedure is often followed: All data taken at different potentials are first fit by allowing all four parameters to vary independently. The average value of $\left\langle\sigma^{2}\right\rangle$ obtained through this first set of fitting is then determined. Then a second set of fitting is performed, with the Debey-Waller factor set at the average value determined through the first fitting set, and the other three parameters allowed to vary independently. Although this procedure might compromise the quality of fits, since there are less independent variables for each fit, this procedure allows for a more meaningful and realistic comparison of $\mathrm{N}$ values at different potentials.

\subsubsection{XANES Data Analysis}

The $\Delta \mu$-XANES method is best for applications to systems where there is high control over the immediate environment around the absorber atom. This method has been utilized successfully by several researchers in electrocatalysis and gas-phase catalysis to study the surface chemistry of these systems. This technique is a powerful data analysis technique because it can isolate scattering caused by adsorbate species on the surface of an electrode and follow the adsorption process as the system is under electrochemical operation. In this dissertation, this data analysis method has been used extensively to study two types of electrochemical cells: fuel cells and batteries. Details of this data analysis technique are presented here.

\section{$\Delta \mu$-XANES Method}


The $\Delta \mu$-XANES data analysis should be started with pre-edge background removal and normalization, this step is similar to the first step in EXAFS analysis (see Fig. 2.8). Both of these two steps are performed by Athena and through the same procedure previously described for EXAFS analysis. The main difference is that normalization in the XANES region is done over a smaller energy range, normally 20 to $100 \mathrm{eV}$ relative to the edge energy. After normalization and background removal, all reference foil spectra should be energy calibrated and aligned with a standard reference spectrum (for instance a foil spectrum for which the edge energy, $\mathrm{E}_{0}$, has been calibrated to the known edge energy). This alignment step overcomes energy shifts observed in the spectra due to mechanical instabilities in the monochromator and the energy drift of the beam over time. All theshifts applied to the foil spectra in the alignment step are automatically translated to the sample spectra by ATHENA. The alignment step is a vital step for the success of the $\Delta \mu$-XANES technique, as all atomic contributions need to be cancelled out and all changes observed in the difference spectra constructed in the next step should be representative of real changes in the sample and not variations in beam energy. Next, a "reference" spectrum is selected from all the sample spectra collected. Usually, the spectrum representing the electrode at its cleanest state is selected as "reference" spectrum, for instance the spectrum taken at potential of $0.54 \mathrm{~V}$ or "the double layer" region is considered to be free of adsorbates through electrochemical measurements. The reference spectrum is then subtracted from all spectra, constructing a set of difference spectra:

$$
\Delta \mu=\mu(\mathrm{Ad} / \mathrm{M})_{\mathrm{V}}-\mu(\mathrm{M})_{\mathrm{Vref}}
$$




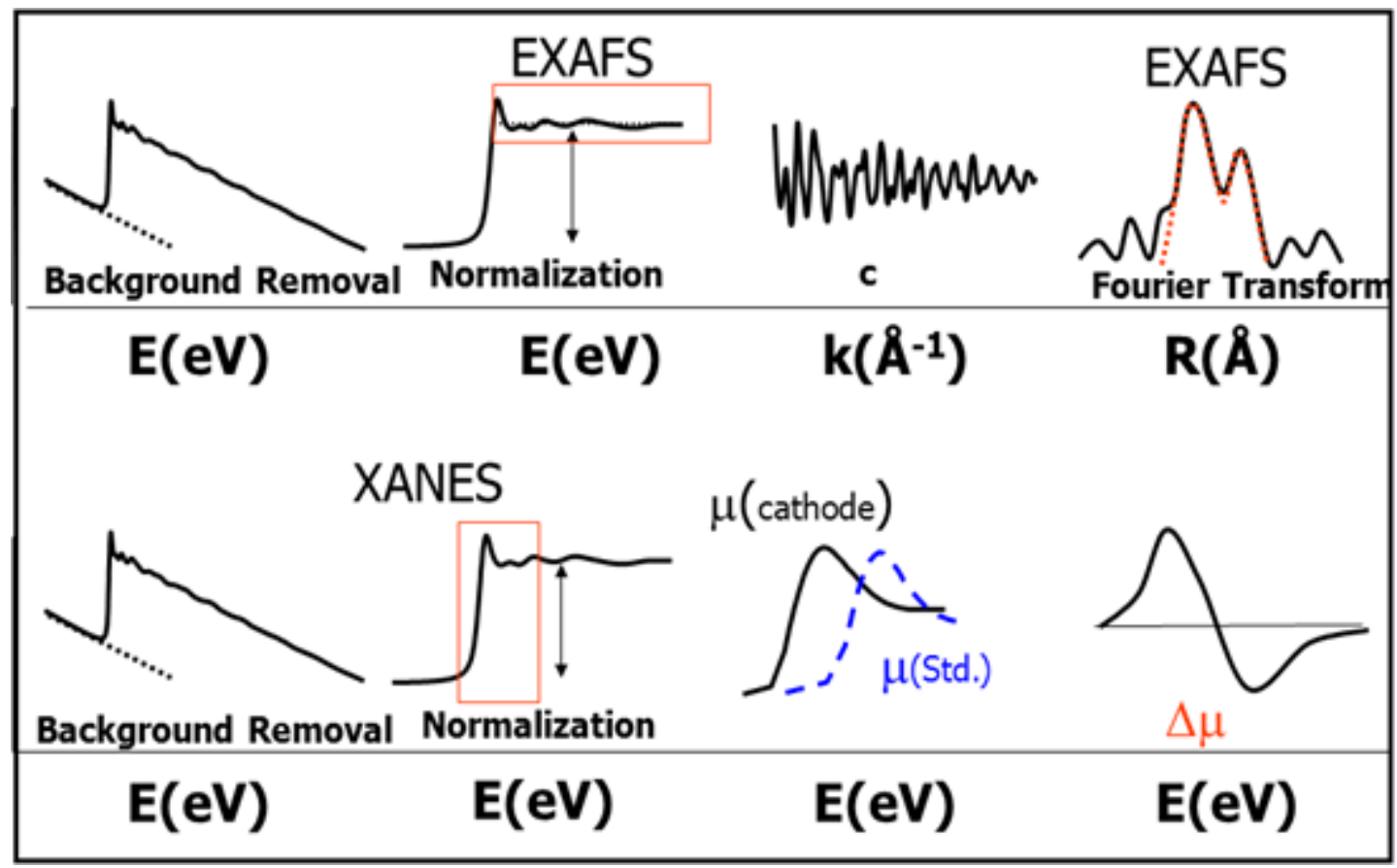

Figure 2.8. Schematic of data analysis procedures for both the XANES and EXAFS techniques. Figure adapted from Ref. 35. 
where $\mu(\mathrm{Ad} / \mathrm{M})_{\mathrm{V}}$ refers to the absorption coefficient of the sample $(\mathrm{M})$ in the presence of adsorbates (Ad), at a specific potential (V), and $\mu(\mathrm{M})_{V \text { ref }}$ refers to the absorption coefficient of the "reference" spectrum at the cleanest electrode potential $\left(\mathrm{V}_{\text {ref }}\right)$. Through this equation, the absorption contribution from the un-reactive bulk should be effectively subtracted out and only the contribution from adsorbates on the electrode surface should remain. The $\Delta \mu$ spectra generated will then reflect the oxidation state, coverage and site symmetry of the adsorbates. These $\Delta \mu$ spectra can also provide a measure of the accessible electrode sites. $^{16-18}$ This is because the $\mu$ contribution from unavailable electrode sites such those sites in contact with support or neighboring particles, sites covered by surface islands, or out of the 3-phase boundary region are also eliminated in the process of making these difference spectra.

The $\Delta \mu$ technique is an extremely sensitive technique. $\Delta \mu$ 's sensitivity goes as low as a fraction of a percent. It can identify various species and permits direct observation of even weakly adsorbed species. Although highly interacting ligands such as metal-oxygen covalent bonds can be easily determined and revealed by this technique, these types of interpretations are restricted by the amount of change in the white line. For instance it is not possible to distinguish between $\mathrm{O}$ and $\mathrm{CO}$ adsorption just from the whiteline. Therefore, only qualitative information can be inferred from analysis of the changes of whiteline in the -50 to $50 \mathrm{eV}$ region. However, $\mathrm{O}$ and $\mathrm{CO}$ can be differentiated using the $\Delta \mu$ technique because $\mathrm{CO}$ and $\mathrm{O}$ have different spectral signatures.

Sometimes when performing $\Delta \mu$ analysis, the background is not completely eliminated. For example the $\Delta \mu$ shows a non-zero slope or it seems like $\Delta \mu$ is on a slowly 
varying background. Performing a Savitzky-Golay ${ }^{19}$ smoothing utilizing $30-50 \%$ of the points can easily remove this "residual background". This procedure is very similar to post-edge background removal, but now it can be extrapolated to even below the edge as the edge step is eliminated when making the difference spectrum. This smoothing procedure can also be applied to reduce the noise level in the data; only a smaller number of points (2-3\%) should be used in the Savitzky-Golay smoothing for this purpose.

Theoretically calculated $\Delta \mu$ lineshapes are required in order to successfully interpret the experimental $\Delta \mu$ spectra. Comparison and correlation of theoretical $\Delta \mu$ signatures will reveal the nature of the adsorbate species, adsorption site and symmetry and in some cases (such as described in chapter 3 of this dissertation) the nature of metal-adsorbate bond. These theoretical calculations are conducted using the FEFF8.0 code. The FEFF 8.0 code uses muffin-tin potentials for real space, full multiple scattering ab-initio self-consistent field calculations. Utilizing a Hedin-Lundqvist exchange correlation approximation, FEFF 8.0 can approximate the Fermi level (or the continuum orbitals) and the full multiple scattering also enables this code to effectively approximate the strong antibonding resonances. FEFE8.0 can include a core-hole on the absorber atom when simulating the excited state of absorber atom. However, the inclusion of a core hole is not necessarily the best approach to simulate the final state, for instance in case of dealing with an absorber atom with a highly occupied valence band the valence electrons can possibly shield the core hole and mask its effects. In these instances, the exclusion of a core hole can yield better agreements with experimental results. 
To simulate $\Delta \mu$ spectra, a "clean" 6 atom cluster, such as the Janin cluster ${ }^{20}$ is used. A XANES spectrum is calculated for this cluster using FEFF 8.0. Next, the adsorbate of interest (e.g.: $\mathrm{H}, \mathrm{OH}, \mathrm{CO}$ ) is placed on the cluster at a specific adsorption site using crystallographic information such as bond lengths to input the coordinates of this adsorbate to FEFE8. Figure 2.9 shows atop, bridge bonded and n-fold adsorption configurations on a 6 atom Pt cluster. Now, a second set of calculations are conducted to simulate the XANES spectra with inclusion of an adsorbate on the cluster. After constructing these two sets of theoretical XANES spectra (one clean and one with the adsorbate) the theoretical $\Delta \mu$ spectrum can be made through:

$$
\Delta \mu_{\text {theoretical }}=\mu\left(\operatorname{ads} / \mathrm{Pt}_{6}\right)-\mu\left(\mathrm{Pt}_{6}\right)
$$

Comparison of this theoretically simulated $\Delta \mu$ spectrum with experimental spectra, will lead to identification of features in the experimental spectrum. Information obtained with this technique, should be correlated with electrochemical or other spectroscopic measurements to ensure their validity.

An important characteristic observed in $\Delta \mu$-XANES spectra of many adsorbates is the strong dependence of spectral features on the adsorption site. This is well explained by expanding $\Delta \mu$ into its component: Now, deriving the above equation will yield:

$$
\begin{gathered}
\mu(E)=\mu_{0}(E)[1+\chi(E)] \\
\Delta \mu=\Delta \mu_{0}+\Delta\left(\mu_{0} \chi_{P t-P t}\right)+\mu_{0, A d s / P t} \chi_{P t-A d s}
\end{gathered}
$$




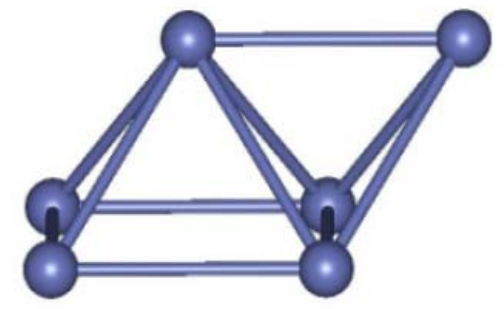

Plain Pt cluster

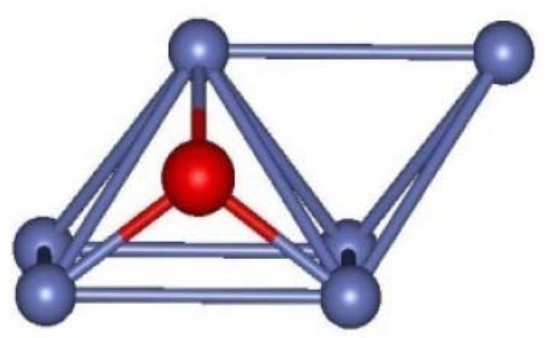

3-fold site

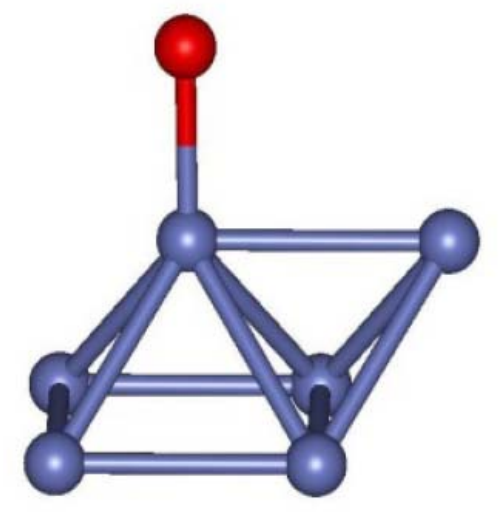

1-fold, atop site

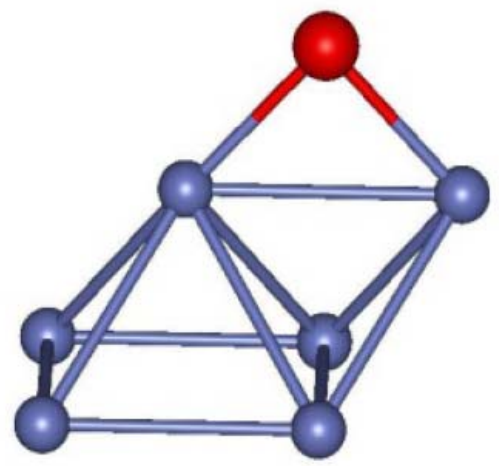

2-fold, bridge-bonded site

Figure 2.9. Adsorption sites commonly observed for small adsorbates. 
Here $\Delta \mu_{0}$ is the change in atomic XAFS caused by the adsorbate coverage, $\Delta\left(\mu_{0} \chi_{P_{t}-P_{t}}\right)$ is the change in Pt-Pt scattering caused by the adsorption, $\mu_{0, A d s / P_{t}}$ is the additional scattering between platinum and the adsorbate atom. It has been shown through FEFF 8.0 theoretical calculations that the Pt-Pt scattering term (the second term) contributes the most to the total $\Delta \mu$ signal. This term provides the sensitivity to adsorbate binding site.

Adsorption of an atom will change the electronic charge distribution and density between the absorber atom and it's neighbors. The change in electronic distribution and density will also change the photoelectron absorption cross section, which means that the backscattering properties change subsequently. Different adsorption sites have different quantities of electron sharing between the adsorbate and the absorber atom, for instance 3fold and bridge bonded sites have higher electron sharing compared to that of atop adsorption (see Fig. 2.10). Therefore, adsorption is stronger for 3-fold and bridge bonded sites. All of these reasons contribute to different back scattering properties for different adsorption sites and consequently their different $\Delta \mu$ spectra. Thus, different adsorption sites will cause $\Delta \mu$ signatures of the adsorbates to change both in energy and shape. The

$\Delta \mu$-XANES technique also can provide a measure of the relative coverage of adsorbates on the surface: the $\Delta \mu$ magnitudes of spectral features correlate with relative coverages. When the data quality is excellent, it is even possible to obtain quantitative estimates of the adsorbate coverage. 


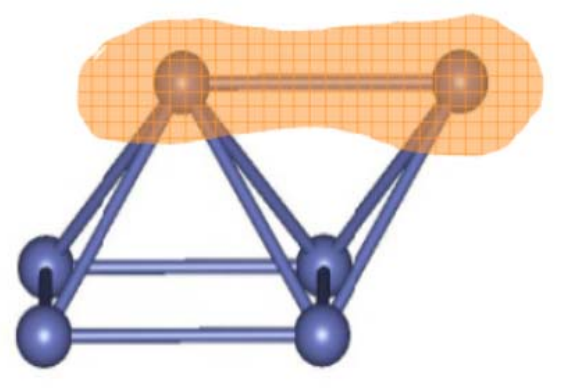

Plain Pt

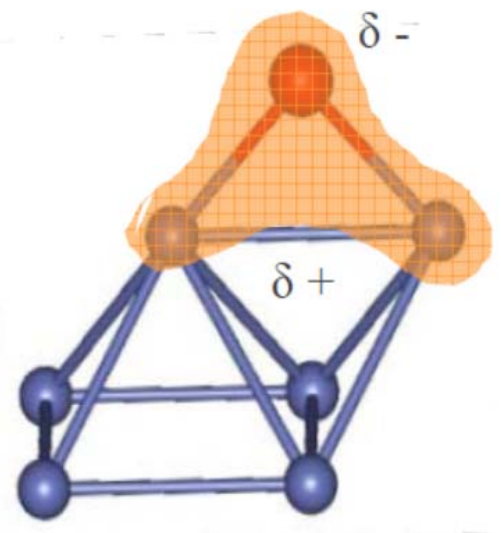

Pt cluster with adsorbate

Figure 2.10. Re-distribution of electronic charge on atoms in a cluster induced by adsorbate. The presence of adsorbates effectively reduces the distribution of electron density between the Pt atoms. 


\section{-Linear Combination Fitting}

Another XANES data analysis technique utilized in this dissertation is linear combination fitting (LCF). This is used for samples containing different species, such as a discharge

mixture of a battery electrode, as applied in chapters 5 and 6 of this dissertation. This analysis technique is based on the principle that the total absorption coefficient of a sample will be comprised of the sum of the absorption coefficients of all species present in a sample. It should be mentioned that this analysis is not limited to the XANES region and can be applied to derivative XANES and EXAFS regions as well. The mass absorption coefficient $\left(\mu_{\rho}\right)$ of a sample can be written as the sum of the absorption by each component present in the sample:

$$
\mu_{\rho}=\sum_{i} f_{i} \mu_{\rho i}
$$

where $f_{i}$ is the mass fraction of an element with mass absorption of $\mu_{\rho i}$. The absorption coefficient can also be written as a weighted sum of the absorption coefficients of the constituent elements of the sample, for instance in a cathode mixture comprised of $20 \%$ potassium ferrate $\left(\mathrm{K}_{2} \mathrm{FeO}_{4}\right)$ and $80 \% \quad \gamma$-ferric oxide $\left(\gamma-\mathrm{Fe}_{2} \mathrm{O}_{3}\right)$ the total absorption coefficient can be written as a weighted sum of the absorption coefficient of each of these two species. The linear combination fitting analysis utilizes the standard spectra of various possible compounds in a sample of unknown composition to model the samples' XAS spectrum. Athena computes the scaling factors required to apply the different spectra in a way that the addition of these scaled standard spectra will then represent the experimental spectrum the best. The mathematical model built by Athena to model the spectrum will be: 


$$
\text { Model }=\sum_{i} f_{i}\left(S T D_{i}\right)
$$

Here, the Model is the least-squares fit to the experimental sample spectrum (in a specific range), and $f_{i}$ is the scaling factor for each standard spectrum $\left(\mathrm{STD}_{\mathrm{i}}\right)$ in the sample. Again, in this type of analysis it is important to conduct a careful normalization step. The goodness of fits are represented with statistical parameters similar to those described for EXAFS fitting. 


\subsection{References}

(1) Teo, B. K. EXAFS Spectroscopy: Basic principles and data analysis. Springer-Verlag: New York, 1986; .

(2) Koningsberger, D. C.; Prins, R. Principles, techniques of EXAFS, SEXAFS and XANES. John Wiley \& Sons: New York, 1988; .

(3) Ramaker, D. E.; Koningsberger, D. C. Comment on "Effect of Hydrogen Adsorption on the X-Ray Absorption Spectra of Small Pt Clusters". Phys. Rev. Lett. 2002, 89, 139701.

(4) Teliska, M.; O'Grady, W. E.; Ramaker, D. E. Determination of O and OH Adsorption Sites and Coverage in Situ on Pt Electrodes from Pt L23 X-ray Absorption Spectroscopy. J. Phys. Chem. B 2005, 109, 8076.

(5) Scofield, J. H. Theoretical Photoionization Cross Sections from 1 to 1500 $\mathrm{keV}$ Lawrence Livermore National Laboratory Report UCRL 1973, 51326.

(6) Hubbell, J. R. Photon Cross Sections, Attenuation Coefficients, and Energy Absorption Coefficients from $10 \mathrm{keV}$ to $100 \mathrm{GeV}$. (1969). National Bureau of Standards, Washington DC. 1969, Report NSRDS-NBS 29.

(7) McMaster, W. H.; Del Grande, N. K.; Mallet, J. H.; Hubbell, J. H. Compilation of X-Ray Cross Sections. National Laboratory Report UCRL 1969, 50174 Section II Revision I.

(8) Henke, B. L.; Gullikson, E. M.; Davis, J. C. X-Ray Interactions: Photoabsorption, Scattering, Transmission, and Reflection at $E=50-30,000 \mathrm{eV}, Z=$ 1-92. Atomic Data and Nuclear Data Tables1993, 54, 181.

(9) Elam, W. T.; Ravel, B. D.; Sieber, J. R. A new atomic database for X-ray spectroscopic calculations. Rad. Phys. Chem. 2002, 63, 121.

(10) A. Shaltout, E., H.; Svagera, R. Update of photoelectric absorption coefficients in the tables of McMaster. X-ray Spectrometry 2005, 35, 52.

(11)Anonymoushttp://www4.nau.edu/microanalysis/microprobe/BulkAbsorp.ht ml. .

(12) Newville, M. IFEFFIT : interactive XAFS analysis and FEFF fitting. Journal of Synchrotron Radiation 2001, 8, 322. 
(13) Kelly, S. D.; Hesterberg, D.; Ravel, B. In Analysis of Soils and Minerals Using X-ray Absorption Spectroscopy; Ulery, A. L., Drees, L. R., Eds.; Methods of Soil Analysis - Part 5: Mineralogical Methods; Soil Science Society of America: Madison, WI, 2008; pp 367.

(14) Bevington, P. R.; Robinson, D. K. Data reduction and error analysis for the physical sciences.; McGraw-Hill: New York, 1992; Vol. 2nd ed.

(15) Stern, E. A.; Newville, M.; Ravel, B.; Yacoby, Y.; Haskel, D. The UWXAFS analysis package: Philosophy and details. Physica B 1995, $208 \& 209$, 117.

(16) Scott, F. J.; Mukerjee, S.; Ramaker, D. E. CO Coverage/Oxidation Correlated with PtRu Electrocatalyst Particle Morphology in 0.3 M Methanol by In Situ XAS. Journal of Electrochemical Soc. 2007, 154, A396.

(17) Roth, C.; Benker, N.; Buhrmester, T.; Mazurek, M.; Loster, M.; Fuess, H.; Koningsberger, D. C.; Ramaker, D. E. Determination of $\mathrm{O}[\mathrm{H}]$ and CO Coverage and Adsorption Sites on PtRu Electrodes in an Operating PEM Fuel Cell. J. Am. Chem. Soc. 2005, 127, 14607.

(18) Scott, F. J.; Mukerjee, S.; Ramaker, D. E. Contrast in Metal-Ligand Effects on $\mathrm{Pt}_{n} \mathrm{M}$ Electrocatalysts with $\mathrm{M}$ Equal Ru vs Mo and Sn As Exhibited by in Situ XANES and EXAFS Measurements in Methanol. J. Phys. Chem. C 2010, 114, 442.

(19) Savitzky, A.; Golay, M. J. E. Smoothing and Differentiation of Data by Simplified Least Squares Procedures.. Anal. Chem. 1964, 36, 1627.

(20) Janin, E.; von Schenck, H.; Gothelid, M.; Karlsson, U. O.; Svensson, M. Bridge-bonded atomic oxygen on Pt(110) . Phys. Rev. B 2000, 61, 13144.

(21) Stern, E. A.; Heald, S. M. X-ray filter assembly for fluorescence measurements of X-ray absorption file structure. Rev. Sci. Instrum. 1979, 50, 1579.

(22) Ravel, B.; Newville, M. ATHENA, ARTEMIS, HEPHAESTUS: data analysis for X-ray absorption spectroscopy using IFEFFIT. J. Synchrotron Radiation 2005, 12, 537.

(23) Ravel, B. ATOMS: crystallography for the X-ray absorption spectroscopist. Journal of Synchrotron Radiation 2001, 8, 314. 
(24) Hennig, C.; Reich, T.; Funke, H.; Rossberg, A.; Rutsch, M.; Bernhard, G. EXAFS as a tool for bond-length determination in the environment of heavy atoms. J. Synchrotron Radiation2001, 8, 695.

(25) Maeda, H. Accurate Bond Length Determination by EXAFS Method. $J$. Phys. Soc. Jpn. 1987, 56, 2777.

(26) Arcovito, A.; Benfatto, M.; Cianci, M.; Hasnain, S. S.; Nienhaus, K.; Nienhaus, G. U.; Savino, C.; Strange, R. W.; Vallone, B.; Della Longa, S. X-ray structure analysis of a metalloprotein with enhanced active-site resolution using in situ x-ray absorption near edge structure spectroscopy. Proc. Natl. Acd. Sci. 2007, 104,6211 .

(27) Randaccio, L.; Geremia, S. Comparison of the Coordination Distances Derived by Extended X-ray Absorption Fine Structure and X-ray Crystallography in a Vitamin B12 Model. Organometallics1997, 16, 4951.

(28) Levina, A.; Armstrong, R. S.; Lay, P. A. Three-dimensional structure determination using multiple-scattering analysis of XAFS: applications to metalloproteins and coordination chemistry. Coordination Chem. Rev. 2005, 249, 141.

(29) Dalba, G.; Fornasini, P. EXAFS Debye-Waller Factor and Thermal Vibrations of Crystals. J. Synchrotron Radiation 1997, 4, 243. Bunker, G. Tutorials

on XAFS. http://gbxafs.iit.edu/training/tutorials.html. .

(31) Dirac, P. A. M. The Principles of Quantum Mechanics; Oxford University Press: 1982; .

(32) Sayers, D.; Lytle, F.; Stern, E. New Technique for Investigating Noncrystalline Structures: Fourier Analysis of the Extended X-Ray Absorption Fine Structure. Phys. Rev. Lett. 1971, 27, 1204.

(33) Teliska, M.; O'Grady, W. E.; Ramaker, D. E. Determination of $\mathrm{H}$ Adsorption Sites on $\mathrm{Pt} / \mathrm{C}$ Electrodes in $\mathrm{HClO} 4$ from Pt L23 X-ray Absorption Spectroscopy. J. Phys. Chem. B 2004, 108, 2333.

(34) Zabinsky; S.I.; Rehr, J.J.; Ankudinov, A.; Albers, R.C.; Eller, M.J. Multiple-scattering Calculations of X-ray-absorption Spectra. Phys. Rev. B 1995, 52, 2995. 
(35) Roth, C.; Benker, N.; Buhrmester, M.; Mazurek, M.; Loster, M.; Fuess, H.; Koningsberger, D.; Ramaker, D.E.; Determination of $\mathrm{O}[\mathrm{H}]$ and $\mathrm{CO}$ Coverage and Adsorption Sites on PtRu Electrodes in an Operating PEM Fuel Cell, J.AM. Soc. 2005, $127,14607$.

(36) Shyam, B.; Understanding the Poisoning, Aging and Degradation of Lowtemperature Fuel CellElectrocatalysts using in situ X-ray Absorption Spectroscopy, 2010, The George Washington University, Washington, DC. 


\section{Chapter 3: In-Situ X-ray Absorption Spectroscopy Studies on Adsorption of Bromide and Iodide on Platinum}

\subsection{Introduction:}

Polymer electrolyte membrane fuel cells (PEMFC) have been extensively studied in recent years due in part to the increased desired for developing more efficient energy conversion devices, and the use of alternate fuels. PEMFCs have been utilized as energy conversion devices both in mobile applications such electric cars as well as for stationary power generation. Polycrystalline platinum nanoparticles are the most commonly used electrocatalyst in these PEMFCs. Platinum (and platinum alloys) show the highest activity towards the four electron reduction of $\mathrm{O}_{2}$ to water in acidic environments. The oxygen reduction reaction (ORR) over Pt however exhibits slow kinetics compared to the very fast kinetic behavior of the hydrogen oxidation reaction. There is a $400 \mathrm{mV}$ overpotential loss for $\mathrm{O}_{2}$ reduction on the cathode side, which is about ten times higher than that for $\mathrm{H}_{2}$ oxidation at the anode. ${ }^{1,2}$

The undesirable high overpotential is due not only to the slow ORR kinetics, but also anion adsorption such as $\mathrm{OH}^{-}$coming from water activation. The already sluggish ORR kinetics can further be affected by many impurities, even in trace amounts. The presence of any kind of impurities can adversely affect the ORR activity, alter reaction pathways, block adsorption sites and affect product distribution. For instance it has been shown that the presence of organic residues can produce mixed potentials at the cathode that can then lead to loss in power output. ${ }^{3}$ 
One common procedure to synthesize platinum and platinum alloy particles supported on high surface area carbon substrates involves the utilization of halide containing reactants (e.g. $\mathrm{Cl}^{-}$containing salts). ${ }^{5,6,7}$ Additionally, halides are likely to be found in the humidified anode feed streams of PEMFCs or in the cathode air feed; the latter particularly containing $\mathrm{Cl}^{-}$and other halides in the "salty" air of oceanic environments. Thus, there is a high possibility of finding traces of halide contaminant in the electrocatalyst. The presence of halide anions can act as a poison and interfere with the pathway for ORR activation. Halide anions can compete with $\mathrm{O}_{2}$ for adsorption on catalyst surfaces; this competition can potentially decrease the ORR activity, and alter the reaction pathway to undesired product formation, such as $\mathrm{H}_{2} \mathrm{O}_{2}$ byproduct. It has been shown that the presence of $\mathrm{H}_{2} \mathrm{O}_{2}$ can result in poor use of the cathode gas feed, which in turn can cause enhancements in parasitic compressor power. It can also increase the formation of hydroxyl and peroxy-hydroxyl free radicals that can attach to the PEM and decrease the durability of the membrane. ${ }^{8,9}$

Over the years, many studies have been conducted on the nature and mobility of halide adsorption on Pt. However, there are different views on the comparative reversibility of halide adsorption. Voltammetric studies of halide adsorption on a smooth Pt surface in $\mathrm{HClO}_{4}$ environment has shown that the adsorption strength of these anions vary in the order of $\mathrm{Cl}^{-}<\mathrm{Br}-<\mathrm{I}^{-} .{ }^{10}$ Voltammetry experiments also show that $\mathrm{Cl}^{-}$and $\mathrm{Br}^{-}$adsorption on $\operatorname{Pt}(111)$, even at $\mu \mathrm{M}$ concentrations, can affect the $\mathrm{H}_{\text {upd }}$ region as indicated by the appearance of a distinct but reversible peak in the cyclic voltammetry plots. This peak suggests a coupled $\mathrm{H}_{\text {and }} \mathrm{Cl}^{-}$adsorption/desorption reaction. Further, it has been shown that at low $\mathrm{pH}$ and high $\mathrm{Cl}^{-}$concentrations, the chloride adsorption competes even more 
aggressively with the hydrogen adsorption. In general, any change of the CV peaks in the $\mathrm{H}_{\text {upd }}$ region due to the presence of halides, either in potential or magnitude, proves the competition between halide and $\mathrm{H}^{+}$adsorption on a positively charged Pt surface. ${ }^{11}$ In the presence of $\mathrm{Cl}^{-}$, the "butterfly" region, lying just above the $\mathrm{H}_{\text {upd }}$ region in the $\mathrm{CV}$, is suppressed, and the sharp CV peak observed at higher potentials due to adsorption of oxygenated species is shifted upward. The shift observed in this anodic peak shows that halide anions also suppress $\mathrm{Pt}-\mathrm{O}[\mathrm{H}]$ formation. ${ }^{10-12}$

In our previous work ${ }^{13}$, the adsorption of $\mathrm{Cl}^{-}$on various $\mathrm{Pt}$ sites of $\mathrm{Pt} / \mathrm{C}$ supported catalysts was studied using electrochemical measurements (CV and RDE) and in-situ x-ray absorption spectroscopy (XAS). At $\mathrm{Cl}^{-}$concentrations of 1 and $10 \mathrm{mM}, \mathrm{Cl}^{-}$was adsorbed mostly in 3 -fold sites. However, at potentials between 0.4 to $0.7 \mathrm{~V}$ vs. RHE a compressed adlayer of $\mathrm{Cl}^{-}$was formed on the surface, where some $\mathrm{Cl}^{-}$anions are forced into atop and bridged positions. Further, it was shown that formation of the $\mathrm{Cl}^{-}$adlayer increased the overpoential by $150-200 \mathrm{mV}$ for water activation at the $\mathrm{Pt}(111)$ surface. The ORR pathway was also affected.

In this work, the adsorption of bromide and iodide on the Pt(111) surface will be investigated through electrochemical characterization and XAS studies. Specifically, this work is focused on halide adsorption in the double-layer and $\mathrm{Pt}-\mathrm{O}[\mathrm{H}]$ formation regions of the $\mathrm{CV}$, and to investigate the effects of halide adsorption on the ORR activity. A variety of electrochemical and spectroscopic techniques have previously been employed in studying the effects of halide poisoning on the ORR, namely: cyclic voltammetry (CV), chrono methods ( amperometry, coulombmetry, etc.), rotating ring disk electrode measurements 
(RRDE), Auger electron spectroscopy, low-energy electron diffraction (LEED), second harmonic generation (SHG), and surface x-ray scattering (SXS). ${ }^{14-17}$ X-ray scattering spectroscopy (SXS) studies on the adsorption of chloride, bromide and iodide on $\operatorname{Pt}(111)$ revealed interesting information on the structure of the adlayer formed by these ions: iodide is known to form two commensurate adlayer structures, a $(\sqrt{7} \times \sqrt{7})$ R19.1 phase and a hexagonal $(3 \times 3)$ phase which coexist on the surface ${ }^{32-35}$. It has been proposed that the ( $\sqrt{7} \times \sqrt{7}$ ) phase contains hexagonal I' geometries with 3-fold and atop adsorption sites and the $(3 \times 3)$ phase shows a combination of bridge bonded and atop sites. Bromide, on the other hand, forms a series of high-order commensurate structures that are poorly ordered. ${ }^{16}$

All these techniques, while providing very useful insight into the effects of halide poisoning on the ORR, don't provide a complete picture concerning the adsorption of halides on Pt in operando conditions. Most of the spectroscopic techniques utilized so far to study halide adsorption require ultra high vacuum conditions and therefore are not suitable for studies in a real working fuel cell environment. Further, most of these studies were conducted on single crystals, rather than on polycrystalline or supported catalysts and therefore again are in circumstances far removed from real commercial electrocatalyst.

In this work, in-situ X-ray absorption spectroscopy (XAS) combined with electrochemical characterization is utilized to study the adsorption of bromide and iodide on supported Pt/C. XAS is an element specific technique and can be utilized for in-situ and operando measurements with no need of ultra high vacuum, such as that required by electron spectroscopic techniques. Analysis of both X-ray absorption near edge (XANES) and extended fine structure (EXASF) regions of the spectrum can simultaneously provide 
electronic and adsorbate coverage information and structural and morphological information. Here, the $\Delta \mu$ XANES analysis technique is applied to follow formation of the iodide and bromide adlayer on supported $\mathrm{Pt} / \mathrm{C}$ catalysts as the in-situ cell is electrochemically cycled at various potentials. These results are compared to similar results we obtained previously for chloride ${ }^{13}$, as well as to other results in the literature.

\subsection{Experimental:}

\subsubsection{Electrochemical Measurements:}

Details of the experimental procedures were described in detail previously. ${ }^{13}$ Briefly, a polished glassy carbon rotating disk electrode (RDE) ( Pine Instrument Co. ) was painted with two aliquots of $30 \mathrm{wt} \% \mathrm{Pt} / \mathrm{C}$ inks (Vulcan XC72 from E-TEK). These inks were prepared by combining $10 \mathrm{mg}$ catalysts, $5 \mathrm{ml}$ deionized water (18.2 M , Millipore MilliQ system), $5 \mathrm{~mL}$ 2-propanol (HPLC grade, Aldrich), and $40 \mu \mathrm{L} 5 \mathrm{wt} \%$ Nafion solution (Aldrich). The painted RDEs were then allowed to air dry. The final Pt loading on the glossy carbon $\mathrm{RDE}$ was $14 \mu \mathrm{g} \mathrm{cm} \mathrm{cm}^{-2}$. The $\mathrm{CV}$ experiments were conducted at room temperature in $1 \mathrm{M} \mathrm{HClO}_{4}\left(\mathrm{GFS}\right.$ Chemicals, doubly distilled), $1 \mathrm{M} \mathrm{HClO}_{4}+\mathrm{nM} \mathrm{KX}\left(\mathrm{n}=10^{-}\right.$ ${ }^{3}, 10^{-2}$ and $\mathrm{X}=\mathrm{Cl}^{-}, \mathrm{Br}^{-}, \mathrm{I}^{-}$) under an $\mathrm{Ar}$ (Med-Tech Gasses) atmosphere. The above electrolytes were purged with ultra high purity $\mathrm{O}_{2}$ for oxygen reduction experiments. An Autolab potentiostat (model PGSTAT30, Ecochemie-Brinkmann) was utilized for all data collection. For all the experiments, the reference electrode used was a sealed RHE filled with $1 \mathrm{M} \mathrm{HClO}_{4}$, and a high surface area Pt mesh (Alfa Aeser) was used as counter electrode. The poisoning effects of halide anions make $\mathrm{H}_{\text {upd }}$ adsorption as observed in by 
the current in a CV useless for surface area measurements, thus all current densities reported in this text refer to geometric surface area. The average particle size of these electrocatalysts was in the range $1-2 \mathrm{~nm}$.

\subsubsection{In Situ XAS Data Collection:}

A previously reported, an in-situ cell design was utilized for all XAS experiments. ${ }^{18}$ The working electrode (WE) was $30 \mathrm{wt} \% \mathrm{Pt} / \mathrm{VXC} 72$ (E-TEK), the counter electrode (CE) consisted of an acid washed $\left(0.5 \mathrm{M} \mathrm{H}_{2} \mathrm{SO}_{4}\right)$ Grafoil ${ }^{\circledR}$ and an RHE served as reference electrode. A piece of Nafion ${ }^{1} 12$ (DuPont) polymer electrolyte membrane separated the WE and CE. The cell was flooded with $1 \mathrm{M} \mathrm{HClO}_{4}+\mathrm{nM} \mathrm{KX}$ (where $\mathrm{n}=10^{-3}$ or $10^{-2} \mathrm{M}$ and $\mathrm{X}=\mathrm{Cl}, \mathrm{Br}, \mathrm{I}$ ). A Au wire ( $99.999 \%$ Alfa-Aesar) was used as a current collector, pressed mechanically on the back side of the electrode; it was carefully placed so as not to be exposed to the X-ray beam. The catalyst suspensions of $30 \mathrm{wt} \% \mathrm{Pt} / \mathrm{VXC} 72,1: 1 \mathrm{de}-$ ionized $\mathrm{H}_{2} \mathrm{O} / 2$-propanol mixture and 95:5 mixture of catalyst (wt)/ $5 \mathrm{wt} \%$ Nafion solution were prepared and then hand painted on a commercially available carbon cloth (Zoltek Corp.) with a loading of ca. $5 \mathrm{mg} \mathrm{cm}^{-2}$. These electrodes possessed an x-ray absorption cross section of about 1 and the total geometric area of the Pt WE used in cell was about 5 $\mathrm{cm}^{2}$. The electrodes were then vacuum impregnated for 1 hour in $1 \mathrm{M} \mathrm{HClO}_{4}$ prior to cell assembly to ensure that they were completely wet. The WE was first activated by potential cycling $\left(0.05 \mathrm{~V}\right.$ to $1.2 \mathrm{~V}$ vs. RHE at $\left.10 \mathrm{mVs}^{-1}\right)$ in clean $1 \mathrm{M} \mathrm{HClO}_{4}$, followed by removal of clean electrolyte and replacement with $1 \mathrm{M} \mathrm{HClO}_{4}+\mathrm{nM} \mathrm{KX}\left(\mathrm{n}=0,10^{-3}, 10^{-2}\right)$ with a syringe. Extended x-ray absorption fine structure (EXAFS) scans were taken $(-250 \mathrm{eV}$ to $18 \mathrm{~K}$ ) while the $\mathrm{WE}$ was held at a fixed static potential at various points during the anodic 101 
sweep. Between each EXAFS scan, the potential was cycled to completely remove Pt-O traces. To provide data as clean reference scans and as $\mathrm{H}_{2} \mathrm{O}$ activation references, a set of spectra were obtained in "clean" $1 \mathrm{M} \mathrm{HClO}_{4}$.

All XAS data were collected at beam line X11-B (National Synchrotron Light Source, Brookhaven National Laboratory, Upton, NY). A Si(111) monochromator was detuned by $40 \%$ to reject higher harmonics. All data were taken in transmission mode, where the sample was placed between the $\mathrm{I}_{0}$ and $\mathrm{I}_{\mathrm{t}}$ detectors and a Pt foil was positioned between $\mathrm{I}_{0}$ and $\mathrm{I}_{\text {ref }}$ to account for possible drift in beam energy over time. The ionization detectors ( $\mathrm{I}_{0}$, $\mathrm{I}_{\mathrm{t}}$ and $\left.\mathrm{I}_{\mathrm{ref}}\right)$ were operating with a nominal Nitrogen/Argon gas mixture to allow $\sim 10 \%$ photon absorption in $\mathrm{I}_{0}$ and $70 \%$ in $\mathrm{I}_{\mathrm{t}}$.

\subsection{3. $\Delta \mu$-XANES Technique:}

The IFEFFIT suite $^{19}$ (Version 1.2.11 IFEFFIT Copyright 2008, Mathew Newville, University of Chicago, http://cars9.uchicago.edu/ifeffit) was utilized for data analysis, background subtraction (AutoBK) ${ }^{20}$ and normalization. The $\Delta \mu$ analysis technique has

been described in detail previously. ${ }^{21-24}$ In brief, XAS spectra were first calibrated to the edge energy (11564 eV, Pt $\mathrm{L}_{3}$ edge). All spectra were then aligned to a reference scan of Pt foil to correct for beam energy drift over time. All energy shift corrections applied to the reference foils are automatically applied to the respective sample scans. Careful energy alignment of all spectra is a must to ensure that the atomic contribution in each $\mu$ cancels out when taking the $\Delta \mu$ difference. The post-edge normalization procedure (20-300 eV above the edge, for $\Delta \mu$-XANES analysis) is applied to all sample spectra. The 
normalization procedure, using a cubic spline function (AUTOBK) ${ }^{20}$ contained within the IFEFFIT package, gives the $\mu$ data on a per-atom basis. Difference spectra are then constructed as:

$\Delta \mu=\mu(\mathrm{V}, \mathrm{x} \mathrm{mM} \mathrm{KX})-\mu(0.54 \mathrm{~V}, 0.0 \mathrm{M} \mathrm{KX})$

where $\mu(\mathrm{V}, \mathrm{x} \mathrm{mM} \mathrm{KX)}$ is the absorption spectrum of the sample at various potentials and $\mathrm{KX}$ concentrations and $\mu(0.54 \mathrm{~V}, 0.0 \mathrm{M} \mathrm{KX})$ is the absorption signal at $0.54 \mathrm{~V}$ in the "clean" cases (i.e., without any $\mathrm{KX}$ present). The spectrum collected at $0.54 \mathrm{~V}$ is selected as the reference since it is assumed that at $0.54 \mathrm{~V}$ the Pt surface is cleanest (i.e: water activation is at a minimum, so neither $\mathrm{H}$ nor $\mathrm{O}$ is prevalent on the surface) and in the absence of KX no halides are on the surface.

The experimental $\Delta \mu$ are then compared to theoretical curves $\left(\Delta \mu_{\mathrm{t}}\right)$ constructed using the FEFF 8.0 code. The theoretical signature is calculated as:

$$
\Delta \mu_{\mathrm{t}}=\mu\left(\mathrm{X} / \mathrm{Pt}_{6}\right)-\mu\left(\mathrm{Pt}_{6}\right)
$$

where $\mathrm{X}$ is a halide anion $\left(\mathrm{F}^{-}, \mathrm{Cl}^{-}, \mathrm{Br}^{-}, \mathrm{I}^{-}\right)$or $\mathrm{O}$ in a specific binding site with respect to the absorbing Pt atom. Here, $\mathrm{Pt}_{6}$ is a six-Pt cluster with a Pt-Pt bond distance of $2.77 \mathrm{~A}$ as

described by Janin et al. ${ }^{25}$ The theoretical $\Delta \mu$ are usually shifted by $1-5 \mathrm{eV}$ and need to be scaled by a multiplication factor for optimum comparison with experimental data.

\subsection{Results and Discussion:}

\subsubsection{Electrochemical Characterization:}

Figure 3.1 shows ORR sweeps for 1 and $10 \mathrm{mM} \mathrm{KBr}$. For an initial concentration of $1 \mathrm{mM}$ of $\mathrm{Br}^{-}$, an overpotential of $200 \mathrm{mV}$ is observed relative to when no $\mathrm{KBr}$ is present; 
increasing the concentration to $10 \mathrm{mM}$ adds another $150 \mathrm{mV}$ of overpotential. Further, there is no limiting current observed above $0.2 \mathrm{~V}$ for either concentration of $\mathrm{KBr}$. This illustrates that compared to previous results for $\mathrm{Cl}^{-17,26}, \mathrm{Br}^{-}$seems to be more effectively blocking surface sites required for the ORR, perhaps because $\mathrm{Br}^{-}$is more strongly adsorbed to the $\mathrm{Pt}$ surface or simply because it is a bigger ion. Figure 3.2a shows $\mathrm{CV}$ profiles for $\mathrm{Pt}$ in the indicated concentrations of $\mathrm{KBr}$. At a $\mathrm{Br}^{-}$concentration of $10^{-4} \mathrm{M}$, slight variations are observed in the $\mathrm{H}_{\text {upd }}$ region, a very subtle increase in the double-layer region is seen, and Pt-O formation appears to nearly progress as normal. Increasing the $\mathrm{Br}^{-}$ concentration to 1 and $10 \mathrm{mM}$ almost entirely suppresses the Pt-O formation peak. At these concentrations a $\mathrm{Br}_{2}$ evolution peak starts at $1.0 \mathrm{~V}$. Increasing the $\mathrm{Br}$ - concentration to $100 \mathrm{mM}$, the $\mathrm{Br}_{2}$ formation peak at potentials larger than $1.0 \mathrm{~V}$ overwhelms all three regions and significant changes are observed. The current density at this concentration experiences a 10 -fold increase compared to the $10 \mathrm{mM} \mathrm{Br}^{-}$results, resulting from the dramatic increase in $\mathrm{Br}_{2}$ evolution, and suggests an exponential relationship between $\mathrm{Br}^{-}$ concentration and $\mathrm{Br}_{2}$ evolution. Further, based on previous results in the literature ${ }^{26} \mathrm{Br}^{-}$is known to form a highly ordered commensurate (HOC) in-plane adlayer on the Pt surface. 


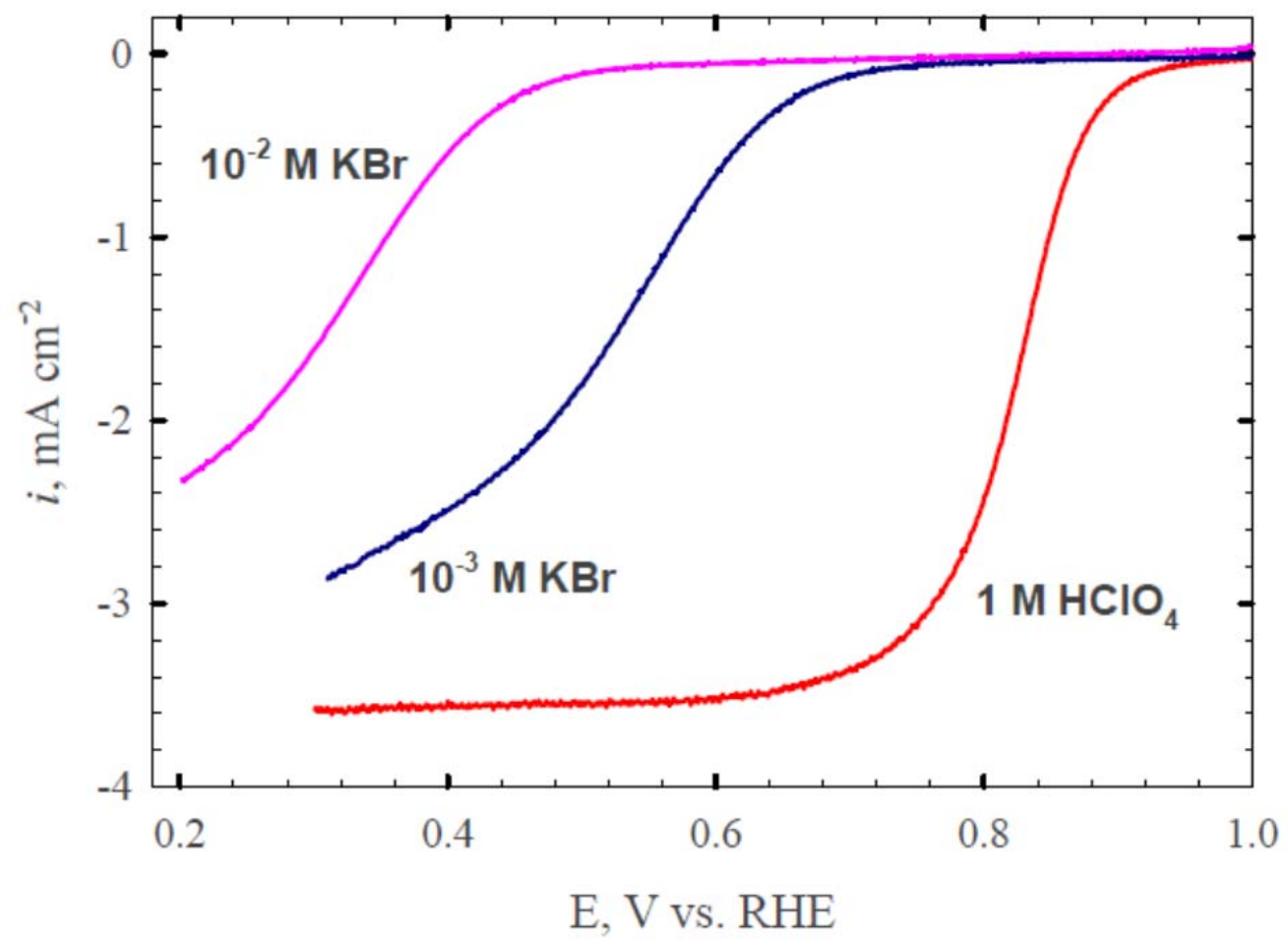

Figure 3.1. ORR polarization curves for $30 \mathrm{wt} . \% \mathrm{Pt} / \mathrm{C}$ the indicated concentrations of $\mathrm{KBr}$ in $1 \mathrm{M} \mathrm{HClO}_{4}$ taken at $900 \mathrm{RPM}$ at $20 \mathrm{C}$. In each case the electrolyte was saturated with $\mathrm{O}_{2}$. 
The poisoning effects are even larger with $\mathrm{I}^{-}$, as illustrated in Figure 3.2b. At 10 and $100 \mu \mathrm{M}$ concentrations of $\mathrm{KI}$, significant poisoning effects occur in all three regions. The $\mathrm{H}_{\text {upd }}$ region is severely blocked by adsorbed I-: indeed the $\mathrm{H}_{\text {upd }}$ peak entirely disappears in the presence of $\mathrm{KI}$ resulting in a single peak at $0 \mathrm{~V}$ and this diminishes in intensity with increase of KI. Since it is well known ${ }^{36}$ that the normal $\mathrm{H}_{\text {upd }}$ peaks results from 3 -fold fcc $\mathrm{H}$ adsorption, and atop $\mathrm{H}$ occurs around $0 \mathrm{~V}$, this strongly suggest that the $\mathrm{I}^{-}$entirely blocks the fcc sites, allowing $\mathrm{H}$ adsorption only in the atop sites. A small peak in the Pt-O formation region is observed in the presence of $\mathrm{I}^{-}$below $100 \mu \mathrm{M} \mathrm{KI}$, but it is not easy to discern if this peak is due to Pt-O formation or $\mathrm{I}_{3}^{-}$formation since at $1 \mathrm{mM} \mathrm{KI}$ a large $\mathrm{I}_{3}{ }^{-}$ peak is evident falling very near the same potential as the normal Pt-O formation peak.

At $1 \mathrm{mM}$ concentration the redox couple is easily observed, which can be attributed to these simultaneous reactions:

$50 \mathrm{mVs}^{-1}$. Current densities reflect the geometric surface area of a $5.56 \mathrm{~mm}$ glasssy carbon disk.

$\mathrm{I}_{2}+2 \mathrm{e}^{-} \longrightarrow 2 \mathrm{I}^{-}$

$\mathrm{I}_{3}^{-}+2 \mathrm{e}^{-} \longrightarrow 3 \mathrm{I}^{-}$ 
a)

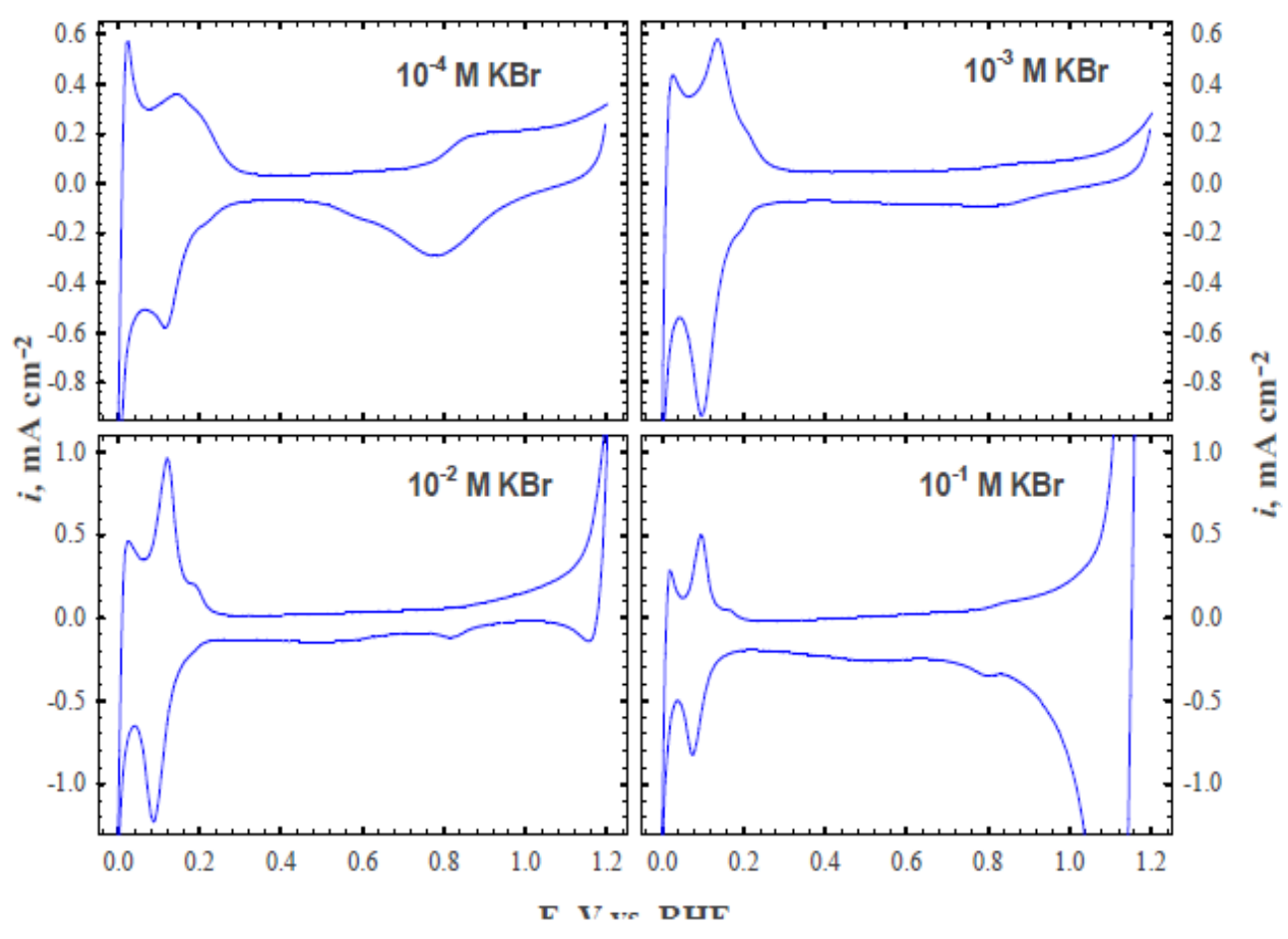

b)

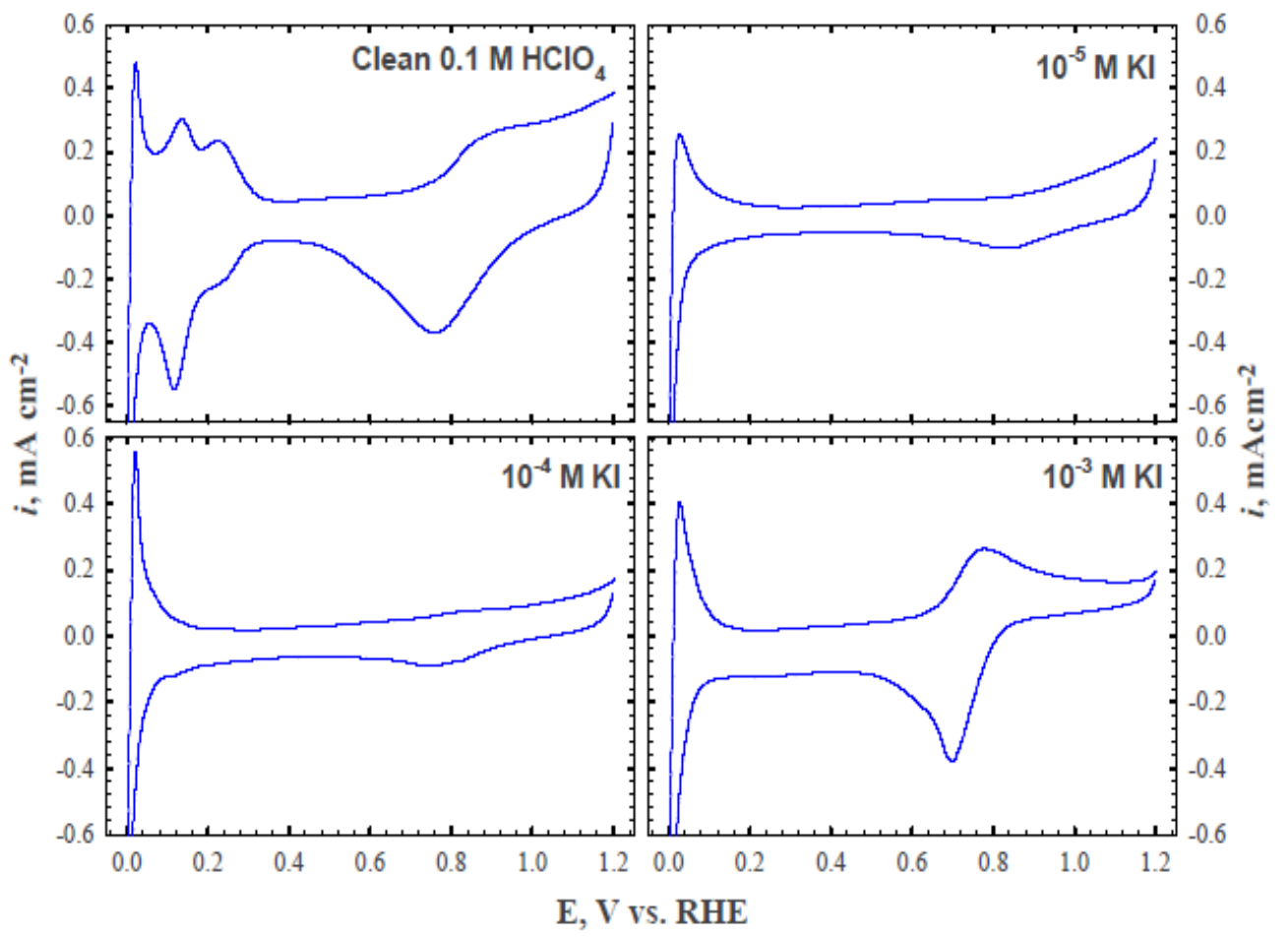

Figure 3.2 Cyclic voltammograms of 30 wt. \% Pt/C collected using the indicated concentrations of a) $\mathrm{KBr}$ and b) $\mathrm{KI}$. The scans were collected at $20^{\circ} \mathrm{C}$. 


\subsection{2. $\Delta \mu$ Results}

Our previous paper ${ }^{13}$ concentrated on the site specific adsorption (and resultant poisoning of the ORR) of chloride on Pt catalysts in acidic media. The in-situ $\Delta \mu$ XANES technique clearly revealed the chloride coverage and the adsorption site. Chloride adsorption on $\mathrm{Pt}$ was shown to be in equilibrium at all potentials:

$\mathrm{Cl}^{-}+\mathrm{Pt} \longrightarrow \mathrm{Cl}^{-} / \mathrm{Pt}$

The experimental $\Delta \mu$ signature and comparison with FEFF8 calculated theoretical $\Delta \mu$ signatures indicated that the $\mathrm{Cl}^{-}$adsorbs in a site specific n-fold site on $\operatorname{Pt}(111)$ faces. The theoretical results in Figure 3.3a for $\mathrm{Cl}^{-}$shows that the $\Delta \mu$ signature is significantly different from the others only for the atop case because of the absence of the negative feature around $0 \mathrm{eV}$. For the other multiply coordinated cases (bridged, fcc, and hcp) the negative feature around $0 \mathrm{eV}$ is present with only slightly varying size. Thus we can distinguish via the $\Delta \mu$ signature only whether the $\mathrm{Cl}$ is in an atop site vs $\mathrm{n}$-fold coordination.

Figure 3.5a shows $\Delta \mu$ spectra for $\mathrm{H}_{2} \mathrm{O}$ activation on $\mathrm{Pt}$ in $1 \mathrm{mM} \mathrm{HClO} 4$ at various potentials $\left(0.24 \mathrm{~V}\right.$ to $1.15 \mathrm{~V}$ vs. RHE). Below $0.24 \mathrm{~V}$ the well-known signature ${ }^{27},{ }^{36}$ for $\mathrm{H}_{\text {upd }}$ is observed, having a large negative feature near $0 \mathrm{eV}$, and positive feature around 8 $\mathrm{eV}$. As the potential increases to $0.7 \mathrm{~V}$ vs. RHE a positive peak near the absorption edge energy arises due to $\mathrm{OH}$ adsorption. As the potential is increased from $0.7 \mathrm{~V}$ to $1.15 \mathrm{~V}$ this peak shifts to higher energies and its magnitude also increases due to $\mathrm{O}$ adsorption. This shift in energy and increase in peak magnitude corresponds to the transition from $\mathrm{OH}$ atop 

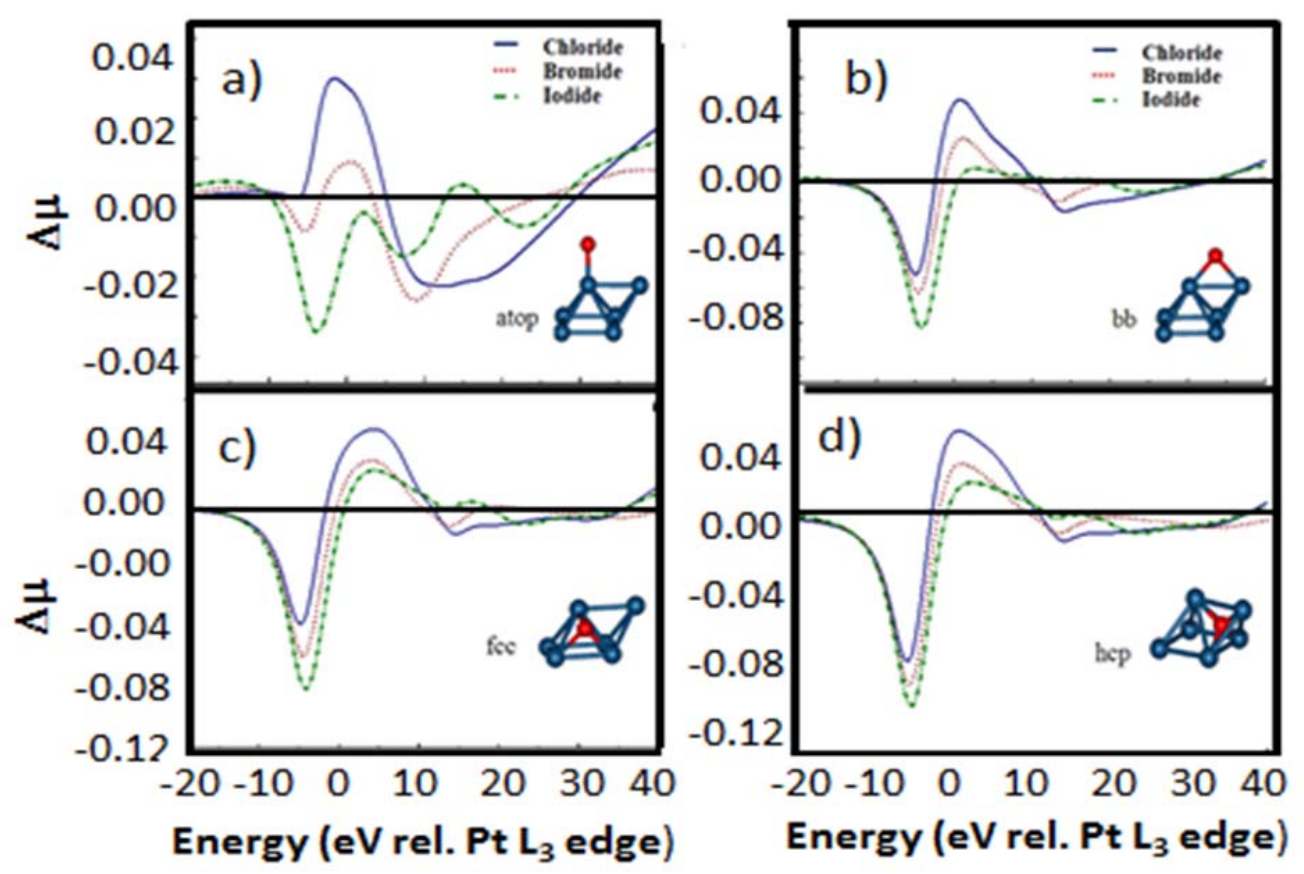

Figure 3.3 The theoretical $\Delta \mu$ signatures for each adsorbed halide on the surface of $\mathrm{Pt}_{6}$ cluster in four possible adsorption sties: a)atop, b) bridged, c) 3-fold (fcc) and d) hexagonal closed pack (hcp). $\Delta \mu_{\mathrm{t}}=\mu\left(\mathrm{Pt}_{6}-\mathrm{X}\right)-\mu\left(\mathrm{Pt}_{6}\right)$; where $\mathrm{X}$ is $\mathrm{Cl}^{-}, \mathrm{Br}^{-}$and $\mathrm{I}^{-}$. The Pt-X bond distances are calculated based on atomic radii, and are: $2.35 \mathrm{~A}^{\circ}, 2.50 \mathrm{~A}^{\circ}$ and $2.75 \mathrm{~A}^{\circ}$ for $\mathrm{Pt}-\mathrm{Cl}, \mathrm{Pt}-\mathrm{Br}$ and $\mathrm{Pt}-\mathrm{I}$ respectively. 


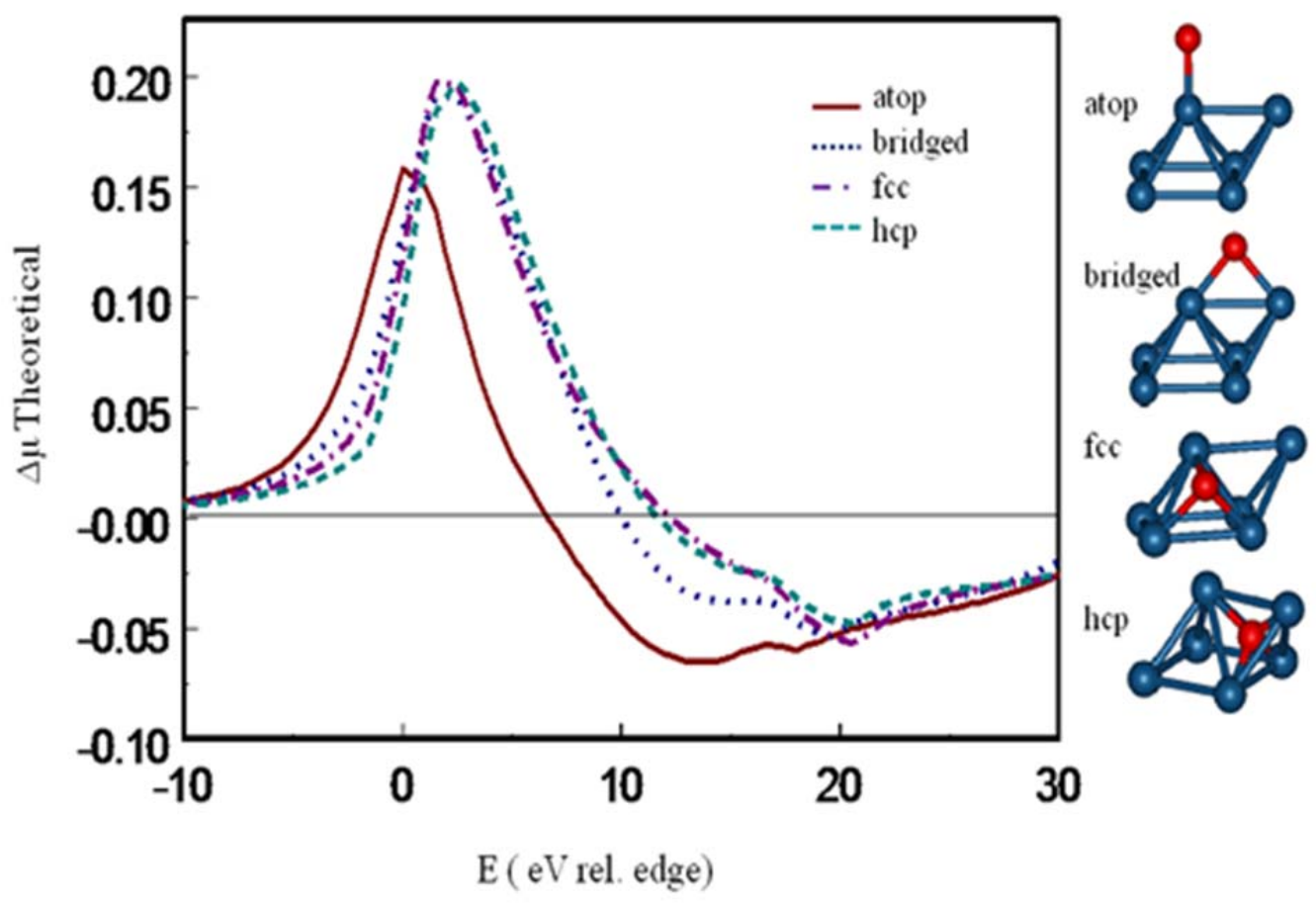

Figure 3.4 Theoretical $\Delta \mu$ signatures for Oxygen adsorption on $\mathrm{Pt}_{6}$ cluster in atop, bridged, fcc and hep configurations. 


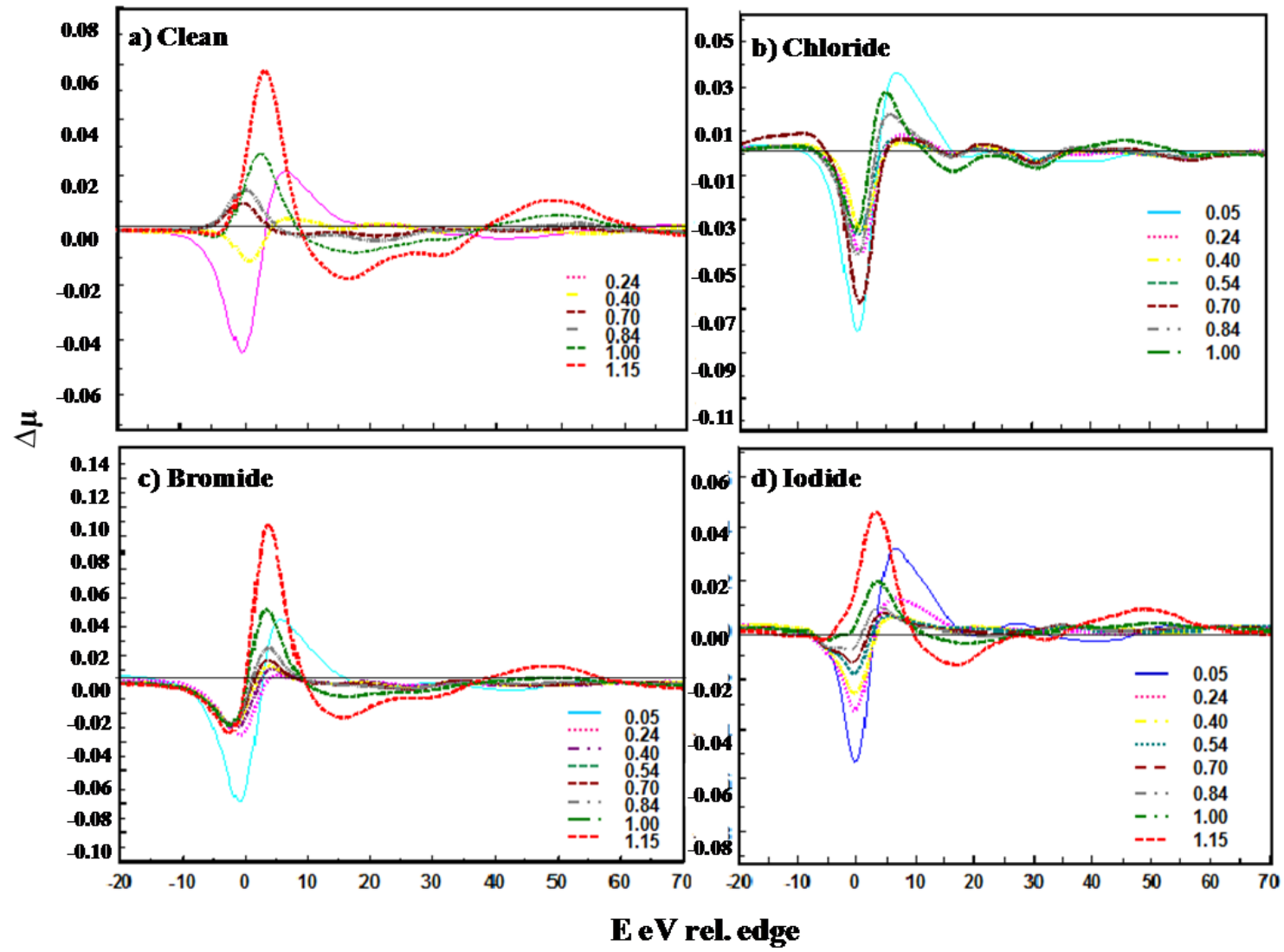

Figure $3.5 \mathrm{Pt} \mathrm{L}_{3}$ edge $\Delta \mu=\mu(\mathrm{V}$, halide $)-\mu(0.54 \mathrm{~V}$ clean $)$ spectra for $30 \mathrm{wt} \% \mathrm{Pt} / \mathrm{C}$ in $1 \mathrm{M}$ $\mathrm{HClO} 4$ and $10 \mathrm{mM}$ of b) chloride, c) bromide and d)iodide. 5a illustrates $\Delta \mu$ plots in a $1 \mathrm{M}$ $\mathrm{HClO} 4$ electrolyte with no added halide. 
to $\mathrm{n}$-fold $\mathrm{O}$ adsorption as indicated by Fig. 3.4, where the theoretical $\Delta \mu$ lines shapes for $\mathrm{OH} / \mathrm{O}$ adsorption on $\mathrm{Pt}$ are shown as previously reported by Teliska et. al. ${ }^{27}$

Because of the low scattering properties of $\mathrm{H}$, it does not contribute significantly to the $\Delta \mu$ for $\mathrm{OH}$, but DFT calculations ${ }^{25,28,29}$ have previously shown that $\mathrm{OH}$ prefers atop adsorption and $\mathrm{O}$ prefers $\mathrm{n}$-fold. Therefore, $\Delta \mu$ can indirectly distinguish between these two types of species through their different atop vs n-fold adsorption sites.

The physical basis for the difference between these signatures has been discussed in detail previously. ${ }^{27}$ It mainly arises because the Pt-O scattering does not change significantly with change in binding site, however, the Pt-Pt does. Once the O moves to any of the multi-coordinated n-fold sites, the Pt-Pt bonds immediately below weaken resulting in reduced Pt-Pt scattering; hence shifting the $\Delta \mu$ to higher energy. This Pt-Pt bond weakening has been referred to as "d-orbital frustration" previously. ${ }^{30}$

The $\Delta \mu$ signatures at various potentials for $\mathrm{Cl}^{-}, \mathrm{Br}^{-}$and $\mathrm{I}^{-}$at $10 \mathrm{mM}$ concentrations of $\mathrm{KX}$ (along with $1 \mathrm{M} \mathrm{HClO}_{4}$ ) are shown as an example of the experimental data in Figure $3.5 \mathrm{~b}, \mathrm{c}$ and d respectively. Note that compared to the "clean" electrolyte (no KX and only $1 \mathrm{M} \mathrm{HClO}_{4}$ ), a negative peak near the adsorption edge now appears for all three anions and at all potentials (except for the I- case above $1 \mathrm{~V}$ ). This negative feature now comes in part from halide coadsorption. Note that the negative peak amplitude decreases as the potential is increased, again because of initial $\mathrm{H}$ adsorption, then $\mathrm{OH}$, and finally $\mathrm{O}$, as occurs with the clean. As we previously reported, ${ }^{13}$ the larger negative feature near the absorption edge energy arises from halide adsorption. Further discussion is presented below. 


\subsubsection{FEFF 8.0 Results}

Figure 3.3 compares the FEFF8 calculated theoretical $\Delta \mu$ signatures for $\mathrm{Br}^{-}$and $\mathrm{I}^{-}$ with those for $\mathrm{Cl}$. Those for $\mathrm{Cl}^{-}$adsorption were previously reported, but are given here for comparison. For each halide adsorbate, the theoretical $\Delta \mu$ signatures for four different adsorption sites (a-top, bridge bonded, 3-fold face centered cubic and hexagonal closepacked) were calculated on a 6 atom Pt Janin cluster following eq. 2. The atop signature for $\mathrm{Cl}^{-}$exhibits a positive peak at around $0 \mathrm{eV}$ and then drops into a negative dip for the rest of the energy region, whereas the bridged bonded configuration shows a negative dip before a small positive peak at around $1 \mathrm{eV}$. The 3-fold face centered cubic (fcc) configuration shows a sharp negative peak at $-2 \mathrm{eV}$ followed by a broad positive peak in the positive energy region. The theoretical signatures for $\mathrm{Cl}^{-}$are different from that of $\mathrm{Br}^{-}$and $\mathrm{I}^{-}$in one major way. As illustrated in Figure 3 for $\mathrm{Br}^{-}$and $\mathrm{I}^{-}$, the four theoretical signatures for the atop, bridged ,fcc and hep configurations are very similar: they all show a negative peak at around $-2 \mathrm{eV}$ followed by a positive peak at around $4 \mathrm{eV}$. Thus for $\mathrm{Br}-$ and I- even the atop signature has the negative feature around $0 \mathrm{eV}$. Otherwise the $\Delta \mu$ signatures for all three halides mainly differ only in their relative intensities rather than their shape, and in general the magnitude of the negative feature near $0 \mathrm{eV}$ increases as the coordination number increases.

\subsubsection{Halide Adsorption and Rearrangement}

As illustrated previously, ${ }^{27,31}$ the $\Delta \mu$ peak amplitudes give an estimate of the relative anion adsorption on the surface, although the absolute monolayer coverage values are much harder to obtain. Figure 3.6 shows a plot of the absolute value of the negative $\Delta \mu$ 


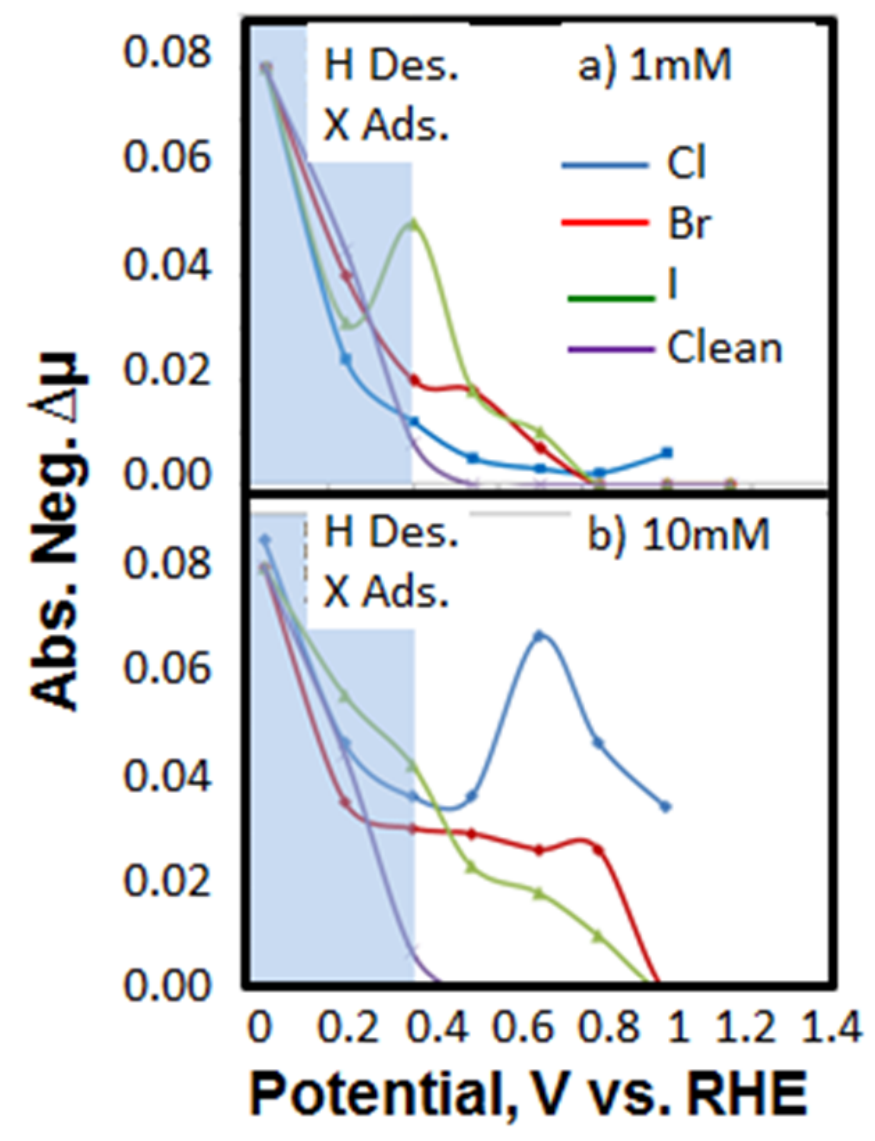

Figure 3.6 Plot of $\Delta \mu$ amplitudes for all three halides at a) $1 \mathrm{mM}$ and b) $10 \mathrm{mM}$ concentrations versus potential (vs. RHE). $\Delta \mu$ amplitudes for clean electrolyte $\left(1 \mathrm{M} \mathrm{HClO}_{4}\right)$ is also given in both cases for comparison purposes. All data has been normalized to 0.08 at $0.05 \mathrm{~V}$, as discussed in text. 
peak near $0 \mathrm{eV}$ for all three halide anions at both $1 \mathrm{mM}$ and $10 \mathrm{mM}$ concentrations as a function of potential. We use a procedure here practiced many times previously ${ }^{13,37.38}$. Since we are trying to distinguish between $\mathrm{O}(\mathrm{H})$ and $\mathrm{X}^{-}$, we plot the absolute value of the negative feature near $0 \mathrm{eV}$ in the experimental $\Delta \mu$ to emphasize the $\mathrm{X}$-adsorption, because the $\Delta \mu$ lineshape for $\mathrm{X}^{-}$has this feature and for $\mathrm{O}(\mathrm{H})$ it is small or even zero. In a latter section we will use the positive amplitude near $5 \mathrm{eV}$, to emphasize the $\mathrm{O}(\mathrm{H})$ adsorption.

The shaded area below $0.4 \mathrm{~V}$ is the potential where both hydrogen desorption and halide adsorption occur on the surface of Pt. As the $\Delta \mu$ line shapes for both n-fold H and Xhave the negative feature near $0 \mathrm{eV}, \mathrm{H}$ and $\mathrm{X}$ on the surface cannot be easily distinguished. Therefore, we focus instead on the region above $0.4 \mathrm{~V}$, and specifically the competition between halide adsorption and Pt-O formation, as this is the primary interest with regard to the ORR in a fuel cell anyway. We normalize all the of the $\Delta \mu$ amplitudes at $0.05 \mathrm{~V}$, since at this low potential all of the Pt surfaces should be relatively free of halide with only $\mathrm{H}_{\text {upd }}$ adsorbed; the $\mathrm{X}^{-}$having been driven off by the $\mathrm{H}$ adsorption. With this procedure, we are effectively scaling to constant electrochemical Pt surface area, such as is often done in an electrochemical environment when one determines the electrochemically active surface area (EASA) by estimating the $\mathrm{H}_{\text {upd }}$ current contribution from the $\mathrm{CV}$. We are scaling to constant ESA using the strong $\Delta \mu$ negative amplitude coming from the $\mathrm{H}$ adsorption at $0.05 \mathrm{~V}$. Although the halide adsorption strongly alters the $\mathrm{CV}$ in the $\mathrm{H}$ region (0.1 to 0.3 $\mathrm{V})$, at $0.05 \mathrm{~V}$, the halide is mostly driven off, so we can still use this $\Delta \mu$ normalization procedure. 
Note that the $\Delta \mu$ amplitude for the clean case drops monotonically to zero with increasing potential, due to desorption of $\mathrm{H}$, and that it is negligible above $0.4 \mathrm{~V}$ consistent with most of the $\mathrm{H}$ having desorbed at this point. Above $0.7 \mathrm{~V}, \mathrm{O}$ begins to adsorb, but since the negative $\Delta \mu$ amplitude near $0 \mathrm{eV}$ is negligible for $\mathrm{O}(\mathrm{H})$, the experimental $\Delta \mu$ amplitude remains small all the way up to $1 \mathrm{~V}$. Fig 3.6 shows a significant difference between the halide adsorption trends at lower concentration $(1 \mathrm{mM})$ vs. higher concentration $(10 \mathrm{mM})$ of $\mathrm{KX}$, so these will be discussed separately below.

\subsubsection{High $\mathrm{X}^{-}$Concentration Results}

Fig. $6 \mathrm{~b}$ shows that the negative $\Delta \mu$ amplitude for $10 \mathrm{mM} \mathrm{Cl}^{-}$initially decreases with potential below $0.5 \mathrm{~V}$, even though as shown previously ${ }^{13,16}$ the $\mathrm{Cl}^{-}$coverage increases strongly with potential between 0.2 and $0.6 \mathrm{~V}$ (i.e. as soon as the $\mathrm{H}$ is significantly off the surface). This initial decrease in the $\Delta \mu$ amplitude correlates with the formation of an "incommensurate" compressed halide adlayer, previously discussed by Lucas et. al ${ }^{16}$ and confirmed in our previous paper using the $\Delta \mu$-XANES and EXAFS analysis reported therein 13. Chloride does not form a closed packed mono-layer initially, but rather as the $\mathrm{Cl}^{-}$coverage increases the $\mathrm{Cl}$ anions are compressed together due to adatom-adatom interactions. This compression forces some $\mathrm{Cl}^{-}$anions into atop sites moving from hcp/fcc sites. The FEFF 8.0 calculations shown in Figure 3.3a indicate that $\mathrm{Cl}^{-}$adsorption in the atop configuration does not show the same large negative peak near $0 \mathrm{eV}$ as observed for $\mathrm{n}$ fold adsorption. Therefore the compression decreases the negative $\Delta \mu$ amplitude making the $\Delta \mu$ magnitude decrease with potential even though the coverage of $\mathrm{Cl}$ is increasing in 
this region. In our previous paper, ${ }^{13}$ the formation of the compression adlayer of $\mathrm{Cl}^{-}$was further confirmed by following the Pt-Pt coordination number $\left(\mathrm{N}_{\mathrm{Pt}-\mathrm{Pt}}\right)$ from EXAFS analysis. The $\mathrm{N}_{\mathrm{Pt}-\mathrm{Pt}}$ for $\mathrm{Cl}^{-}$increases between 0.3 and $0.5 \mathrm{~V}$ but decreases again between 0.5 and $0.7 \mathrm{~V}$. It has been shown previously that Pt-Pt coordination numbers increase when atop adsorption occurs, but decreases for $\mathrm{n}$-fold adsorption (observed for both halide addition and $\mathrm{O}, \mathrm{O}[\mathrm{H}]$ adsorption]. This perfectly correlates with displacement of some $\mathrm{n}$ fold anions to atop positions with compression of the halide adlayer as the potential increases below $0.5 \mathrm{~V}$. Above $0.6 \mathrm{~V}$, the $\mathrm{Cl}$ layer has sufficiently compressed and opened up clean $\mathrm{n}$-fold sites to allow further $\mathrm{n}$-fold $\mathrm{Cl}$ adsorption, thus increasing the negative $\Delta \mu$ amplitude and decreasing $\mathrm{N}_{\mathrm{Pt}-\mathrm{Pt}}$. Above $0.75 \mathrm{~V}, \mathrm{O}(\mathrm{H})$ adsorption forces $\mathrm{Cl}$ to desorb, thus decreasing $\Delta \mu$ again. All of this behavior for $\mathrm{Cl}$ has been discussed in our previous paper.

As indicated by Lucas et. al ${ }^{16}$ utilizing in-situ surface X-ray scattering (SXS) studies, the structure of the halide adlayer formed on a $\mathrm{Pt}(111)$ surface is directly related to the interaction strength between the adatom and the adsorbing surface: higher adatom-adatom interaction leads to formation of an "incommensurate" or disordered adlayer structure relative to the surface, whereas a higher adatom-surface interaction leads to an ordered commensurate adlayer. Thus, as the Pt-X bond strength increases in the order $\mathrm{Cl}^{-}<\mathrm{Br}^{--}<\mathrm{I}^{-}$ , we expect to see a more structured adlayer formed on the surface of Pt. Further the increasing size of the halide allows less compression. Finally the difference between the atop and $\mathrm{n}$-fold sites is much smaller for $\mathrm{Br}$ and I than for $\mathrm{Cl}$. The $\Delta \mu$ results in Fig. $6 \mathrm{~b}$ confirm these trends as the incommensurate compression and then additional adsorption evident in the $\mathrm{Cl}^{-}$case becomes increasingly less evident (if it forms at all) in the "double- 
layer" region for $\mathrm{Br}^{-}$and $\mathrm{I}^{-}$. Indeed, the SXS studies of Lucas confirm a much more ordered adlayer and relatively constant coverage between 0.5 and $0.8 \mathrm{~V}$ for $\mathrm{I}^{-}$and $\mathrm{Br}^{-}$consistent with Fig. 6b.

\subsubsection{Low Concentration Results}

The $\Delta \mu$ amplitude plot for halide adsorption at $1 \mathrm{mM} \mathrm{KX}$ concentration, as illustrated in Figure 3.6a, follows a completely different trend from that of the $10 \mathrm{mM}$ concentration. Not only are the relative magnitudes different, at $1 \mathrm{mM}$ all of the $\Delta \mu$ amplitudes fall nearly monotonically beginning already at 0 . V. This occurs despite evidence in the literature that the X- coverage on the surface is relatively flat in the region between 0.4 and $0.7 \mathrm{~V}$. Clearly again the $\Delta \mu$ amplitude is not following the relative $\mathrm{X}^{-}$coverage with potential.

How do we explain this? The drop off in $\Delta \mu$ magnitude can be explained based on the changing nature of the interaction with the $\operatorname{Pt}(111)$ surface with increased electrode potential. As the potential increases, the charge and hence the electronegativity of the Pt surface atoms increases. This increased positive charge density on the surface Pt atoms will have an effect on the nature of the Pt-halide bond, particularly on the highly polarizable $\mathrm{X}^{-}$ anions and the relatively weak $\mathrm{Pt}-\mathrm{X}^{-}$interaction; changing it from a more ionic to a more covalent one. By relatively weak here, we are referring to the much stronger and covalent Pt-H or Pt-O bonds; indeed we would not expect much change between these latter bonds with potential, rather the $\mathrm{O}(\mathrm{H})$ coverage just increases with potential. The increased covalency of the Pt-X bond we believe can account for the decrease in $\Delta \mu$ amplitudes. 
To confirm this hypothesis, additional FEFF 8.0 calculations were conducted. Figure 3.7 compares the 4 -fold hep set of theoretical $\Delta \mu$ signatures for $\mathrm{Cl}^{-}, \mathrm{Br}^{-}$and $\mathrm{I}^{-}$with one performed to simulate the effect of increased potential on the electrode. To simulate this, an electronegative fluoride atom was added in an atop position to the Pt atom at the bottom of the $\mathrm{Pt}_{6}$ cluster. Adding a highly electronegative atom such as fluoride draws electron density away from the upper photon adsorbing atom and increases the positive charge density on these Pt atoms. This basically has the same effect as increased electrode potential. Thus, the two $\Delta \mu$ presented in Figure 7 are:

1. $\Delta \mu$ (no fluoride) $=\mu\left(\mathrm{X} / \mathrm{Pt}_{6}\right)-\mu\left(\mathrm{Pt}_{6}\right)$

2. $\Delta \mu$ (with fluoride) $=\mu\left(\mathrm{X} / \mathrm{Pt}_{6}-\mathrm{F}\right)-\mu\left(\mathrm{Pt}_{6}-\mathrm{F}\right)$

where $\mathrm{X}=\mathrm{Cl}^{-}, \mathrm{Br}^{-}$and $\mathrm{I}^{-}$.

In all of these cases, the halide anion is placed in the 4-fold hcp site. As can be observed in Fig. 3.7, the addition of fluoride in all three cases decreases the $\Delta \mu$ negataive amplitude for all halide anions by about 0.02 . These results confirm that the effect of increased Pt positive charge density and covalency of the bond decreases the $\Delta \mu$ negative amplitudes. This decrease is somewhat surprisingly almost equal for all three anions. One might have expected that as the anion increased in size, its increased polarizability would have caused this effect to become larger, but that is not the case. Thus the general drop in $\Delta \mu$ intensity at $1 \mathrm{mM} \mathrm{KX}$ concentration is due to the increasing covalency of the Pt-X bond, and that indeed the coverages of $\mathrm{X}$ on the surface at this concentration is relatively constant. Further this effect is similar for all three halides. 


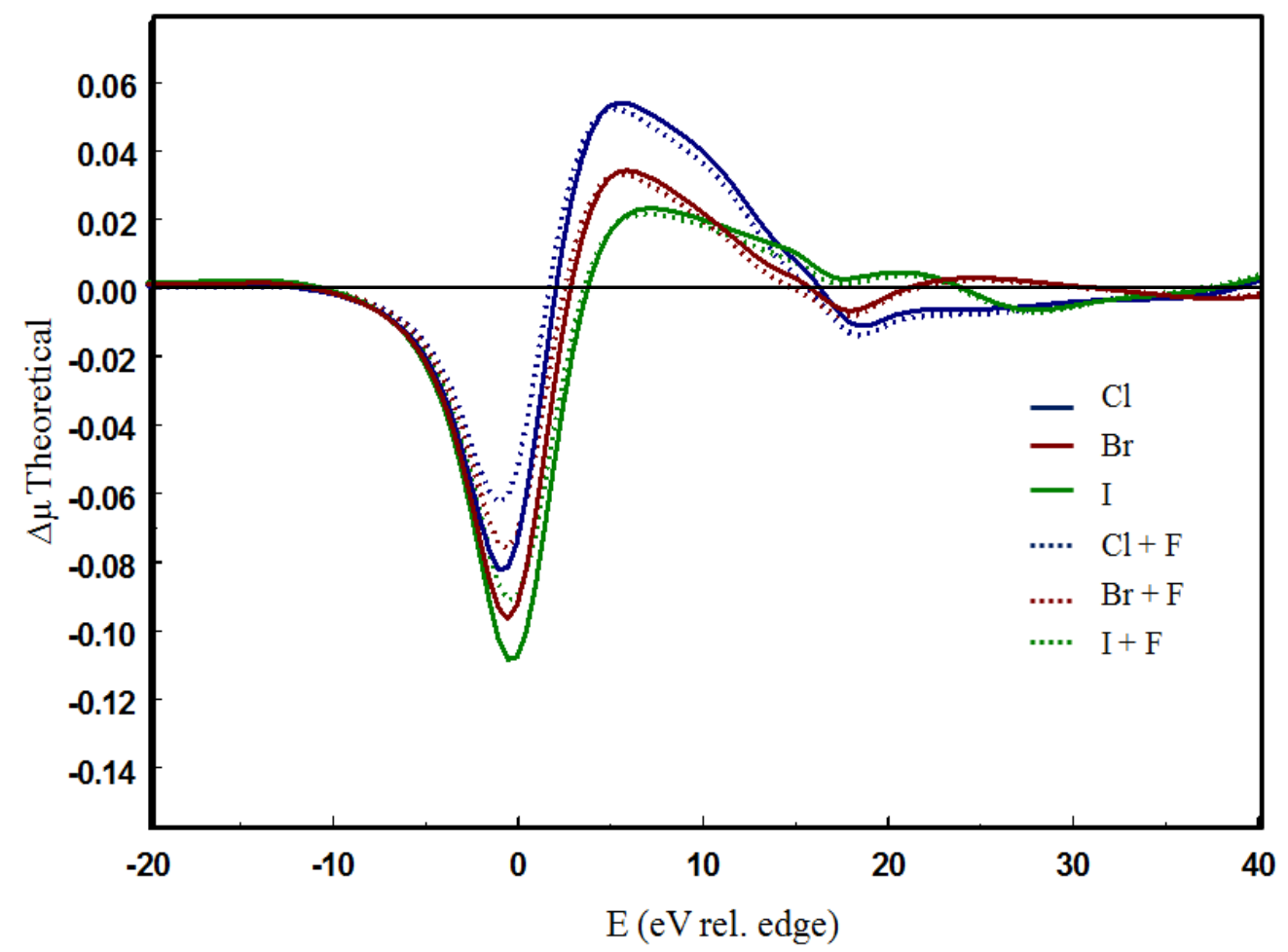

Figure 3.7 Theoretical $\Delta \mu_{\mathrm{t}}=\mu\left(\mathrm{Pt}_{6}-\mathrm{X}+\mathrm{F}\right)-\mu\left(\mathrm{Pt}_{6}\right)$ signatures; where $\mathrm{X}$ is $\mathrm{Cl}^{-}, \mathrm{Br}^{-}$and $\mathrm{I}^{-}$in a),b) and c) respectively. In all three cases $\mathrm{F}$ atom is placed in atop position on neighboring $\mathrm{Pt}$ atom with respect to adsorbing $\mathrm{Pt}$ atom. The $\mathrm{Pt}-\mathrm{X}$ bond distances are calculated based on atomic radii, and are: $2.06 \mathrm{~A}^{\circ}, 2.35 \mathrm{~A}^{\circ}, 2.50 \mathrm{~A}^{\circ}$ and $2.75 \mathrm{~A}^{\circ}$ for Pt-F, Pt-Cl, Pt-Br and Pt-I respectively. 
We should also comment on the relative magnitudes of the $\Delta \mu$ at $1 \mathrm{mM}$ vs. $10 \mathrm{mM}$ $\mathrm{KX}$. At $1 \mathrm{mM} \mathrm{KX}$, the magnitudes generally go as $\mathrm{I}>\mathrm{Br}>\mathrm{Cl}$, and at $10 \mathrm{mM}$ the reverse is true. Clearly at low KX concentration, the coverage is determined by the relative bond strength, and therefore the trend at $1 \mathrm{mM}$ follows the known Pt-X bond strength trend; Pt-I $>\mathrm{Pt}-\mathrm{Br}>\mathrm{Pt}-\mathrm{Cl}$. This is consistent with earlier studies ${ }^{16}$ and our electrochemical results: I is adsorbed more strongly on the surface compared to $\mathrm{Br}$ and $\mathrm{Cl}$, and, as shown in the $\mathrm{CV}$ results in Figure 2b, $\mathrm{I}^{-}$has a higher poisoning effect than $\mathrm{Br}^{-}$even at lower concentrations. At $10 \mathrm{mM} \mathrm{KX}$, generally full coverage is achieved, and the smaller $\mathrm{Cl}^{-}$anion radius and compression as discussed above allows greater coverage and hence enhanced $\mathrm{Pt}-\mathrm{Cl}$ coordination. These trends mean that $\Delta \mu$ is in general following the relative $\mathrm{X}$ coverage (i.e., $\mathrm{Cl}$ relative to $\mathrm{Br}$ relative to $\mathrm{I}$ ) on the surface at each potential, but as the potential increases and the electronic charge on the surface Pt atoms increase, the $\Delta \mu$ falls off, even though the coverage is staying relative constant.

We note that previously we have assumed that $\Delta \mu$ was a good indicator of adsorbate coverage with potential, but that was for more covalent Pt-adsorbate bonds such as with $\mathrm{H}$ and $\mathrm{O}$ as noted above ${ }^{13,27,31}$. Further we believe that this changing covalent character is much more important at lower X coverage, and hence the $\Delta \mu$ results at $10 \mathrm{mM}$ probably are reflecting more closely the relative coverage of $X$ with potential than those at $1 \mathrm{mM}$, but the effect is clearly present at some level at both concentrations. 


\subsubsection{Water Activation on Pt(111) in Presence of Bromide and Iodide}

Figure 3.8 shows another set of $\Delta \mu$ plots for potentials between 0.7 and $1.00 \mathrm{~V}$. Here, the reference spectrum used is the spectrum collected at $0.54 \mathrm{~V}$ in the same electrolyte as the other scans (containing the halides), therefore $\Delta \mu$ is now determined as follows:

$\Delta \mu=\mu(\mathrm{xV}, 10 \mathrm{mM}$ halide $)-\mu(0.54 \mathrm{~V}, 10 \mathrm{mM}$ halide $)$

This is in contrast to the data above where the reference was the $\mu$ without any halide, so that the $\Delta \mu$ difference emphasized the effect of $\mathrm{X}$ adsorption. Now we want to follow the coverage of $\mathrm{O}$ with potential and determine the effect of halide adsorption on the $\mathrm{O}$ coverage. Since the onset of oxygen adsorption is expected to occur above $0.7 \mathrm{~V}$, the $\Delta \mu$ constructed following Eq. 8 should reveal the $\mathrm{Pt}-\mathrm{O}[\mathrm{H}]$ signature. Comparison of these results with the FEFF8.0 theoretical $\mathrm{O}[\mathrm{H}]$ and $\mathrm{O}$ adsorption $\Delta \mu$ line shapes illustrated in Figure 3.4 in fact confirms this, and shows the onset of $\mathrm{OH}$ (atop) adsorption on $\mathrm{Pt}$ in both the $10 \mathrm{mM} \mathrm{Br}^{-}$and $\mathrm{I}^{-}$electrolytes. Both $\mathrm{Br}^{-}$and $\mathrm{I}^{-}$exhibit a positive peak around $2 \mathrm{eV}$, for both electrolytes, as shown in Figure 8c, shows the poisoning effects of these anions. As

expected, $\mathrm{Br}^{-}$has larger $\Delta \mu$ amplitudes than $\mathrm{I}^{-}$, which means that $\mathrm{I}^{-}$suppresses the adsorption of oxygen more than $\mathrm{Br}^{-}$(and $\mathrm{Cl}^{-}$as compared to previously reported results).

That I shows a higher poisoning effect than $\mathrm{Br}$, means it is forced off the surface with more difficultly than the $\mathrm{Br}$, and this correlates with their relative Pt interaction strengths. 


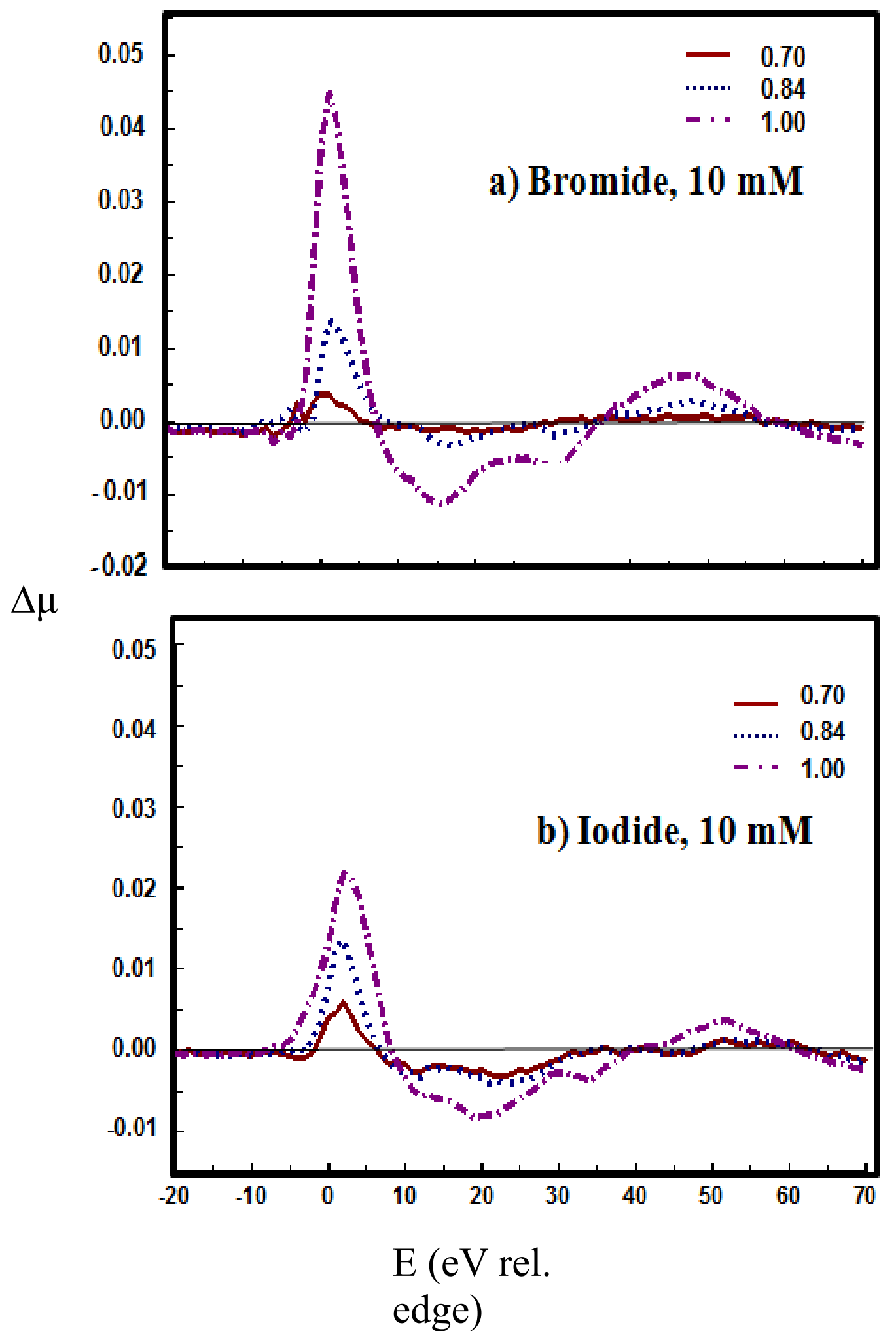




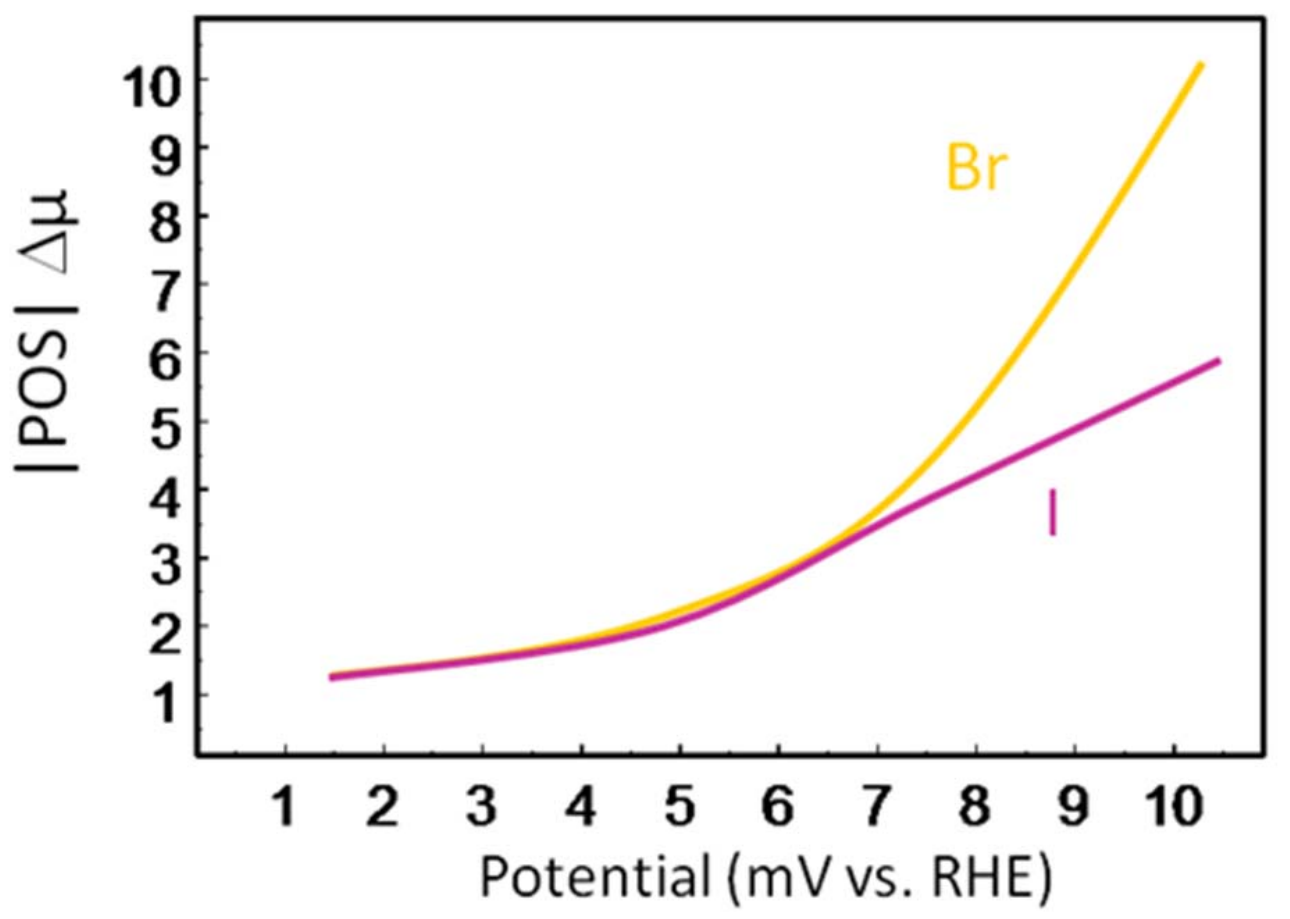

Figure 3.8a and b. Pt $\mathrm{L}_{3}$ edge $\Delta \mu=\mu(\mathrm{V}$, halide $)-\mu(0.54 \mathrm{~V}$ clean $)$ spectra for $30 \mathrm{wt} \% \mathrm{Pt} / \mathrm{C}$ in $1 \mathrm{M} \mathrm{HClO} 4$ and $10 \mathrm{mM}$ of a) bromide and b)iodide, clean spectrum in this case is XAS spectrum collected at $0.54 \mathrm{~V}$ in the same electrolyte as spectra collected at other potentials, hence reference spectrum also contains $10 \mathrm{mM}$ of bromide and iodide in a and $\mathrm{b}$ respectively. Figure $8 \mathrm{c}$ shows $\Delta \mu$ amplitudes vs. potential correlating with parts b and $\mathrm{c}$. 


\subsection{Summary and Conclusions:}

A combination of electrochemical and in-situ XAS studies on bromide and iodide adsorption on polycrystalline supported $\mathrm{Pt} / \mathrm{C}$ reveals the relative poisoning effects of these halide anions on reducing the ORR activity. In the case of $\mathrm{Br}-$, even concentrations as low as $10^{-3} \mathrm{M}$ caused an overpotential increase of ca. $200 \mathrm{mV}$, and the overpotential increased to even higher levels $(150 \mathrm{mV})$ when the concentration is increased to $10^{-2} \mathrm{M}$. The CVs in the presence of $\mathrm{Br}^{-}$were also affected both in the $\mathrm{H}_{\text {upd }}$ and double-layer regions. At concentration above $10^{-3} \mathrm{M}$, Pt-O formation is greatly affected by the presence of bromide. At concentrations above $10^{-2}$ a large anodic current is observed due to $\mathrm{Br}_{2}$ evolution at 1.0 V.

In the presence of iodide, the CVs were even more affected. Even at a concentration as low as $10^{-5} \mathrm{M}$ all three regions of the $\mathrm{CV}$ were greatly affected, with $\mathrm{H}_{\text {upd }}$ losing all features and Pt-O completely suppressed up to high potentials. All these effects were enhanced with increasing concentration; at $10^{-3} \mathrm{M}$ a redox couple appeared which was identified as the $\mathrm{I}_{2} / \mathrm{I}_{3}$ couple.

The increased poisoning effect of bromide and iodide relative to chloride was also observed in the $\Delta \mu$ results. The in-situ XAS studies were conducted on two KX concentrations: $1 \mathrm{mM}$ and $10 \mathrm{mM}$. The stronger poisoning effects were observed for both $\mathrm{Br}^{-}$and $\mathrm{I}^{-}$compared to that of $\mathrm{Cl}^{-}$in $1 \mathrm{mM}$ concentration, where the higher halide coverages (associated with the $\Delta \mu$ negative magnitudes) were observed for both $\mathrm{I}^{-}$and $\mathrm{Br}-$. All halide coverages decreased with increase of potential above $1.0 \mathrm{~V}$ due to oxygen adsorption 
which forces the halides off of the surface. It is not possible to distinguish the $\mathrm{Br}$ and $\mathrm{I}$ adsorption sites, as all four different adsorption sites (atop, bridged, fcc and hcp) for both bromide and iodide have somewhat similar theoretical signatures with the only difference being the increase of the negative $\Delta \mu$ amplitudes with increasing n-fold coordination. At higher halide concentration $(10 \mathrm{mM})$, however, chloride had higher $\Delta \mu$ amplitudes than that of bromide and iodide suggesting that at this concentration the halide coverages are nearly full, and the increased $\Delta \mu$ is reflecting the greater Pt-X coordination of the smaller compressed $\mathrm{Cl}$ adlayer, with a similar but much smaller effect for the $\mathrm{Br}$ and I adlayers.

Finally, we conclude that the $\Delta \mu$ magnitude is affected by the varying nature of the Pthalide bond, at least at low coverage. As the potential increases, the Pt-halide bond becomes more covalent and this results in the decrease of $\Delta \mu$. The changing nature of the Pt-halide bond was confirmed by simulating the potential effect on Pt through addition of a highly electronegative $\mathrm{F}$ atom to the $\mathrm{Pt}_{6}$ cluster. This illustrated that for all three anions an increased positive charge density on the adsorbing atom decreases the negative $\Delta \mu$ amplitude of the peak near $0 \mathrm{eV}$. This effect is observed equally for all of the halides despite the changing anion size, electronegativity, and polorizability, so that the halide coverage relative to each other is consistently tracking the expected actual coverage, even though the relative coverage with potential for the halides is not consistently reflecting the true coverage. It has been assumed over the years that the magnitude of $\Delta \mu$ consistently tracks the relative adsorbate coverage with potential, and this is still believed to be the case for such strongly bonded adsorbates as $\mathrm{OH}$ and $\mathrm{O}$; however for the weak and highly 
polarizable halide anions, this apparently may not be the case, particularly at low coverages.

Finally, experimental $\Delta \mu$ results with the reference $\mu$ collected at $0.54 \mathrm{~V}$ in the halide containing solution showed that the onset of oxygen adsorption around $0.7 \mathrm{~V}$, moves up in the order $\mathrm{I}>\mathrm{Br}>\mathrm{Cl}$. This correlates with the electrochemical results and the relative Pt-X adsorption strengths of the halides.

\section{Acknowledgments:}

Financial support for this project was provided by the Army Research Office via both a single investigator grant and a Multi University Research Initiative (Case Western Research University, P.I.). The authors are grateful for the use of X11-B at the National Synchrotron Light Source, Brookhaven National Laboratory, Upton, NY, which is supported by the U.S. Department of Energy, Office of Science, Office of Basic Energy Sciences, under Contract No. DE-AC02-98CH10886. 


\subsection{References:}

(1) Wang, J. X.; Markovic, N. M.; Adzic, R. R. Kinetic Analysis of Oxygen Reduction on $\operatorname{Pt}(111)$ in Acid Solutions: Intrinsic KineticParameters and Anion Adsorption Effects. J. Phys. Chem. B 2004, 108, 4127-4133.

(2) El Kadiri, F.; Faure, R.; Durand, R. Electrochemical reduction of molecular oxygen on platinumsingle crystals. J. Electroanal. Chem. 1991, 301, 177-188.

(3) Damjanovic, A.; Genshaw, M. A.; Bockris, J. The Role of Hydrogen Peroxide in Oxygen Reduction at Platinum in $\mathrm{H}_{2} \mathrm{SO}_{4}$ Solution. J. Electrochem. Soc. 1967, 114, 466472 .

(4) Adzic, R. R.; Tripkovic, A. V.; Markovic, N. M. Oxygen Reduction on Electored Surfaces Modified by Foreign Metal Ad-atoms: Lead Ad-atoms on Gold. J. Electroanal. Chem. 1980, 114, 37-51.

(5) Watanabe, M.; Uchida, M.; Motoo, S. Preparation of highly dispersed Pt $+\mathrm{Ru}$ alloy clusters and the activity for the electrooxidation of methanol.J. Electroanal. Chem. 1987, 229, 395-406.

(6) H. G. Petrow, H. G.; Allen, R. J. USA Patent 3992 331, 1976.

(7) Luczak, F. J.; Landsman, D. A. USA Patent 4447 506, 1984.

(8) Paulus, U. A.; Schmidt, T. J.; Gasteiger, H. A. In Poisons for the O2 reduction reaction; Vielstich, W., Gasteiger, H. A., Lamm, A. and Yokokawa, H., Eds.; Handbook of Fuel Cells - Fundamentals, Technology and Applications; John Wiley \& Sons, Ltd.: 2010;

(9) Zhang, L.; Sanjeev Mukerjee, S. Investigation of Durability Issues of Selected Nonflourinated Proton Exchange Membranes for Fuel Cell Application. J. Electrochem. Soc. 2006, 153, A1062.

(10) Breiter, M. W. Voltammetric Study of Halide Ion Adsorption on Platinum in Perchloric Acid Solutions. Electrochim. Acta 1963, 8, 925.

(11) Huang, J. C.; O'Grady, W. E.; Yeager, E. The Effects of Cations and Anions on Hydrogen Chemisorption at Pt. J. Electrochem. Soc. 1977, 124, 1732.

(12) Horanyi, G.; Rizmayer, E. M. The Problem of the Irreversibility of the Adsorption of Halides on Platinum. J. Electroanal. Chem. 1977, 83, 367. 
(13) Arruda, T. M.; Shyam, B.; Ziegelbauer, J. M.; Mukerjee, S.; Ramaker, D. E. Investigation into the Competitive and Site-Specific Nature of Anion Adsorption on PtUsing In Situ X-ray Absorption Spectroscopy. J. Phys. Chem. C 2008, 112, 18087.

(14) Markovic, N.; Ross, P. N. The effect of specific adsorption of ions and underpotential deposition of copper on the electro-oxidation of methanol on platinum single-crystal surfaces.J. Electroanal. Chem. 1992, 330, 499.

(15) Campbell, D. J.; Lynch, M. L.; Corn, R. M. Second harmonic generation studies of anionic chemisorption at polycrystalline platinum electrodes. Langmuir 1990, 6, 1656.

(16) Lucas, C. A.; Markovic, N. M.; Ross, P. N. Adsorption of halide anions at the $\mathrm{Pt}(111)$-solution interfacestudied by in situ surface x-ray scattering. Phys. Rev. B 1997, $55,7964$.

(17) Markovic', N. M.; Ross, P. N. Surface Science Studies of Model Fuel Cell Electrocatalysts. Surf. Sci. Rep. 2002, 45, 117.

(18) McBreen, J.; O’Grady, W. E.; Pandya, K. I.; Hoffman, R. W.; Sayers, D. E. EXAFS study of the nickel oxide electrode. Langmuir 1987, 3, 428.

(19) Newville, M. J. IFEFFIT: interactive XAFS analysis and FEFF fitting. $J$. Synchrotron Radiation 2001, 8, 322.

(20) Newville, M.; Livins, P.; Yacoby, Y.; Stern, E. A.; Rehr, J. J. Near-edge X-rayabsorption fine structure of $\mathrm{Pb}$ : A comparison of theory and experiment. Phys. Rev. $B$ 1993, 47, 14126.

(21) Koningsberger, D. C.; Mojet, B. L.; Miller, J. T.; Ramaker, D. E. XAFS spectroscopy in catalysis research: AXAFS and shape resonances. J. Synchrotron Radiation 1999, 6, 135.

(22) Ramaker, D. E.; Mojet, B. L.; Koningsberger, D. C.; O’Grady, W. E. Understanding atomic x-ray absorption fine structure in x-ray absorption spectra. $J$. Phys.: Condens. Matter. 1998, 10, 8753.

(23) Ramaker, D. E.; Mojet, B. L.; Miller, J. T.; Koningsberger, D. C. An atomic Xray absorption fine structure study of the influence of hydrogen chemisorption and support on the electronic structure of supported Pt particles . Top. Catal. 2000, 10, 157.

(24) van Dorssen, G. E.; Koningsberger, D. C.; Ramaker, D. E. Separation of double-electron and atomic XAFS contributions in X-ray absorption spectra of Pt foil and $\mathrm{Na}_{2} \mathrm{Pt}(\mathrm{OH})_{6}$. J. Phys.: Condens. Matter. 2002, 14, 13529. 
(25) Janin, E.; von Schenck, H.; Go“thelid, M.; Karlsson, U. O. Bridge-bonded atomic oxygen on $\mathrm{Pt}(110)$. Phys. Rev. B 2000, 61, 13144.

(26) Marković, N. M.; Gasteiger, H. A.; Grgur, B. N.; Ross, P. N., J. Oxygen reduction reaction on $\mathrm{Pt}(111)$ : effects of bromide . J. Electroanal. Chem. 1999, 467, 157.

(27) Teliska, M.; O’Grady, W. E.; Ramaker, D. E. Determination of O and OH Adsorption Sites and Coverage in Situ on Pt Electrodes from $\mathrm{Pt} \mathrm{L}_{23} \mathrm{X}$-ray Absorption Spectroscopy. J. Phys. Chem. B 2005, 109, 8076.

(28) Balbuena, P. B.; Altomare, D.; Vadlamani, N.; Bingi, S.; Agapito, L. A.; Seminario, J. M. Adsorption of $\mathrm{O}, \mathrm{OH}$, and $\mathrm{H}_{2} \mathrm{O}$ on Pt-Based Bimetallic Clusters Alloyed with Co, Cr, and Ni.J. Phys. Chem. A 2004, 108, 6378.

(29) Xu, Y.; Ruban, A. V.; Mavrikakis, M. Adsorption and Dissociation of $\mathrm{O}_{2}$ on Pt-Co and Pt-Fe Alloys. J. Am. Chem. Soc. 2004, 126, 4717.

(30) Fiebelman, P. J. $d$-electron frustration and the large fcc versus hcp binding preference in $\mathrm{O}$ adsorption on $\mathrm{Pt}(111)$. J. Phys. Rev. B 1997, 56, 10532.

(31) Teliska, M.; Murthi, V. S.; Mukerjee, S.; Ramaker, D. E. Correlation of Water Activation, Surface Properties, and Oxygen Reduction Reactivity of Supported Pt-M/C Bimetallic Electrocatalysts Using XAS. J. Electrochem. Soc. 2005, 152, A2159.

(32) Jerkiewicz, G.; Vatankhah, G.; Lessard, J.; Soriaga, M. P.; Park, Y. -. Surfaceoxide growth at platinum electrodes in aqueous $\mathrm{H}_{2} \mathrm{SO}_{4}$ : Reexamination of its mechanism through combined cyclic-voltammetry, electrochemical quartz-crystal nanobalance, and Auger electron spectroscopy measurements. Electrochim. Acta 2004, 49, 1451.

(33) Zolfaghari, A.; Conway, B. E.; Jerkiewicz, G. Elucidation of the effects of competitive adsorption of $\mathrm{Cl}^{-}$and $\mathrm{Br}^{-}$ions on the initial stages of $\mathrm{Pt}$ surface oxidation by means of electrochemical nanogravimetry. Electrochim. Acta 2002, 47, 1173.

(34) Ramaker, D. E.; Oudenhuijzen, M. K.; Koningsberger, D. C. J.Phys. Chem. B 2005, 109, 5608. J. Phys. Chem. B 2005, 109, 5608.

(35) Kinoshita, K. Particle Size Effects for Oxygen Reduction on Highly Dispersed Platinum in Acid Electrolytes. J. Electrochem. Soc. 1990, 137, 845.

(36) Teliska, M.; O’Grady, W.E.; Ramaker, D.E.; Determination of H Adsorption sites on Pt/C Electrodes in HClO4 from Pt L23 X-ray Absorption Spectroscopy, J. Phys. Chem. B 2004,108, 2333. 
(37) He, Q.; Shyam, B.; Nishijima,M.; Ramaker, D.; Mukerjee, S.; Mitigating Phosphate Anion Poisoning of Cathodic Pt/C Catalysis in Phosphoric Acid Fuel Cells, Manuscript submitted to J.Phys. Chem

(38) Teliska, M.; Murthis, V.S.; Mukerjee, S.; Rmakaer, D.E., "Site-Specific vs. Specific Adsorption of Anions on Pt and Pt Based Alloys"; J. Phys. Chem. C, 2007, 111, 9267. 


\section{Chapter 4: Space Resolved, In Operando X-ray Absorption Spectroscopy: Investigations on both the Anode and Cathode in a DMF}

\subsection{Introduction}

Direct methanol fuel cells (DMFC) are promising energy storage devices for future portable applications. Although DMFCs offer the advantages of high energy density, easy liquid fuel storage and refilling, and simple system structure, they also face technical challenges ${ }^{1}$. One of the main challenges in operating DMFCs is the non-uniformity in current distribution across a single cell. These current variations can arise from a variety of reasons, such as from variations in catalyst loading or feed supply across the cell, blocking or flooding of the membrane-electrode assembly (MEA) surface by gaseous or water products, and non-uniformities in the temperature distribution. These variations can be large; so large that the coexistence of local galvanic and electrolytic domains has been observed in a single channel DMFC ${ }^{2}$. At a local electrolytic domain, electrolysis occurs in contrast to the normal galvanic fuel cell process (e.g., methanol oxidation and oxygen reduction). Variations in current distribution generally lead to increased aging of the catalyst, a decrease in power output, and can cause local over-heating and other negative consequences for the operation of the $\mathrm{DMFC}^{3}$.

One of the main sources of uneven current distribution across a catalyst is the inhomogeneous gas (feed) supply over the cell on both the cathodic and anodic sides. For example a non-uniform distribution of methanol on the anode is highly detrimental as it can lead to cell reversal and can accelerate the degradation of the Pt catalyst ${ }^{4}$. Further, the evolution of $\mathrm{CO}_{2}$ bubbles can inhibit a homogenous supply of methanol feed, whereas on 
the cathodic side water droplets can flood the MEA surface and block the oxygen supply ${ }^{3}$. In extreme cases a non-uniform distribution of methanol on the anode can lead to cell reversal and can accelerate the degradation of the Pt catalyst ${ }^{4}$. Hence, knowledge of the spatial distribution of the adsorbates on the surface and their behavior under different operational modes can provide vital information to future fuel cell design.

Various techniques have been previously employed to study the correlation between current distribution and spatial distribution of adsorbates on the surface; namely neutron radiography ${ }^{5}$, X-ray radiography ${ }^{6}$, transmission electron microscopy (TEM) ${ }^{7}$, and current distribution studies using segmented sensor plates ${ }^{8}$, etc. While these techniques have provided valuable information on mass transport phenomena and it's correlation with the inhomogeneity in current distribution, the effects of adsorbate coverage on catalytic surface activity and performance have not been fully explored.

The current distribution in fuel cells has also been studied at the cathode with different $\mathrm{O}_{2}$ flow rates. Previous studies ${ }^{8-11}$ have shown that under insufficient oxygen supply, the cathode active area divides into different operational regimes. The area closest to the cathode inlet with higher oxygen concentration will operate in the normal DMFC mode (galvanic), whereas in the oxygen deprived areas near the outlet and under certain conditions, the cathode may start working in an electrolytic regime ${ }^{9,10}$. Under certain oxygen starvation conditions at the cathode, the methanol, which has permeated through the membrane from the anodic side (methanol cross-over), can interfere seriously with the normal oxygen reduction reaction (ORR). Instead of the ORR, in this area methanol is electrochemically oxidized to produce protons. The protons can then move through the 
membrane to the anode (reverse of the normal direction), to reduce to dihydrogen. It has also been shown that short operation of the fuel cell in low oxygen flow can enhance the galvanic performance of the cell ${ }^{11}$. Thus, studying the surface coverage under low air flow using XAS can also give insight into the activation of the surface under these conditions.

As summarized above, spatially resolved information over the area of a fuel cell electrode can be obtained by various imaging techniques using X-Ray, neutrons etc., or by current distribution measurements using segmented sensor plates. With X-Ray and neutron imaging techniques, one can directly visualize the mass transport and water evolution in a working fuel cell, thus giving insight into the fuel and water management ${ }^{5,6}$. In contrast, spatially resolved current distribution measurements can provide the current density at each segment in a fuel cell, which may be then correlated to various mass transport phenomena. For example, Park et al ${ }^{12}$ have reported extensive investigations on the influence of the current distribution on various operating parameters like the oxygen and methanol flow rate, output current, temperature and flow field geometry. More recently Schroeder et al ${ }^{5}$ combined both neutron imaging as well as current distribution measurements to correlate the GDL wettability on fuel cell performance. Although these techniques provide significant information about the mass transport and current distribution over the area of the catalyst, at the same time they are limited by the fact that they don't provide information regarding the state of the catalyst; e.g. adsorbates present, stability, and degradation.

One of the few methods that can provide adsorbate coverages in an operating fuel 
cell on real practical catalysts is x-ray absorption spectroscopy (XAS) using the $\Delta \mu-$ XANES analysis technique ${ }^{13-16}$. Usually XAS is used to investigate catalyst morphology and composition from the extended $\mathrm{x}$-ray absorption fine structure (EXAFS) region, but the $\mathrm{x}$-ray absorption near edge structure (XANES) region also contains information about binding site and oxidative state of the absorbing atom. The $\Delta \mu$-XANES method takes out the atomic "background" contribution to enhance the contribution due to adsorbates, and then by comparison with theoretical FEFF8 results provides both coverage and binding site information for adsorbates such as $\mathrm{CO}, \mathrm{OH}, \mathrm{O}$, and $\mathrm{H}^{13,16}$.

From the neutron imaging studies in bifunctional operation mentioned above ${ }^{9}$, it may be anticipated that there exists a "cross talk" across the PEM, or spatial correlation between the anode and cathode, depending on the reactant concentration, so that the electrode adsorbate coverages cannot be fully understood independently from each other. However, this "cross talk" or spatial correlation has so far only been observed indirectly by measuring the water and current distribution locally. In this work X-ray absorption spectroscopy (XAS) is utilized to study the spatial distribution of adsorbates on an MEA in both the anodic and cathodic compartments "in parallel" under various methanol and oxygen flow rates, so that we can observe this "cross-talk" and the effects it has on the adsorbate coverage directly. A special in-situ cell was designed to investigate the spatial distribution of adsorbates.

The $\mathrm{CO}$ and $\mathrm{OH}$ coverage on a $\mathrm{Pt} / \mathrm{Ru}$ anode surface in a DMFC as a function of applied potential have been studied previously utilizing the $\Delta \mu$-XANES and EXAFS analysis techniques ${ }^{15,16}$. The CO oxidation process, most often the rate determining step in 
methanol oxidation on an alloyed $\mathrm{Pt} / \mathrm{Ru}$ anode, can proceed via three different mechanisms, distinguished by the source of the $\mathrm{OH}$ involved in the $\mathrm{CO}$ oxidation: 1). The bifunctional mechanism (BF) which dominates at potentials below $0.25 \mathrm{~V}$ vs. RHE, this mechanism involves formation of $\mathrm{OH}$ from water activation on $\mathrm{Ru}$ islands, and subsequently oxidizing the CO;2) The direct ligand (DL) mechanism dominates at potentials between 0.25 to $0.4 \mathrm{~V}$ vs. RHE. This mechanism involves the activation of water and $\mathrm{OH}$ formation directly on the Pt surface but near the Ru islands. This water activation on Pt atoms is facilitated at lower potentials due to the ligand or electronic effect from the nearby Ru islands; and 3) The direct (D) mechanism, which occurs at potentials greater than $0.4 \mathrm{~V}$ vs. RHE and on the Pt atoms far from Ru islands ${ }^{15,16}$. The dominant $\mathrm{CO}$ oxidation mechanism on the anode thus depends on the potential region the fuel cell is operating at. The objective here is to investigate the $\mathrm{CO}$ oxidation mechanism at various segments or windows of the anode and at different potentials, and to correlate this with the oxidation reduction occurring directly across the membrane at the cathode side.

\subsection{Experimental}

\subsubsection{Membrane electrode assembly preparation}

The membrane electrode assembly (MEA) was prepared by a knife coating technique. $60 \% \mathrm{PtRu}(\mathrm{Hi}$ Spec 10.000$)$ and $60 \% \mathrm{Pt}(\mathrm{Hi}$ Spec 9.000) from Johnson-Mathey were used as anode and cathode catalyst. The catalyst was ultrasound dispersed in water, isopropanol, and Nafion solution to form a viscous ink. This viscous ink was then knife coated onto a carbon cloth GDL material and subsequently hot pressed $\left(135^{\circ} \mathrm{C}\right.$ for $\left.3 \mathrm{~min}\right)$ onto a Nafion 
117 membrane. The Pt metal loading on the cathode and anode were set to $3.5 \mathrm{mg} \mathrm{cm}^{-2}$ and $3 \mathrm{mg} \mathrm{cm}^{-2}$, respectively.

\subsubsection{Design of the in-situ fuel cell}

For spatially resolved XAS studies, a standard DMFC cell with $17.6 \mathrm{~cm}^{2}$ active area was modified by constructing X-ray windows at three different regions located relative to the fuel inlet and outlet (see Figure 4.1). This is achieved by removing the graphite from the flow fields at the region of interest and replacing it with Kapton. Further, to achieve the minimum perturbation in the temperature and electrical conductance, the graphite is preferentially removed from the channel regions of the flow field. The flow fields were also symmetrically drilled at both cathode and anode so that both transmission and fluorescence measurements were feasible. More technical details about the construction of the cell can be found elsewhere ${ }^{17}$.

\subsubsection{DMFC operation}

The DMFC was operated with a commercial portable fuel cell test bench from Magnum. In a typical DMFC single cell operation, $3 \mathrm{ml} / \mathrm{min}$ of $1 \mathrm{M}$ preheated methanol at $90{ }^{\circ} \mathrm{C}$ is pumped to the anode side and $150 \mathrm{ml} / \mathrm{min}$ of non-humidified oxygen is pumped to the cathode. The cell is externally heated to $60{ }^{\circ} \mathrm{C}$ and maintained at this temperature. In the DMFC, the fuel and oxidant inlets are constructed so that they are in "counter flow", i.e. the methanol inlet is positioned opposite the oxygen outlet and the methanol outlet opposite the oxygen inlet. In order to study the effect of oxygen flow rate on the adsorbate coverage, the DMFC was also operated at a reduced oxygen flow rate of $20 \mathrm{ml} / \mathrm{min}$. 


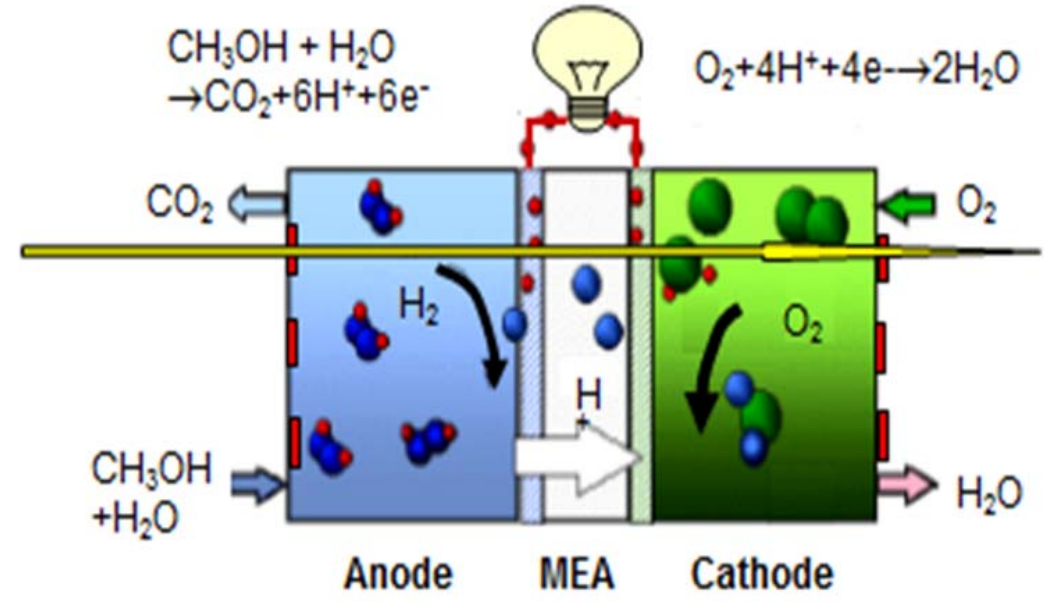

Figure 4.1 Methanol and $\mathrm{O}_{2}$ flow and the reactions occurring at each electrode in the DMFC studied in this work. 


\subsubsection{In-situ XAS measurements}

All XAS data at the $\mathrm{Pt} \mathrm{L}_{3}$ edge were recorded on the $\mathrm{X} 1$ beamline at Hasylab with a Si (111) monochromatized beam in both transmission and fluorescence geometry. Since Pt is present at both the anode and cathode, the transmission XAS data at the $\mathrm{Pt} \mathrm{L}_{3}$ edge of the DMFC is of little interest as it contains information from both the anode and cathode and cannot be separated. In such a scenario fluorescence measurements are preferred over transmission, as they can provide preferential information on the electrode facing the detector $^{18}$.

In-situ DMFC XAS measurements are often encountered with absorption spikes due to bubble formation from the oxidation of methanol. This issue is overcome in our present study by recording the spectra in QEXAFS mode with an acquisition time of $120 \mathrm{sec}$ over the complete $\mathrm{Pt} \mathrm{L}_{3}$ edge spectral range (11350-12560 eV). The QEXAFS fluorescence mode prevents the use of a 7 element energy resolved Ge detector due to its slow response time; instead in the present work a non-energy resolved PIPS diode was used to record the fluorescence data. A Zn filter is placed in front of the PIPS diode to screen any background scattering from the low atomic weight elements associated with the Kapton or carbon support. The cell with different window positions is aligned along the beam with a translational stage. Multiple QEXAFS spectra were measured at regions of interest on both the cathode and anode at various operating cell voltages for both the high and low oxygen flow rates. 


\subsection{5 $\Delta \mu$-XANES Technique:}

The IFEFFIT suite (Version 1.2.11 IFEFFIT Copyright 2008, Mathew Newville, University of Chicago, http://cars9.uchicago.edu/ifeffit) of programs was used for the $\Delta \mu$ XANES data analysis including the background subtraction (AUTOBK ${ }^{19}$ algorithm) and normalization $^{20}$. This $\Delta \mu$ technique has been described in detail elsewhere ${ }^{13-16}$. In summary, all raw spectra from different quick EXAFS scans were merged together for each single potential. To account for the drift in beam energy over the beam lifetime, these normalized foil spectra were then aligned to one standard foil spectrum. This energy correction is automatically transferred to the sample spectra as well. The success of the $\Delta \mu$ technique is very much dependent on the precision of this energy alignment step. A post-edge normalization procedure (30 to $200 \mathrm{eV}$ above edge, for $\Delta \mu$ - XANES analysis) is then applied to the sample spectra. The $\Delta \mu$ signatures are then obtained by subtracting the $\mu$ of a selected potential as reference;

$$
\Delta \mu=\mu(\mathrm{V})-\mu(\operatorname{Ref})
$$

The potential at which the electrode surface is the cleanest and has the least amount of adsorbates on it is utilized as reference. This reference potential has been chosen in the past to be around $0.54 \mathrm{~V}$ RHE in the absence of methanol and when water activation is at a minimum (i.e. when neither $\mathrm{H}$ or $\mathrm{O}$ is prevalent on the surface) ${ }^{13-14}$. However, in an operating fuel cell this "reference" potential has to be chosen more carefully ${ }^{15,16}$. At the anode, we chose XANES spectra taken on the same catalysts under nitrogen atmosphere at $0.5 \mathrm{~V}$ as reference. Under low $\mathrm{O}_{2}$ flow, consistent with procedures utilized in the past, we chose the highest current (lowest cell potential) as reference for the cathode spectra ${ }^{15,16}$. 
However under high $\mathrm{O}_{2}$ flow, we chose that potential which gave us a "positive" signature, i.e. that potential which gave us a $\Delta \mu$ signature of a representative adsorbate. These potentials can be seen as those points where the $\mathrm{OH}$ coverage or $\mathrm{OH}+\mathrm{CO}$ coverage is zero on the cathode. It should be emphasized that the coverage is not necessarily zero at this point, but rather the lowest throughout the potential range at that window.

To identify what the experimental $\Delta \mu$ signatures represent in adsorbate coverage, theoretical $\Delta \mu$ line shapes are constructed using the FEFF 8.0 code, exactly as reported previously $^{13-16}$. The theoretical $\Delta \mu$ spectra are constructed using the expression, $\Delta \mu_{\text {theo }}=\mu$ $\left(\mathrm{Pt}_{6} \mathrm{X}\right)-\mu\left(\mathrm{Pt}_{6}\right)$, where $\mathrm{X}$ is an adsorbate atom or group in a specific binding site (atop, bridged or $\mathrm{n}$-fold) and $\mathrm{Pt}_{6}$ is a six-Pt cluster previously described by Janin et al ${ }^{21}$. The M$\mathrm{M}$ bond distances $(\mathrm{M}=\mathrm{Pt}, \mathrm{Ru})$ used in FEFF 8.0 were $2.75 \AA, \mathrm{M}-\mathrm{C}=1.85 \AA, \mathrm{C}-\mathrm{O}=1.0 \AA$, $\mathrm{O}-\mathrm{H}=1.0 \AA$, and $\mathrm{M}-\mathrm{O}=2.0 \AA$. The theoretical $\Delta \mu$ signatures are usually shifted by 1-5 $\mathrm{eV}$, and scaled by a multiplication factor for ultimate comparison with the experimental data.

It should be emphasized that these theoretical $\Delta \mu$ are used only to identify the adsorbates responsible for the total observed experimental $\Delta \mu$, and often more than one adsorbate is on the surface simultaneously (e.g. $\mathrm{CO}$ and $\mathrm{OH}, \mathrm{CO}$ and $\mathrm{H}_{2} \mathrm{O}$, and even methanol). In this case, distinguishing features are chosen at some specific energy (such as those indicated in Figures 4.3 and 4.4) and the amplitude of these features are recorded to track the relative coverage of each adsorbate. 


\subsection{Results and Discussion}

\subsubsection{Estimation of anode and cathode potential}

Figure 4.2 shows the cell potential as a function of current for both $\mathrm{O}_{2}$ flow rates. One disadvantage of acquiring data on a working fuel cell is that most often only the cell potential is available, and therefore one does not know separately the anode and cathode potentials $^{22}$. This can be problematic for understanding the adsorbate coverage data, as generally the potential at the anode determines when water activation or methanol oxidation to $\mathrm{CO}$ occurs, and more specifically when water activation occurs on or near the $\mathrm{Ru}$ islands or away from them as described in the Introduction, Sec. 4.1. We have qualitatively drawn the anode and cathode potentials as a function of current in Figure 4.2 (it must be emphasized that these curves are indeed just qualitative) for the two $\mathrm{O}_{2}$ flow cases. They are indicated in Figure 4.2 to help illustrate a point; namely, that as the current increases (or cell potential decreases) the anode potential increases and the cathode potential decreases, but this change with current is dependent on conditions, such as the methanol and $\mathrm{O}_{2}$ flow rates. This plot also assumes that the local current density is relatively uniform over the area of the electrodes, a reasonable assumption at high $\mathrm{O}_{2}$ flow rate ${ }^{3}$ but not at low flow rate, as we will discuss further below. 


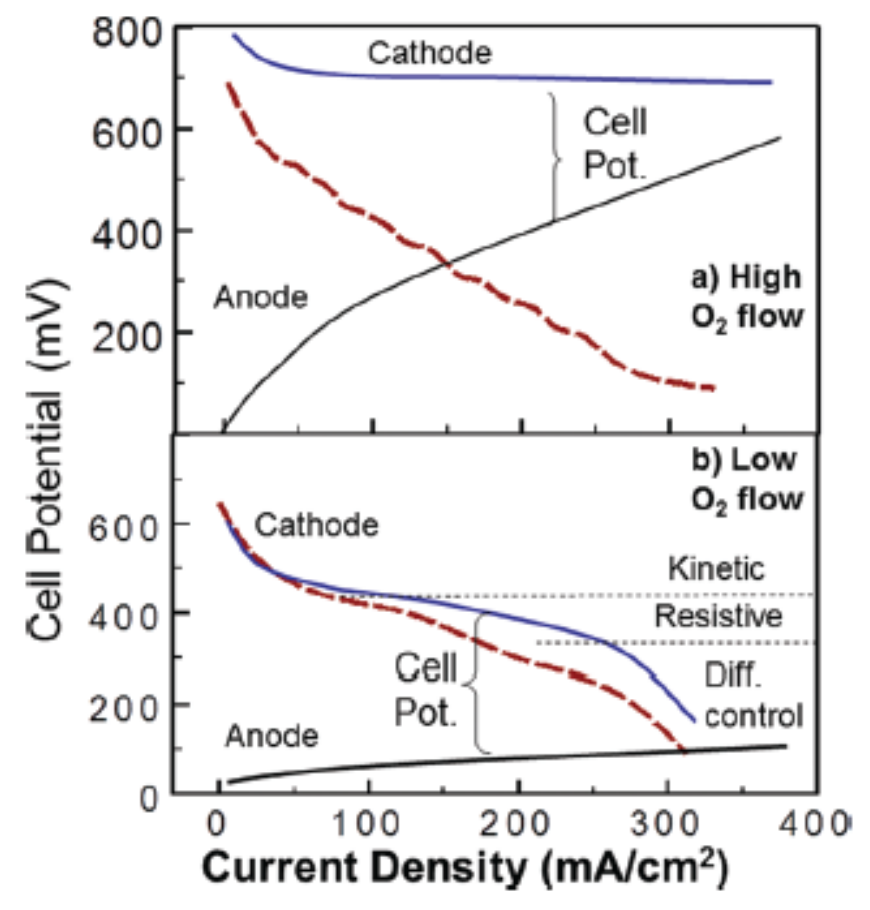

Figure 4.2 Plot of cell potential vs current density for the two different $\mathrm{O}_{2}$ gas flows as indicated. Also sketched are the estimated cathode and anode potentials. 
How much the anode potential increases over the cell potential range is determined in part by how much the cathode potential decreases over this same range. In a typical $\mathrm{H}_{2}$ fuel cell, the cell potential decreases with current primarily because of the cathode potential drop, since the kinetics at the $\mathrm{H}$ electrode are so fast ${ }^{23}$. This is indeed expected to be the case in the DMFC for low $\mathrm{O}_{2}$ flow rate, Figure 4.2b, when the different regimes (kinetic, resistive, and diffusion limitations) dominate at the cathode as illustrated and reported elsewhere ${ }^{24}$. However, in the DMFC with high $\mathrm{O}_{2}$ flow rate, it is anticipated that the largest fraction of the potential loss with current occurs at the anode, because now the kinetics of the methanol oxidation and resultant $\mathrm{CO}$ poisoning makes this process much slower than oxygen reduction. At very low currents or high cell potential, the anode potential is near zero, and the cathode potential must therefore be around $0.8 \mathrm{~V}$ as shown in Figure 4.2. The drop in the cathode potential depends on the "final" current or cell potential. A typical $\mathrm{H}_{2}$ fuel cell, operating with the same Pt loading as the cathode used in this work, would give currents of about $1000 \mathrm{~mA} / \mathrm{cm}^{2}$, or approximately 4 times larger than the final highest current shown in Figure $4.2^{26}$. Typical Tafel plots give a linear portion, with slope equal to $120 \mathrm{mV} /$ decade, only in the range between $700-500 \mathrm{mV}$, with diffusion limitations, $\mathrm{H}_{2} \mathrm{O}_{2}$ adsorption, and other factors causing the cathode potential then to start dropping with increased slope ${ }^{27}$. Considering the final current in Figure 4.2 is only $1 / 4$ that typically exhibited by a $\mathrm{H}_{2}$ fuel cell, we anticipate at most only a $100 \mathrm{mV}$ drop in the cathode potential at high $\mathrm{O}_{2}$ flow rate, and this occurs rather quickly with current. Assuming the cathode potential is then quite constant over most of the current range as shown in Figure 4.2a, the sum of the cell plus anode potential should then be around 700 
$\mathrm{mV}$. Thus the curves are qualitatively sketched in Figure $4.2 \mathrm{a}$ as shown, and the estimated anode potentials are shown at the top of Figure 4.5. We will give further arguments for the validity of these estimates in the next section. Again it must be emphasized that at low $\mathrm{O}_{2}$ flow rate, the electrode potentials will be very different (this will be discussed further below).

\subsubsection{High $\mathrm{O}_{2}$ flow result}

\subsubsection{1 $\Delta \mu$ results at the anode}

Figure 4.3 shows typical $\Delta \mu$ curves taken near the methanol inlet. These data are similar to that obtained for the middle and outlet regions. These curves have been obtained using the $\Delta \mu$-XANES procedures and reference spectrum described above. For comparison purposes, we show $\Delta \mu$ data from that reported previously by Scott et al ${ }^{15}$ along with FEFF8 calculated $\Delta \mu$ signatures reported there. Note that the present data is much less resolved; this arises because the present data is taken in a working fuel cell with relatively larger Pt loading so that the $\Delta \mu$ magnitudes are considerably smaller in magnitude and therefore contain significantly greater noise, and with a non-energy resolved PIPS detector for reasons stated above. They were also taken in Quick EXAFS mode (this to reduce $\mathrm{CO}_{2}$ bubble problems as explained above), but this also results in less signal to noise. To remove this noise, the data have been smoothed, which broadens out the features. While three

different $\mathrm{O}(\mathrm{H})$ features were clearly resolved in all of our previous reports ${ }^{15,16}$ with source as illustrated in Figure 4.3, we can only resolve the major $\mathrm{OH}$ feature now. The $\Delta \mu$ 
signature for $\mathrm{CO}$ is quite similar to that reported previously, except that some $\mathrm{O}(\mathrm{H})$ appears to be present even at this low anode potential (high cell potential).

In both Figures 4.3 and 4.4, shaded rectangles indicate the energy regions used to determine the magnitude of the $\Delta \mu$, denoted $|\Delta \mu|$ here, to reflect relative adsorbate coverage (i.e. lighted shaded rectangles reflect magnitude of $\mathrm{O}(\mathrm{H})$ coverage and dark shaded rectangles $\mathrm{CO}$ coverage). Unfortunately significant overlap exist between the $\Delta \mu$ signatures for $\mathrm{O}(\mathrm{H})$ and $\mathrm{CO}$, as shown by the theoretical FEFF8 signatures, so that no energy region truly reflects just one adsorbate. This overlap problem is particularly serious for the broad positive feature in the $\mathrm{CO}$ signature between 4 and $15 \mathrm{eV}$, as the $\mathrm{O}(\mathrm{H})$ signature has a significant negative component in this region. The experimental data at $630 \mathrm{mV}$ does not suggest any $\mathrm{O}(\mathrm{H})$ feature at $3 \mathrm{eV}$, so the $|\Delta \mu|_{\mathrm{CO}}$ magnitude is straightforwardly given from the zero axis. However, at 530 and $430 \mathrm{mV}$, significant $\mathrm{O}(\mathrm{H})$ is present, as indicated by the large positive feature at $3 \mathrm{eV}$, and such $\mathrm{O}(\mathrm{H})$ would produce a large negative component in the $4-15 \mathrm{eV}$ region (this is estimated by the black dashed line in Fig. 3). Therefore the dark shaded rectangles reflecting $|\Delta \mu|_{\text {CO }}$ is now estimated relative to this estimated black dashed line. The $|\Delta \mu|_{\mathrm{O}(\mathrm{H})}$ magnitude is always just estimated from the zero axis, since the experimental data at $630 \mathrm{mV}$ does not show a significant negative component around $3 \mathrm{eV}$, even though the $\mathrm{O}(\mathrm{H})$ coverage is expected to be quite small at this potential (i.e. the experimental $\Delta \mu_{\mathrm{CO}}$ signature does not appear to go as negative in this region as the FEFF8 theoretical signature suggests. 


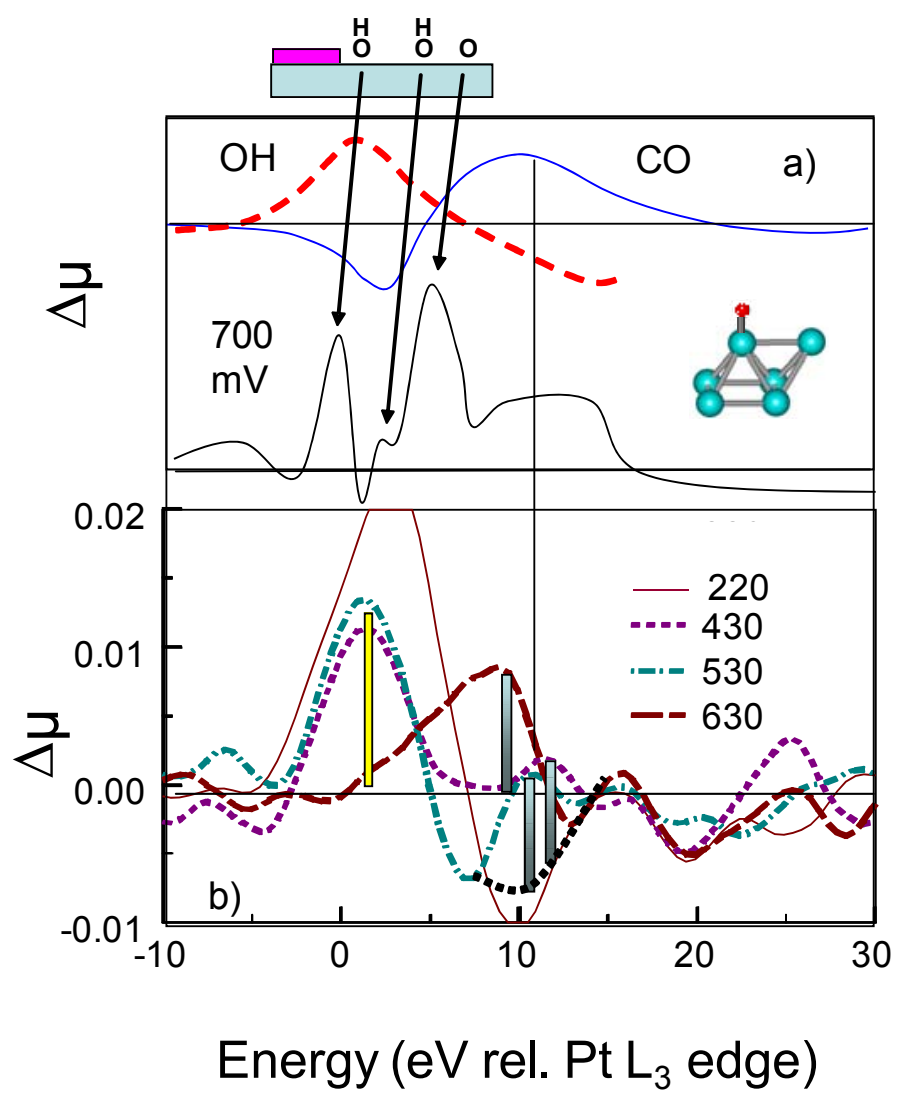

Figure 4.3:a) Theoretical signatures obtained from FEFF8 calculations for $\mathrm{OH}$ and $\mathrm{CO}$ in an atop site on a $\mathrm{Pt}_{6}$ cluster as illustrated. Also shown is $\Delta \mu$ data reported by Scott et al ${ }^{15}$ at the anode in methanol at $700 \mathrm{mV}$ illustrating the 3 separate peaks, coming from $\mathrm{OH}$ near and far from a Ru island and $\mathrm{O}$ on $\mathrm{Pt}$ as illustrated. b) Experimental data from the anode at the bottom window (near the methanol inlet) at the indicated cell potentials. Four shaded bars indicate $|\Delta \mu|$ magnitudes (light for $\mathrm{O}(\mathrm{H})$ and dark for $\mathrm{CO}$ ) utilized to prepare Figure 4.5 with base estimated as discussed in text. 


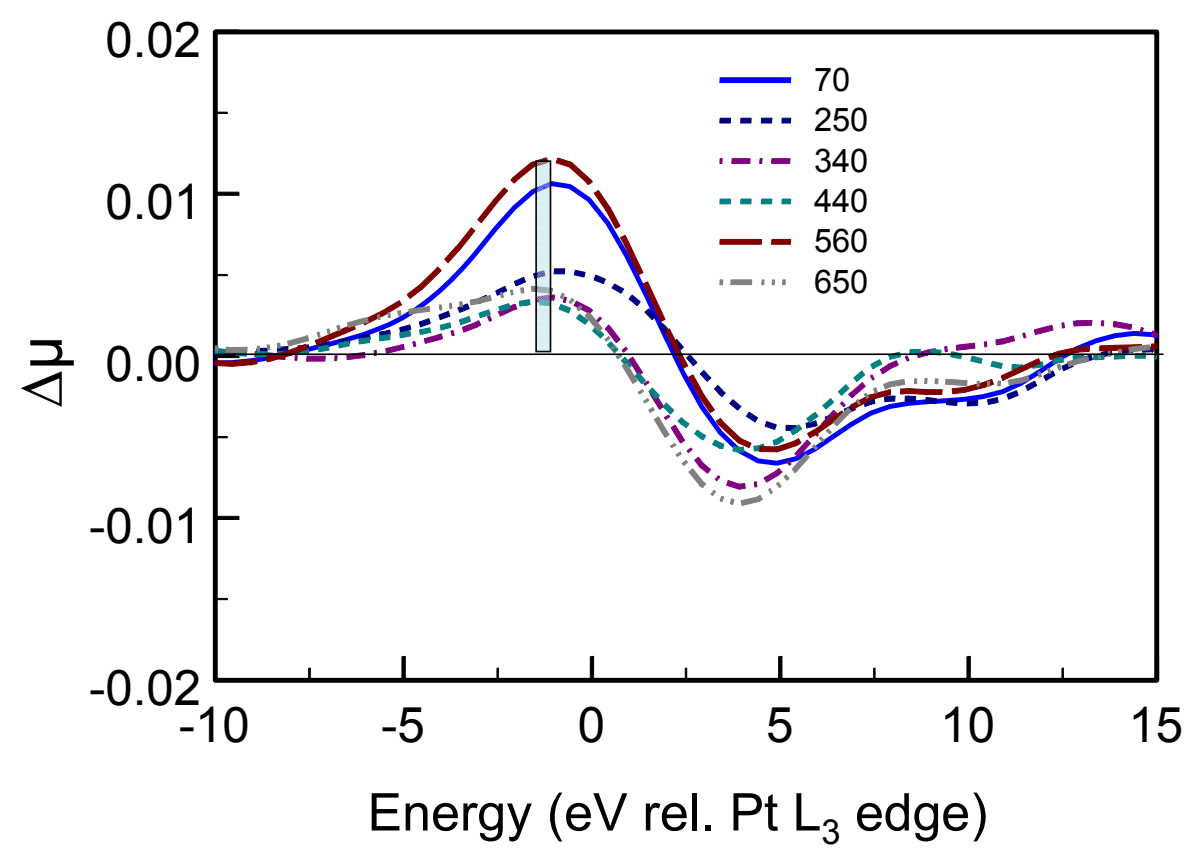

Fig. 4.4: Plot of $\Delta \mu$ at the indicated cell potentials (in $\mathrm{mV}$ ) at the cathode outlet with 200 $\mathrm{ml} / \mathrm{min} \mathrm{O}_{2}$ flow rate and using $150 \mathrm{mV}$ as the reference potential in the $\Delta \mu$ calculation. Note the oscillatory behavior of $|\Delta \mu|$ with potential. 
Figure 4.4 shows a plot of $\Delta \mu$ at the indicated cell potentials (in $\mathrm{mV}$ ) at the cathode outlet with $150 \mathrm{ml} / \mathrm{min} \mathrm{O}_{2}$ flow rate and using $150 \mathrm{mV}$ as the reference potential in the $\Delta \mu$ calculation. Again the light rectangle reflects relative $\mathrm{O}(\mathrm{H})$ coverage, always relative to zero. Note the apparent oscillatory behavior of $|\Delta \mu|_{\mathrm{O}(\mathrm{H})}$ with potential; it is large at 70 and $560 \mathrm{mV}$, but smaller at the other potentials. This will be discussed further below.

Figure 4.5 shows $|\Delta \mu|$ plots for the three different windows as a function of potential. For comparison purposes, data taken in an electrochemical cell for a $\mathrm{Pt}_{3} \mathrm{Ru}$ catalyst, prepared as described by Scott et al ${ }^{15}$ are also given. In our earlier work we defined 3 different potential regions named for the dominant $\mathrm{CO}$ oxidation mechanism, these mechanisms as described above (BF, DL, and D) differ only by the source of the $\mathrm{OH}$ which oxidizes the $\mathrm{CO}$. Comparison of the $\mathrm{CO}$ "coverage" at the methanol inlet varies somewhat similarly to what was found previously, and confirms that the anode potentials we estimate from the arguments in the previous section are reasonable.

Comparison of the $\mathrm{CO}$ coverage at the middle and methanol outlet at the top now shows the effects of decreased methanol concentrations. In the middle region, $\mathrm{CO}$ is found only in the DL region suggesting that the BF mechanism is totally able to limit the additional CO coming on at lower anode potentials because of the lower methanol concentration. Finally at the methanol outlet, no build-up of $\mathrm{CO}$ is evident, suggesting a still smaller methanol concentration is making it to the methanol outlet.

Finally, we should comment on the $\mathrm{OH}$ adsorption as suggested by the $|\Delta \mu|$. Recall that we cannot resolve the individual $\mathrm{OH}$ contributions coming from that near and distant 


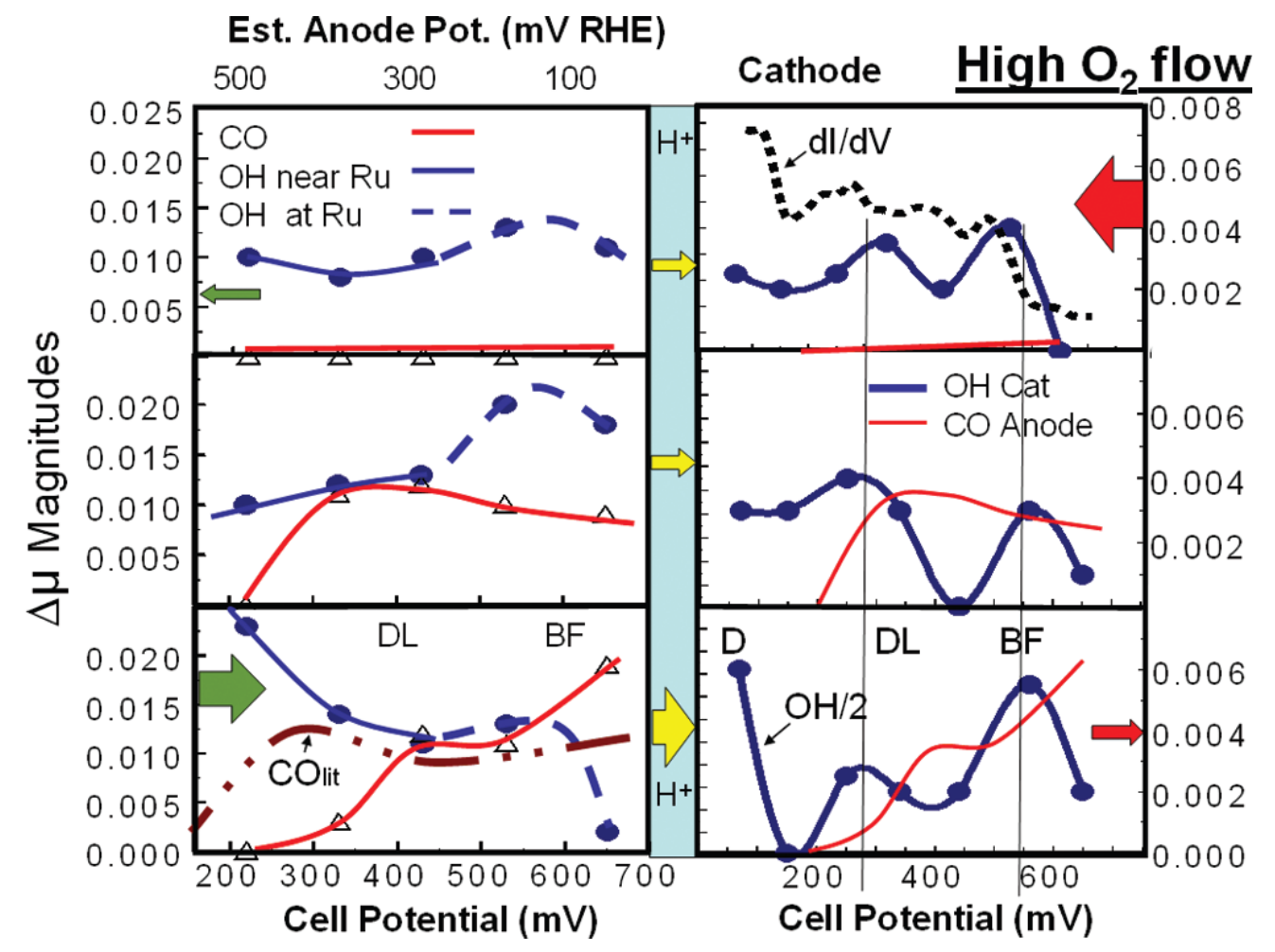

Figure 4.5: Plot of $|\Delta \mu|$ magnitudes vs cell potential for the indicated adsorbate species on the anode (left) and cathode (right) for the high $\mathrm{O}_{2}$ flow rate in the three separate windows. The relative adsorbate $\mathrm{CO}$ coverage on the anode is indicated also on the right to more directly show the correlation between anode and cathode coverages. The derivative of the cell current is also indicated at the cathode-top window. Finally "CO-lit" indicates the relative CO coverage found previously by Scott et al ${ }^{15}$ for methanol. Shaded arrows indicate the inlet/outlet for the methanol (green) and oxygen (red), and the relative magnitude of the $\mathrm{H}^{+}$transmission at each window (yellow). The symbols D (direct), DL (direct ligand) and $\mathrm{BF}$ (bifunctional) indicate the active $\mathrm{CO}$ oxidation mechanism as discussed in the Introduction. 
from the $\mathrm{Ru}$ like we were able in the earlier work ${ }^{15}$, but clearly the $\mathrm{OH}$ adsorption increases exactly where the $\mathrm{CO}$ coverage decreases, as one would expect.

The $\mathrm{OH}$ adsorption occurring already at $300 \mathrm{mV}$ anode potential is coming from water activation right at or even on the Ru islands, and that occurring at potentials greater than $300 \mathrm{mV}$ from that occurring on the $\mathrm{Pt}$ near the $\mathrm{Ru}$, and away from the $\mathrm{Ru}$. Three contributions will become even more evident in the cathode data. We have indicated the $\mathrm{OH}$ visible from the $\mathrm{Pt}$ that arises from water activation right at or on the $\mathrm{Ru}$ (i.e. via the BF mechanism) by the dashed line, and the remaining OH (DL or D) by the solid line.

\subsubsection{2. $\Delta \mu$ results at the cathode}

The right side of Figure 4.5 shows the corresponding $|\Delta \mu|$ magnitudes at the cathode. Before discussing these, we need to discuss the ORR reaction and the importance of $\mathrm{H}^{+}$to this reaction.

The kinetic equations derived from the "dissociative" double-trap kinetic model of Wang et al ${ }^{31,32}$ for the $4 \mathrm{e}^{-}$ORR in acidic media, include four elementary reactions:

$$
\begin{array}{ll}
1 / 2 \mathrm{O}_{2} \rightarrow \mathrm{O}_{\mathrm{ad}} & \text { Dissociative Adsorption (DA) (2) } \\
1 / 2 \mathrm{O}_{2}+\mathrm{H}^{+}+\mathrm{e}^{-} \rightarrow \mathrm{OH}_{\mathrm{ad}} & \text { Reductive Adsorption (RA) } \\
\mathrm{O}_{\mathrm{ad}}+\mathrm{H}^{+}+\mathrm{e}^{-} \rightarrow \mathrm{OH}_{\mathrm{ad}} & \text { Reductive Transition (RT) } \\
\mathrm{OH}_{\mathrm{ad}}+\mathrm{H}^{+}+\mathrm{e}^{-} \rightarrow \mathrm{H}_{2} \mathrm{O} & \text { Reductive Desorption (RD) }
\end{array}
$$

These reactions can be summarized even more basically as follows:

$$
1 / 2 \mathrm{O}_{2} \rightarrow \mathrm{OH}_{\mathrm{ad}}(\text { via RA or via } \mathrm{DA}+\mathrm{RT}) \rightarrow \mathrm{H}_{2} \mathrm{O}(\text { via } \mathrm{RD})
$$


and as shown by Wang et al, the RA mechanism is dominant below $0.7 \mathrm{~V}$ RHE. Thus the adsorbed $\mathrm{OH}$ intermediate is the most important. Although the exact ORR mechanism is still controversial, with some promoting a non-dissociative initial $\mathrm{O}_{2}$ adsorption ${ }^{32}$, the exact mechanism is not important here; the point is that virtually every step in the ORR requires $\mathrm{H}^{+}$and the best source of these $\mathrm{H}^{+}$may be those coming from the anode.

To illustrate the differences between operando results (i.e. when current is flowing and $\mathrm{H}^{+}$is coming through the PEM from the anode) vs the in situ equilibrium results (i.e. when current is not flowing), Figure 4.6 shows $\Delta \mu$ magnitudes on a cathode reflecting $\mathrm{O}(\mathrm{H})$ coverage in different environments ${ }^{33}$. Experiments were performed either in an electrochemical cell after saturating with $\mathrm{N}_{2}$ or $\mathrm{O}_{2}$ and the results compared to those from an in operando fuel cell. The in situ $\mathrm{N}_{2}$ results reflect the coverage of atop $\mathrm{OH}$ and fcc $\mathrm{O}$ due only to activation of water when moving the potential in the anodic direction. Below $0.7 \mathrm{~V}, \mathrm{OH}$ primarily exists on corners and edges of the Pt clusters, and above $0.7 \mathrm{~V}$ fcc $\mathrm{O}$ is observed. Saturating with $\mathrm{O}_{2}$, shows the additional effect of $\mathrm{O}_{a d}$ coming from reaction 2 above, causing the presence of $\mathrm{O}_{\mathrm{ad}}$ now beginning at $0.6 \mathrm{~V}$ and a slightly reduced coverage of $\mathrm{OH}$. The PEM fuel cell operando results, now obtained at the cathode, show a much larger coverage of $\mathrm{OH}$ extending down to well below $0.3 \mathrm{~V}$. To demonstrate that these results are consistent with the ORR kinetics explained above, we show theoretical model results from Wang et al ${ }^{30,31}$ using the double trap model in eqs. 2-5 for $\operatorname{Pt}(111)$. Unlike the experimental results, the model coverages are separated into $\mathrm{OH}$ and $\mathrm{O}$. From the model, a transition between atop $\mathrm{OH}$ and $\mathrm{O}$ is proposed at about $0.8 \mathrm{~V}$. In the experiment, however, transition points below $0.7 \mathrm{~V}$ are suggested. The higher transition point between the 


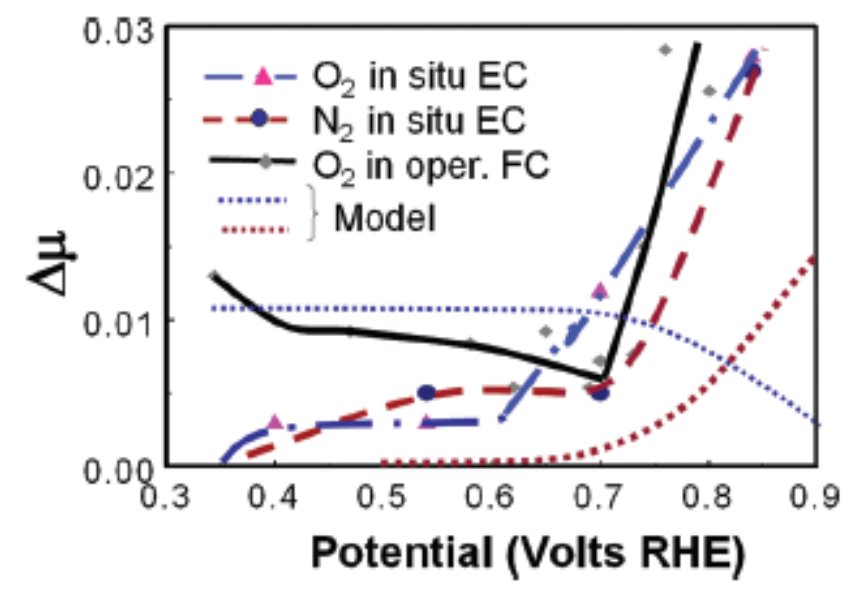

Figure 4.6: Plot of the previously reported $|\Delta \mu|$ after bubbling with $\mathrm{N}_{2}$ (only water activation) and $\mathrm{O}_{2}$ (water and $\mathrm{O}_{2}$ activation) in an electrochemical cell, and that for an operating fuel cell when full dynamic current is flowing ${ }^{33}$. Also shown is the $\mathrm{OH}$ (blue dotted) and $\mathrm{O}_{2}$ (red dotted) coverage (in arbitrary units) predicted by the Wang et al model for ORR on Pt(111) ${ }^{27,28}$. 
dominant $\mathrm{OH}$ vs. $\mathrm{O}$ coverage at $0.8 \mathrm{~V}$ is believed to reflect the lower reactivity of a $\mathrm{Pt}(111)$ surface compared to the 1-3 nm supported Pt particles, which have been used in the experiments. Otherwise the agreement in these results is remarkable, (the model results given in ML have been arbitrarily weighted to fit on the $\Delta \mu$ scale in Fig. 4.6). The increase in $\Delta \mu$ intensity below $0.3 \mathrm{~V}$ in the experimental PEMFC results is believed to arise from the adsorption of $\mathrm{H}_{2} \mathrm{O}_{2}$ (i.e. the breakdown in the 4-e ORR producing some 2-e ORR) due to the crowding of the surface from the adsorption of $\mathrm{H}_{2}$ and other anions at these low potentials $^{31}$.

The results immediately above suggest that the $\mathrm{OH}$ coverage on the cathode reflects in part the magnitude of the current flow; it increases as the current flow increases, or as the availability of $\mathrm{H}^{+}$coming across the PEM increases, because they should be proportional to each other. This is because more current or more $\mathrm{H}^{+}$increases the RA rate (eq.3) and this increases the $\mathrm{OH}$ coverage. However, the current is collected from all points on the fuel cell anode, but the $\mathrm{H}^{+}$comes across the PEM from the anode and should directly be reflected in what goes on at the cathode at the same window or observation point. This is particularly true if we think of the fuel cell as segments connected in parallel as assumed previously by Sauer et al $^{3}$.

The results in Figure 4.5 now confirm this "cross-talk" of $\mathrm{H}^{+}$transfer from anode to cathode. The $\mathrm{OH}$ adsorption on the cathode reflected in the $|\Delta \mu|$ each show two maxima at each window, with that at the bottom showing an additional feature at very low cell potential. To show that these track with the MOR going on at the anode, we re-plot the CO coverage on the anode on the right side with the cathode data. Careful inspection reveals 
that the $\mathrm{OH}$ coverage on the cathode peaks just as the $\mathrm{CO}$ coverage on the anode drops, exactly as one would expect since the current and $\mathrm{H}^{+}$yield is able to increase as the $\mathrm{CO}$ coverage drops. Thus the 3 features in the $\mathrm{OH}$ coverage on the cathode can be identified with the 3 different $\mathrm{CO}$ oxidation mechanisms identified above; namely the BF, DL, and D mechanisms named for the different sources of $\mathrm{OH}$ which oxidizes the $\mathrm{CO}$.

The "cross-talk" from $\mathrm{H}^{+}$transfer is also evident from the magnitudes of the $\mathrm{OH}$ anode coverage. Note that the $\mathrm{OH}$ coverage on the cathode bottom window has been divided by two in Figure 4.5 to fit it on the same scale. Therefore the largest current occurs at the bottom near the point of the methanol inlet, as would be expected. It is interesting that the magnitude of the $\mathrm{OH}$ coverage is similar at the middle and top observation windows, suggesting that the current flow is similar at these two points.

Finally, if the cathode $\mathrm{OH}$ coverage is indeed tracking with the $\mathrm{H}^{+}$cross-talk and electron current from the anode, the measured total current, although an integral of the current from each point on the electrodes, should track with the cell potential. To show this, we plot the derivative of the current with potential at the top of Figure 4.5 (i.e, the derivative $\mathrm{d} / \mathrm{dV}$ of the current plotted in Fig. 4.2). The derivative highlights the small features relative to the large overall slope coming from the large increase in current with cell potential. Again the features in this derivative fall at remarkably similar positions as the maxima in the cathode $\mathrm{OH}$ coverages. Note that two maxima in $\mathrm{d} / \mathrm{dV}$ fall in the $\mathrm{DL}$ region, but this is entirely consistent with the shift in the $\mathrm{OH}$ coverage at the top window vs. the middle and bottom windows. Thus the current appears to reflect all maxima in the $\mathrm{OH}$ coverage, one $\mathrm{D}$, two $\mathrm{DL}$, and the $\mathrm{BF}$ features. 


\subsubsection{Low $\mathrm{O}_{2}$ flow results}

Figure 4.7 shows the $\Delta \mu$ results for the lower $20 \mathrm{~mL} / \mathrm{min}_{2}$ flow rate. In order to understand the change in adsorbate coverages in this case, we first illustrate the expected changing anode and cathode potentials, in Figure 4.8. As suggested in our discussion of Figure 4.2, the cathode potential will now decrease more with cell potential, and consequently the anode potential increases less, but this change will vary with the window position, i.e. the extent of $\mathrm{O}$ starvation. As modeled by Sauer et al ${ }^{3}$, we can assume as illustrated in Figure 4.8 that the different paths or windows (top, middle, and bottom) of the electrode are connected in parallel, and because of the highly conducting end plates, the potential drop across each path is $\mathrm{V}_{\text {int }}=\mathrm{V}_{\text {open }}-\mathrm{V}_{\text {cell }}=\mathrm{V}_{\mathrm{c}}+\mathrm{V}_{\mathrm{a}}$, where the potentials are denoted with subscripts $(i n t=$ internal IR drop, open $=$ open circuit, cell $=$ measured cell, $\mathrm{a}=$ anode and $\mathrm{c}=$ cathode) and the internal IR drop consists of the sum of that at the anode and cathode. As the oxygen concentration decreases going from the top to the bottom window, the cathode potential will decrease faster with cell potential, because the $\mathrm{O}_{2}$ diffusion limit is reached at smaller currents (higher potential). In Figure 4. 8, we also show the expected local adsorbate coverage on the anode and cathode at the estimated local potential. The anode and cathode potentials are conveniently drawn linear with respect to cell potential. Of course this is not necessarily true, but Figure 4.8 is drawn only to emphasize the qualitative change in anode and cathode potentials with $\mathrm{O}_{2}$ concentration, and the predicted adsorbate coverages in each window region, not to accurately predict how these potentials change with cell potential. 


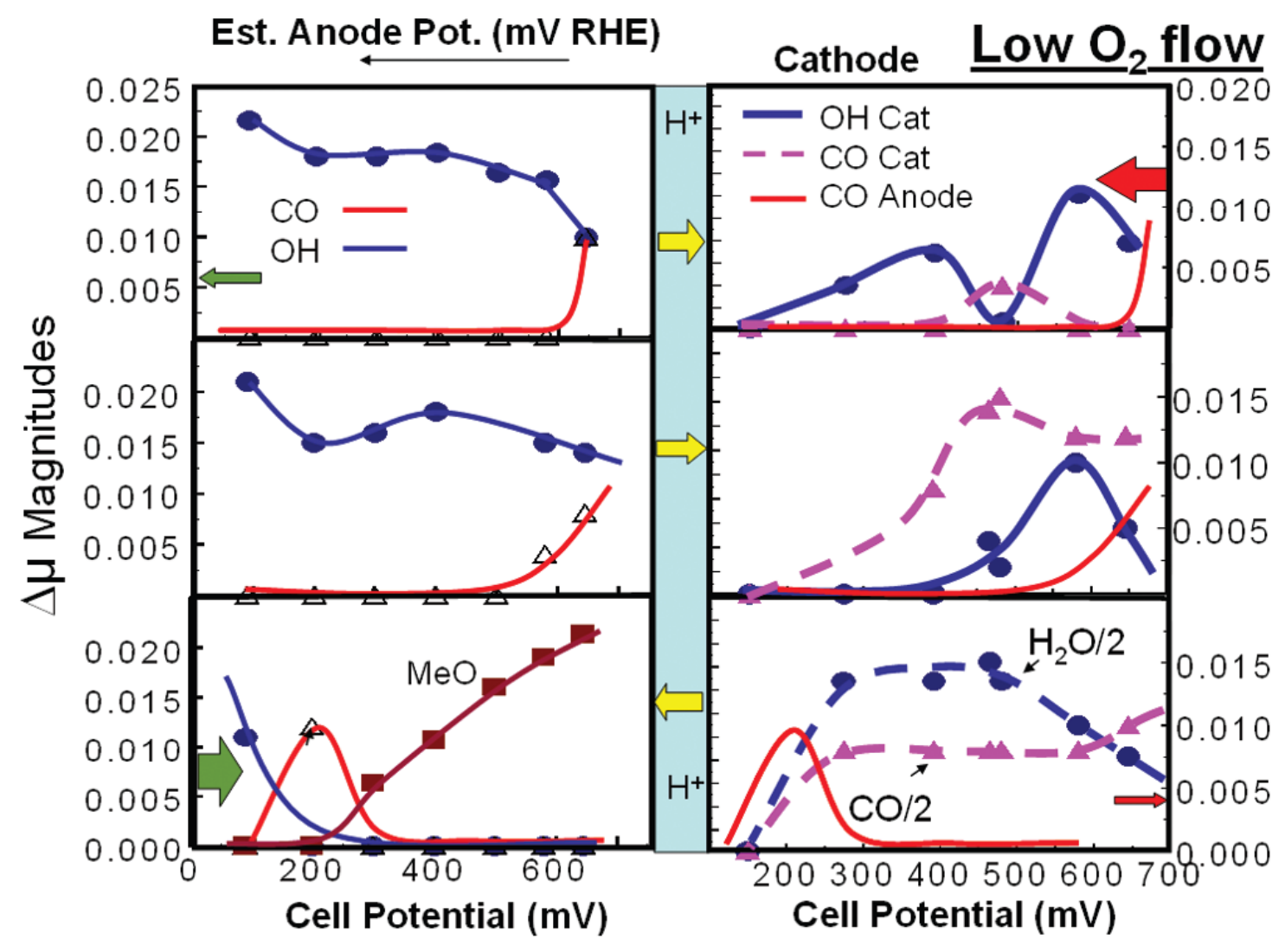

Figure 4.7: Exactly similar to Fig. 4.5 but now for low $\mathrm{O}_{2}$ flow rate. Note that the anode potential is now not give at the top because this now changes depending on the cathodic conditions as summarized in Figure 4.8. 
Figure 4.8 suggests that at the bottom window, the anode potential never gets much above $0.1 \mathrm{~V}$, but at the top window, it proceeds similar to that at $150 \mathrm{~mL} / \mathrm{min} \mathrm{O}_{2}$ flow. The predicted cathode and anode potentials drawn in Figure 4.2 are some effective average of these 3 "window" local potentials.

If the results at the lower $\mathrm{O}_{2}$ flow rate are going to be similar to those at the higher flow rate, this should occur at the top window near the $\mathrm{O}_{2}$ inlet, where the $\mathrm{O}_{2}$ concentration is the highest and the methanol concentration is probably still reasonable having survived because of $\mathrm{O}_{2}$ starvation and hence lower currents near the bottom. Indeed, Fig. 7 shows very similar results, showing the $\mathrm{CO}$ coverage on the anode dropping in the BF region with the $\mathrm{OH}$ coverage rising and the $\mathrm{OH}$ coverage on the cathode showing the two maxima at similar cell potentials ( 350 and $600 \mathrm{mV}$ ) similar to that for the high $\mathrm{O}_{2}$ flow rate. The fact that these so called BF and DL maxima fall at similar cell potentials found for the high $\mathrm{O}_{2}$ flow indeed suggest that the cell potential at the top window is governed primarily by the anode.

The $\mathrm{CO}$ coverage on the anode in the middle and bottom window, now reflect an increasing shift to lower cell potential (higher anode potential) for oxidation of the $\mathrm{CO}$, consistent with the expected shift in anode/cathode potential as depicted in Fig. 8. Indeed at the bottom window we see apparent methanol (or other oxidation intermediate) at all cell potentials above $200 \mathrm{mV}$, suggesting that the anode potential is below or near $100 \mathrm{mV}$ over this entire region. 


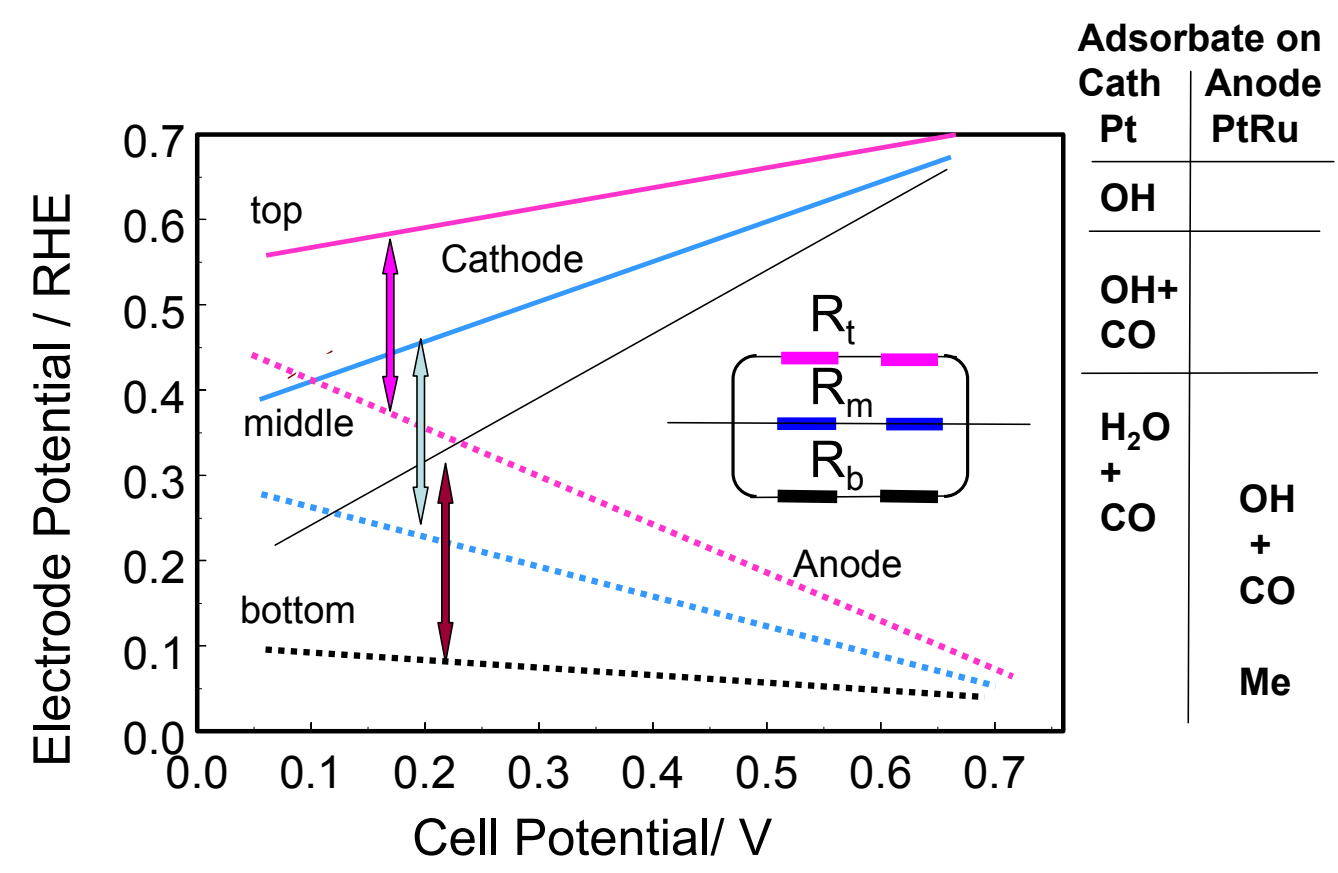

Figure 4.8 Qualitative estimates for cathode (solid) and anode (dotted) potentials as a function of cell potential at the top, middle, and bottom windows under low $\mathrm{O}_{2}$ flow conditions. Insert shows a model of segments (windows or resistors) connected in parallel. Also the estimated dominant adsorbates are indicated at the right for the corresponding electrode potential. 
On the cathode, in the middle window, and particularly in the lower window, very different behavior is evident. In both the middle and lower windows (and even a bit in the upper window), significant amounts of $\mathrm{CO}$ are found. This arises because methanol crossover is occurring, and the low $\mathrm{O}_{2}$ flow rate does not facilitate the flushing out of this methanol. This allows some of it to be oxidized resulting in $\mathrm{CO}$ or $\mathrm{C} 1$ (C1:- formaldehyde, formic acid, etc.) poisoning species on the cathode. Note that this $\mathrm{CO}$ appears with increasing coverage and to increasing cell potential as the $\mathrm{O}_{2}$ starvation sets in, consistent with the faster decrease in cathode potential with cell potential as schematically shown in Figure 4.8. The $\mathrm{OH}$ coverage on the cathode in the top and middle windows is consistent with the $\mathrm{CO}$ coverage on the anode, i.e., the $\mathrm{OH}$ coverage increases right when the $\mathrm{CO}$ coverage on the anode decreases, consistent with the high $\mathrm{O}$ flow results. Recall as above, this occurs because when the $\mathrm{CO}$ is decreasing on the anode, this enables more methanol oxidation, which produces more $\mathrm{H}^{+}$; these $\mathrm{H}^{+}$cross the PEM and enable faster ORR kinetics, thus increasing OH coverage on the cathode directly across the PEM. Very different adsorbate coverages are seen in the $\mathrm{O}_{2}$ starved region at the bottom window; with the adsorbate coverage large at the cell potentials when the methanol is on the anode. Here, we are probably observing $\mathrm{H}_{2} \mathrm{O}$ coverage instead of $\mathrm{OH}$, since the $\Delta \mu$ signatures of $\mathrm{OH}$ and $\mathrm{H}_{2} \mathrm{O}$ are the same. Previously we have seen adsorbed $\mathrm{H}_{2} \mathrm{O}$ at potentials just below those when $\mathrm{OH}$ becomes visible ${ }^{35}$; thus the identification of $\mathrm{H}_{2} \mathrm{O}$ and $\mathrm{CO}$ at this cathode potential in Figure 4.8. It would also be a bit unexpected to see large amounts of $\mathrm{OH}$ and CO because they should react to form $\mathrm{CO}_{2}$, thus we prefer the $\mathrm{H}_{2} \mathrm{O}$ assignment at the $\mathrm{O}_{2}$ outlet on the cathode. Finally large amounts of water may exist in this region pushed down 
by gravity from the regions further up and not flushed out of the cathode because of the low $\mathrm{O}_{2}$ flow.

Quite striking is the small coverage of all adsorbates at low cell potential on the cathode. The reference utilized to obtain the $\Delta \mu$ curves at the cathode was in all cases the $\mu$ at $200 \mathrm{mV}$ (highest current), so this by definition gives a zero coverage.

These results for low $\mathrm{O}_{2}$ flow dramatically reveal how now the cathode is dictating in part what occurs on the anode, i.e. the cross-talk is occurring in the reverse direction, as the cathode is now dictating the anode potential. The slow rise in anode potential with decreasing cell potential, as illustrated in Figure 4.8, does not allow oxidation of the methanol until at very low cell potential. Clearly the DMFC at the bottom window is operating "bifunctionally"; i.e. in the normal galvanic mode with protons going from the anode to the cathode at low cell potential and higher current, but electrolytically at higher cell potential and lower current, with methanol being oxidized at the cathode, and protons in this region proceeding in the opposite direction to produce $\mathrm{H}_{2}$ at the anode. This bifunctionality has been seen previously by Sauer et al $^{3}$, from measurement of the local current, but this is the first measurement of the adsorbates existing on the Pt anode and cathode in this electrolytic mode.

Kulikovsky et al ${ }^{11}$ have found that after bifunctional operation of the DMFC with reduced $\mathrm{O}_{2}$ feed, the DMFC actually performs better when the $\mathrm{O}_{2}$ flow rate is restored again. They show that both the anode and cathode are improved in the regions where the DMFC operated electrolytically, and suggest that the Pt cathode is cleaned by oxide removal, and the electrochemically active surface area (ECAS) of the anode is increased, 
brought about by the $\mathrm{H}_{2}$ evolution during electrolytic operation. This Pt cathode cleaning may explain the surprisingly large $\Delta \mu$ magnitudes in this bottom region, $\Delta \mu_{\mathrm{H} 2 \mathrm{O}}+\Delta \mu_{\mathrm{CO}} \sim$ 0.04 compared with about 0.02 in the top and middle regions, a factor of 2 larger. We cannot confirm, however, whether this increased $\Delta \mu$ total arises from simply higher adsorbate coverage in this bottom region, or an increased Pt surface area due to oxide removal during electrolytic operation.

\subsection{Summary and Conclusions}

In operando space resolved XAS measurements on a CCM anode and cathode during methanol oxidation through 3 different XAS windows at each electrode reveal significant variations in the adsorbate coverage over space and potential. A strong spatial dependence in adsorbate coverage, and therefore expected current density, relative to the inlet and outlet of methanol and $\mathrm{O}_{2}$ gases was found. The $\Delta \mu$ technique demonstrates the cross-talk between anode and cathode which was only observed in-directly in previous reports. This very strong "cross-talk" between the anode and cathode is seen with the anode dictating at high $\mathrm{O}_{2}$ flow rate the $\mathrm{OH}$ coverage on the cathode, resulting from the $\mathrm{H}^{+}$cross over to the cathode, and the cathode at low $\mathrm{O}_{2}$ flow rate dictating the $\mathrm{CO}$ oxidation on the anode via methanol and $\mathrm{H}^{+}$cross over. The electrode with the slowest rate (methanol oxidation at the anode or oxygen reduction at the cathode) dictates in part the local adsorbate coverage. To our knowledge these are the first results to show the direct correlation in space and potential of $\mathrm{OH}$ adsorbates on the cathode with $\mathrm{H}^{+}$production at the anode during the MOR reaction. It points directly to the importance of the $\mathrm{H}^{+}$supply coming across the membrane, 
even though the electrolyte is acidic and therefore contains significant $\mathrm{H}^{+}$ions. Historically

${ }^{36}$, the rate determining step (rds) has been attributed to the very first reductive adsorption step, but in the double trap mechanism of Wang et al ${ }^{30,31}$, this is not necessarily the case. This first report of the adsorbates existing on the Pt anode and cathode may also shed light on the ORR mechanism and kinetics and the importance of the $\mathrm{H}^{+}$concentration at the anode. Finally, the adsorbates found in the $\mathrm{O}_{2}$ starvation region consistently correlate with the previously reported spatially resolved current density measurements of Sauer, which show that a DMFC can go into bifunctional mode. In the $\mathrm{O}_{2}$ starved electrolytic region at high cell potentials, large amounts of $\mathrm{CO}$ and $\mathrm{H}_{2} \mathrm{O}$ were found on the cathode.

\section{Acknowledgements}

The kind support of Adam Webb, beamline scientist at X1 Hasylab, is gratefully acknowledged. Volker Loos and Gregor Hoogers from Kompetenzzentrum Brennstoffzelle, Umwelt-Campus Birkenfeld are acknowledged for the in-situ cell design and Klaus Wippermann, FZ Jülich is thanked for helpful discussions. Financial support from the BMBF under grant no. 03SF0324E is gratefully acknowledged. 


\section{5: References}

(1) Zhao, T.S.; Xu, C.; Chen, R.; Yang, W.W. Mass transport phenomena in direct methanol fuel cells. Progress in Energy and Combustion Science, 2009, 35, 275-292.

(2) Ye, Q.; Zhao, H.; Yang, J.; Prabhuran, Electrochemical Reactions in a DMFC under Open-Circuit Conditions. J. Electrochem. Solid-State Lett, 2005, 8, A52-A54.

(3) Sauer, D.U.; Sanders, T; Fricke, B.; Baumhofer, T.; Wipperman, K.; Kulikovsky, A.A.; Schmitz, H.; Mergel, J. Measurement of the current distribution in a direct methanol fuel cell-Confirmation of parallel galvanic and electrolytic operation within one cell. $J$. Power Sources, 2008, 176, 477-483.

(4) Lai, C.M.; Lin, J.C.; Hsueh, K.L.; Hwang, C.P.; Tsay, K.C.; Tsai, L.D.; Peng, Y.M. On the Accelerating Degradation of DMFC at Highly Anodic Potential J. Electrochem. Soc, 2008, 155, B843-B851.

(5) Schröder, A.; Wippermann, K.; Lehnert, W.; Stolten, D.; Sanders, T.; Baumhöfer, T.; Kardjilov, N.; Hilger, A.; Banhart, J.; Manke, I. The influence of gas diffusion layer wettability on direct methanol fuel cell performance: A combined local current distribution and high resolution neutron radiography study. J. Power Sources, 2010, 195, 4765-4771.

(6)Manke, I.; Harting, C.; Grunerbel, M,; Lehnert, W.; Kardojilov A.; Halbel, A.; Hilger,A.; Banhart, J.; Riesemeier, H. Investigation of water evolution and transport in fuel cells with high resolution synchrotron x-ray radiography. Appl. Phys. Lett, 2007, 90, 174105-174108.

(7) Ettinghausen, F.; Kleemann, J.; Michel, M.;Quintus, M.; Fuess, H., Roth, C. Spatially resolved degradation effects in membrane-electrode-assemblies of vehicle aged polymer electrolyte membrane fuel cell stacks. J. Power Sources, 2009, 194, 899-907.

(8) Kulikovsky, A.A.; Schmitz, H.; Wippermann, K.; Mergel, B.; Fricke, T.; Sanders, D.U.; DMFC: Galvanic or electrolytic cell? Electrochem. Commun, 2006, 8, 754-760.

(9) Mueller, H.; Dohle, A.A.; Kulikovsky, A.A. Comment on "Electrochemical Reactions in a DMFC under Open-Circuit Conditions" Electrochem. Solid-State Lett, 2006, 9, L7-L7.

(10) Schröder, A.; Wippermann, K.; Mergel, J.; Lehnert, W.; Stolten, D.; Sanders, T.; Baumhöfer, T.; Sauer, D.U.; Manke, I.; Kardjilov, N.; et al.; C. Combined local current distribution measurements and high resolution neutron radiography of operating Direct Methanol Fuel Cells, Electrochem. Commun, 2009, 11, 1606-1609. 
(11) Kulikovsky, A.A.; Schmitz, H.; Wippermann, K.; Mergel, J.; Fricke, B.; Sanders, T.; Sauer, D.U. Bifunctional activation of a direct methanol fuel cell. J. Power Sources, 2007, 173, 420-423.

(12) Park, S.M.; Kim, S.K.; Lim, S.; Jung, D.H.; Peck, D.H.; Hong, W.H. Experimental investigation of current distribution in a direct methanol fuel cell with serpentine flow-fields under various operating conditions, J. Power Sources, 2009, 194, 818-823.

(13) Teliska, M.; O’Grady, W. E.; Ramaker, D. E. Determination of H Adsorption Sites on $\mathrm{Pt} / \mathrm{C}$ Electrodes in $\mathrm{HClO}_{4}$ from $\mathrm{Pt} \mathrm{L}_{23} \mathrm{X}$-ray Absorption Spectroscopy. J. Phys. Chem $B, \mathbf{2 0 0 4}, 108,2333-2344$.

(14) Teliska, M.; O'Grady, W. E.; Ramaker, D. E. Determination of O and OH Adsorption Sites and Coverage in Situ on Pt Electrodes from Pt $\mathrm{L}_{23}$ X-ray Absorption Spectroscopy. J. Phys. Chem B, 2005, 109, 8076-8084.

(15) Scott, F.J.; Mukerjee, S.; Ramaker, D.E.; CO Coverage/Oxidation Correlated with PtRu Electrocatalyst Particle Morphology in $0.3 \mathrm{M}$ Methanol by In Situ XAS. $J$. Electrochem. Soc, 2007, 154, A396-A406.

(16) Scott, F.J.; Roth, C.; Ramaker, D.E. Kinetics of CO Poisoning in Simulated Reformate and Effect of $\mathrm{Ru}$ Island Morphology on PtRu Fuel Cell Catalysts As Determined by Operando X-ray Absorption Near Edge Spectroscopy. J. Phys. Chem. C, 2007, 111, 11403-11413.

(17) Dixon, D.; Schröder, A.; Schökel, A.; Söhn, M.; Manke, I.; Kardjilov, N.; Sanders, T.; Loos, V.; Hoogers, G.; Wippermann, K.; et al., MP Materials Testing, 2010, 10, 725-735.

(18) Roth, C.; Martz, N.; Mazurek, M.; Scheiba, F.; Fuess, H. Development of an InSitu Cell for X-ray Absorption Measurements During Fuel Cell Operation. Adv. Eng. Mat, 2005, 7, 952-956.

(19) Newville, M. IFEFFIT : interactive XAFS analysis and FEFF fitting, J. Synchrotron Rad, 2001, 8, 322-324.

(20) Newville, M.; Livina, P.; Yacoby, Y.; Stern, E.A.; Rehr, J. Near-edge X-ray-absorption fine structure of $\mathrm{Pb}$ : A comparison of theory and experiment,J. Phys. Rev. B, 1993, 47, 1412614131.

(21) Janin, E.; von Schenk, H.; Gothelid, M.; Karlsson, U.O. Phys. Rev. B, Bridgebonded atomic oxygen on Pt(110), 2000, 61, 13144-13149. 
(22) Roth, C.; Benker, N.; Buhrmester, T.; Mazurek, M.; Loster, M.; Fuess, H.; Koningsberger, D.C.; Ramaker, D.E. Determination of $\mathrm{O}[\mathrm{H}]$ and $\mathrm{CO}$ Coverage and Adsorption Sites on PtRu Electrodes in an Operating PEM Fuel Cell. J. Am. Chem. Soc., 2005, 127, 14607-14615.

(23) Gasteiger, H.A.; Kocha, S.S.;. Sompalli, B.; Wagner, F.T. Activity benchmarks and requirements for Pt, Pt-alloy, and non-Pt oxygen reduction catalysts for PEMFCs Appl. Catal. B: Environ, 2005, 56, 9-35.

(24) Larmanie, J.; Dicks, A. Fuel Cells Systems Explained, John Wiley \& Sons, 2000.

(25) Kim, J.; Lee, S.M.; Srinivasan, S.; Chamberlin, C.E. . Modeling of Proton Exchange Membrane Fuel Cell Performance with an Empirical Equation J. Electrochem. Soc, 1995, 142, 2670-2674.

( 26) Müller, J.; Frank, G.; Colbow, K.; Wilkinson, D. Transport/Kinetic Limitations and Efficiency Losses, In Handbook of Fuel Cells; John Wiley \& Sons, Ltd, D 2010.

(27) Wang, J.X.; Markovic, N.M.; Adzic, R.R., Kinetic Analysis of Oxygen Reduction on Pt(111) in Acid Solutions: Intrinsic Kinetic Parameters and Anion Adsorption Effects J. Phys. Chem. B, 2004, 108, 4127-4133.

(28) Vielstich, W.; Lamm, A.; Gasteiger, H. Handbook of Fuel Cells:Fundamentals, Technology, Applications; John Wiley \& Sons: Chichester, U.K., 2003.

(29) Wang, J.X.; Springer, T.E.; Liu, P.; Shao M.; Adzic, R.R. Hydrogen Oxidation Reaction on Pt in Acidic Media: Adsorption Isotherm and Activation Free Energies $J$. Phys. Chem. C, 2007, 111,12425-12433.

(30) Wang, J.X.; Zhang, J.; Adzic, R.R. Double-Trap Kinetic Equation for the Oxygen Reduction Reaction on $\operatorname{Pt}(111)$ in Acidic Media. J. Phys. Chem. A, 2007,111, 1270212710.

(31) Wang, J.X.; Uribe, F.A.; Springer, T.E.; Zhang, J.; Adzic, R.R. Intrinsic kinetic equation for oxygen reduction reaction in acidic media: the double Tafel slope and fuel cell applications. Faraday Discuss, 2009, 140, 347-362

(32) Rossmeisl, J.; Karlberg,G.S.; Jaramillob, T.; Nørskov, J.K. Steady state oxygen reduction and cyclic voltammetry, Faraday Discuss, 2008, 140, 337-346.

(33) Roth, C.; and Ramaker, D.E.; in Fuel Cell Science: Theory, Fundamentals, and Biocatalysis, Edited by Wieckowski, A.; and Nørskov, J.K. John Wiley \& Sons, 2010, Chap. 17, 511-541. 
(34) Ramaker, D.E.; Gatewood, D.; Korovina, A.; Garsany, Y.; Swider-Lyons, K.E. Resolving Sulfur Oxidation and Removal from $\mathrm{Pt}$ and $\mathrm{Pt}_{3} \mathrm{Co}_{2}$ Electrocatalysts Using in Situ X-ray Absorption Spectroscopy. J. Phys. Chem C, 2010, 114, 11886-11897.

(35) Yeager, E. Recent Advances in the Science of Electrocatalysis, J. Electrochem. Soc., 1981, 128, 160C- 171C.

(36) Yeager, E. Electrocatalysts for $\mathrm{O}_{2}$ reduction. J., Electrochim. Acta, 1984, 29, $1527-1537$. 


\section{Chapter 5: Super-iron Nanoparticles with Facile Cathodic Charge Transfer 5.1. Introduction}

$\mathrm{Fe}(\mathrm{VI})$ salts were named as super-irons in 1999 as they exist in thesuper-oxidized +6 valence state ${ }^{1}$. One ramification of this is that super-iron materials provide a multiple electron opportunity to store additional battery charge. Super-iron batteries have been explored including Li-ion super-iron batteries ${ }^{2,3}$ and alkaline super-iron batteries ${ }^{1,4}$ Charge storage has been accomplished via alkali super-iron salts, such as $\mathrm{Li}_{2} \mathrm{FeO}_{4}$, $\mathrm{Na}_{2} \mathrm{FeO}_{4}, \mathrm{~K}_{2} \mathrm{FeO}_{4}, \mathrm{Ru}_{2} \mathrm{FeO}_{4}, \mathrm{Cs}_{2} \mathrm{FeO}_{4}$, and mixtures or alloys of those salts ${ }^{2,4,5} . \mathrm{Fe}(\mathrm{VI})$ ferrates have been prepared by both chemical and electrochemical syntheses ${ }^{4,6}$. Alkaline earth super-iron salts, such as $\mathrm{BaFeO}_{4}$ and $\mathrm{SrFeO}_{4}$ have also performed as viable charge storage cathodic materials ${ }^{4,7}$, a transition metal super-iron salt, $\mathrm{Ag}_{2} \mathrm{FeO}_{4}{ }^{8}$ has been explored. The higher surface area to volume ratio of nanometer, compared to micron, size particles, should improve the sustainable current density. A decrease of particle size from the micrometer domain to the nanometer domain offers opportunities to facilitate charge transfer between battery materials, and thereby improve the power, voltage and depth of discharge. However, this decrease of particle size, which increases the ratio of surface area to volume, also exposes the particle to a greater risk of decomposition through enhanced chemical reactivity. Super-iron salts are fragile, reducing to Fe(III) with both heat and contact with water ${ }^{9}$, therefore making them unstable in the nanometer domain. In this paper an optimized pathway to decrease the super-iron particle size to the nano domain is introduced. X-ray absorption spectroscopy is utilized to study the oxidation state and structural changes of the nanoparticles and allows comparison with the 
discharge mechanism of larger particles. Once, stabilized, the nanoparticles could be coated with a zirconia overlayer, to prevent self-discharge as previously demonstrated on macroscopic super-irons ${ }^{9,10}$.

\subsection{Experimental}

$\mathrm{K}_{2} \mathrm{FeO}_{4}$ was chemically synthesized as reported ${ }^{11}$, recrystallized by dissolution in cold 2.67 M KOH, then filtered into cold $12 \mathrm{M} \mathrm{KOH}$, and stirred for $15 \mathrm{~min}$. The product was filtered, and washed in turn with hexane, isopropanol, methanol and ether, and then dried under vacuum for one hour. As seen in the upper SEM of Figure 1, with a Hitachi S-4700 high resolution scanning electron microscopy, the $\mathrm{K}_{2} \mathrm{FeO}_{4}$ product consists of micrometer domain particles. $\mathrm{K}_{2} \mathrm{FeO}_{4} \mathrm{IR}$ spectroscopy was measured with a PE Spectrum 100 FTIR with $\mathrm{KBr}$ and $\mathrm{BaSO}_{4}$ standard as described ${ }^{7,14}$. In order to more clearly observe the effect of the milling on the $\mathrm{K}_{2} \mathrm{FeO}_{4}$, X-ray absorption spectroscopy (XAS) data was taken at room temperature at the Fe $\mathrm{K}$ edge at the $\mathrm{X}-3 \mathrm{~B}$ beamline (National Synchrotron Light Source, Brookhaven National Laboratory, Upton, NY) with a Si (111) double crystal monochromator, and collected in fluorescence mode, with the sample placed between two gas ionization detectors and a Canberra 13-element Germanium solid state detector, which was collecting X-rays at a 90 degree angle with the incident beam. To account for the drift in the incident beam, a Fe reference foil ( $7 \mu \mathrm{m}$ thickness) was positioned between second and third ionization chamber detectors in all measurements. Super-iron primary cells were prepared in conventional $1.1 \mathrm{~cm}$ diameter A76 button cells. Non-milled and milled $\mathrm{K} 2 \mathrm{FeO} 4$ (with $10 \%$ carbon black) cathodic button cells were constructed and discharged with a $600 \Omega$ load. Cells were discharged to various 169 
percentages of the $3 \mathrm{e}-$ columbic efficiency, then opened, and the cathode composite removed, placed between two layers of Kapton tape, and studied with XAS. To determine the phase and morphology of the final discharge product, a variety of Fe (III) oxides ( $\alpha$ $-\mathrm{Fe}_{2} \mathrm{O}_{3}$ (Sigma Aldrich, MO, USA), $\gamma-\mathrm{Fe}_{2} \mathrm{O}_{3}$ (Sigma Aldrich, MO, USA), $\alpha-\mathrm{FeOOH}$ (Alpha Aesar, MA), $\gamma$-FeOOH (Alpha Aesar, MA, USA) and $\mathrm{Fe}_{3} \mathrm{O}_{4}$ (Pfaltz \& Buer, CT, USA) were also subject to ex-situ XAS measurements.

\subsection{Results and Discussion}

\subsubsection{Electrochemical Results}

$\mathrm{K}_{2} \mathrm{FeO}_{4}$, retains a solid state stability on the order of decades ${ }^{4,11,13}$. However, $\mathrm{K}_{2} \mathrm{FeO}_{4}$ does not sustain the high levels of electrochemical charge transfer as other less stable super iron salts, such as $\mathrm{BaFeO}_{4}{ }^{4,11,14}$. Previously, additives, such as silver or manganese salts, or an extended platinum black matrix, were needed to sustain high charge transfer rates in $\mathrm{K}_{2} \mathrm{FeO}_{4}{ }^{17-20}$, but a decrease in $\mathrm{K}_{2} \mathrm{FeO}_{4}$ particles to the nanometer domain presents a more direct opportunity to enhance the transfer rate.

Conventional, micron domain, $\mathrm{K}_{2} \mathrm{FeO}_{4}$ discharges effectively at low rates $(3000 \mathrm{ohm}$ discharge load over a $1 \mathrm{~cm}$ diameter alkaline $\mathrm{Zn}$ anode coin cell), when the $\mathrm{K}_{2} \mathrm{FeO}_{4}$ cathode contains high carbon additives (20\%) (Figure 5.2). The non-milled, $\mathrm{K}_{2} \mathrm{FeO}_{4}$ cathode cell, containing $20 \%$ carbon, when discharged at a low rate, exhibits a high discharge potential (1.6 V) (Figure 5.2). This cathode releases the majority of the three electron ( $\mathrm{Fe}(\mathrm{VI})$ to $\mathrm{Fe}(\mathrm{III}))$ theoretical capacity $\left(406 \mathrm{~mA} / \mathrm{g} \mathrm{K} 2 \mathrm{FeO}_{4}\right)$ available. However, the coulumbic efficiency and sustainable discharge voltage falls significantly (to $53 \%$ 


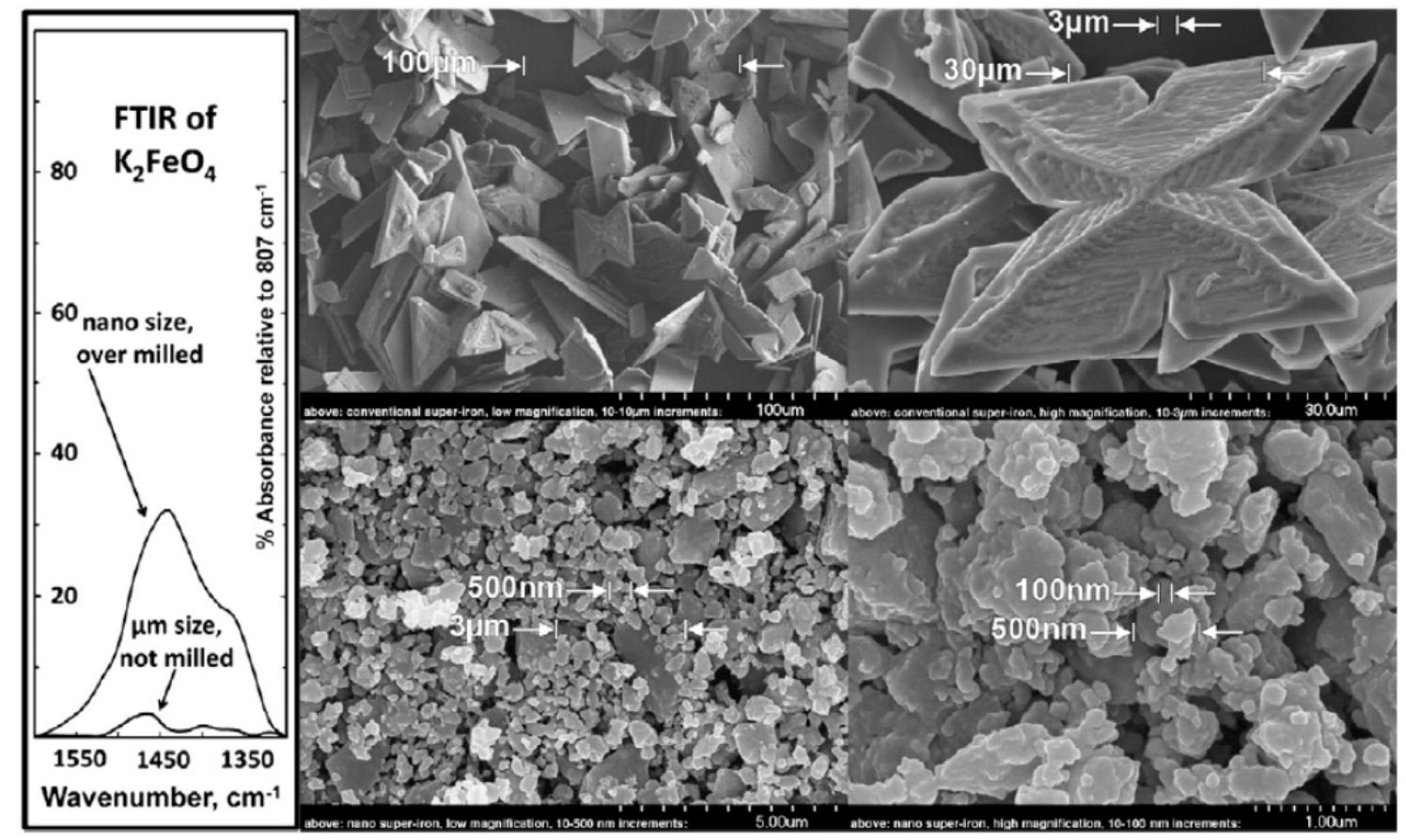

Figure 5.1 Left: $\mathrm{K}_{2} \mathrm{FeO}_{4}$ relative FTIR absorbance from $1300-1600 \mathrm{~cm}^{-1}$. Right: SEM of conventional and battery active nano $\mathrm{K}_{2} \mathrm{FeO}_{4}$. 


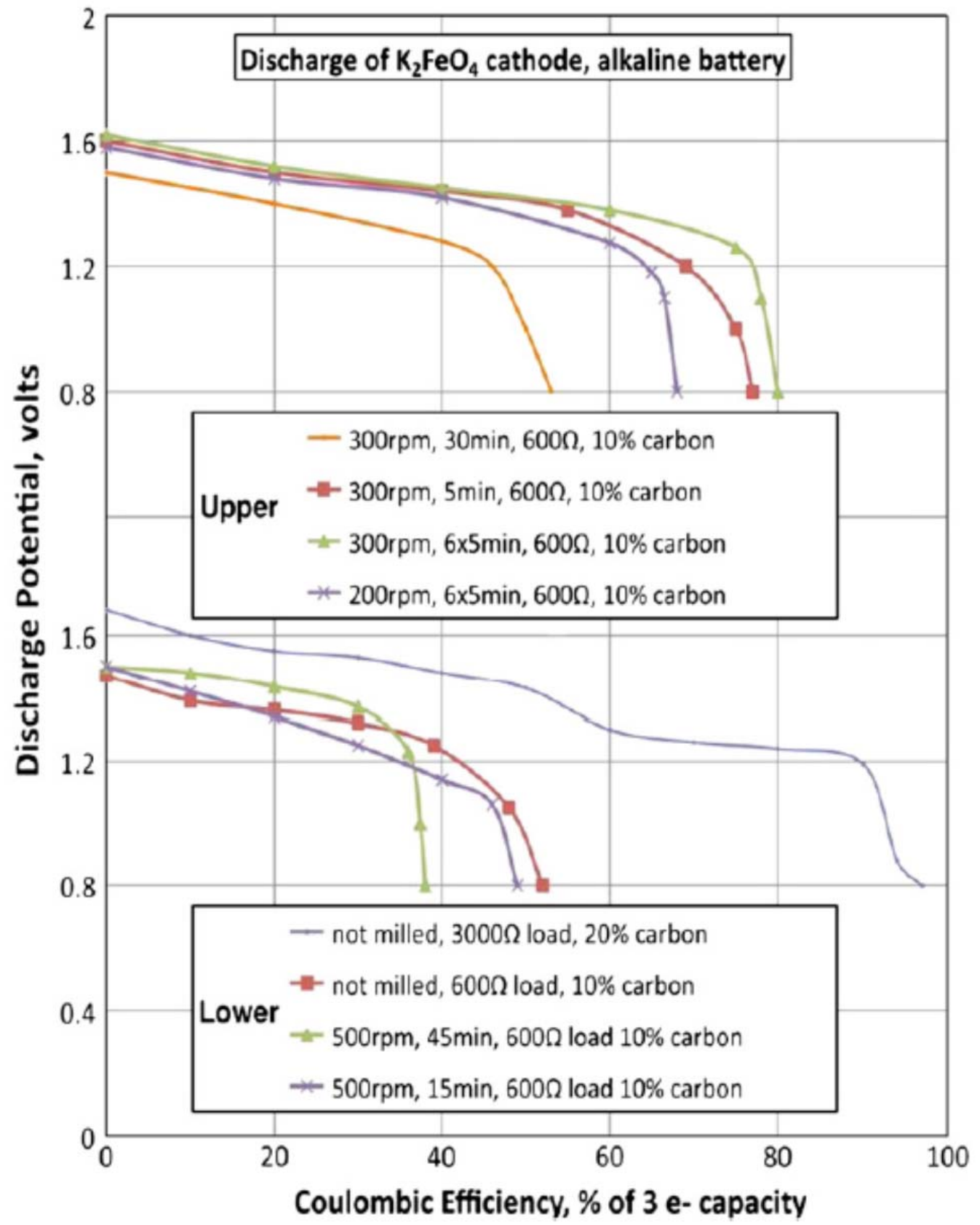

Figure 5.2 Discharge profile of super-iron batteries. 
depth of discharge to $0.8 \mathrm{~V}$ ) when the discharge rate of this non-milled $\mathrm{K}_{2} \mathrm{FeO}_{4}$ cell is increased (by decreasing the load resistor five-fold to $600 \mathrm{ohm}$ ) and when the carbon loading is decreased from $20 \%$ to $10 \%$ by weight. As previously discussed, Fe(III) salts can be highly insulating and their build-up, through chemical decomposition or inhomogeneous electrochemical discharge, can impede facile cathodic charge transfer ${ }^{16-}$ 20. We have not previously prepared stabilized submicron super-iron particles. Extended, aggressive ball milling is one of several techniques employed to decrease the particle size to the nanometer domain ${ }^{20,21}$.

However, ball milling can induce thermal decomposition of the sample. Chemically synthesized, recrystallized K2FeO4 was milled in a Retsch PM100 planetary ball mill with a steel vessel and steel grinding balls. As expected, aggressive ball milling of $\mathrm{K}_{2} \mathrm{FeO}_{4}$ heats the sample and converts some of the material to ferric (as evidenced by color change from purple black to rust, and a decrease in salt activity). Milling at $500 \mathrm{rpm}$ for 45 min does result in a higher discharge potential than the non-milled cathode cell at high rate (600 ohm load) (Figure 5.2). Yet there is a significant decrease in the activity of this ball milled $\mathrm{K}_{2} \mathrm{FeO}_{4}$ (with $10 \%$ added carbon) salt, and there is a decrease in coulombic efficiencies from $53 \%$ to $37 \%$. This ferric buildup, which occurs when $\mathrm{K}_{2} \mathrm{FeO}_{4}$ is aggressively ball milled is evident in the relative buildup of the Fe(III) peak at 1436 cm-1 (Figure 1). The principal IR absorption of $\mathrm{K}_{2} \mathrm{Fe}(\mathrm{VI}) \mathrm{O}_{4}$ occurs at $807 \mathrm{~cm}^{-1}{ }^{7}$, while that of $\mathrm{Fe}(\mathrm{VI})$ reduced to the ferric state occurs at $1436 \mathrm{~cm}-1^{14,15} \cdot \mathrm{K}_{2} \mathrm{FeO}_{4}$ absorbance is not significant from 1300 to $1600 \mathrm{~cm}-1^{7}$. Hence, the relative absorbance, of $\mathrm{A}(\mathrm{x}) /(\mathrm{A}(\mathrm{x})+\mathrm{A}(807 \mathrm{~cm}-1))$, for $\mathrm{x}=1300-1600 \mathrm{~cm}-1$, provides a measure of the $\mathrm{Fe}(\mathrm{III})$ 
buildup in the $\mathrm{K}_{2} \mathrm{FeO}_{4}$ sample. Figure 5.1 compares this for the chemically synthesized micron sized $\mathrm{K}_{2} \mathrm{FeO}_{4}{ }^{7}$ to that of the sample ball milled, continuously at $500 \mathrm{rpm}$ for 45 min. The decomposition of this aggressively ball milled sample is evident in the relative absorbance increase of the Fe(III) peak. This relative absorbance increase does not occur and the $\mathrm{K}_{2} \mathrm{FeO}_{4}$ peaks are fully retained when less aggressive ball milling is used as described below. The build-up of the Fe(III) product from discharge and chemical decomposition can also be followed with X-ray absorption and Mossbauer spectroscopy ${ }^{5,12,16}$. Here, we advance the X-ray analysis by simultaneously comparing EXAFS, which yields structural information, and XANES, which yields valence state information. This allows us to compare macroscopic versus stabilized nanoparticles as they undergo cathodic discharge from $\mathrm{Fe}(\mathrm{VI})$ to $\mathrm{Fe}(\mathrm{III})$ salts. Controlled, gentle, low heat planetary ball milling improves the electrochemical activity of mechano-synthesized $\mathrm{K}_{2} \mathrm{FeO}_{4}$. Discharge voltage increases and coulombic efficiency increases to $47 \%$ when the planetary ball mill time is shortened to $15 \mathrm{~min}$. Reducing the mill speed to $300 \mathrm{rpm}$, but increasing the mill time to $30 \mathrm{~min}$, further improves coulombic efficiency to $53 \%$ and improves discharge voltage relative to the sample milled at $500 \mathrm{rpm}$ for 15 or $45 \mathrm{~min}$.

At $300 \mathrm{rpm}$, but with a shorter $5 \mathrm{~min}$ mill, the discharge voltage increases and the coulombic efficiency substantially increases to $73 \%$. The optimal coulombic efficiency of $80 \%$, at $300 \mathrm{rpm}$, is observed when the sample is subjected to a $5 \mathrm{~min}$ mill, followed by 5 min cool down time, wherein this sequence is repeated 6 times. Reducing the rotational speed to 100 or $200 \mathrm{rpms}$, but retaining the same mill time/cool time sequence repeated 6 times, resulted in lower coulombic discharge efficiencies of $56 \%$ and $67 \%$. Decreasing 
the carbon black content of the optimal milled sample to $3 \%, 5 \%$, or $7 \%$ also resulted in lower coulombic efficiencies of $12 \%, 55 \%$, and $77 \%$ respectively.

As demonstrated in Figure 5.2, super-iron particles can be decreased to the nanometer domain, surprisingly without adversely impacting their utility as high capacity (3 electron) storage materials, and the small particle size increases the sustainable power density and cathodic charge transfer of these materials. This advance is made possible through an optimized process of less aggressive, dry and cool ball milling, and recognition of the specific phenomena (heat and water) which normally would prevent effective ball milling of super-irons. The decrease of the super-iron particle size is evident in the SEM results Figure 5.1, which compares the particle size of conventional (chemically synthesized) and nano (300 rpm ball milled with a six fold 5 min mill time/cool time sequence, in dry argon or nitrogen) nano $\mathrm{K}_{2} \mathrm{FeO}_{4}$. The majority of the conventional $\mathrm{K}_{2} \mathrm{FeO}_{4}$ particles range from 20 to $100 \mu \mathrm{m}$, whereas the optimized nano $\mathrm{K}_{2} \mathrm{FeO}_{4}$ particles range in size from 100 to $500 \mathrm{~nm}$. The optimized process forms particles which are not only 2 to 3 orders of magnitude smaller, but retain the high $3 \mathrm{e}^{-}$ storage capacity typical of active $\mathrm{Fe}(\mathrm{VI})$ cathode materials.

\subsubsection{XAS Results}

Comparison of the EXAFS signals of fully discharged samples and various ferric oxide and ferric hydroxide standards, showed that the final discharge product was primarily $\gamma-\mathrm{Fe}_{2} \mathrm{O}_{3}$. Most XAS spectra for the transition metals show a pre-edge peak due to the $1 \mathrm{~s}$ to $3 \mathrm{~d}$ excitation, appearing at lower energy than the $1 \mathrm{~s}$ to $3 \mathrm{p}$ dipole allowed excitation defined as the true edge (see inset of Figure 5.3$)^{22}$. The intensity of this peak 


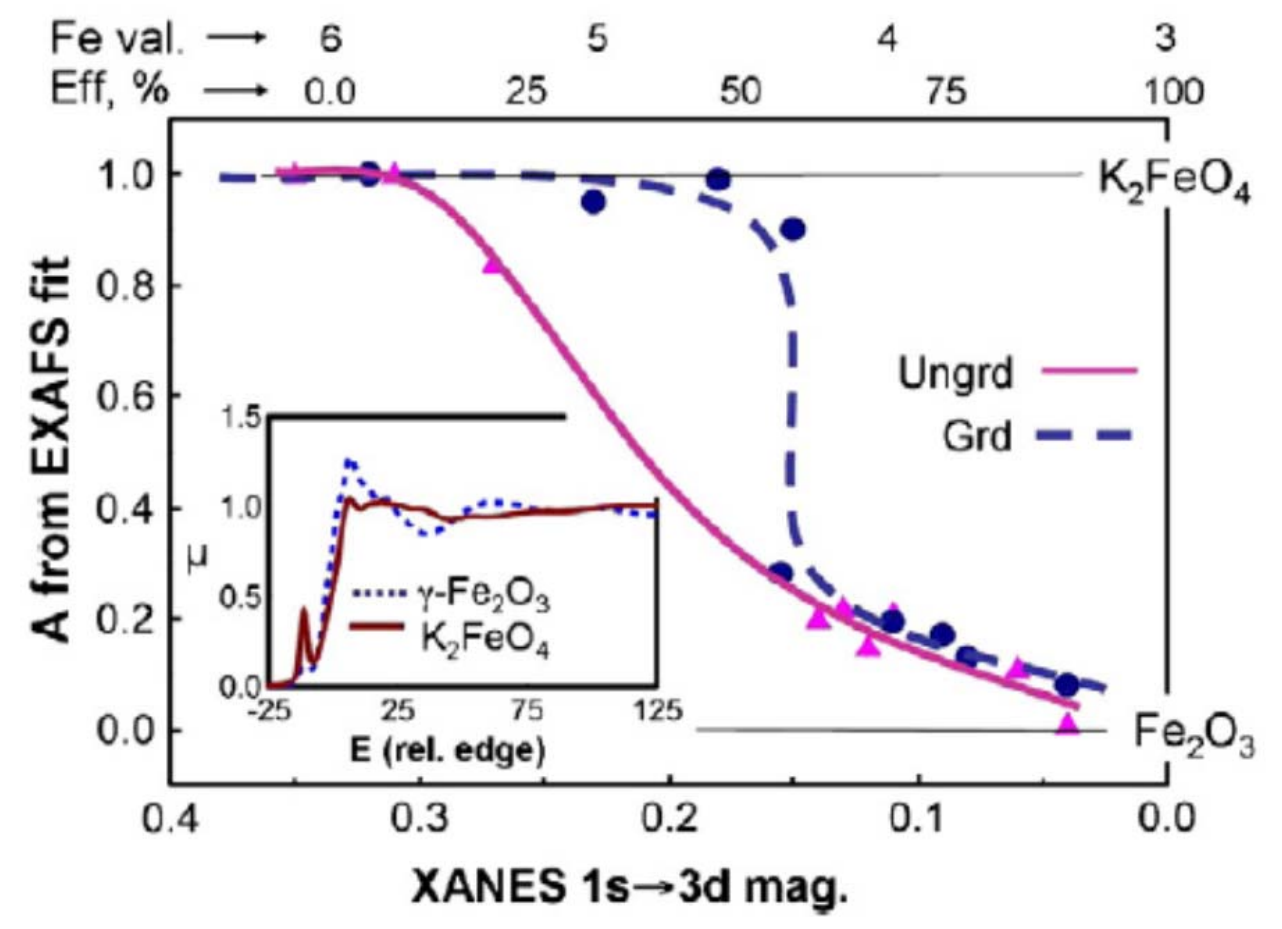

Figure 5.3 Plot of A from EXAFS fit vs. difference in 1s to 3d pre-edge peak; $\Delta \mu=\mu(\mathrm{t})$ $\mu(\gamma-\mathrm{Fe} 2 \mathrm{O} 3)$. Insert: plot of XAS data, $\mu(\mathrm{E})$ for the indicated samples, where the data for $\mathrm{K} 2 \mathrm{FeO} 4$ is for the undischarged milled cathode. 
generally increases with oxidation state as electrons are removed from the $\mathrm{d}$ shell. Thus it serves as a good measure of the Fe oxidation state, and the difference, $\Delta \mu=\mu(t)-\mu(\gamma-$ $\mathrm{Fe}_{2} \mathrm{O}_{3}$ ), in magnitude of this peak with time of discharge is plotted in Figure 5.3 to indicate oxidation level above that of $\gamma-\mathrm{Fe}_{2} \mathrm{O}_{3}$. In XAS, the extended X-ray absorption

fine structure above $50 \mathrm{eV}$ (EXAFS) is used to determine the structure immediately around the Fe atom. Here we fit the $\chi=\left[\mu(\mathrm{k})-\mu_{0}\right] / \mu_{0}$, obtained in the usual manner using the IFEFFIT (version http://cars9.uchicago.edu/ifeffit/) ${ }^{24}$

XAS analysis package, to the $\chi$ for the undischarged $\mathrm{K}_{2} \mathrm{FeO}_{4}$ cathode material and the discharge materials, $\gamma-\mathrm{Fe}_{2} \mathrm{O}_{3}$, i.e. $\chi(\mathrm{t})=\mathrm{A}^{*} \chi \mathrm{K}_{2} \mathrm{FeO}_{4}+(1-\mathrm{A})^{*} \chi \gamma-\mathrm{Fe}_{2} \mathrm{O}_{3}$. Figure 5.3 then shows the transition from the $\mathrm{K}_{2} \mathrm{FeO}_{4}$ structure with the usual $\mathrm{FeO}_{4}{ }^{2-}$ tetrahedral structure plus counter $\mathrm{K}^{+}$cations ${ }^{24}$, to the extended more insulating maghemite $\gamma-\mathrm{Fe}_{2} \mathrm{O}_{3}$ structure ${ }^{26}$ and accompanying $\mathrm{K}_{2} \mathrm{O}$. Figure 5.3 shows that this transition occurs rather suddenly around $55 \%$ of the discharge for the milled samples. For the non-milled sample the transition appears to occur gradually over the course of the entire discharge. The unground $\mathrm{K}_{2} \mathrm{FeO}_{4}$, with $10 \%$ carbon at $600 \mathrm{ohm}$ load, discharges to $53 \%$ coulombic efficiency (to an $0.8 \mathrm{~V}$ discharge cutoff) (Figure 2), which correlates with the majority conversion to $\mathrm{Fe}_{2} \mathrm{O}_{3}$ for this discharge in the Figure 5.3. Under these same discharge conditions, the optimized nano- $\mathrm{K}_{2} \mathrm{FeO}_{4}$ discharges to $80 \%$ coulombic efficiency (Figure 5.2). Under these conditions, the cathode retains its $\mathrm{Fe}(\mathrm{VI})$ structure through over $50 \%$ of the discharge, before undergoing a transition to the $\mathrm{Fe}_{2} \mathrm{O}_{3}$ structure (Figure 5.3). 


\subsection{Summary and Conclusions}

We have shown that a controlled, gentle, low heat planetary ball mill procedure considerably improves the electrochemical activity of mechano-synthesized $\mathrm{K}_{2} \mathrm{FeO}_{4}$, and that the discharge product is almost exclusively $\gamma-\mathrm{Fe}_{2} \mathrm{O}_{3}$. The nano-sized $\mathrm{Fe}(\mathrm{VI})$ cathodes can be discharged to nearly $55 \%$ of capacity without visible change in structure, while the micron-sized particles showed structural change already at $15 \%$ capacity. We have shown extended conductivity and reversibility of both the nonaqueous and alkaline $\mathrm{Fe}(\mathrm{VI})$ cathodes ${ }^{20,27}$. The nanoparticles allow for a more spatially uniform discharge with almost no change in structure until $\sim 50 \%$ discharge, and this suggested that partial discharge (50 to $70 \%$ ) might allow many more charge/discharge cycles.

\section{Acknowledgments}

We are grateful for the use of the X3-B and X-11A beam lines at the National Synchrotron Light Source, Brookhaven National Laboratory, which is supported by the U.S. DOE Office of Basic Energy Sciences, and partial support by NSF DMR award 1006568. 


\subsection{References}

(1) Licht, S.; Wang, B.; Ghosh, S. Energetic Iron (VI) Chemistry: The SuperIron Battery. Science 1999, 285, 1039.

(2) Licht, S. A High Capacity Li-Ion Cathode: The Fe(III/VI) Super-Iron Cathode. Energies 2010, 3, 960.

(3) Licht, S.; Wang, B. Nonaqueous Phase Fe(VI) Electrochemical Storage and Discharge of Super-Iron/Lithium Primary Batteries. Electrochem. Solid State Lette. 2000, 3, 209.

(4) Licht, S. Super-Iron Batteries. Encyclopedia of Electrochemical Power Sources 2009, 4, 262-284.

(5) Licht, S.; Naschitz, V.; Rozen, D.; Halperin, N. Cathodic Charge Transfer and Analysis of $\mathrm{Cs} 2 \mathrm{FeO} 4, \mathrm{~K} 2 \mathrm{FeO} 4$, and Mixed Alkali Fe,,VI... Ferrate Superirons. J. Electrochem. Soc. 2004, 151, A1147-A1151.

(6) DeKoninck, M.; Brousse, T.; Belanger, D. The electrochemical generation of ferrate at pressed iron powder electrodes: effect of various operating parameters. Electrochim. Acta 2003, 48, 1425-1433.

(7) Licht, S.; Naschitz, V.; Ghosh, S.; Lin, L.; Lui, B. SrFeO 4 : Synthesis, Fe(VI) characterization and the strontium super-iron battery. Electrochem. Commun. 2001, 3, 340-345.

(8) Licht, S.; Yang, L.; Wang, B. Synthesis and analysis of $\mathrm{Ag}_{2} \mathrm{FeO}_{4} \mathrm{Fe}(\mathrm{VI})$ ferrate super-iron cathodes. Electrochem. Commun. 2005, 7, 931-936.

(9) Licht, S.; Yu, X.; Wang, Y. Stabilized Alkaline Fe (VI) Charge Transfer: The Zirconia Coating Stabilized Superiron Alkaline Cathode. J. Electrochem. Soc. 2008, 155, A1-A7.

(10) Licht, S.; Yu, X.; Zheng, D. Cathodic chemistry of high performance Zr coated alkaline materials. Chem. Comm. 2006, 41, 4341.

(11) Licht, S.; Naschitz, V.; Ghosh, S.; Lui, B.; Halperin, N.; Halperin, L.; Rozen, D. Chemical synthesis of battery grade super-iron barium and potassium Fe(VI) ferrate compounds. J. Power Sources 2001, 99, 7-14.

(12) Licht, S.; Naschitz, V.; Halperin, L.; Halperin, N.; Lin, L.; Chen, J.; Ghosh, $\mathrm{S}$; Liu, B. Analysis of ferrate(VI) compounds and super-iron $\mathrm{Fe}(\mathrm{VI})$ battery 179 
cathodes: FTIR, ICP, titrimetric, XRD, UV/VIS, and electrochemical characterization. J. Power Sources 2001, 2, 167-176.

(13) Licht, S.; Tel-Vered, R. T.; Halperin, L. Toward Efficient Electrochemical Synthesis of $\mathrm{Fe}(\mathrm{VI})$ Ferrate and Super-Iron Battery Compounds. J. Electrochem. Soc. 2004, 151, A31.

(14) Licht, S.; Naschitz, V.; Wang, B. Rapid chemical synthesis of the barium ferrate super-iron Fe (VI) compound, $\mathrm{BaFeO}_{4}$. J. Power Sources 2002, 109, 67-70.

(15) Ghosh, S.; Wen, W.; Urian, R. C.; Heath, C.; Srinivasamurthi, V.; Reiff, W. M.; Mukerjee, S.; Naschitz, V.; Licht, S. Reversible Behavior of

$\mathrm{K}{ }_{2} \mathrm{Fe}$ ( VI ) $\mathrm{O}_{4}$ in Aqueous Media: In Situ Mössbauer and Synchrotron X-Ray Spectroscopy Studies. Electrochem. Solid State Lett. 2003, 6, A260.

(16) Licht, S.; Ghosh, S.; Naschitz, V.; Halperin, N.; Halperin, L. Fe(VI) Catalyzed Manganese Redox Chemistry: Permanganate and Super-Iron Alkaline Batteries. J. of Phys. Chem. B 2001, 105, 11933-11936.

(17) Licht, S.; Naschitz, V.; Ghosh, S. Silver Mediation of Fe(VI) Charge Transfer: Activation of the $\mathrm{K}_{2} \mathrm{FeO}_{4}$ Super-iron Cathode. J. Phys. Chem. B 2002, $106,5947-5955$.

(18) Licht, S.; DeAlwis, C. Conductive-Matrix-Mediated Alkaline Fe(III/VI) Charge Transfer: Three-Electron Storage, Reversible Super-Iron Thin Film Cathodes. J. Phys. Chem. B2006, 110, 12394-12403.

(19) Licht, S.; Wang, Y.; Gourdin, G. Enhancement of Reversible Nonaqueous Fe(III/VI) Cathodic Charge Transfer. J. of Phys. Chem. C 2009, 113, 9884-9891.

(20) Teja, A. S.; Koh, P. Y. Synthesis, properties, and applications of magnetic iron oxide nanoparticles. Progress in Cryst. Growth Charac. Mater. 2009, 55, 22-45.

(21) Cuenya, B. R. Synthesis and catalytic properties of metal nanoparticles: Size, shape, support, composition, and oxidation state effects. Thin Solid Films 2010, $518,3127-3150$.

(22) Shulman, R. G.; Yafet, Y.; Eisenberger, P.; Blumberg, W. E. Observations and interpretation of x-ray absorption edges in iron compounds and proteins P. Natl. Acad. Sci. USA. 1976, 73, 1384-1388. 
(23) Newville, M. IFEFFIT : XAFS analysis and FEFF fitting. J. Synchrotron Radiation 2001, 8, 322-324.

(24) Li, C.; Li, X. Z.; Graham, N. A study of the preparation and reactivity of potassium ferrate. Chemosphere 2005, 61, 537-543.

(25) Pinney, N.; Kubick, J. D.; Middelmiss, D.; Grey, C. P.; Morgan, D. Density Functional Theory Study of Ferrihydrite and Related Fe-Oxyhydroxides. Chem Mater, 2009, 21, 5727-5742.

(26) Kiltypin, M.; Licht, S.; Nowik, I.; Tel-Vered, R. T.; Levi, E.; Gofer, Y.; Aurbach, D. Study of Various ("Super Iron") $\mathrm{MFeO}_{4}$ Compounds in Li Salt Solutions as Potential Cathode Materials for Li Batteries. J. Electrochem. Soc. 2006, 153, A32-A41. 


\section{Chapter 6: Studying the Reversibility of Multi-electron Charge Transfer in Fe(VI) Cathodes Utilizing X-ray Absorption Spectroscopy}

\subsection{Introduction}

The sharp increase in energy demands of modern portable electronics devices, such as laptops, and the critical need in future electric automobiles, necessitates the search for new materials that can potentially offer higher storage capacities beyond that of current Li ion batteries. ${ }^{1}$ Current lithium and lithium-ion anode batteries are limited by the cathode capacity, as the anodes generally have higher capacity and suffer less potential loss with capacity. Therefore, there is a vital need for higher capacity cathode materials. Other important characteristics that an improved cathodic material should possess are, stability, facile charge transfer, high oxidative electrochemical potential, and low solubility in the electrolyte; attributes which would allow long battery life and high energy output. ${ }^{2}$

The most common cathode materials for secondary and primary batteries utilize redox reactions that undergo a single (or partial) electron transfer per redox atom within their working potential. Cathode materials that can offer multi-electron charge transfer generally offer higher energy densities. Hexa-valent iron salts, or "super-iron" salts,

contain highly oxidized iron $\left(\mathrm{Fe}^{6+}\right)$ that can theoretically undergo a three electron reduction to $\mathrm{Fe}^{3+}$ in a relatively narrow electropositive cathodic potential range, theoretically offering intrinsic capacities much higher than current cathodic compounds 
(e.g., $406 \mathrm{mAh} / \mathrm{g}$ for $\mathrm{K}_{2} \mathrm{FeO}_{4}$ compared to $274 \mathrm{mAh} / \mathrm{g}$ for commercially available $\mathrm{LiCoO}_{2}$, calculated as the intrinsic number of Faradays normalized by the molecular weight). Furthermore, the resultant $\mathrm{Fe}^{3+}$ discharge product is environmentally benign. A main challenge associated with the super-iron salts is that the $\mathrm{Fe}^{3+}$ discharge product (nominally $\left.\mathrm{Fe}_{2} \mathrm{O}_{3}\right)^{6}$ is highly resistive impeding reversible charge transfer., ${ }^{3,4}$

To alleviate the poor reversibility issue with super-iron salts, various approaches have been taken to either provide a more conductive path or a reduced charge carrier path length. Thin films of the $\mathrm{Fe}(\mathrm{VI})$ salt have been electrochemically deposited on a conductive matrix such a smooth Pt surface or on a "platinized" rough surface. These show relatively high reversibility in both aqueous and non-aqueous solvents. ${ }^{4,5}$ Recently, a new low-heat and gentle mechano-synthesized (ball mill) approach was taken to reduce the micron super-iron particle size to the nano domain. The decreased particle size can potentially offer a higher reversibility by decreasing the electron and/or ion path length as well. ${ }^{6}$ In highly basic aqueous $\mathrm{KOH}$ electrolyte, the reversibility of $\mathrm{Fe}(\mathrm{VI})$ is attributed to a hydrogen intercalation mechanism. ${ }^{7}$ For $\mathrm{K}_{2} \mathrm{FeO}_{4}$ the reversible reaction can be written as:

$\mathrm{K}_{2} \mathrm{FeO}_{4}+\mathrm{xH}_{2} \mathrm{O}+\mathrm{xe}^{-} \rightleftarrows \mathrm{H}_{\mathrm{x}} \mathrm{K}_{2} \mathrm{FeO}_{4}+\mathrm{x} \mathrm{OH}^{-}$

Therefore increased reversibility requires both a more conductive path for the electrons as well as for the protons, which move in and out of the ferrate salt matrix with charge/discharge. 
Previously the chemistry of the three-electron redox $\mathrm{Fe}(\mathrm{VI}) / \mathrm{Fe}(\mathrm{III})$ couple has been studied by various electrochemical techniques and a limited number of spectrochemical techniques; namely, Fourier Transform Infra-Red (FTIR), In-Situ and Ex-Situ Mossbauer spectroscopy and $\mathrm{x}$-ray diffraction (XRD).$^{8,9,10,11}$ Although providing partial insight into the charge/discharge mechanism and the nature of the less conductive final discharge product, the exact structural transformations and their relation to the valence state changes and reversibility have not yet been fully elucidated. In this work X-ray Absorption Spectroscopy will be used to gain further information on the final discharge state and the structural transformations that occur during charge/discharge.

The intercalation of $\mathrm{H}^{+}$ions during discharge induces reduction in the Fe valence state and leads to ferrate lattice expansion, $\delta a=a_{c}-a_{d}$ where $a_{c}$ and $a_{d}$ indicate the "effective" lattice constant of the charged and partially discharged state. If this lattice expansion, $\delta \mathrm{a}$, becomes too large to be accommodated, severe instabilities and even phase transitions and phase separation, such as that indicated by eq. 2 :

$$
2 \mathrm{~K}_{2} \mathrm{FeO}_{4}+6 \mathrm{H}^{+}+6 \mathrm{e}^{-} \rightarrow \gamma-\mathrm{Fe}_{2} \mathrm{O}_{3}+4 \mathrm{KOH}+\mathrm{H}_{2} \mathrm{O}
$$

can occur in the crystal structure. ${ }^{12,13}$ The change in crystal structure, such as a change from the tetrahedral ferrate salt structure to the non-conducting $\mathrm{Fe}_{2} \mathrm{O}_{3}$ discharge state, is detrimental to the reversibility of the super iron battery, as conversion back to the ferrate salt structure is apparently not facile both because of the resistive nature of $\mathrm{Fe}_{2} \mathrm{O}_{3}$ and the regional separation into the $\mathrm{Fe}_{2} \mathrm{O}_{3}$ and $\mathrm{KOH}$ phases. 
To prevent the structural changes and accommodate lattice expansion in the superiron salts, two different paths are examined in this work:

1. Accommodating the lattice expansion: Reducing the ferrate salt particle size to the nano-domain facilitates the electron transport rate and diffusion rate of the intercalating $\mathrm{H}^{+}$ ions by providing a shorter diffusion length, and has other advantages. The higher surface area provided by the decrease in particle size increases the contact with the electrolyte interface, it also provides shorter diffusion pathways for the $\mathrm{OH}^{-}$by-product. Further, the lattice expansion is easily accommodated in smaller nanoparticles, thereby relieving the stress that can lead to the unwanted structural and spatial variations in the discharge product. ${ }^{14}$

2. Decreasing the lattice expansion: A second path is to expand the "effective" lattice constant of the ferrate salt in the initial charged state, so that the lattice expansion, $\delta \mathrm{a}$, is reduced. Thus this work focuses on the effect of the cation in $\mathrm{M}_{\mathrm{n}} \mathrm{FeO}_{4}$ on the lattice constant, hydrogen diffusivity, intercalation mechanism, and ultimate conservation of electrode microstructure.

Here, the salts studied are $\mathrm{BaFeO}_{4}$ and $\mathrm{K}_{2} \mathrm{FeO}_{4} \cdot \mathrm{BaFeO}_{4}$ and $\mathrm{K}_{2} \mathrm{FeO}_{4}$ exhibit significantly different electrochemical properties. $\mathrm{K}_{2} \mathrm{FeO}_{4}$ shows a higher solid-state stability ( $0.1 \%$ decomposition per year) and higher intrinsic $3 \mathrm{e}^{-}$capacity compared to pure $\mathrm{BaFeO}_{4}$; however, the rate of charge transfer under similar external load is higher in the latter. The solubility of $\mathrm{K}_{2} \mathrm{FeO}_{4}$ decreases with $\mathrm{KOH}$ concentration, while $\mathrm{BaFeO}_{4}$ is 
considerably less soluble, $2 \times 10^{-4} \mathrm{M}$ in $5 \mathrm{M} \mathrm{KOH}$ containing $\mathrm{Ba}(\mathrm{OH})_{2}$, and nearly insoluble in $\mathrm{Ba}(\mathrm{OH})_{2}{ }^{9}{ }^{9}$ The low $\mathrm{BaFeO}_{4}$ solubility is beneficial, decreasing the possibility of self discharge in the super-iron battery. Although $\mathrm{BaFeO}_{4}$ has a lower theoretical capacity $(313 \mathrm{mAh} / \mathrm{g})$ compared to $\mathrm{K}_{2} \mathrm{FeO}_{4}(406 \mathrm{mAh} / \mathrm{g})$, it has exhibited higher experimental capacity. ${ }^{15}$ Both of these compounds can be synthesized by several routes with high purity as previously described by Licht et. al. in several publications. ${ }^{9,16,17}$

Figure 6.1a illustrates a comparison of electrochemical performance of micron sized $\mathrm{K}_{2} \mathrm{FeO}_{4}$ and $\mathrm{BaFeO}_{4}$, when discharged under a constant load of 1000 ohms, $\mathrm{BaFeO}_{4}$ discharges to more than $90 \%$ of the $3 \mathrm{e}^{-}$columbic efficiency compared to $70 \%$ from $\mathrm{K}_{2} \mathrm{FeO}_{4}$. As illustrated in Figure 1b, the $\mathrm{BaFeO}_{4}$ cathode also exhibits a higher columbic efficiency at higher discharge rates (now with a load of $500 \Omega$ and $\mathrm{J}>10 \mathrm{~mA} / \mathrm{cm}^{2}$ ) providing a higher experimental energy capacity for this compound despite the lower intrinsic charge capacity of $\mathrm{BaFeO}_{4}$ compared with $\mathrm{K}_{2} \mathrm{FeO}_{4}$. Although these two compounds have only a different "spectator" cation with otherwise very similar crystal structure, they exhibit significantly different electrochemical behavior. This suggests that a detailed comparison of their spectroscopic properties might shed new light on the reversibility issue.

In this work, the objective is to investigate the relationship between the structural and valence state properties and the reversibility of this class of novel cathodic materials in aqueous media utilizing X-ray Absorption Spectroscopy (XAS). XAS is an element specific technique; this means that the chemical environment of a specific element in a 

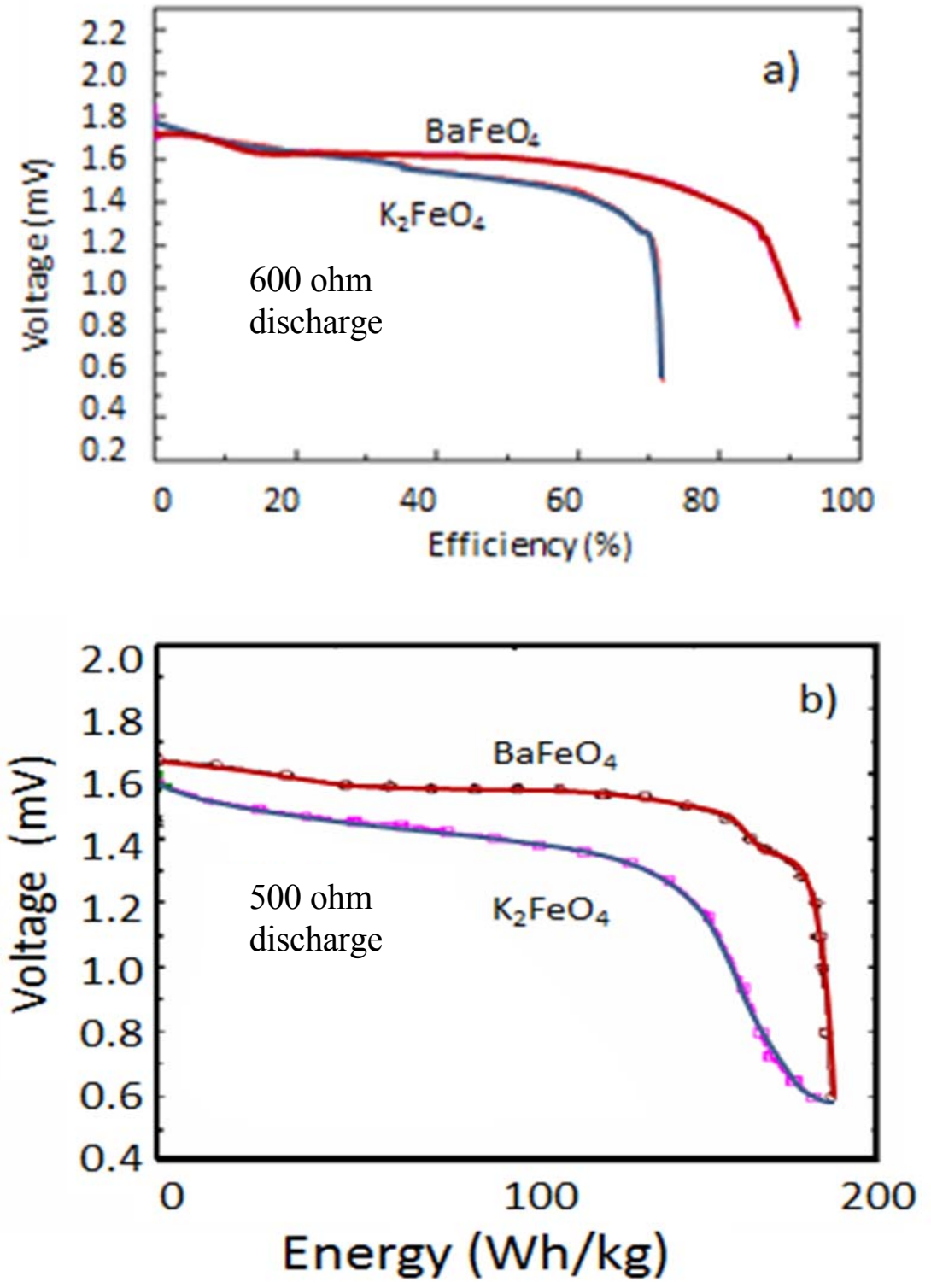

Figure 6.1 a) Comparative discharge of micron-sized $\mathrm{K}_{2} \mathrm{FeO}_{4}$ and $\mathrm{BaFeO}_{4}$ cathodes mixed with $10 \%$ carbon black in a $1 \mathrm{~cm}$ diameter coin cell, $\mathrm{Zn}$ anode and saturated $\mathrm{KOH}$ electrolyte. Discharged under constant load of $1000 \mathrm{ohm} . \mathrm{b}$ ) Energy capacity comparison of $\mathrm{K}_{2} \mathrm{FeO}_{4}$ and $\mathrm{BaFeO}_{4}$ with a $\mathrm{Zn}$ anode. 
complex material such as a cathode can be probed, and this can be performed in situ during charge/discharge. We will take advantage of both the structural information obtained from the EXAFS and the electronic information in the XANES parts of an XAS spectrum utilizing the Fe $\mathrm{K}$ edge. The EXAFS will give us structural information, such as Fe-O bond distances, coordination numbers, and Debye-Waller "disorder" factor, etc., and the XANES provides information on the electronic valence state of Fe. XAS is a short-range order technique; therefore, it can be used to study amorphous materials and small particles.

In our previous paper ${ }^{6}$, it was illustrated that a gentle and low-heat milling procedure, consisting of 6 five minute intervals with $300 \mathrm{rpm}$, improves the electrochemical activity of $\mathrm{K}_{2} \mathrm{FeO}_{4}$. The reduction of particle size from $20-100 \mu \mathrm{m}$ to $\sim 100 \mathrm{~nm}$, as confirmed by SEM imaging, successfully increases the columbic efficiency of the discharge in an alkaline medium from about $20-30 \%$ to about $60 \%-80 \%$ when discharged under a constant load of 600 ohms. Thus the charge/discharge of $\mu \mathrm{m}$ - and $\mathrm{nm}-\mathrm{K}_{2} \mathrm{FeO}_{4}$ will be observed with XAS, along with that of $\mu \mathrm{m}-\mathrm{BaFeO}_{4}$.

\subsection{Experimental}

\subsubsection{Synthesis}

$\mu \mathrm{m}-\mathrm{K}_{2} \mathrm{FeO}_{4}$ was chemically synthesized as previously reported ${ }^{16}$, recrystallized by dissolution in cold $2.67 \mathrm{M} \mathrm{KOH}$, then filtered into cold $12 \mathrm{M} \mathrm{KOH}$ and stirred for 15 min. The product was filtered, and washed in turn with hexane, isopropanol, methanol and ether, and then dried under vacuum for one hour. To prepare $n m-\mathrm{K}_{2} \mathrm{FeO}_{4}$, the chemically synthesized and recrystallized $\mathrm{K}_{2} \mathrm{FeO}_{4}$ was carefully ball milled in a RetschPM100 planetary ball mill with a steel vessel and steel grinding balls. A gentle 
and low-heat ball milling procedure was carefully carried out in short segments, consisting of 6 five minute intervals with $300 \mathrm{rpm}$ as described previously to avoid thermochemical and heat related restructuring and chemical decomposition of the ferrate material. The ball milling procedure resulted in reduction of the particle size from 20 $100 \mu \mathrm{m}$ to $\sim 100 \mathrm{~nm}$ as confirmed by SEM imaging reported previously. ${ }^{6}$

$\mathrm{BaFeO}_{4}$ was synthesized using the chemical synthesis procedure also described previously. ${ }^{16}$ In s brief, the $\mathrm{BaFeO}_{4}$ is obtained directly from the re-crystallized $\mathrm{K}_{2} \mathrm{FeO}_{4}$ utilizing the higher alkaline insolubility of $\mathrm{BaFeO}_{4}$ relative to $\mathrm{K}_{2} \mathrm{FeO}_{4}$. A sample of 20.1 $\mathrm{g}$ of $\mathrm{Ba}(\mathrm{OH})_{2} .8 \mathrm{H}_{2} \mathrm{O}(98 \%$, Sigma Aldrich) was dissolved in $500 \mathrm{ml}$ of doubly deionized water, while argon was bubbled through the solution at $0^{\circ} \mathrm{C}$ to remove $\mathrm{CO}_{2}$. This solution was then filtered through GF/A filter paper $(150 \mathrm{~mm})$. A second solution containing $8 \mathrm{~g}$ of $\mathrm{K}_{2} \mathrm{FeO}_{4}$ in $160 \mathrm{ml}$ of $2 \% \mathrm{KOH}$ was prepared and $\mathrm{CO}_{2}$ was removed with flow of argon at $0^{\circ} \mathrm{C}$ and then filtered through $150 \mathrm{~mm} \mathrm{GF} / \mathrm{A}$ filter paper into the first solution at $0^{\circ} \mathrm{C}$. The resulting mixture was then stirred at $0^{\circ} \mathrm{C}$ for 20 minutes. The $\mathrm{BaFeO}_{4}$ precipitate was filtered and washed with 4 liters of cold distilled water and dried under vacuum for 24 hours.

The $\mu \mathrm{m}-\mathrm{BaFeO}_{4}$ synthesized using this method is relatively pure, but suffers from low -solid state stability. This doesn't permit for ball milling of this salt without thermal decomposition to $\gamma-\mathrm{Fe}_{2} \mathrm{O}_{3}$, thus $\mathrm{nm}-\mathrm{BaFeO}_{4}$ could not be prepared. In the dry state, $\mathrm{BaFeO}_{4}$ was also found to decompose to $\gamma-\mathrm{FeO}_{4}$ in about 3 hours under the x-ray beam. 
Thus extreme care is required, even for micron size particles, to maintain the ferrate structure in the $\mathrm{BaFeO}_{4}$.

\subsubsection{XAS Ex-Situ Measurements}

XAS data were taken at room temperature at the $\mathrm{Fe} \mathrm{K}$ edge at the $\mathrm{X}-3 \mathrm{~B}$ beamline (National Synchrotron Light Source, Brookhaven National Laboratory, Upton, NY) with a Si (111) double crystal monochromator, and collected in fluorescence mode, with the sample placed between two gas ionization detectors and a Canberra 13-element Germanium solid state detector, which was collecting X-rays at a 90 degree angle relative to the incident beam. To account for the drift in the incident beam, a Fe reference foil ( 7 $\mu \mathrm{m}$ thickness) was positioned between the second and third ionization chamber detectors in all measurements.

The super-iron primary cells were prepared in conventional $1.1 \mathrm{~cm}$ diameter A76 button cells. The zinc anode of the commercial button cells was retained, and concentrated $(16 \mathrm{M}) \mathrm{KOH}$ was added as electrolyte. The nm- and $\mu \mathrm{m}-\mathrm{K}_{2} \mathrm{FeO}_{4}$ and $\mu \mathrm{m}$ $\mathrm{BaFeO}_{4}$ were mixed with $10 \%$ carbon and replaced in the cathodic compartment and discharged under a constant load of either 600 or $1000 \Omega$. The cells were discharged to various percentages of the $3 \mathrm{e}^{-}$Columbic efficiency, then opened, and the cathode composite removed, placed between two layers of Kapton tape, and studied with XAS. 


\subsubsection{The $\Delta \mu$-XANES Technique}

The IFEFFIT suite ( Version 1.2.11 IFEFFIT Copyright 2008, Mathew Newville, University of Chicago, http://cars9.uchicago.edu/ifeffit) of programs was used for the $\Delta \mu-$ XANES data analysis including the background subtraction (AUTOBK algorithm) and normalization. This $\Delta \mu$ technique has been described in detail elsewhere. In summary, the raw spectra from different XAS scans were merged together for each single potential. To account for the drift in beam energy over the beam lifetime, these normalized foil spectra were then aligned to one standard foil spectrum. This energy correction is automatically transferred to the sample spectra as well. The success of the $\Delta \mu$ technique is very much dependent on the precision of this energy alignment step. A post-edge normalization procedure (25 to $150 \mathrm{eV}$ above the edge, for $\Delta \mu$ analysis) is then applied to the sample spectra. The $\Delta \mu$ signatures are then obtained by subtracting the $\mu$ for $\gamma-\mathrm{Fe}_{2} \mathrm{O}_{3}$ as reference;

$$
\Delta \mu=\mu(\mathrm{V})-\mu(\mathrm{Ref})
$$

The completely discharged product has been shown previously to be $\gamma-\mathrm{Fe}_{2} \mathrm{O}_{3}$ for nm-

$\mathrm{K}_{2} \mathrm{FeO}_{4}$, and we will show below that this is only nominally true for $\mathrm{BaFeO}_{4}$ but we will use the same reference for all salts.

\subsubsection{EXAFS analysis}


Using the IFEFFIT suite (version 1.2.11 IFEFFIT Copyright 2008, Matthew Newville, University of Chicago, http://cars9.uchicago.edu/ifeffit/) linear combination fitting was conducted on the cathodic composites discharged to various depths after initial data analysis to extract the $\chi(\mathrm{k})$. In this method, the $\chi=\left[\mu(\mathrm{k})-\mu_{0}\right] / \mu_{0}$, of each partially discharged cathode is fit to the $\chi$ for the un-discharged $\mathrm{M}_{\mathrm{n}} \mathrm{FeO}_{4}$ cathode material and the discharge material, $\gamma-\mathrm{Fe}_{2} \mathrm{O}_{3}$ :

$$
\chi(\mathrm{t})=\mathrm{A}^{*} \chi_{\mathrm{MnFeO} 4}+(1-\mathrm{A})^{*} \chi_{\gamma-\mathrm{Fe} 2 \mathrm{O} 3}
$$

where $\mathrm{A}$ is the fraction of the initial ferrate structure $\left(\mathrm{M}_{\mathrm{n}} \mathrm{FeO} \mathrm{O}_{4}\right)$ present in each partially discharged cathode. Eq. (4) assumes that only the two "end" structures are possible, the fully charge and discharged, and this of course is not necessarily the case, as possible partial discharge products are also certainly present. Further, eq. (2) shows that even the final discharge product contains regions of $\mathrm{MOH}_{2-\mathrm{n}}$. Nevertheless, we will show below that the fit of eq. (4) to the experimental $\chi(\mathrm{k})$ obtained from the cathode material at a particular time during the discharge effectively identifies the point where the rather sharp decomposition and/or phase transformation occurs causing the increase in internal resistance, and hence decline in current flow (i.e. the sharp drop in A in eq. (4) appears to strongly correlate with the current drop in a discharge profile such as those shown in Figure 2). 


\subsection{Results and discussion}

\subsubsection{EXAFS Results}

It is important to establish the nature of the fully discharged product. Comparison of the EXAFS spectrum of final discharged cathodes $\chi_{\mathrm{FD}}(\mathrm{k})$, with various iron oxide standards, $\chi_{\text {Stand }}(\mathrm{k})$ reveals that the final discharge product of both $\mathrm{K}_{2} \mathrm{FeO}_{4}$ and $\mathrm{BaFeO}_{4}$ is "mainly" $\gamma$ -

$\mathrm{Fe}_{2} \mathrm{O}_{3}$; that is the residual Res $\left.=\Sigma\left(\chi_{\mathrm{FD}}-\chi_{\mathrm{Stand}}\right)^{2} / \Sigma \chi_{\mathrm{FD}}{ }^{2}\right)$ between the standard $\gamma-\mathrm{Fe}_{2} \mathrm{O}_{3}$ and the final discharge product is 2-3 times less with $\gamma-\mathrm{Fe}_{2} \mathrm{O}_{3}$ (at least for $\mathrm{K}_{2} \mathrm{FeO}_{4}$ ) than that with other standards as shown in Figure 6.2. In the expression Res, $\chi(\mathrm{k})$ was summed over points in the range $3<\mathrm{k}<10$ and the $\chi_{\mathrm{FD}}$ and $\chi_{\text {Stand }}$ are the EXAFS $\chi$ functions of the fully or 8 hour discharged cathode (as indicated in Figure8) and the standard that is being examined. These results suggest that for $\mathrm{nm}-\mathrm{K}_{2} \mathrm{FeO} 4$, the discharge product is indeed more like $\gamma-\mathrm{Fe}_{2} \mathrm{O}_{3}$, but for the $\mathrm{BaFeO}_{4}$, it may be partially hydrated at least initially, since the residual with $\gamma-\mathrm{Fe}_{2} \mathrm{O}_{3}$ is only slightly less than for the hydrated oxides after just 8 hours, but then dehydrates some after longer periods.

The change in crystal structure during discharge is from that of a ferrate, to a partially discharged ferrate but still in its ferrate ionic lattice, and ultimately to full lattice rearrangement with regions of more extended Fe-O-Fe covalent bonding existing in $\gamma$ $\mathrm{Fe}_{2} \mathrm{O}_{3}$ oxide along with regions of $\mathrm{KOH}$, as suggested by eq. (2). The sizes of the alkaline hydroxides and the iron oxide regions are not known, but this is certainly one reason why the comparison of the $\chi(\mathrm{k})$ 's in Figure2 cannot give significantly lower residuals; we are comparing the discharge product (interspersed iron oxide $+\mathrm{K}$ or Ba hydroxide regions of 


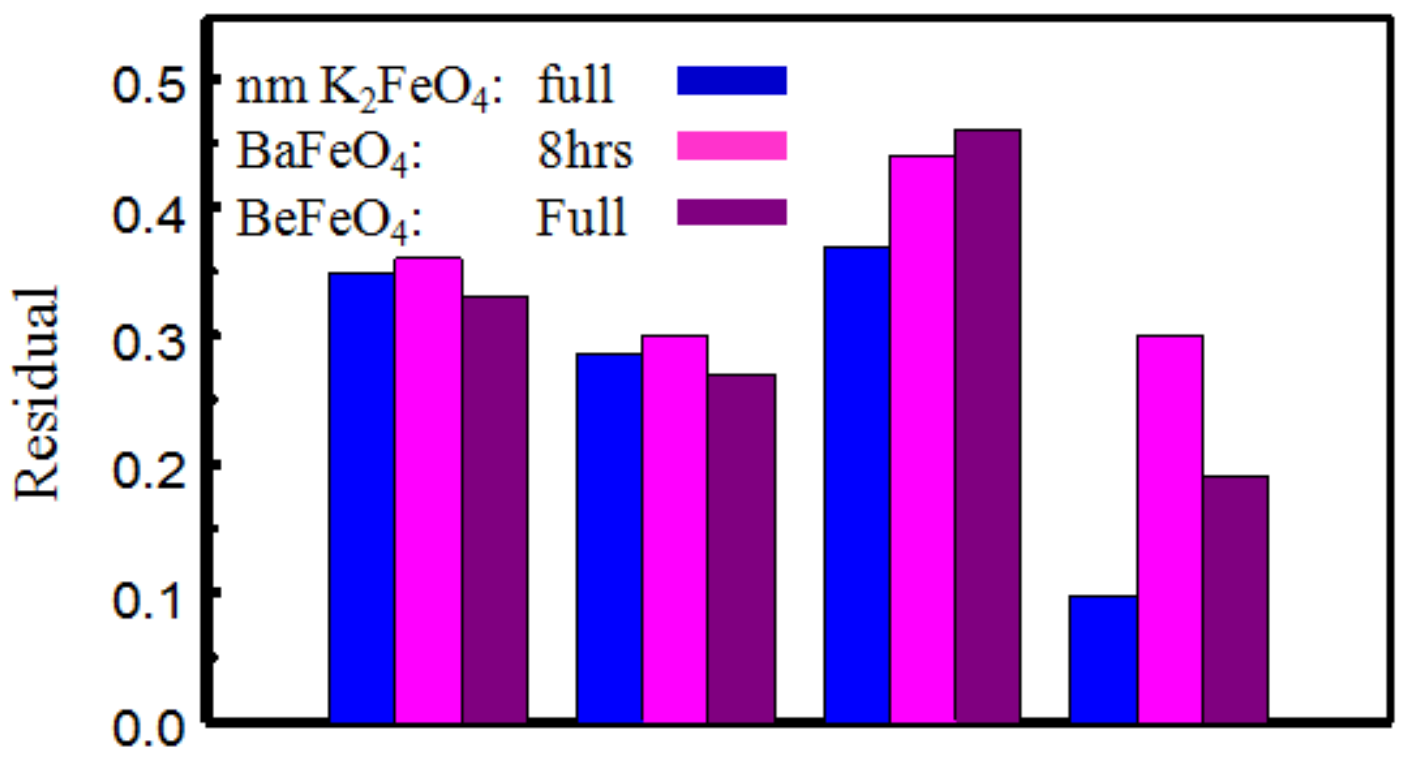

Figure 6. 2. Residuals defined as described in text between the full discharge product for nm- $\mathrm{K}_{2} \mathrm{FeO}_{4}$ and that after 8 hours and full discharge of $\mu \mathrm{m}-\mathrm{BaFeO}_{4}$ compared to the standards indicated. 
Unknown size) with a pure iron oxide standard. In contrast with that of previously reported FT-IR studies and in-situ Mossbauer spectroscopy ${ }^{8}$ on $\mathrm{BaFeO}_{4}$, which could not point to a specific ferric oxide or hydroxide as the main discharge product, the EXAFS features for the $\mathrm{BaFeO}_{4}$ discharge product do show slightly better agreement with the $\gamma$ $\mathrm{Fe}_{2} \mathrm{O}_{3}$ form of the oxide, similar to that clearly found for $\mathrm{K}_{2} \mathrm{FeO}_{4}$.

However, conclusive evidence for the discharge produce in the case of $\mathrm{BaFeO}_{4}$ is not indicated here either, because the residual is not sufficiently reduced compared with the other iron oxyhydroxides or oxides.

To study the structural and morphological properties of the cathode through the course of the discharge, linear combination EXAFS fitting with the procedure described above, was conducted on each partially discharged cathode (for $\mu \mathrm{m}$ - and nm- $\mathrm{K}_{2} \mathrm{FeO}_{4}$ and $\mathrm{BaFeO}_{4}$ ) in the $\mathrm{k}$ range of 3 to 12 . Example fits for two of the partially discharged nm$\mathrm{K}_{2} \mathrm{FeO}_{4}$ cathodes are shown in Figure 6.3, as well as the constituent components and their contribution to the final fit. As the discharge progresses from 3 hours to 16 hours, the composition of the cathode changes from $95 \%$ to only $17 \%$ undischarged $\mathrm{K}_{2} \mathrm{FeO}_{4}$. The quality of the fits are acceptable for the undischarged and partially discharged cathodic composites, however the quality of the fits decreases for the deeply discharged cathodes. Fit R-factors increase from 0.000744 for a 3 hours discharged cathode (when comparing mostly undischarged product with completely undischarged material) to 0.002753 for 16 hours of discharge and to 0.171459 for a completely discharged nano- $\mathrm{K}_{2} \mathrm{FeO}_{4}$ cathode as shown in Figure 6.3. We emphasize again that the full $3 \mathrm{e}^{-}$discharge radically disrupts the 195 

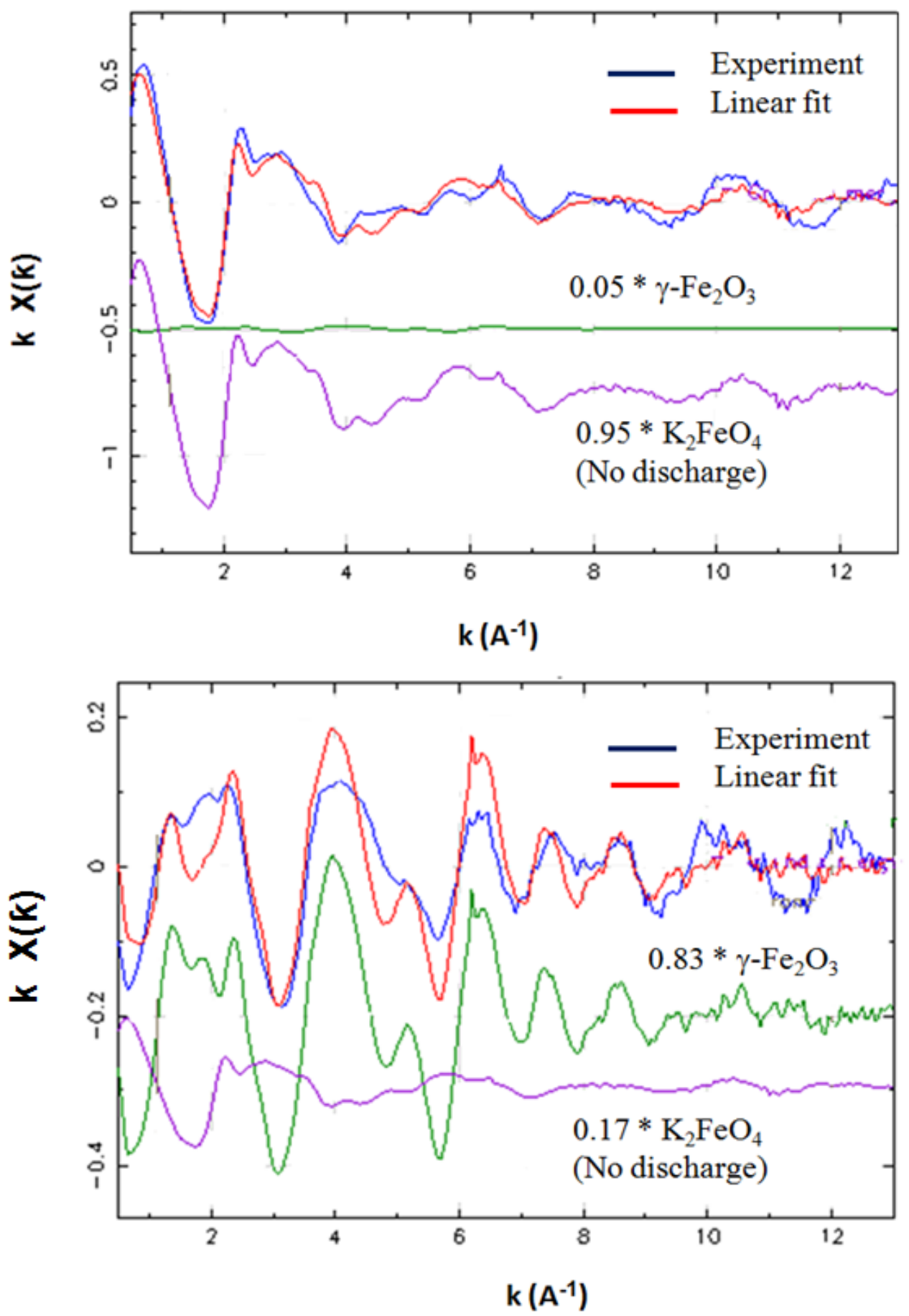

Figure 6.3. Linear EXAFS component fits for two $\mathrm{nm}-\mathrm{K}_{2} \mathrm{FeO}_{4}$ discharged cathodes: a) cathode discharged for 3 hours. b) cathode discharged for 16 hours. 
lattice and forms a two phase (metal hydroxide and iron oxide) material that is structurally non-reversible; i.e. it cannot return to the initial charged Fe(VI) ionic ferrate state. Nevertheless, the linear combination fit provides a tool to quantify the level of discharge, or discharge time, when this structural change occurs in the cathode.

\subsection{2 $\Delta \mu$ Results}

Identifying the nature of the main discharge product using the EXAFS procedure above to follow the structural change is useful, but it also useful to follow the $\mathrm{Fe}$ oxidation state in the cathode material with discharge time. This can be done with the XANES data. Figure 6.4 shows the significant difference between the XANES spectra for the $\mathrm{BaFeO}_{4}$ (or $\mathrm{K}_{2} \mathrm{FeO}_{4}$ not shown) and the $\gamma-\mathrm{Fe}_{2} \mathrm{O}_{3}$. The $\mathrm{BaFeO}_{4}$ has the strong preedge peak from the non-dipole allowed $1 \mathrm{~s}$ to $3 \mathrm{~d}$ transition, and the higher energy "whiteline resulting from the $1 \mathrm{~s}$ to np excitation. This whiteline moves up in energy with higher oxidation state of the iron as expected. The $1 \mathrm{~s}$ to $3 \mathrm{~d}$ transition is only visible in the XAS spectrum because of $4 p$ and $3 d$ orbital mixing, and this can strongly occur only in a non-octahedral environment, such as the tetrahedral environment around the $\mathrm{Fe}$ atoms in the $\mathrm{FeO}_{4}$ structure present in the ferrates. The $1 \mathrm{~s}$ to $3 \mathrm{p}$ peak is mostly absent in $\gamma-\mathrm{Fe}_{2} \mathrm{O}_{3}$, due to the $\mathrm{FeO}_{6}$ near octahedral environment existing in $\gamma-\mathrm{Fe}_{2} \mathrm{O}_{3}$, which does not allow p-d orbital mixing. ${ }^{20}$ The intensity of the pre-edge feature decreases upon reduction in part due to the increased electron population of the $3 \mathrm{~d}$ state, namely from approximately $\mathrm{d}^{2}$ in $\mathrm{Fe}^{6+}$ to $\mathrm{d}^{5}$ in $\mathrm{Fe}^{3+}$ (i.e. less empty $\mathrm{d}$ states exist for the photoelectron to be excited into in the XAS process); however, this alone cannot account for the factor 


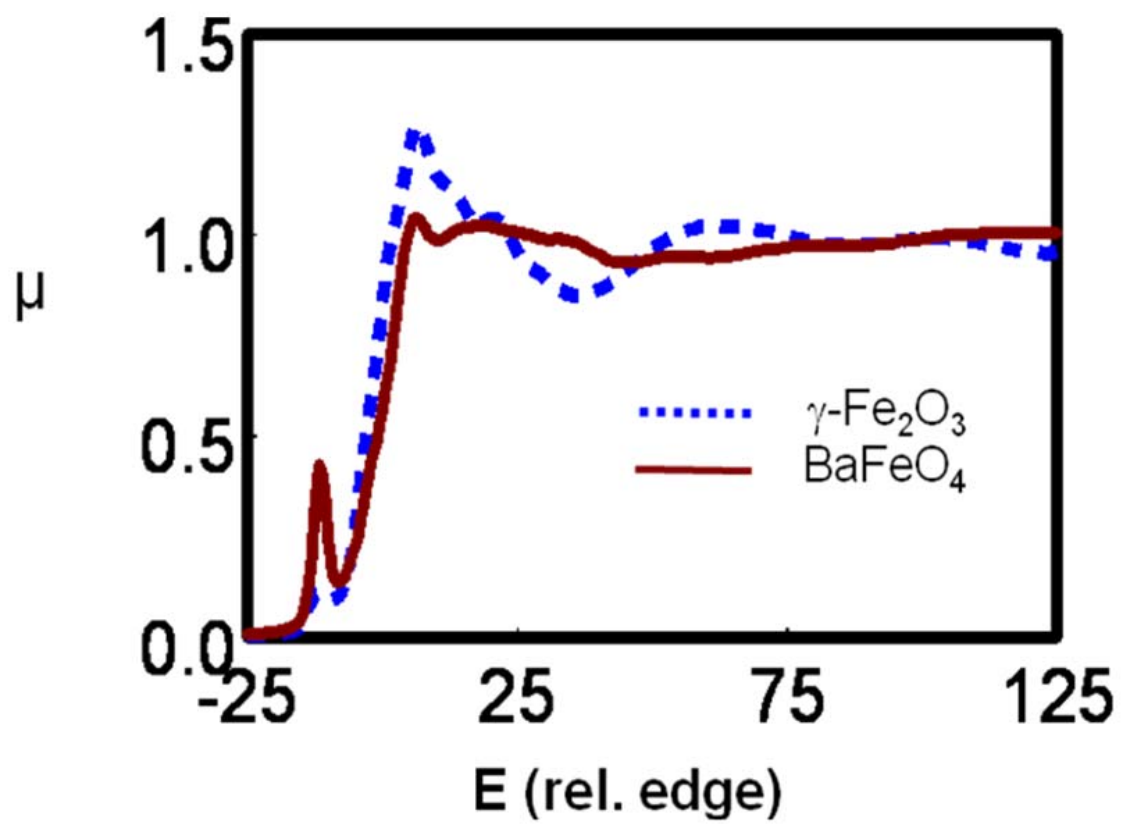

Figure 6.4. XAS data, $\mu(\mathrm{E})$ for $\mathrm{BaFeO} 4$ and $\gamma-\mathrm{Fe}_{2} \mathrm{O}_{3}$ as standard, where the data for $\mathrm{BaFeO}_{4}$ is for the undischarged micron sized cathode. 


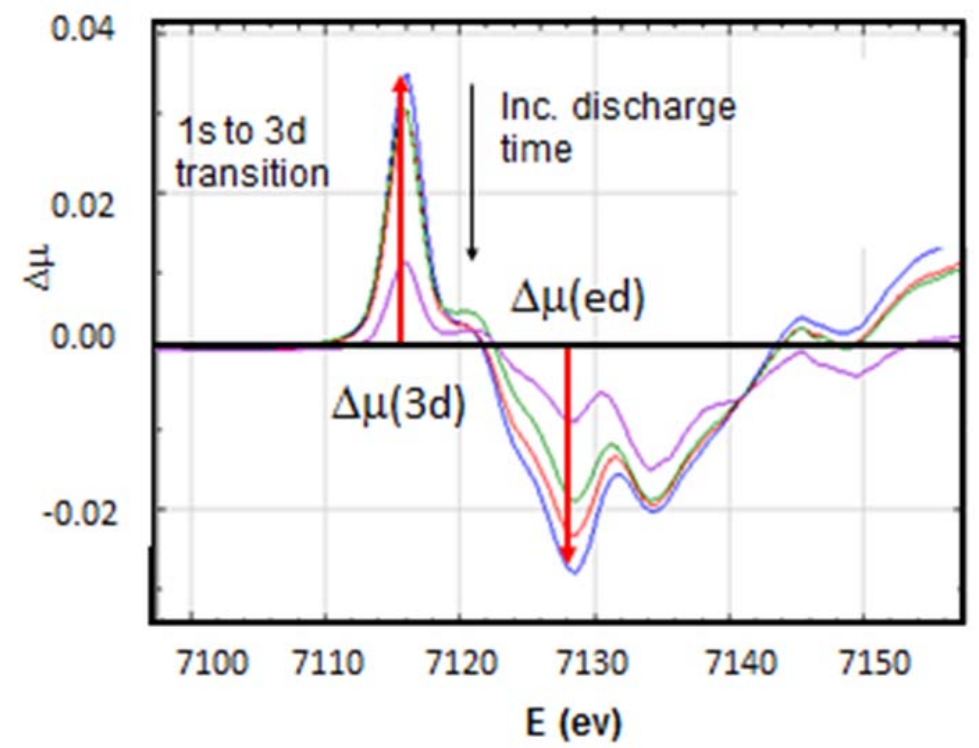

Figure 6.5. $\Delta \mu$ results for $\mathrm{nm}_{-} \mathrm{K}_{2} \mathrm{FeO}_{4}$ at 3,5,8, and $16 \mathrm{hrs}$ discharge time under $600 \Omega$ load. The magnitudes of the $\Delta \mu(3 \mathrm{~d})$ and $\Delta \mu(\mathrm{ed})$ are indicated by the red arrow. 
of 4-6 decrease in the pre-edge peak with reduction of the iron, as the number of vacant $d$ slots only decreases from about 8 to 5 , a factor of 1.6. Thus the decrease in the pre-edge feature apparently also comes from a symmetry change about the Fe atom, namely from a tetrahedral to a more octahedral environment.

This change in coordination and hence average symmetry about the Fe atoms can be accounted for via two possible mechanisms: I) assuming that discharge of the ferrate cathode involves intercalation of a proton through the electrolyte and an electron via the circuit along with some atom rearrangement, and II) intercalation of a water molecule and an electron, $\mathrm{H}_{2} \mathrm{O}+\mathrm{e}^{-}$, so that reduction involves $\mathrm{M}_{\mathrm{n}} \mathrm{FeO}_{4}$ going to $\mathrm{M}_{\mathrm{n}} \mathrm{FeO}_{4}\left(\mathrm{H}_{2} \mathrm{O}\right)_{\mathrm{n}}{ }^{\mathrm{n}-}$. In this mechanism, the separation of the $\mathrm{OH}^{-}$assumed in eq. 1 does not occur, rather the $\mathrm{OH}^{-}$group remains with the Fe atom. In mechanism $\mathrm{I}$, addition of $\mathrm{H}$ atoms $\left(\mathrm{H}^{+}+\mathrm{e}^{-}\right.$with $\mathrm{OH}$ - remaining behind) may result in the formation of a "cross-link" between neighboring ferrate anions with formation of a dimer-like species:

$$
2 \mathrm{M}_{\mathrm{n}} \mathrm{FeO}_{4}+2 \mathrm{H}^{+} \rightarrow \mathrm{M}_{\mathrm{n}}\left[(\mathrm{OH}) \mathrm{O}_{2} \mathrm{Fe}<\mathrm{O}_{\mathrm{O}}>\mathrm{FeO}_{2}(\mathrm{OH})\right] \mathrm{M}_{\mathrm{n}}
$$

In $\gamma-\mathrm{Fe}_{2} \mathrm{O}_{3}$, each $\mathrm{O}$ atom is coordinated to $4 \mathrm{Fe}$ atoms with tetrahedral symmetry around the $\mathrm{O}$ atoms. This is in contrast to $\mathrm{K}_{2} \mathrm{FeO}_{4}$, where the $\mathrm{O}$ atoms are coordinated to just one $\mathrm{Fe}$ atom. Thus the $\mathrm{Fe}$ coordination increases from 4 (tetrahedral) to 6 (octahedral), but the $\mathrm{O}$ coordination increases even more from 1 to 4 upon reduction. The dimer-like species (and trimer, tetramer, to ultimately polymer) with $\mathrm{O}$ coordination increasing from 1 to 2 serve as a precursor to the fuller lattice rearrangement and 
separation into hydroxide and iron oxide region mapped by the EXAFS fitting (with O coordination in the iron oxide equal to 4). It is likely that $\mathrm{O}$ coordination much great than 2 or 3 cannot occur unless the full lattice rearrangement occurs. After formation of the polymer-like precursors, the coordination around the Fe atoms has increased to 5 or 6 , and this will result in the loss of some $3 \mathrm{~d}$ intensity, as the symmetry changes from tetrahedral to more octahedral-like symmetry.

In mechanism II (water intercalation), the Fe coordination can change from 4 with tetrahedral symmetry, $\left(\mathrm{FeO}_{4}\right)^{2-}$, in the ferrate, to 6 in $\mathrm{FeO}_{2}(\mathrm{OH})_{4}{ }^{5-}$ with near octahedral symmetry, but without significant increase in the $\mathrm{O}$ coordination. In this way a hydrated ferric oxide is initially formed, which ultimately converts to iron(III) oxide upon dehydration and separation into metal hydroxide and iron oxide regions.

In the two mechanisms described above, the oxidation state can change with or without increase of the Fe and $\mathrm{O}$ coordination's and resultant symmetry, even if the total crystal structure has not yet changed. The Fe oxidation state can be tracked by following the shift in the Fe k-edge, as it is reflected in the magnitude of the peak in the $\Delta \mu$ signal at photon energy 7124-7129, or just $2-3 \mathrm{eV}$ from the edge around $7126.5 \mathrm{eV}$. We will show below that $\mid \Delta \mu($ ed $) \mid$ does vary nearly linearly with time during the discharge and changes very differently than the magnitude of the pre-edge peak, $|\Delta \mu(3 d)|$, because the latter involves the local geometric symmetry around the Fe, while $\Delta \mu(\mathrm{ed})$ involves only the electronic oxidation state. By following both as a function of time, we can gain 
significant insight into how the reduction and resultant change in structure is taking place.

\subsubsection{Comparison of $\mathrm{nm}-\mathrm{vs} \mu \mathrm{m}-\mathrm{K}_{2} \mathrm{FeO}_{4}$}

Figure 6.6 shows the $\Delta \mu$ amplitudes, $|\Delta \mu(\mathrm{ed})|$ and $|\Delta \mu(3 \mathrm{~d})|$, which track the $\mathrm{Fe}$ oxidation state and change of symmetry about the $\mathrm{Fe}$ atom, respectively, and the A(EXAFS) coefficient from the EXAFS linear fit, which tracks the fraction of ferrate still remaining in the cathode with discharge time. We report the discharge profiles in Figure 6.1 with discharge using a $1000 \Omega$ load, but Fig 6.6 shows XAS data with the smaller $600 \Omega$ load. The A(EXAFS) coefficient shows that the rearrangement of the crystal structure from mostly ionic ferrate-like to $\gamma-\mathrm{Fe}_{2} \mathrm{O}_{3}$ is rather sharp on a $20 \mathrm{hr}$ time scale, and occurs after 9 hours into the discharge, for both the nm- and $\mu \mathrm{m}-\mathrm{K}_{2} \mathrm{FeO}_{4}$ particles (i.e. ground and unground). However note that this transition is much sharper for the much smaller nm particles as might be expected.

Figure 6.6 shows that $|\Delta \mu(\mathrm{ed})|$ varies linearly with time from 0 to about 9 hours, and then quite abruptly changes slope above 9 hours; i.e. right after the crystal structure rearrangement. The slope of the decline in $\mid \Delta \mu($ ed) $\mid$, i.e., $\mathrm{d}|\Delta \mu(\mathrm{ed})| / \mathrm{dt}$, should track with the current, and the current sharply decreases at 9 hours when the rearrangement occurs, because at this point the resistivity of the cathode material $\left(\mathrm{R}_{\mathrm{int}}\right)$ sharply increases; and I $=\mathrm{V} /\left(\mathrm{R}_{\text {int }}+\mathrm{R}_{\text {load }}\right)$. Note that the slope, $\mathrm{d}|\Delta \mu(\mathrm{ed})| / \mathrm{dt}$, (light blue line for the $\mathrm{nm}$ particles) is larger for the nm particles than for the $\mu \mathrm{m}$ particles, consistent with the greater conductivity of the nm material. The crystal rearrangement happens to occur around the 


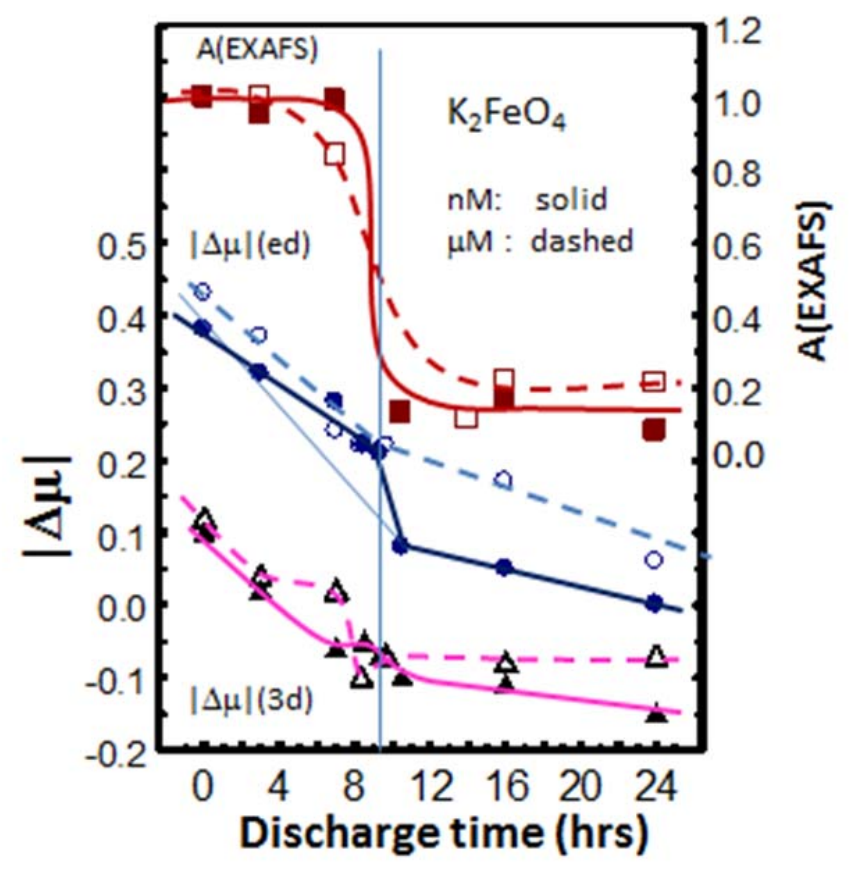

Figure 6.6. Plot of the indicated $\Delta \mu$ amplitudes (at the edge and pre-edge peaks) and $\mathrm{A}$ (EXAFS) for the nm- and $\mu \mathrm{m}-\mathrm{K}_{2} \mathrm{FeO}_{4}$ as a function of discharge time. The light solid blue line on the $\mid \Delta \mu\left(\right.$ ed)| plot suggest how it would change if just $\mathrm{H}^{+}$was intercalated. Measurements were taken on 50 mAh cells with cathodes composed of $10 \%$ carbon black, $90 \%$ of $\mathrm{K}_{2} \mathrm{FeO} 4$ and $16 \mathrm{M} \mathrm{KOH}$ electrolyte and discharged under constant load of 600 ohms. Vertical line drawn at rearrangement. 
same discharge time, but because of the larger current flow in the nm material, the discharge capacity prior to rearrangement is much greater for the nm particles, consistent with the current versus voltage discharge curves reported previously. ${ }^{6}$ The capacity can be predicted from Figure 6.6 by the total drop in $\mid \Delta \mu($ ed) $\mid$ from 0.45 to 0.1 , a $75 \%$ drop for the $\mathrm{nm}$ material, versus a $50 \%$ drop $(0.45$ to 0.23$)$ for the $\mu \mathrm{m}$ material. These are consistent with the capacities reported for these materials previously under a $600 \Omega$ load (these capacities are somewhat larger under a $1000 \Omega$ load as shown in Figure1a). Note that the $\mathrm{d} \mid \Delta \mu($ ed) $) / \mathrm{dt}$ slope above 9 hours is lower in the nm material, consistent with the greater conversion to the resistive $\gamma-\mathrm{Fe}_{2} \mathrm{O}_{3}$ in the $\mathrm{nm}$ material as shown by the A(EXAFS) magnitude; i.e., the lattice conversion is more complete in the nm materials, but occurs at a larger capacity.

Figure 6.6 shows a very large $\mathrm{d} \mid \Delta \mu(\mathrm{ed}) / \mathrm{dt}$ slope in a narrow region around 9 hours in the nm material. This can best be understood in the context of the behavior in $|\Delta \mu(3 \mathrm{~d})|$. Recall that $|\Delta \mu(3 \mathrm{~d})|$ tracks the local symmetry change around the Fe atom. In the $\mu \mathrm{m}$ material, the decrease in $|\Delta \mu(3 \mathrm{~d})|$ with time is strongly delayed between 3-8 hours, then strongly increases between 8 and 9 hours. This suggests that that $\mathrm{H}^{+}$is primarily intercalated between 3-8 hours, i.e. only Fe oxidation state changes but without change in Fe-O coordination and symmetry. This difference in the drop between $\Delta \mu($ ed) and $\Delta \mathrm{M}(3 \mathrm{~d})$ can be regarded as an indication of "stress" build-up, as the lattice wants to rearrange and allow the symmetry around the Fe to change but can't under the constraints of the lattice. The stress is relieved only when the Fe symmetry is allowed to change 
during the lattice rearrangement. Thus the changes in $|\Delta \mu(3 \mathrm{~d})|$ in the $\mu \mathrm{m}$ material occur as expected, i.e., as $\mathrm{H}^{+}$intercalation proceeds, a lag in $|\Delta \mu(3 \mathrm{~d})|$ drop occurs prior to the lattice rearrangement, followed by a steep drop during the rearrangement.

The nm material exhibits a significantly different behavior. Except for a slight "bump" at $9 \mathrm{hrs}, \Delta \mu(3 \mathrm{~d})$ decreases rather uniformly with time, and now $\Delta \mu(\mathrm{ed})$ shows a lag and then sudden drop at 9 hours. How do we account for this significantly different behavior? These results are consistent with much more $\mathrm{H}_{2} \mathrm{O}$ intercalation (i.e., mechanism II when the $\mathrm{OH}^{-}$stays with the $\mathrm{Fe}$ ), and therefore increase in $\mathrm{Fe}-\mathrm{O}$ coordination with gradual change in symmetry from tetrahedral to octahedral symmetry. This intercalation of $\mathrm{H}_{2} \mathrm{O}$ then apparently allows the incoming electrons to be distributed more evenly about the $\mathrm{FeO}_{4}\left(\mathrm{H}_{2} \mathrm{O}\right)_{\mathrm{n}}$ [or $\left.\mathrm{FeO}_{4-\mathrm{n}}(\mathrm{OH})_{2 \mathrm{n}}\right]$ environment, thus delaying the reduction in the Fe valence state. At 8-9 hours the lattice rearrangement separates the water and $\mathrm{KOH}$ from the $\gamma-\mathrm{Fe}_{2} \mathrm{O}_{3}$, so that the $\mathrm{Fe}$ oxidation state now falls quickly as the $\mathrm{OH}^{-}$separates from the $\mathrm{Fe}$ forcing the electrons onto the $\mathrm{Fe}$ atoms.

These results reveal the most important reason why the ground $\mathrm{nm}$ material has increased capacity compared to the $\mu \mathrm{m}$ material. Not only is the conductivity (to electrons) increased, but apparently more important, the greater $\mathrm{K}_{2} \mathrm{FeO}_{4}$-electrolyte interface area in the nm material increases the ability of the larger $\mathrm{H}_{2} \mathrm{O}$ species to intercalate at, and near, the surface of the smaller particles, and thereby decrease the reduction of the Fe valence by distributing some of the electrons onto the $\mathrm{OH}$ or waters. 
The reduction of the Fe is the apparent "stress" which induces the unwanted lattice rearrangement, and intercalation of $\mathrm{H}_{2} \mathrm{O}$, rather than $\mathrm{H}$, enables reduction in this stress by distribution of the electrons more about the intercalated $\mathrm{H}_{2} \mathrm{O}$ coordinating with the $\mathrm{Fe}$. Thus, as might be expected $\mathrm{H}_{2} \mathrm{O}$ intercalation is as important, or more so, than the electron conductivity for increasing capacity.

\subsubsection{Comparison of $\mathrm{BaFeO}_{4}$ with $\mathrm{K}_{2} \mathrm{FeO}_{4}$}

Figure 6.7 shows results for $\mu \mathrm{m}-\mathrm{BaFeO}_{4}$, which are quite different from those for $\mathrm{K}_{2} \mathrm{FeO}_{4}$. In $\mathrm{BaFeO}_{4}, \mid \Delta \mu($ ed $) \mid$ tracks remarkably similarly with $|\Delta \mu(3 \mathrm{~d})|$ and both decrease uniformly with time; i.e. there is no apparent lag in either. Note that $\mid \Delta \mu($ ed) $\mid$ varies linearly again, but with much larger slope before the lattice rearrangement than after the lattice rearrangement. Here we note another significant difference with the $\mathrm{K}_{2} \mathrm{FeO}_{4}$ results. The slope prior to 4 hours is much larger for $\mathrm{BaFeO}_{4}$ compared with even the nm $\mathrm{K}_{2} \mathrm{FeO}_{4}$, consistent with the greater charge transfer rate exhibited by $\mathrm{BaFeO}_{4}$ as discussed in the Introduction. This also accounts for the rearrangement occurring already after 3.8 hrs compared with around $9 \mathrm{hrs}$ for the $\mathrm{K}_{2} \mathrm{FeO}_{4}$. However, even at times longer than 3.8 hrs, i.e., after the lattice rearrangement, the slope is still relatively large at about $0.025 / \mathrm{hr}$, comparable to what it is for $\mathrm{K}_{2} \mathrm{FeO}_{4}$ before the rearrangement. Note that the capacity at 3.8 hours is only about $50 \%$, so that indeed the larger capacity (about $70-80 \%$ ) exhibited in $\mathrm{CV}$ plots for $\mathrm{BaFeO}_{4}$ with $600 \Omega$ load apparently extends beyond the initial lattice rearrangement visible from the EXAFS fit. 


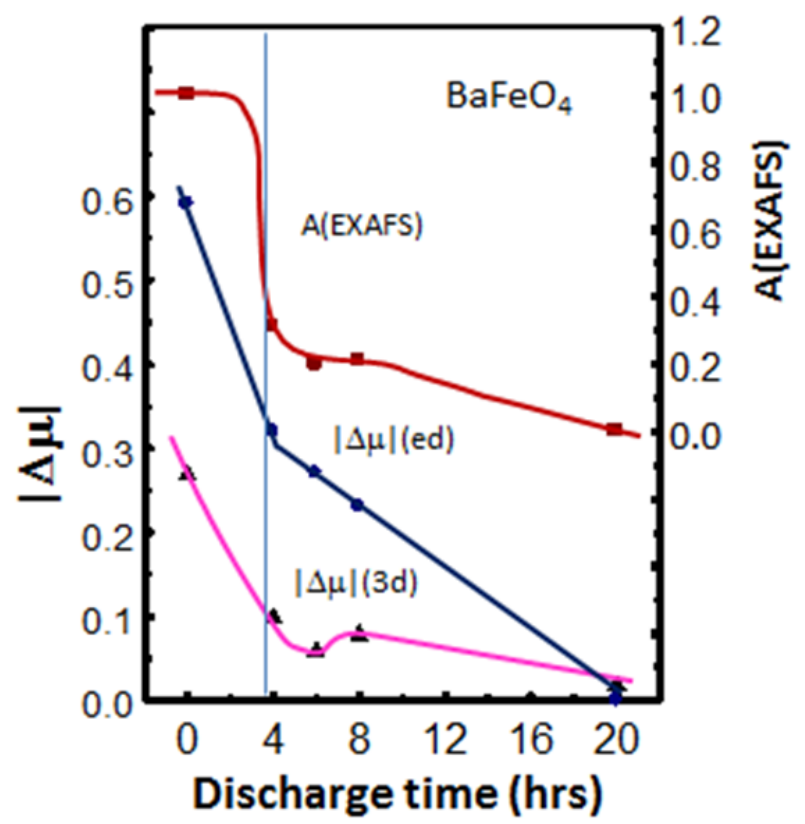

Figure 6.7. Plot of $\Delta \mu$ at the $3 \mathrm{~d}$ pre-edge and edge and A(EXAFS) vs. discharge time for $\mathrm{BaFeO}_{4}$. Vertical line drawn at rearrangement. 
What can account for the high current in $\mathrm{BaFeO}_{4}$ even after the apparent lattice rearrangement at $3.8 \mathrm{hrs}$ as indicated by the EXAFS? We suggest a possible two step lattice rearrangement process or more gradual slow change over to $\gamma-\mathrm{Fe}_{2} \mathrm{O}_{3}$ if at all, beyond 10 hours. Unfortunately, we do not have any points between 8 hours and the nominal full discharge at 20 hours in Figure 6.7, and the difficulties with acquiring synchrotron time, etc. do not allow us readily return and get more points in this region, but other data are consistent with a two-step rearrangement process. Figure 6.1.b shows a small initial drop in $\mathrm{V}$ around $160 \mathrm{Wh} / \mathrm{kg}$ followed by the major drop somewhat later. Further, Figure 2 indicates that the discharge products at 8 hours and at full discharge are different (at least the Res is very different in these two cases). Clearly the rearrangement into separate $\mathrm{Ba}(\mathrm{OH})_{2}$ and $\gamma-\mathrm{Fe}_{2} \mathrm{O}_{3}$ regions does not occur as quickly in time and as fully as the separation into the $\mathrm{KOH}$ and $\gamma-\mathrm{Fe}_{2} \mathrm{O}_{3}$ regions evident in the $\mathrm{nm}-\mathrm{K}_{2} \mathrm{FeO}_{4}$ material. This is perhaps not surprising considering the bigger $\mu \mathrm{m}$-sized $\mathrm{BaFeO}_{4}$ particle; the rearrangement also occurs slower in the $\mu \mathrm{m}-\mathrm{K}_{2} \mathrm{FeO}_{4}$ material, beginning already after 4 hrs and extending to $16 \mathrm{hrs}$. But even after $20 \mathrm{hrs}$ (nominally full discharge), the discharge product does not reflect full $\gamma-\mathrm{Fe}_{2} \mathrm{O}_{3}$ conversion as suggested by the larger Res. in Figure 6.2., but rather a more hydrated structure with the $\mathrm{OH}$ groups still present near the Fe atoms.

In all cases the XAS data simply reflect the "average" conditions existing in the small volume (i.e. cross-sectional area times path length) sampled by the transmitted light beam. In the $\mathrm{nm}-\mathrm{K}_{2} \mathrm{FeO}_{4}$ material, some particles could completely undergo the lattice 
rearrangement into separated regions of $\gamma-\mathrm{Fe}_{2} \mathrm{O}_{3}$ and metal hydroxide, while other particles remain partially or even fully charged. In the $\mu \mathrm{m}$ sized particles $\left(\mathrm{BaFeO}_{4}\right.$ or $\mathrm{K}_{2} \mathrm{FeO}_{4}$ ), it is more probable that individual particles have both regions existing at the same time, i.e., more fully discharged lattice rearranged regions and still charged regions in the same $\mu \mathrm{m}$-sized particle, arranged in some unknown core-shell or mosaic like structure similar to that evident in other battery materials. ${ }^{28,29,30}$ This would explain the slower lattice change-over with time evident in the A(EXAFS) seen with the $\mu \mathrm{m}$-sized particles. But in all cases, the drop-off in A(EXAFS) occurs simultaneously with a significant change in slope of the $\Delta \mu(\mathrm{ed})$ line, and these two data indicators come from the same XAS scan (i.e. exactly the small volume sampled by the light beam). Thus at least on the scale of the XAS sampled volume (millimeters), the discharge appears to be occurring rather uniformly and the EXAFS data is indeed representative of the final discharge product.

The above results strongly suggest that the final discharge product with $\mathrm{BaFeO}_{4}$ is not the same as in $\mathrm{K}_{2} \mathrm{FeO}_{4}$, i.e., the separation into $\mathrm{KOH}$ and $\gamma-\mathrm{Fe}_{2} \mathrm{O}_{3}$ appears to be much more complete and the separated regions are spatially larger in the case of $\mathrm{K}_{2} \mathrm{FeO}_{4}$ than for $\mathrm{BaFeO}_{4}$. This less complete separation makes the discharge product much more conductive to both electrons and protons, so that the discharge can continue even after the lattice rearrangement indicated by the EXAFS fitting. Thus both the charged and discharged end materials are more conductive in $\mathrm{BaFeO}_{4}$ than in $\mathrm{K}_{2} \mathrm{FeO}_{4}$. 


\subsubsection{Intercalation Channels and Lattice Expansion}

At higher discharge rate, both $\mathrm{nm}-\mathrm{K}_{2} \mathrm{FeO}_{4}$ and $\mu \mathrm{m} \mathrm{BaFeO}_{4}$ exhibit higher capacities than $\mu \mathrm{m}-\mathrm{K}_{2} \mathrm{FeO}_{4}$. However, the mechanism by which the intercalation tolerance is increased differs for these two cases.

Others ${ }^{21,23,24,25}$ have previously observed that nm-size particles can enable electrode reactions that do not occur in the $\mu \mathrm{m}$-size domain. The rate and ability of intercalant diffusion (here $\mathrm{H}_{2} \mathrm{O}$ ) is increased in nano-particles, due to the shorter diffusion length and increased electrode/electrolyte interface (SEI) as compared to larger particles. Here the decreased diffusion length required for the $\mathrm{H}_{2} \mathrm{O}$ in the nanometer particles decreases the Fe reduction as noted above, and therefore better "accommodates" the stress induced in the lattice.

XRD measurements ${ }^{26}$ indicate that one dimensional intercalation channels exist in the ' $\mathrm{a}$ ' and ' $\mathrm{b}$ ' directions of the $\mathrm{K}_{2} \mathrm{FeO}_{4}$ unit cell with a radius of $0.93 \mathrm{~A}$. The sharpening of the diffraction peaks observed in the (002), (004) and (006) planes along with the broadening of other features indicates that the intercalants move along these existing one dimensional channels. Figure 6.8 shows the unit cell for $\mathrm{K}_{2} \mathrm{FeO}_{4}$ as viewed from the ' $a$ ' and ' $b$ ' directions. The channels are situated along the Fe-O bonds, and this is where the diffusion of intercalating ions occurs. Diffusion of $\mathrm{H}^{+}$along these channels leads to their association with the oxygens of the ferrate anion. The stability of the initial structure (which is related directly to the stability of the intermediate species) and the 


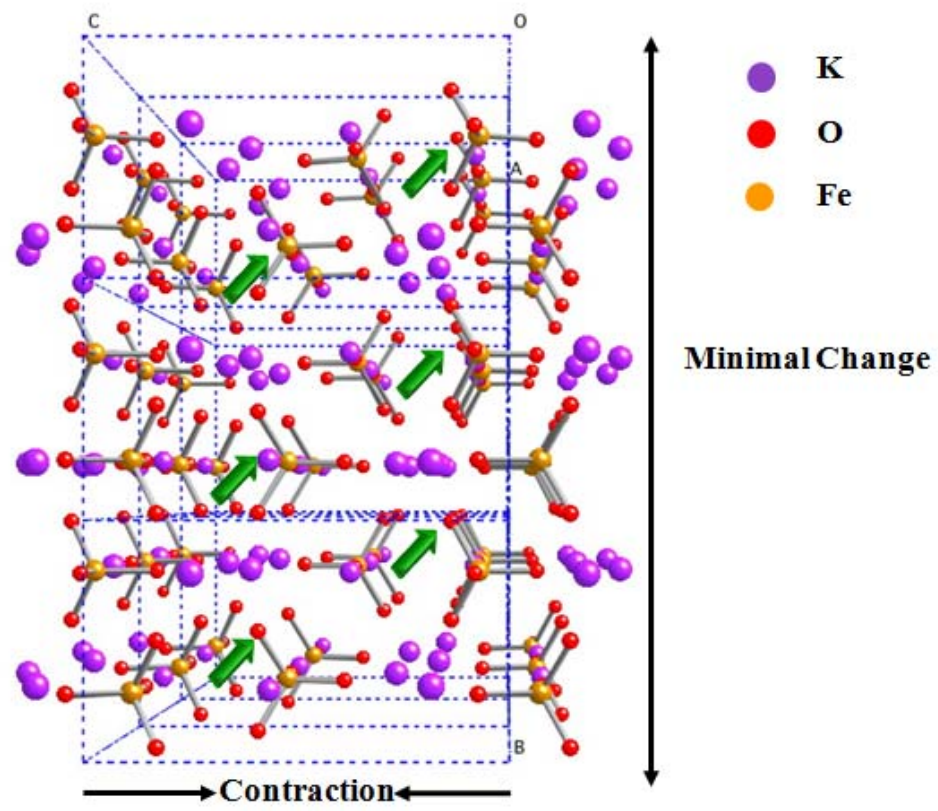

a) 


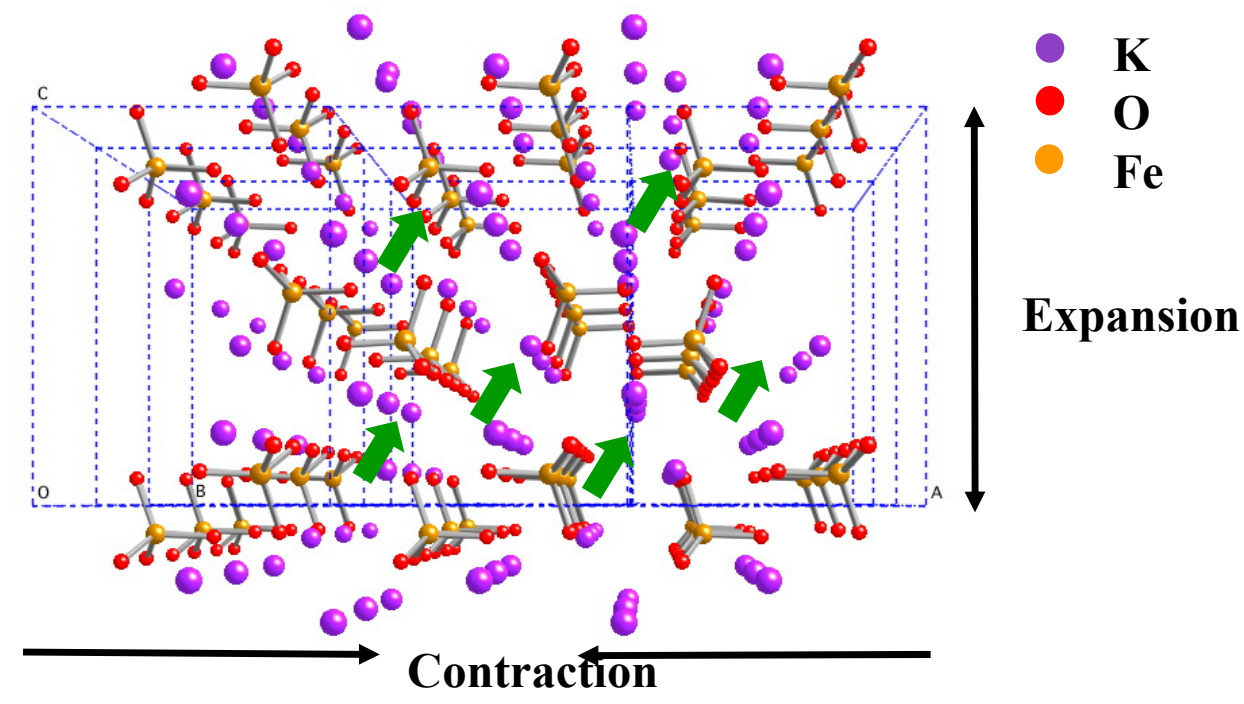

b)

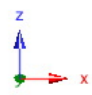

Figure 6.8. Unit cell of crystalline $\mathrm{K}_{2} \mathrm{FeO}_{4}$ along a) $\mathrm{x}$ and b) $\mathrm{y}$ axes. Green arrows show the direction of ion diffusion. Images Generated using CrystalMaker ${ }^{\circledR}$ : a crystal and molecular structures program for Mac and Windows. CrystalMaker Software Ltd, Oxford, England (www.crystalmaker.com) 
ease of diffusion of the intercalating ions are the two factors that determine the rate capabilities and reversibility of the cathode material under study. In the $\mathrm{BaFeO}_{4}$ lattice, every $\mathrm{Ba}$ atom is associated with two ferrate anions, compared to one $\mathrm{K}$ associated with each ferrate anion. The different interaction between cation and anion leads to different lattice parameters in these two compounds: $\mathrm{BaFeO}_{4}$ has an expanded lattice compared to $\mathrm{K}_{2} \mathrm{FeO}_{4}$. Table 6.1 shows XRD data previously obtained ${ }^{18,19}$, for both $\mathrm{K}_{2} \mathrm{FeO}_{4}$ and $\mathrm{BaFeO}_{4}$. The unit cell of $\mathrm{BaFeO}_{4}$ is expanded in the ' $\mathrm{a}$ ' and ' $b$ ' directions compared to that of $\mathrm{K}_{2} \mathrm{FeO}_{4}\left(9.13\right.$ and $5.85 \mathrm{~A}$ for $\mathrm{BaFeO}_{4}$ and 7.70 and $5.46 \mathrm{~A}$ for $\left.\mathrm{K}_{2} \mathrm{FeO}_{4}\right)$. In contrast, the unit cell in the 'c' direction is contracted in $\mathrm{BaFeO}_{4}(7.3232 \mathrm{~A})$ compared to that of $\mathrm{K}_{2} \mathrm{FeO}_{4}(10.3506 \mathrm{~A})$. Hence, the channels along the ' $\mathrm{a}$ ' and ' $\mathrm{b}$ ' axes are enlarged and more symmetrical in $\mathrm{BaFeO}_{4}$. The expanded lattice and more symmetrical diffusion channels facilitate the diffusivity of the $\mathrm{H}^{+}$along the channels enabling higher current densities and capacity. The expanded lattice of $\mathrm{BaFeO}_{4}$, and the stronger anioncation interaction apparently also causes less variation in the microstructure of $\mathrm{BaFeO}_{4}$ and less separation into $\mathrm{BaOH}_{2}$ and $\gamma-\mathrm{Fe}_{2} \mathrm{O}_{3}$ after discharge.

The Fourier transform XAS data shown in Figure 6.9 confirm that both the Fe-O and Fe-Fe bond lengths are increased in $\mathrm{BaFeO}_{4}$ compared to $\mathrm{K}_{2} \mathrm{FeO}_{4}$. Further, the widening of the Fe-O peak around $1.6 \AA$ indicates a larger variation in the $\mathrm{Fe}-\mathrm{O}$ bond lengths in $\mathrm{BaFeO}_{4}$, this is mostly likely caused by a larger interaction between the ferrate anion and the $\mathrm{Ba}$ cation. Clearly, $\mathrm{BaFeO}_{4}$ undergoes a smaller lattice expansion as a result of the 


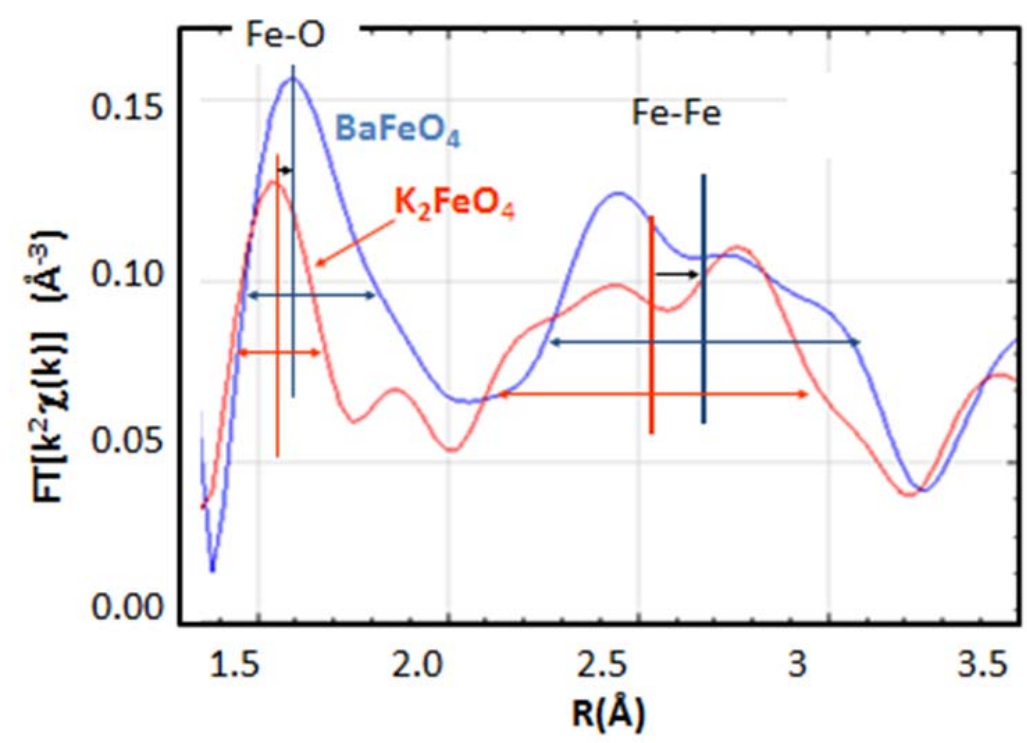

Figure 6.9. $\mathrm{K}_{2} \mathrm{FeO}_{4}$ and $\mathrm{BaFeO}_{4}$ Fourier transform prior to discharge; $\mathrm{BaFeO}_{4}$ shows increased $\mathrm{Fe}-\mathrm{O}$ and $\mathrm{Fe}-\mathrm{Fe}$ bond lengths and large $\mathrm{Fe}-\mathrm{O}$ peak width. 
intercalation process. The smaller lattice expansion, combined with more symmetrical intercalation channels, increases the reversibility of the intercalation process in $\mathrm{BaFeO}_{4}$. The larger ferrate-Ba cation interaction may also make it more difficult for the $\mathrm{BaOH}_{2}$ hydroxide and $\gamma-\mathrm{Fe}_{2} \mathrm{O}_{3}$ segregation to occur in the discharge product.

A comparison of the XRD data in Table 6.1 and electrochemical data previously obtained from constant load discharges of other $\mathrm{M}_{\mathrm{n}} \mathrm{FeO}_{4}$ salts as cathodes in alkaline coin cells given in Figure 6.10, confirms that an initial expanded lattice leads to a higher charge transfer rate (and as a result higher reversibility) in the cathode. $\mathrm{SrFeO}_{4}, \mathrm{Cs}_{2} \mathrm{FeO}_{4}$ and $\mathrm{BaFeO}_{4}$ all discharge to more than 80 percent of the $3 \mathrm{e}^{-}$transfer columbic efficiency under a constant load of 1000 ohms, whereas $\mathrm{K}_{2} \mathrm{FeO}_{4}$ only discharges up to $70 \%$. The XRD data in Table 6.1 shows that the unit cell in the 'a' direction for both $\mathrm{Cs}_{2} \mathrm{FeO}_{4}$ and $\mathrm{SrFeO}_{4}$ is larger than that of $\mathrm{K}_{2} \mathrm{FeO}_{4}\left(8.3998 \mathrm{~A}^{\circ}\right.$ for $\mathrm{Cs}_{2} \mathrm{FeO}_{4}$ and $9.1826 \mathrm{~A}^{\circ}$ for $\left.\mathrm{SrFeO}_{4}\right)$ and ' $b$ ' is increased for $\mathrm{CsFeO}_{4}\left(6.2737 \mathrm{~A}^{\mathrm{o}}\right)$. This correlation between XRD data and electrochemical data confirms the importance of an initial expanded lattice for increased discharge efficiency, charge transfer rate and reversibility. 


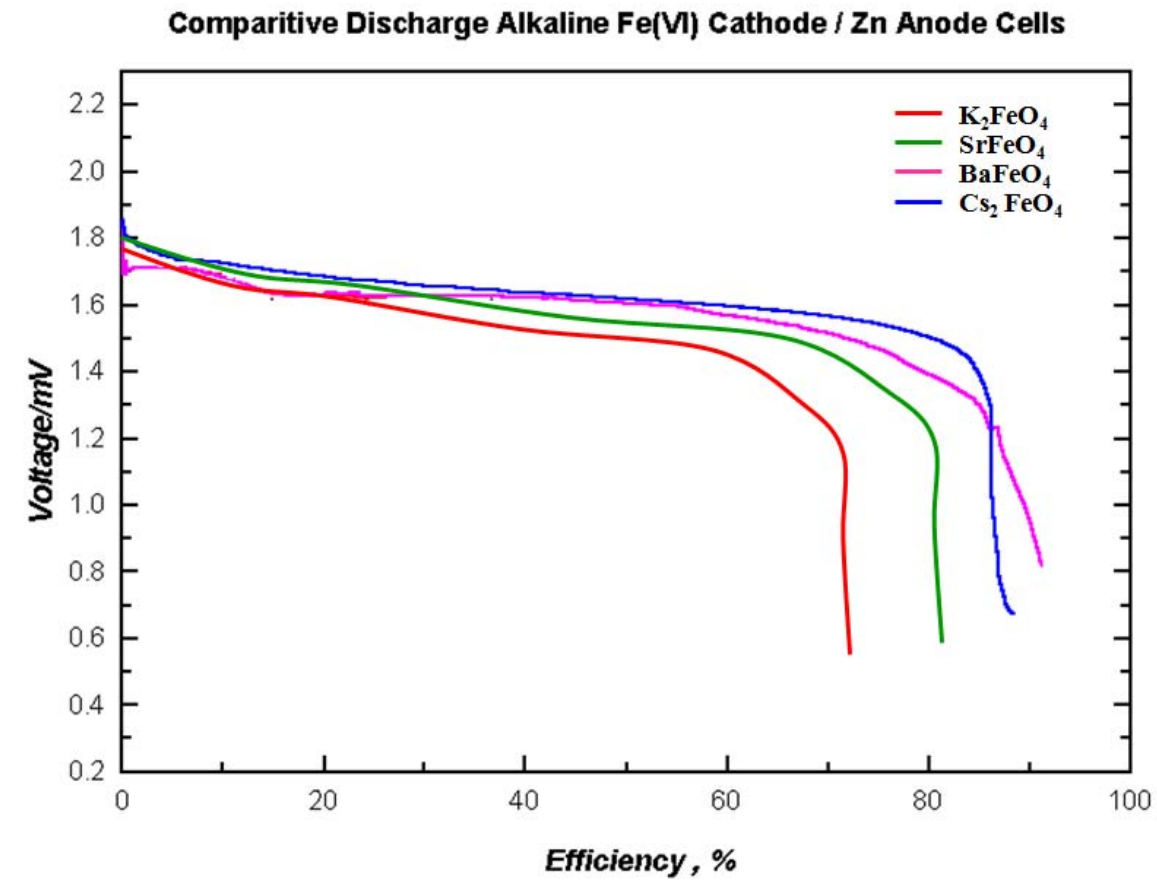

Figure 6.10. Comparative discharges of various $\mathrm{Fe}(\mathrm{VI})$ salt cathodes versus $\mathrm{Zn}$ anodes. $1 \mathrm{~cm}$ diameter coin cells were utilized, $300 \mathrm{mg}$ dry cathodes was mixed with $10 \mathrm{wt} . \%$ of $1 \mu \mathrm{m}$ graphite, electrolyte was saturated $\mathrm{KOH}$ and all cells were discharged with constant load of $1000 \mathrm{ohms}$. 


\begin{tabular}{|l|l|l|l|l|}
\hline Compound & Space Group & a, $A^{\circ}$ & b, $A^{\circ}$ & c, $A^{\circ}$ \\
\hline $\mathrm{K}_{2} \mathrm{FeO}_{4}$ & Pnma & $7.7010 \pm 0.0070$ & $5.8520 \pm 0.0064$ & $10.3506 \pm 0.0136$ \\
\hline $\mathrm{BaFeO}_{4}$ & Pnma & $9.1260 \pm 0.0110$ & $5.4563 \pm 0.0067$ & $7.3232 \pm 0.0083$ \\
\hline $\mathrm{Cs}_{2} \mathrm{FeO}_{4}$ & Pnma & $8.3998 \pm 0.0166$ & $6.2737 \pm 0.0105$ & $11.064 \pm 0.0230$ \\
\hline $\mathrm{SrFeO}_{4}$ & Pnma & $9.1826 \pm 0.0580$ & $5.3980 \pm 0.0263$ & $7.2256 \pm 0.0386$ \\
\hline
\end{tabular}

Table 6.1. $\mathrm{XRD} \mathrm{K}_{2} \mathrm{FeO}_{4}$ and $\mathrm{BaFeO}_{4}$ crystal data from Ref. 18 and $19 .{ }^{18,19}$ 


\subsection{Summary and Conclusions}

X-ray Absorption spectroscopy provides a valuable, highly specific technique to study materials used as novel electrodes in high capacity batteries. The $\Delta \mu$ technique allows one to follow the Fe oxidation state (hence capacity) and average local symmetry about the Fe atom and the EXAFS can be used to study the structural rearrangements and lattice variations of the electrode at any charge state. Ex-situ XAS measurements conducted on nanometer and micron- sized $\mathrm{K}_{2} \mathrm{FeO}_{4}$ and comparison to $\mathrm{BaFeO}_{4}$ at various

states of charge reveal the significance of minimizing the structural variations of the salt lattice and its' effects on the increased reversibility of the cathode. Micron size $\mathrm{BaFeO}_{4}$ particles hold their initial super iron salt structure to about $50 \%$ of the 3 -electron discharge similar to the behavior observed for the micron- $\mathrm{K}_{2} \mathrm{FeO}_{4}$ particles, but the discharge product remains more conductive enabling further discharge. Relieving the stress induced in the electrode's structure, caused by intercalation processes during charge/discharge cycles, is critical for increasing the cathode's charge transfer rate and electrochemical reversibility. Reduction of this intercalation induced stress is achieved either by accommodation of the lattice expansion and increase of the surface interface area (such as that enabled by decrease in particle size to the nano domain) or by minimization of the lattice expansion through the use of an initially enlarged lattice (such as $\mathrm{BaFeO}_{4}, \mathrm{SrFeO}_{4}$, etc.) and keeping the discharge product more uniform and less separated into hydroxide and iron oxide regions. 


\subsection{References}

(1) Gao, X.P.; Yang, H.X. Multi-electron reaction materials for high energy density batteries. Energy \& Environmental Science 2010, 3, 174-189.

(2) Licht S. Super-Iron Batteries. Garche, J.; Dyer, C.; Moseley, P.; Ogumi, Z.; David Rand D.; Scrosati, B. Encyclopedia of Electrochemical Power Sources 4; Amsterdam: Elsevier; 2009, 262-284.

(3) Licht, S. A High Capacity Li-Ion Cathode: The Fe(III/VI) Super-Iron Cathode. Energies 2010, 3, 960-972.

(4) Licht, S.; Wang, Y.; Gourdin, G. Enhancement of Reversible Nonaqueous Fe(III/VI) Cathodic Charge Transfer. J. Phys. Chem. C 2009, 113, 9884-9891.

(5) Licht, S.; De Alwis, C. Conductive-Matrix-Mediated Alkaline Fe(III/VI) Charge Transfer: Three-Electron Storage. J. Phys. Chem. B 2006, 110, 12394-12403.

(6) Farmand M.; Jiang D.; Wang B.; Ghosh, S.; Ramaker, D.E.; Licht, S. Super-iron nanoparticles with facile cathodic charge transfer. Electrochem. Comm. 2011, 13, 909-912.

(7) Licht, S.; Wang; B.; Ghosh, S. Energetic Iron(VI) Chemistry: The Super Iron Battery. Science 1999, 285, 1039-1042.

(8) Ghosh, S.; Wen, W.; Urian, R.C.; Heath, C.; Sirinvasmurthi, V.; Reiff, W.M.; Mukerjee, S.; Naschitz, V.; Licth, S. Reversible Behavior of $\mathrm{K}_{2} \mathrm{Fe}(\mathrm{VI}) \mathrm{O}_{4}$ in Aqueous Media. Electrochem. Solid-State Lett. 2003, 6, A260-a264.

(9) Licht, S.; Naschitz, V.; Halperin, L.; Halperin, N.; Lin, L.; Chen, J.; Ghosh, S.; Liu, B. Analysis of ferrate(VI) compounds and super-iron Fe(VI) battery cathodes: FTIR, ICP, titrimetric, XRD, UV/VIS, and electrochemical characterization. J. Power. Sources 2001, 101, 167-176.

(10) Ayersa, K.E.; White N.C. Characterization of Iron(VI) Compounds and Their Discharge Products in Strongly Alkaline Electrolyte. J. Electrochem. Soc. 2005, 152, A467-A473.

(11) Wang, Y.L.; Ye. S.H.; Wang, Y.Y.; Cao, J.S.; Wu, F. Structural and electrochemical properties of a $\mathrm{K} 2 \mathrm{FeO} 4$ cathode for rechargeable Li ion batteries. Electrochim. Acta 2009, 16, 4131-4135. 
(12) Whittingham, M. S.; Lithium Batteries and Cathode Materials. Chem. Rev. (Washington, D.C., U.S.) 2004, 104, 4271-4301.

(13) Fergus, J. W.; Recent developments in cathode materials for lithium ion batteries. $J$. Power Sources 2010, 195, 939-954.

(14) Bruce, P.G.; Scrosati, B.; Tarascon, J.M. Nanomaterials for Rechargeable Lithium Batteries. Angew Chemie Internat. Ed. 2008, 47, 2930-2946.

(15) Licht, S.; Yu, X. Recent advances in synthesis and analysis of Fe(VI) cathodes: solution phase and solid-state $\mathrm{Fe}(\mathrm{VI})$ syntheses, reversible thin-film $\mathrm{Fe}(\mathrm{VI})$ synthesis, coating-stabilized Fe(VI) synthesis, and Fe(VI) analytical methodologies. J. Solid State Electrochem., 2008, 12, 1523-1540.

(16) Licht, S.; Naschitz, V.; Liu, B.; Ghosh, S.; Halperin, N.; Halperin, L.; Rozen, D. Chemical synthesis of battery grade super-iron barrium and pottasium $\mathrm{Fe}(\mathrm{VI})$ ferrate compounds. Journal of Power Sources 2001, 99, 7-14.

(17) Licht, S.; Tel-Vered R.; Halperin L. Direct electrochemical preparation of solid $\mathrm{Fe}(\mathrm{VI})$ ferrate, and super-iron battery compounds. Electrochem. Comm. 2002, 4, 933-937.

(18) Herber, R.H.; Johnson D. Lattice Dynamics and Hyperfine Interactions in M2Fe04 $\left(\mathrm{M}=\mathrm{K}^{+}, \mathrm{Rb}^{+}, \mathrm{Cs}^{+}\right)$and $\mathrm{M}^{\prime} \mathrm{Fe} 04\left(\mathrm{M}^{\prime}=\mathrm{Sr}^{2+}, \mathrm{Ba}^{2+}\right)$. Inorg. Chem. 1979, 18, 27862790 .

(19) Audette, R.J.; Quail, J.W. Crystal Structures of M,FeO, (M = K, Rb, Cs), J. Solid State Chem. 1973, 8, 39-43.

(20) Newville, M. IFEFFIT: Interactive XAFS analysis and FEFF fitting. J. Synchrotron Radiat. 2001, 8, 322-324.

(21) Newville, M.; Livina, P.; Yacoby, Y.; Stern, E.A.; Rehr, J. Near-edge X-rayabsorption fine structure of $\mathrm{Pb}$ : A comparison of theory and experiment. J. Phys. Rev. B 1993, 47, 14126-14131.

(22) Shulman, R.G.; Yafet, Y.; Eisenberger, P.; Blumberg, W.E. Observations and interpretation of x-ray absorption edges in iron compounds and proteins. Proc. Natl. Acad. Sci. 1976, 73, 1384-1388. 
(23) Bruce, P.G.; Jiao, F. Mesoporous Crystalline $\beta-\mathrm{MnO}_{2}-$ a Reversible Positive Electrode for Rechargeable Lithium Batteries. Adv. Mater (Weinheim, Ger.) 2007, 19, 657-660.

(24) Bruce, P. G. Energy storage beyond the horizon: Rechargeable lithium batteries. Solid State Ionics 2008, 179, 752-760.

(25) Meethong, N.; Huang, H.Y.S.; Carter, W.S.; Chiang, Y.M.; Size-Dependent Lithium Miscibility Gap in Nanoscale $\mathrm{Li}_{1}-\boldsymbol{x} \mathrm{FePO}_{4}$. Electrochem. Solid-State Lett. 2007, 10, A134.

(26) Wanga, Y.L.; Yea, S.H.; Wanga, Y.Y.; Caoa, J.S.; Wu, F.B. Structural and electrochemical properties of a $\mathrm{K}_{2} \mathrm{FeO}_{4}$ cathode for rechargeable $\mathrm{Li}$ ion batteries. Electrochim. Acta 2009, 54, 4131-4135.

(28) Singh, G.K.; Ceder, G.; Bazant, M.Z. Intercalation dynamics in rechargeable battery materials: General theory and phase-transformation waves in $\mathrm{LiFePO}_{4}$. Electrochim. Acta 2008, 53, 7599-7613

(29) Bai, P.; Cogswell, D.A.; Bazant, M.Z. Suppression of Phase Separation in $\mathrm{LiFePO}_{4}$ nanoparticels during battery discharge. Nano Lett. 2011, 11, 4890-4896.

(30) Roscher, M.A.; Vetter, J.; Sauer, D.U. Characterization of charge and discharge behavior of lithium ion batteries with olivine based cathode active material. J. Power Sources 2009, 191, 582-590. 


\section{Chapter 7: Conclusions}

In this dissertation, electrochemical processes in fuel cells and batteries were followed by in-situ and ex-situ X-ray absorption spectroscopy (XAS) experiments. All of the XAS studies were complemented with electrochemical (rotating disk electrode, cyclic voltammetry, charge capacity), other spectroscopic (FTIR and XRD) and imaging measurements (SEM, HRTEM); the complementary data obtained for the most part by our collaborators on these projects. The $\Delta \mu$ XANES data analysis technique, previously developed and applied to heterogeneous catalysts and fuel cell electrocatalysts by the GWU group, was extended in this work to other applications, and more importantly for the first time applied to battery relevant materials, where bulk properties such as the oxidation state and local geometry of a cathode are followed. In this concluding chapter, a summary of the results obtained is given, and possible future directions are discussed.

\subsection{Adsorption of bromide and iodide on platinum}

Chapter 3 reported a comparative study of poisoning effects of bromide and iodide on the oxygen reduction reaction (ORR) activity of polycrystalline $\mathrm{Pt}$ in acidic medium (1M $\mathrm{HClO}_{4}$ ). This project was a follow up to a project previously conducted and published ${ }^{1}$ on the poisoning effects of chloride on Pt. In-situ XAS experiments were complemented with electrochemical characterization techniques (RDE and $\mathrm{CV}$ ) and conducted mainly at two different concentrations, $1 \mathrm{mM}$ and $10 \mathrm{mM}$ halide in the electrolyte. Rotating disk electrode (RDE) experiments on $\mathrm{Br}^{-}$showed that even at the lower concentration $(1 \mathrm{mM})$ the ORR overpotential increases by $200 \mathrm{mV}$. CV experiments showed that both bromide and iodide 
largely affected all three potential regions, namely the $\mathrm{H}_{\text {upd, }}$, double-layer and Pt-O formation regions. Iodide suppressed and affected the CVs more than bromide, pointing to even more severe poisoning effects with iodide.

The work in Chapter 1 provided not only increased insight into the comparative poisoning effects of halides on the ORR in a fuel cell, but also new insights into the nature of the $\Delta \mu$ magnitude itself. In the case of strongly bonded adsorbates (e.g., $\mathrm{O}, \mathrm{OH}, \mathrm{CO}$ and $\mathrm{H}$ ) on a Pt electrode in a fuel cell, such as that studied in all previous applications of the $\Delta \mu$ technique, the $\Delta \mu$ magnitude provided a reasonable estimate of the relative adsorbate coverage as a function of potential. However, in the case of the large, highly polarizable and weakly bonded anions, such as the halides in this work, this is not the case, especially at lower concentrations of the adsorbate. In this special case, the $\Delta \mu$ magnitudes were not directly proportional to $\mathrm{X}^{-}$coverage, but decreased as the Pt-X bond changed covalent character with potential. In this way, the $\Delta \mu$ provided some insight into the varying nature of the Pt-X bond; however, at the expense of the more appealing linear behavior seen previously. Although some degree of non-linear behavior is nearly a

lways to be expected, this latest work suggest that when quantitative adsorbate coverages are needed, one must be careful when making this linear $\Delta \mu$ magnitude vs. coverage assumption

\subsection{Space-resolved, in Operando XAS investigations in a DMFC}

In chapter 4 of this dissertation, in-operando XAS experiments were conducted to follow the spatial distribution of different adsorbates on both the anode and cathode in a direct methanol fuel cell (DMFC). These were the first space resolved $\Delta \mu$ studies 
performed on working fuel cells. Specifically, we were interested in the spatial correlation of adsorbates (such as $\mathrm{OH}, \mathrm{H}, \mathrm{O}$, and $\mathrm{CO}$ ) on the anode and immediately across the proton exchange membrane (PEM) on the cathode side. These studies were conducted with two different oxygen flow rates: under high oxygen supply $(150 \mathrm{~mL} / \mathrm{min})$ and under oxygen starvation conditions $(20 \mathrm{~mL} / \mathrm{min})$. The motivation for looking at two different oxygen flow rates was to observe the different operational modes possible under these conditions; i. e., it has been previously noted that under low oxygen flow rates the cathode can start to function at two different operational modes (galvanic and electrolytic) simultaneously. ${ }^{3-6}$ To investigate the adsorbate spatial distributions, a specially designed in-situ cell was utilized, which has three different X-ray windows at different locations relative to the inlets and outlets of the fuel (methanol) and oxidizer $\left(\mathrm{O}_{2}\right)$ feeding the cell. With this cell both fluorescence and transmission data could be collected at both the cathodic and anodic sides. The $\Delta \mu$-XANES technique was utilized to analyze the data gathered at both flow rates and at both the cathode and anode.

The results revealed a very strong "cross-talk" between the anode and cathode, i.e. a strong correlation between the adsorbates on the anode and cathode side. The "cross-talk" was previously detected through neutron imaging studies, but this is an indirect approach as it does not specifically follow the spatial adsorbate coverage and distribution. At higher oxygen flow rates, it was found that it is the anode that dictates the $\mathrm{OH}$ coverage on the cathode, because every step in the reduction of $\mathrm{O}_{2}$ to adsorbed $\mathrm{OH} / \mathrm{Pt}$ requires a proton, and these $\mathrm{H}^{+}$come through the PEM after they are produced at the anode. At lower oxygen flow rate, this "cross-talk" effect is reversed with the cathode dictating the methanol 
oxidation rate on the anode because of slow $\mathrm{H}^{+}$removal and methanol cross-over. These results can be simply summarized by indicating that the local adsorbate coverage on the anode or cathode is determined by the electrode with the slowest reaction rate.

Over the years, the exact mechanism for the oxygen reduction reaction has been controversial, and various rate determining steps have been proposed. However, in all of these proposed mechanisms, the presence of $\mathrm{H}^{+}$is vital in every step. Our results directly highlight the importance of the local $\mathrm{H}^{+}$concentration at the cathode. Under $\mathrm{O}_{2}$ starvation conditions, the results reveal the nature of the adsorbates on both the anode and cathode, when the DMFC is operating "bifunctionally", i.e. in galvanic mode at low cell potential and in electrolytic mode at high cell potential. Our results under $\mathrm{O}_{2}$ starvation correlate with results previously obtained by Sauer et. al. through current density measurements. ${ }^{6}$

\subsection{Super-iron nanoparticles with facile cathodic charge transfer}

In Chapter 5, the particle size effect on the electrochemical performance of super-iron salts as high capacity cathodes was studied utilizing XAS. The super-iron salts are a class

of novel multi-electron cathodes introduces by Light et al. ${ }^{8}$ These hexavalent salts release 3 e- upon reduction and can offer intrinsic capacities as high as $601 \mathrm{mAh} / \mathrm{g}$. However, their discharge product suffers from poor conductivity and low reversibility. The exact nature of this ferric discharge product has not been fully identified. Here, a new low heat, gentle and mechano-synthesized pathway was employed to produce nano-sized $\mathrm{K}_{2} \mathrm{FeO}_{4}$ particles. SEM results showed that the particle size was reduced from around 30 microns to about 80100 nanometers by this ball mill procedure. Nanoparticles, when used as cathodes in 
alkaline batteries, improve the discharge Columbic efficiency to $\sim 80 \%$, compared to the previously observed $60 \%$ for micron-sized particles.

Ex-situ XAS measurements were conducted on both the micron and nano-sized cathodic composites discharged to various depths under constant load. The final discharge product was identified as primarily $\gamma-\mathrm{Fe}_{2} \mathrm{O}_{3}$. The $\Delta \mu$ amplitude of the pre-edge peak (1s to 3d transition) was utilized to follow the $\mathrm{Fe}$ oxidation state changes while linear combination fitting analysis of the EXAFS region was utilized to follow the structure. Here the standard components of the fit were the undischarged $\mathrm{K}_{2} \mathrm{FeO}_{4}$ and $\gamma-\mathrm{Fe}_{2} \mathrm{O}_{3}$. It was illustrated that nano-particles do not show any structural change up to nearly $55 \%$ of the discharge, while structural changes for micron-sized particles start as low as $15 \%$ of the discharge.

These results provide insight into the importance of inhibiting structural variations in the electrode during the charge/discharge cycle in order to improve the reversibility of the cathode. In this battery material, the $\Delta \mu$ analysis technique was not used to follow the adsorbate coverage and surface reactions, but instead as a tool to follow the oxidation state, and indirectly the capacity of the electrode as the electrochemical reaction proceeds.

\subsection{Studying the Reversibility of Multi-electron Charge Transfer in Fe(VI) Cathodes}

Chapter 6 presents a more in-depth look at a number of super-iron salts and their multi-electron charge transfer reversibility. Ex-situ measurements were conducted on nano$\mathrm{K}_{2} \mathrm{FeO}_{4}$, micron-sized $\mathrm{K}_{2} \mathrm{FeO}_{4}$ and micron-sized $\mathrm{BaFeO}_{4}$. Here, $\mathrm{BaFeO}_{4}$ was selected because of its previously observed higher charge transfer rate and experimental capacity. However, $\mathrm{BaFeO}_{4}$ has a lower thermal stability and theoretical capacity compared to that of 
$\mathrm{K}_{2} \mathrm{FeO}_{4} \cdot{ }^{9}$ Again, alkaline batteries were constructed with super-iron salt as cathode and discharged to various depths and studied through XAS experiments. The $\Delta \mu$ analysis was conducted both on the Fe pre-edge which originates from 1s to $3 \mathrm{~d}$ transitions and on the $\mathrm{Fe}$ K-edge at $7112.0 \mathrm{eV}$, which reflects the $1 \mathrm{~s}$ to $\mathrm{np}$ transitions. In this chapter the K-edge $\Delta \mu$ amplitudes serve as a tool to follow the Fe oxidation state change, while the pre-edge $\Delta \mu$ amplitudes reflect the local geometry change of the hexavalent Fe salt from octahedral to tetrahedral. Thus Chapter 8 significantly extended the work in Chapter 7, not only by looking at a wider array of materials, but now distinguishing between the Fe oxidation state and distortions of the local Fe geometry. Linear combination fits were again conducted on the EXAFS region, with the undischarged salt and final discharge product $\left(\gamma-\mathrm{Fe}_{2} \mathrm{O}_{3}\right)$ as components, to follow the structural changes as the discharge proceeds.

The micron sized $\mathrm{BaFeO}_{4}$ particles held their initial tetrahedral structures stable up to approximately $50 \%$ of the 3 -electron discharge process, and the higher discharge product conductivity leads to a higher experimental capacity. A similar behavior is observed with the nano-sized $\mathrm{K}_{2} \mathrm{FeO}_{4}$, holding the structure stable deep into the discharge. Our XAS results show that the mechanism by which the $\mathrm{BaFeO}_{4}$ and nano- $\mathrm{K}_{2} \mathrm{FeO}_{4}$ materials hold their structures stable are quite different. During the discharge process in these alkaline batteries, $\mathrm{H}_{2} \mathrm{O}$ intercalates into the cathode lattice through the electrolyte and membrane separator, while electrons travel from the anode to the cathode through the external circuit. Nano-particles are able to relieve the stress induced by the oxidation through intercalation of water. This is possible because of the shorter diffusion lengths and the increased electrode/electrolyte surface in the nanoparticle. This water intercalation enables the Fe to 
maintain its original local geometry, and the lattice expansion caused by this intercalation is better accommodated in the micron-sized particles. In the case of $\mathrm{BaFeO}_{4}$, previous $\mathrm{XRD}$ data ${ }^{10}$ illustrated that the one dimensional intercalation channels are expanded and more symmetrical compared to that of the micron sized $\mathrm{K}_{2} \mathrm{FeO}_{4}$. This is supported by our XAS data, where the Fourier transform EXAFS data shows increased Fe-O and Fe-Fe bond lengths and widened peaks in $\mathrm{BaFeO}_{4}$ compared to $\mathrm{K}_{2} \mathrm{FeO}_{4}$. This shows that $\mathrm{BaFeO}_{4}$ starts with an already expanded lattice and intercalation of $\mathrm{H}^{+}$to the cathode is facilitated. This is also the reason behind the higher charge transfer rates in $\mathrm{BaFeO}_{4}$. Further, stronger cationanion interactions in $\mathrm{BaFeO}_{4}$ also delays the phase separation of the discharge products, $\mathrm{Ba}(\mathrm{OH})_{2}$ and $\gamma-\mathrm{Fe}_{2} \mathrm{O}_{3}$. All of these factors contribute to higher structural stability observed in $\mathrm{BaFeO}_{4}$, increasing its reversibility for more charge/discharge cycles. Hence, to increase the reversibility of super-iron salts either the particle size should be reduced to the nano domain or a salt with higher anion-cation interaction and initial expanded lattice should be utilized.

Chapters 5 and 6 both point to the importance of spectrochemical/electrochemical characterization techniques for intelligent design of novel materials for applications in energy storage devices. The combination of the $\Delta \mu$-XANES analysis technique along with electrochemical and other characterization techniques such as FTIR and SEM can provide valuable insight into the electrochemical reaction mechanisms, and provide feedback for optimized materials design. 


\subsection{Future Directions}

Fuel cell technology still has many challenges to overcome before full commercialization as power sources, either in mobile or stationary applications, can be realized. Recent advances in XAS instrumentation enables this characterization technique to be utilized in new and previously un-explored capacities. For instance, beamlines, such as those at the Paul Scherrer Institute in Switzerland, now provide fast scanning capabilities with milli-second time resolution, small spot size on the sample $\left(100 * 100 \mu \mathrm{m}^{2}\right)$, and good energy resolutions $\left(0.5 * 10^{-4}\right.$ for $\left.\mathrm{Si}(311)\right)$ allow for better spatial resolution and even time resolved studies. ${ }^{11}$ These new x-ray sources can be utilized to expand our $\Delta \mu$ spatial studies into virtually an "imaging" technique, where instead of three limited windows such as used in this work, the entire electrode can be "scanned" at hundreds of spots over the entire electrode. "Imaging" electrodes at both sides (anode and cathode) can provide insight into various processes on the surface of the electrode such as buildup and evolution of water on the cathode, flow of methanol through the membrane, etc., and correlate them with current density studies.

These new capabilities can also provide new avenues for poisoning studies. Timeresolved measurements could be carried out to study the poisoning and reaction mechanisms at both the cathode and anode, such as results recently published by Melke et. al. ${ }^{12}$ utilizing time resolved $\Delta \mu$-XANES data to quantitatively estimate the adsorbate coverages on $\mathrm{Pt} / \mathrm{C}$ during the ethanol oxidation reaction.

The next step in improving the reversibility, and therefore potential for application of super-iron salts as high capacity cathodes for energy storage, is to study them in non- 
aqueous batteries. As mentioned in Chapter 1, it has previously been illustrated that thin films deposited on platinized electrodes increase the reversibility of these salts making a high number of charge/discharge cycles possible. Here, we showed that utilizing nanosized particles can also increase the reversibility of these salts. In the future, nano superiron salts should be studied when coupled with high capacity anodes in a non-aqueous medium. These studies can easily be conducted in-situ, as super-iron salts have lower solubility in commonly used non-aqueous solvents compared to alkaline electrolytes. ${ }^{13} \mathrm{We}$ have previously attempted to conduct in-situ studies on rechargeable alkaline super-iron batteries using both deposited thin films on platinized platinum and nano-particles. However, the increased dissolution of these salts when exposed to alkaline electrolyte, and other decay processes when under beam conditions, prevented us from acquiring data with an acceptable signal to noise ratio. This problem could easily be alleviated when using non-aqueous systems due to the lower solubility in such solvents.

Novel electrochemical synthesis routes could also be explored to produce nano-sized lithiated ferrate salts, which could potentially increase the cathode capacity as high as 606 $\mathrm{mAh} / \mathrm{g}$. For instance, various nano-sized lithium iron salts could be utilized as precursors to electrochemically synthesize nano-sized lithiated super iron cathodes. XAS studies will be crucial in studying the structural stability and subsequently the reversibility of multielectron charge transfer in these novel cathode materials.

Another barrier to increasing the energy capacity of batteries is the anode capacity. So far, super iron salts have been studied as cathodes in alkaline batteries or in non-aqueous batteries versus a lithium or graphite anode. One of the frontiers for future energy storage 
devices is the development of novel anodic materials such as Sn based materials. For instance, active/inactive nano-composite alloys have been proposed as high capacity anodes that show highly rechargeable characteristics compared to traditional anodes used in Li ion batteries. These materials have a high rechargeability since their inert matrix buffers the volumetric expansions of the Sn-based active component. These materials show considerably higher capacities and reversibility compared to anodes currently utilized. For example, encapsulated Sn nanoparticles in multiwalled carbon nanotubes (CNTs) have shown first cycle insertion and de-insertion capacities of 2472 and $889 \mathrm{mAh} / \mathrm{g}$, respectively. These anodic composites were able to maintain reversible capacities in the $720-800 \mathrm{mAh} / \mathrm{g}$ range for the first 20 cycles. ${ }^{14}$ XAS studies can be utilized to study the applicability of these anodes versus lithiated nano super-iron salts and intercalation mechanisms in such systems. 


\subsection{References:}

(1) Arruda, T. A.; Shyam, B.; Ziegelbauer, J. M.; Mukerjee, S.; Ramaker, D. E. Investigation into the Competitive and Site-Specific Nature of Anion Adsorption on PtUsing In Situ X-ray Absorption Spectroscopy. J. Phys. Chem. C 2008, 112, 18087.

(2) Lucas, C. A.; Markovic, N. M.; Ross, P. N. Adsorption of halide anions at the $\operatorname{Pt}(111)$-solution interfacestudied by in situ surface x-ray scattering. Phys. Rev. $B$ 1997, 55, 7964.

(3) Kulikovsky, A. A.; Schmitz, H.; Wippermann, K.; Mergel, B.; Fricke, T.; Sanders, D. U. DMFC: Galvanic or electrolytic cell? Electrochem. Commun. 2006, 8,754 .

(4) Mueller, H.; Dohle, A. A.; Kulikovsky, A. A. Electrochemical Reactions in a DMFC under Open-Circuit Conditions. Electrochem. Solid State Lett. 2006, 9, L7.

(5) Schröder, A.; Wippermann, K.; Mergel, J.; Lehnert, W.; Stolten, D.; Sanders, T.; Baumhöfer, T.; Sauer, D. U.; Manke, I.; Kardjilov, N. Combined local current distribution measurements and high resolution neutron radiography of operating Direct Methanol Fuel Cells. Electrochem. Commun. 2009, 11, 1606.

(6) Kulikovsky, A. A.; Schmitz, H.; Wippermann, K.; Mergel, J.; Fricke, B.; Sanders, T.; Sauer, D. U. Bifunctional activation of a direct methanol fuel cell. $J$. Power Sources 2007, 173, 420.

(7) Ye, Q.; Zhao, H.; Yang, J.; Prabhuran, J. Electrochemical Reactions in a DMFC under Open-Circuit Conditions. J. Electrochem. Solid-State Lett. 2005, 8, A52.

(8) Licht, S.; Wang, B.; Ghosh, S. Energetic Iron(VI) Chemistry: The SuperIron Battery. Science 1999, 285, 1039.

(9) Licht, S.; Yu, X. Recent advances in synthesis and analysis of $\mathrm{Fe}(\mathrm{VI})$ cathodes: solution phase and solid-state $\mathrm{Fe}(\mathrm{VI})$ syntheses, reversible thin-film $\mathrm{Fe}(\mathrm{VI})$ synthesis, coating-stabilized $\mathrm{Fe}(\mathrm{VI})$ synthesis, and $\mathrm{Fe}(\mathrm{VI})$ analytical methodologies. J. Solid State Electrochem. 2008, 12, 1523.

(10) Wanga, Y. L.; Yea, S. H.; Wanga, Y. Y.; Caoa, J. S.; Wu, F. B. Structural and electrochemical properties of a $\mathrm{K}_{2} \mathrm{FeO}_{4}$ cathode for rechargeable $\mathrm{Li}$ ion batteries. Electrochim. Acta 2009, 54, 4131.

(11) http://www.psi.ch/sls/superxas/superxas. 
(12) Melke, J.; Schoekel, A.; Ditty Dixon, D.; Cremers, C.; Ramaker, D. E.; Roth, C. Ethanol Oxidation on Carbon-Supported Pt, PtRu, and PtSn Catalysts Studied by Operando X-ray Absorption Spectroscopy. J. Phys. Chem. C 2010, 114, 5914.

(13) Licht, S.; Wang, B. Nonaqueous Phase Fe(VI) Electrochemical Storage and Discharge ofSuper-Iron/Lithium Primary Batteries. Electrochem. Solid State Lett 2000, 3, 209.

(14) Zheng, J. W.; Nai, S. M. L.; Ng, M. F.; Wu, P.; Wei, J.; Gupta, M. DFT Study on Nano Structures of $\mathrm{Sn} / \mathrm{CNT}$ Complex for Potential Li-Ion Battery Application. J. Phys. Chem. C 2009, 113, 14015 